Campos hipoelíticos no plano 

SERVIÇO DE PÓS-GRADUAÇÃO DO ICMC-USP

Data de Depósito:

Assinatura:

\section{Campos hipoelíticos no plano}

\section{Camilo Campana}

Orientador: Prof. Dr. Adalberto Panobianco Bergamasco

Dissertação apresentada ao Instituto de Ciências Matemáticas e de Computação - ICMC-USP, como parte dos requisitos para obtenção do título de Mestre em Ciências - Matemática . VERSÃO REVISADA

\section{USP - São Carlos}

Abril de 2013 
Ficha catalográfica elaborada pela Biblioteca Prof. Achille Bassi e Seção Técnica de Informática, ICMC/USP, com os dados fornecidos pelo(a) autor(a)

\begin{tabular}{|c|c|}
\hline $\mathrm{C} 186 \mathrm{C}$ & $\begin{array}{l}\text { Campana, Camilo } \\
\text { Campos hipoelíticos no plano / Camilo Campana; } \\
\text { orientador Adalberto Panobianco Bergamasco. -- São } \\
\text { Carlos, } 2013 \text {. } \\
\quad 155 \text { p. }\end{array}$ \\
\hline & $\begin{array}{l}\text { Dissertação (Mestrado - Programa de Pós-Graduação } \\
\text { em Matemática) -- Instituto de Ciências Matemáticas } \\
\text { e de Computação, Universidade de São Paulo, } 2013 \text {. } \\
\text { 1. campos vetoriais complexos. } 2 . \\
\text { hipoeliticidade. 3. uniformização. } 4 . \\
\text { integrabilidade local. 5. resolubilidade local. I. } \\
\text { Panobianco Bergamasco, Adalberto, orient. II. } \\
\text { Título. }\end{array}$ \\
\hline
\end{tabular}


Aos meus pais, avós e bisavós. 



\section{Agradecimentos}

\section{À Deus.}

Ao programa de pós graduação do USP-ICMC (Instituto de Matemática Aplicada e Computação). Aos professores e funcionários que contribuíram para a confecção dessa dissertação.

Em especial ao meu orientador e amigo, Adalberto Panobianco Bergamasco, pela disponibilidade, grande dedicação e pelos conhecimentos transmitidos.

À minha família, especialmente, meus pais Lourdes e Judçon. Meus avós, Jovelina e Estevaldo e aos meus bisavós Maria e Américo. Também a minha prima, Ana Carolina Campana que me ajudou no início da graduação.

Aos meus amigos. Em especial para Alomir, Dione, Steve, Laís e Rodrigo. Também ao amigo Vinícius, pela ajuda no início do mestrado.

Aos professores da UFES, Alancardek Pereira Araujo, Ana Claudia Locatelli, José Armínio Ferreira, Júlia Shaetzle Wrobel, Magda Soares Xavier e Ricardo Soares Leite.

Aos professores do ensino médio que me direcionaram para esta caminhada, Antônio Marcos Maifredi e Muriel Belique Sangali. 
À todos os citados e as pessoas que não mencionei, mas que fizeram parte da minha trajetória, fica registrado o meu agradecimento.

À CAPES pelo apoio financeiro. 


\section{Resumo}

Seja $L$ um campo vetorial complexo não singular definido em um aberto do plano. Treves provou que se $L$ é localmente resolúvel então $L$ é localmente integrável. Para campos planares hipoelíticos, vale uma propriedade adicional, a saber, toda integral primeira (restrita a um aberto suficientemente pequeno) é uma aplicação injetiva (e aberta); isto, por sua vez, implica que toda solução da equação homogênea $L u=0$ é localmente da forma $u=h \circ Z$, com $h$ holomorfa, sendo $Z$ uma integral primeira do campo. O problema central de interesse desta dissertação é a questão global correspondente, ou seja, a existência de integrais primeiras globais injetoras e a representação de soluções globais por composições da integral primeira com uma função holomorfa. 
Palavras-chave: campos vetoriais complexos; hipoeliticidade; uniformização; integrabilidade local; integrabilidade global; resolubilidade local; condição (P). 


\section{Abstract}

Let $L$ be a nonsingular complex vector field defined on an open subset of the plane. Treves proved that if $L$ is locally solvable then $L$ is locally integrable. For hypoelliptic planar vector fields an additional property holds, namely, every first integral (restricted to a sufficiently small open set) is an injective (and open) mapping; this, on its turn, implies that each solution of the homogeneous equation $L u=0$ is locally of the form $u=h \circ Z$, where $h$ is holomorphic and $Z$ is a first integral of the vector field. The central problem of interest in this work is the corresponding global question, that is, the existence of global, injective first integrals and the representation of global solutions as compositions of the first integral with a holomorphic function.

Key words: complex vector fields; hypoellipticity; uniformization; local integrability; global integrability; local solvability; condition (P). 



\section{Sumário}

Introdução

1 Pré-requisitos 5

1.1 Notação . . . . . . . . . . . . . . . . . . . . . 5 5

1.2 Espaços Vetoriais Topológicos . . . . . . . . . . . . . . . . 8

1.2.1 A topologia de $C_{c}^{\infty}(\Omega)$ e o espaço $\mathscr{D}^{\prime}(\Omega) \ldots \ldots \ldots \ldots$

1.2.2 Mudança de Variáveis em Distribuições . . . . . . . . . . . . . . . 18

1.3 Operadores Diferenciais Parciais Lineares . . . . . . . . . . . . . . 20

1.4 Alguns resultados de Análise Complexa . . . . . . . . . . . . . . . 23

1.4.1 O Operador de Cauchy-Riemann . . . . . . . . . . . . 24

1.4.2 Alguns teoremas . . . . . . . . . . . . 26

1.4.3 Convergência de sequências de funções holomorfas . . . . . . . . . . 27

1.5 Campos Vetoriais Complexos . . . . . . . . . . . . . 28

1.5.1 Campos Vetoriais Complexos ................ 28

1.5.2 Formas Diferenciais . . . . . . . . . . . . . . . . . 34

1.5.3 Mudança de Variáveis em Campos . . . . . . . . . . . . . 37

2 Superfícies de Riemann $\quad 47$

2.1 Definição . . . . . . . . . . . . . . . . . . . . . 47

2.2 Uniformização . . . . . . . . . . . . . . . . . . . 53

2.2.1 O Teorema de Uniformização para Superfícies de Riemann Simplesmente Conexas ........................ 53 
2.2.2 Uniformização para Superfícies de Riemann não Simplesmente Co-

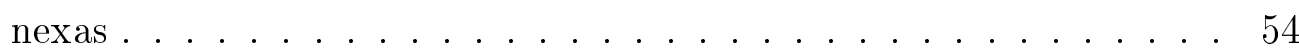

2.3 Alguns comentários . . . . . . . . . . . . . . . . . 55

3 Resolubilidade $\quad 57$

3.1 Resolubilidade Local . . . . . . . . . . . . . . . . . 57

3.2 A Condição $(\mathrm{P}) \ldots \ldots \ldots \ldots \ldots \ldots \ldots$

3.3 Resolubilidade no caso hipoelítico . . . . . . . . . . . . . . . 63

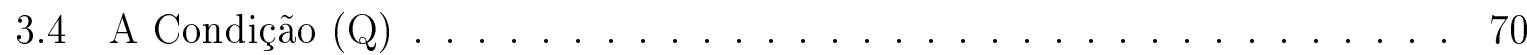

3.5 Integrabilidade Local no Plano . . . . . . . . . . . . . 76

4 Caso Elítico $\quad 79$

4.1 Introdução . . . . . . . . . . . . . . . . . . . 79

4.2 Eliticidade e Integrabilidade Local . . . . . . . . . . . . . . . . . 79

4.2.1 Redução de um Campo Elítico a um múltiplo do Operador de Cauchy-Riemann ................... 86

4.3 Estrutura Diferenciável Complexa induzida por um Campo Elítico . . . . . 89

4.4 Solução do Problema . . . . . . . . . . . . . . . . . . . 92

$\begin{array}{lll}5 & \text { Caso Hipoelítico } & 97\end{array}$

5.1 Introdução . . . . . . . . . . . . . . . . . 97

5.2 Hipoeliticidade e Integrabilidade Local . . . . . . . . . . . . . . . 97

5.3 Teorema de Aproximação e Estrutura das Soluções . . . . . . . . . . . . 113

5.3.1 Teorema de Aproximação de Baouendi-Treves . . . . . . . . . . . 113

5.3.2 Estrutura Local das Soluções . . . . . . . . . . . . . . . 130

5.4 Estrutura Diferenciável Complexa induzida por um Campo Hipoelítico . 136

5.5 Solução do Problema . . . . . . . . . . . . . . . . . . . . 139

A Solução da Equação de Beltrami 143

A.1 Espaços de Hölder . . . . . . . . . . . . . . . . . . . . . 143 
A.2 A Equação de Beltrami e o Método do Ponto Fixo . . . . . . . . . . . . . 144 


\section{Introdução}

Na década de 1960 o estudo de resolubilidade local de operadores diferenciais parciais foi alavancado pelos trabalhos publicados por Nirenberg e Treves. Em um desses trabalhos foi formulada a condição (P), a qual um bom tempo depois se mostrou necessária e suficiente para a resolubilidade local de um operador diferencial parcial linear de tipo principal (por exemplo, campos vetoriais complexos não singulares). Assim, surgiram novos problemas relacionados a campos vetoriais complexos; um deles consiste em decidir se um campo é localmente integrável,i.e., se para cada ponto $p$ do domínio de um campo $L$, existe uma solução $u$ de $L$ (i.e., uma distribuição definida numa vizinhança de $p$, tal que $L u=0$ nesta vizinhança), que seja $C^{\infty}$ e satisfaça $d u \neq 0$. A função $u$ é chamada de uma integral primeira do campo L. Como o próprio nome diz, a integrabilidade local é um conceito local. No entanto, surge a seguinte pergunta, de caráter global, sobre este problema: se L é campo vetorial complexo não singular definido em um aberto $U$ de $\mathbb{R}^{n}$, existe uma integral primeira global $Z$, i.e., existe $Z$ função $C^{\infty}$ definida em todo o aberto U tal que

$$
L Z=0, d Z \neq 0 \text { em } U \text { ? }
$$

Em [3] esta pergunta foi respondida em uma classe de campos, os campos hipoelíticos definidos em abertos simplesmente conexos do plano, com o adicional de que a solução obtida é injetora.

Um dos objetivos deste trabalho é estudar com detalhes a resposta dada em [3]. Mais especificamente, a questão central deste trabalho é mostrar que, quando $L$ é um campo 
vetorial complexo não singular hipoelítico definido em um aberto conexo $\Omega \subset \mathbb{R}^{2}$, o seguinte problema:

$$
L Z=0, d Z \neq 0 \text { e } Z \text { injetiva em } \Omega
$$

tem solução global quando o grupo fundamental do aberto $\Omega$ é trivial, i.e., $\Omega$ simplesmente conexo, e quando o grupo fundamental de $\Omega$ é $\mathbb{Z}$, ou seja, quando $\pi_{1}(\Omega)=\{0\}$ ou quando $\pi_{1}(\Omega)=\mathbb{Z}$.

Uma motivação para o método usado para obter a solução é inspirado no caso em que o campo $L$ é elítico. Um fato (que discutiremos no capítulo 4) sobre campos elíticos é que localmente existe uma mudança de coordenadas a qual grosso modo é uma solução de $L$ e transforma $L$ em um múltiplo do operador de Cauchy-Riemann. Todas as soluções de $L$ nessas novas coordenadas são funções holomorfas. Assim, $L$ induz uma estrutura diferencial complexa em $\Omega$ (capítulo 2) que torna $\Omega$ uma superfície de Riemann. No caso em que $\pi_{1}(\Omega)=\{0\}$ ou $\pi_{1}(\Omega)=\mathbb{Z}$, podemos utilizar teoremas de uniformização de superfícies de Riemann para "colar" as soluções locais de $L$, obtendo uma solução global que herda as boas propriedades das soluções locais.

Portanto, antes de tratar do caso hipoelítico, vamos tratar do caso elítico. Como eliticidade é uma hipótese mais forte sobre um campo, é natural pensar que tenhamos uma tese mais forte também, i.e., no capítulo 4 que trata do caso elítico, quando $L$ é um campo vetorial complexo elítico definido em um aberto conexo $\Omega \subset \mathbb{R}^{2}$, vamos mostrar que o problema

$\left(*^{\prime}\right) \quad L Z=0, \operatorname{grad}(\Re\{Z\}), \operatorname{grad}(\Im\{Z\})$ linearmente independentes e $Z$ injetiva em $\Omega$ tem solução global no caso em que $\pi_{1}(\Omega)=\{0\}$ ou $\pi_{1}(\Omega)=\mathbb{Z}$. Também será feito um pequeno estudo do caso semiglobal. 
No caso hipoelítico, uma alternativa para obter a integrabilidade local, que no caso elítico é obtida a partir do estudo das equações de Beltrami, é o estudo da resolubilidade local de operadores diferenciais parciais lineares hipoelíticos (tratado no capítulo 3). Como em geral um campo hipoelítico não é elítico, uma alternativa para obter uma estrutura diferencial complexa induzida em $\Omega$ por $L$, que no caso elítico é obtida da existência local de mudanças de coordenadas que transformam $L$ em um múltiplo do operador de CauchyRiemann, é a aplicação do teorema de aproximação de Baouendi-Treves, para obter o teorema de representação de soluções, que chamamos de estrutura local das soluções. 


\section{Capítulo}

\section{Pré-requisitos}

\subsection{Notação}

Seja $\mathbb{Z}_{+}$o conjunto dos números inteiros não negativos $\{0,1,2,3, \ldots\}$ e seja $\mathbb{Z}_{+}^{n}$ o conjunto das $n$-uplas de $\mathbb{Z}_{+}$. Um elemento de $\mathbb{Z}_{+}^{n}$ é chamado de multi-índice e denotado, em geral, pelas letras gregas minúsculas da seguinte forma: $\alpha=\left(\alpha_{1}, \ldots, \alpha_{n}\right), \beta=\left(\beta_{1}, \ldots, \beta_{n}\right)$, etc. Em $\mathbb{Z}_{+}^{n}$ definimos a soma e a multiplicação por um elemento de $\mathbb{Z}_{+}$:

$$
\alpha+\beta=\left(\alpha_{1}+\beta_{1}, \ldots, \alpha_{n}+\beta_{n}\right) \text { e } k \alpha=\left(k \alpha_{1}, \ldots k \alpha_{n}\right) .
$$

Denotamos por $|\alpha|$ a soma das coordenadas de $\alpha$ :

$$
|\alpha|=\sum_{j=1}^{n} \alpha_{j}, \text { para } \alpha \in \mathbb{Z}_{+}^{n}
$$

e por $\alpha$ ! o produto dos fatoriais das coordenadas:

$$
\alpha !=\prod_{j=1}^{n} \alpha_{j} !, \text { para } \alpha \in \mathbb{Z}_{+}^{n} .
$$

Também definimos uma relação de ordem, isto é, se $\alpha, \beta \in \mathbb{Z}_{+}^{n}$,

$$
\alpha \leq \beta \text { se } \alpha_{j} \leq \beta_{j} \text { para } 1 \leq j \leq n .
$$


Definimos

$$
\left(\begin{array}{l}
\alpha \\
\beta
\end{array}\right)=\prod_{j=1}^{n}\left(\begin{array}{c}
\alpha_{j} \\
\beta_{j}
\end{array}\right)=\prod_{j=1}^{n} \frac{\alpha_{j} !}{\left(\alpha_{j}-\beta_{j}\right) ! \beta_{j}}=\frac{\alpha !}{(\alpha-\beta) ! \beta !},
$$

se $\alpha \geq \beta$ e $\left(\begin{array}{c}\alpha \\ \beta\end{array}\right)=0$ se $\alpha<\beta$.

Dado $x=\left(x_{1}, \ldots, x_{n}\right) \in \mathbb{R}^{n}$, denotamos

$$
x^{\alpha}=\prod_{j=1}^{n} x_{j}^{\alpha_{j}}, \text { para } \alpha \in \mathbb{Z}_{+}^{n} .
$$

Se $1 \leq j \leq n$, definimos

$$
D_{x_{j}} \doteq \frac{1}{i} \frac{\partial}{\partial x_{j}}
$$

que pode ser denotado por $D_{j}$, onde $\frac{\partial}{\partial x_{j}}$ indica a derivada parcial em relação a $x_{j}$ em $\mathbb{R}^{n}$, que pode ser denotada por $\partial_{j}$.

Para $\alpha \in \mathbb{Z}^{n}$, definimos

$$
D_{x}^{\alpha}=\prod_{j=1}^{n} D_{x_{j}}{ }^{\alpha_{j}} .
$$

Obs. 1.1.1. Quando $\alpha=0$, então $\partial^{\alpha} f=f$.

Seja $\Omega \subset \mathbb{R}^{n}$ um aberto e seja $k \in \mathbb{Z}_{+}$. O espaço das funções de classe $C^{k}$ em $\Omega$, indicado por $C^{k}(\Omega)$, consiste de todas as $f$, funções definidas em $\Omega$ a valores complexos com derivadas contínuas até ordem $k$, isto é, $f \in C^{k}(\Omega)$ se, e somente se, $\partial^{\alpha} f$ é contínua para todo $\alpha \in \mathbb{Z}_{+}^{n} \operatorname{com}|\alpha| \leq k$. Assim $C^{0}(\Omega)$ denota o conjunto das funções a valores complexos em $\Omega$ que são contínuas. Analogamente, $C^{\infty}(\Omega)$, consiste das funções definidas em $\Omega$ a valores complexos com derivadas contínuas de todas as ordens. Mais explicitamente,

$$
C^{\infty}(\Omega)=\bigcap_{k \in \mathbb{Z}_{+}} C^{k}(\Omega)
$$


Definição 1.1.2. O suporte de uma função $f \in C^{0}(\Omega)$, indicado por $\operatorname{supp}(f)$, é o fecho em $\Omega$ do conjunto $\{x \in \Omega ; f(x) \neq 0\}$.

Obs. 1.1.3. O suporte de $f$ é o complementar em $\Omega$ do maior aberto onde $f$ é nula.

Indicamos por $C_{c}^{k}(\Omega)$, o subconjunto de $C^{k}(\Omega)$ das funções com suporte compacto e por $C_{c}^{\infty}(\Omega)$ o subconjunto de $C^{\infty}(\Omega)$ das funções de suporte compacto.

$$
C_{c}^{\infty}(\Omega)=\bigcap_{k \in \mathbb{Z}_{+}} C_{c}^{k}(\Omega)
$$

Obs. 1.1.4. $C^{k}(\Omega), C^{\infty}(\Omega), C_{c}^{k}(\Omega)$ e $C_{c}^{\infty}(\Omega)$ são espaços vetoriais sobre $\mathbb{C}$, com as operações usuais de funções.

O conjunto das funções localmente integráveis em $\Omega$, indicado por $L_{l o c}^{1}(\Omega)$, é o conjunto das funções $f$ Lebesgue mensuráveis em $\Omega$ tais que

$$
\int_{K}|f(x)| d x<\infty
$$

para todo $K \subset \Omega$ compacto.

Obs. 1.1.5. O conjunto $L_{\text {loc }}^{1}(\Omega)$ é um espaço vetorial sobre $\mathbb{C}$. Dizemos que uma sequência $\left(f_{j}\right)_{j \in \mathbb{N}}$ converge em $L_{l o c}^{1}(\Omega)$, quando existe uma função $f \in L_{l o c}^{1}(\Omega)$, tal que para todo $K \subset \Omega$ compacto

$$
\int_{K}\left|f_{j}(x)-f(x)\right| d x \longrightarrow 0, \text { quando } j \longrightarrow \infty
$$

O espaço $L_{\text {loc }}^{1}(\Omega)$ é completo, i.e., se $\left(f_{j}\right)_{j \in \mathbb{N}}$ é uma sequência de Cauchy em $L_{\text {loc }}^{1}(\Omega)$, então existe $f \in L_{l o c}^{1}(\Omega)$ tal que $f_{j} \longrightarrow f$ em $L_{l o c}^{1}(\Omega)$.

Com a notação multi-índice, a formula de Taylor com resto integral fica da forma:

$$
f(x+y)=\sum_{|\alpha|<k} \partial^{\alpha} f(x) \frac{y^{\alpha}}{\alpha !}+k \int_{0}^{1}(1-t)^{k-1} \sum_{|\alpha|=k} \partial^{\alpha} f(x+t y) \frac{y^{\alpha}}{\alpha !} d t
$$

se $f \in C^{k}$ em uma vizinhança aberta do segmento $[x, x+y]$. 


\subsection{Espaços Vetoriais Topológicos}

Seja $\mathbf{E}$ um espaço vetorial sobre $\mathbb{C}$. Uma topologia $\mathscr{T}$ em $\mathbf{E}$ é dita compatível com a estrutura vetorial se as operações

$$
\begin{aligned}
& \mathbf{E} \times \mathbf{E} \longrightarrow \mathbf{E} \quad \mathbb{C} \times \mathbf{E} \longrightarrow \mathbf{E} \\
& (x, y) \longmapsto x+y \quad(\lambda, x) \longmapsto \lambda x
\end{aligned}
$$

são contínuas. O espaço $\mathbf{E}$ provido de uma topologia que preserva a estrutura vetorial é chamado de Espaço Vetorial Topológico, e é indicado pela abreviação EVT.

Segue que se E é um EVT, para cada $a \in \mathbf{E}$ e $\lambda \neq 0$ fixados, as aplicações

$$
x \mapsto a+x \quad e \quad x \mapsto \lambda x
$$

são homeomorfismos. Seja E um EVT. Para especificar sua topologia, basta dar uma base de vizinhanças de 0 (elemento nulo do espaço $\mathbf{E}$ ). Esta é uma família $\mathscr{B}$ de vizinhanças de 0 tal que toda vizinhança de 0 contém algum elemento de $\mathscr{B}$. Assim um conjunto $G \subset \mathbf{E}$ é aberto se, e somente se,

$$
\text { para cada } x \in G, \exists U \in \mathscr{B} \text { tal que } x+U \subset G \text {. }
$$

Um aberto $U$ de $\mathbf{E}$ é:

- Convexo: Se $x, y \in U$ e $0 \leq t \leq 1 \Longrightarrow x t+(1-t) y \in U$.

- Equilibrado: Se $x \in U,|c|<1 \operatorname{com} c \in \mathbb{C} \Longrightarrow c x \in U$.

- Absorvente: $\forall x \in \mathbf{E}, \exists t=t_{x}>0$ tal que $x \in t U=\{t y ; y \in U\}$.

Um EVT é localmente convexo se é Hausdorff e existe uma base de vizinhanças de 0 formada por conjuntos abertos que são convexos, equilibrados e absorventes. Um EVT localmente convexo é indicado pela abreviação ELC. 
Existe um modo muito importante de construir um ELC. Vamos falar um pouco disso agora.

Definição 1.2.1. Uma seminorma em um espaço vetorial $\boldsymbol{E}$ é uma função

$$
p: \boldsymbol{E} \longrightarrow \mathbb{R}
$$

tal que:

1. $p(c x)=|c| p(x), c \in \mathbb{C}, x \in \boldsymbol{E}$;

2. $p(x+y) \leq p(x)+p(y), x, y \in \boldsymbol{E}$.

Obs. 1.2.2. Segue de 1 que $p(0)=0$. De 2 temos

$$
0=p(x-x) \leq p(x)+p(x)=2 p(x), \forall x \in \boldsymbol{E} .
$$

Ou seja, $p(x) \geq 0, \forall x \in \boldsymbol{E}$.

Uma família $\mathscr{P}$ de seminormas em $\mathbf{E}$ é dita separante se para todo $x \in \mathbf{E}, \operatorname{com} x \neq 0$, existe $p \in \mathscr{P}$ tal que $p(x) \neq 0$. Dada uma tal família $\mathscr{P}$, seja $\mathscr{B}$ a coleção de todos os conjuntos da forma

$$
\{x \in \mathbf{E} ; p(x)<\epsilon\}, \operatorname{com} \epsilon>0, p \in \mathscr{P}
$$

e das interseções finitas de tais conjuntos. Então prova-se que $\mathscr{B}$ é uma base de vizinhanças de 0 em uma topologia que torna $\mathbf{E}$ um espaço vetorial topológico localmente convexo. Esta topologia é chamada: a topologia gerada pelas seminormas em $\mathscr{P}$.

Obs. 1.2.3. Seja $\boldsymbol{E}$ um $\boldsymbol{E L C}$. Se U é uma vizinhança de 0 que é convexa, equilibrada e absorvente, então prova-se

$$
p_{U}(x)=\inf \{t>0 ; x \in t U\}
$$

chamado de funcional de Minkowski de U, é uma seminorma de $\boldsymbol{E}$, e que a topologia de $\boldsymbol{E}$ pode ser gerada pela familia de seminormas desta forma. 
Seja agora $\mathscr{P}$ uma família enumerável de seminormas, digamos $\mathscr{P}=\left\{p_{1}, p_{2}, \cdots\right\}$. Para que seja separante é preciso que valha:

$$
x \in \mathbf{E}, \quad p_{k}(x)=0, \quad \forall k \in \mathbb{N} \Longrightarrow x=0 .
$$

A topologia gerada pela família de seminormas é metrizavel. Por exemplo:

$$
d(x, y) \doteq \sum_{k=1}^{\infty} \frac{1}{2^{k}} \frac{p_{k}(x-y)}{1+p_{k}(x-y)}, \quad x, y \in \mathbf{E}
$$

é uma métrica que define a mesma topologia que a família de seminormas $\mathscr{P}$ e é invariante por translações.

Obs. 1.2.4. Note que (1.2.2) é crucial para mostrar que d é uma métrica.

Reciprocamente, pode-se provar que a topologia de um ELC com base enumerável é metrizável e tem uma métrica invariante por translações que pode ser gerada por uma família enumerável de seminormas.

Para nós um Espaço de Fréchet é um ELC cuja topologia é definida por uma família enumerável separante de seminormas as quais definem a métrica como em (1.2.3) e o espaço com esta métrica é completo.

Em termos de uma família enumerável de seminormas, que geram a topologia, digamos $\left\{p_{1}, p_{2}, p_{3}, \cdots\right\}$, podemos enunciar um resultado como segue. Seja $\left(x_{k}\right)$ uma sequência em E. Se $p_{m}\left(x_{j}-x_{l}\right) \longrightarrow 0$, quando $j, l \longrightarrow \infty, \forall m \in \mathbb{N}$, então $\exists x \in \mathbf{E}$ tal que $p_{m}\left(x_{j}-x\right) \longrightarrow 0$, quando $j \longrightarrow \infty, \forall m \in \mathbb{N}$.

Exemplo 1.2.5. Seja $\Omega \subset \mathbb{R}^{n}$ um aberto. Seja $\left(\Omega_{j}\right)_{j \in \mathbb{N}}$ tal que $\Omega_{j} \subset \Omega$ é aberto com $\overline{\Omega_{j}}$ compacto, $\overline{\Omega_{j}} \subset \Omega_{j+1}$, para todo $j \in \mathbb{N} e \bigcup_{j \in \mathbb{N}} \Omega_{j}=\Omega$.

O espaço vetorial $C^{\infty}(\Omega)$ com a topologia gerada pela familia de seminormas

$$
p_{k, j}(\varphi)=\sum_{|\alpha| \leq k} \sup _{\Omega_{j}}\left|\partial^{\alpha} \varphi\right|
$$


é um espaço de Fréchet.

Obs. 1.2.6. A sequência $\left(\Omega_{j}\right)_{j \in \mathbb{N}}$ é chamada de sequência de abertos esgotantes de $\Omega$.

Exemplo 1.2.7. Seja $K \subset \Omega$ compacto. O espaço vetorial

$$
C_{c}^{\infty}(K)=\left\{\varphi \in C^{\infty}(\Omega) ; \operatorname{supp}(\varphi) \subset K\right\}
$$

com a familia de seminormas

$$
P_{k}(\varphi)=\sum_{|\alpha| \leq k} \sup _{K}\left|\partial^{\alpha} \varphi\right|, k \in \mathbb{Z}_{+}
$$

é um espaço de Fréchet.

Obs. 1.2.8. Espaços de Fréchet são importantes porque certas consequências de Teorema de Baire são válidas neles. Por exemplo: Teorema da aplicação aberta dado pelo teorema abaixo.

Teorema 1.2.9. Sejam $\boldsymbol{E}, \boldsymbol{F}$ dois espaços de Fréchet. Se $T: \boldsymbol{E} \longrightarrow \boldsymbol{F}$ é uma aplicação linear contínua e sobrejetiva, então $T$ é aberta.

Em particular, quando $T: \mathbf{E} \longrightarrow \mathbf{F}$ é uma bijeção, então $T^{-1}: \mathbf{F} \longrightarrow \mathbf{E}$ é contínua.

Proposição 1.2.10. Seja $\boldsymbol{E}$ um $\boldsymbol{E} \boldsymbol{L} \boldsymbol{C}$ e seja $\mathscr{P}$ uma familia de seminormas que gera a topologia de $\boldsymbol{E}$. Então a seminorma q é contínua em $\boldsymbol{E}$ se, e somente se, existe um subconjunto finito $\left\{p_{1}, p_{2}, \ldots, p_{N}\right\} \subset \mathscr{P}$ e uma constante $C$ tal que

$$
q(x) \leq C \max \left\{p_{1}(x), \ldots, p_{N}(x)\right\}, \quad x \in \boldsymbol{E} .
$$

\section{Demonstração:}

Suponha $q$ contínua. Então $q^{-1}(-1,1)$ é um aberto de $\mathbf{E}$ que contém 0 . Logo existe um aberto

$$
A=\bigcap_{j=1}^{N}\left\{y \in \mathbf{E} ; p_{j}(y)<\epsilon_{j}, p_{j} \in \mathscr{P}, \epsilon_{j}>0\right\}
$$

tal que $A \subset q^{-1}(-1,1)$. Seja $x \in \mathbf{E}$. Se $p_{j}(x)>0$ para algum $j \in\{1,2, \ldots, N\}$, então tomando 


$$
t=\frac{1}{2} \frac{\min \left\{\epsilon_{1}, \ldots, \epsilon_{N}\right\}}{\max \left\{p_{1}(x), \ldots, p_{N}(x)\right\}}
$$

temos $t x \in A \subset q^{-1}(-1,1)$. Assim $q(t x)<1$. Logo

$$
q(x)<\frac{1}{t}=2 \frac{\max \left\{p_{1}(x), \ldots, p_{N}(x)\right\}}{\min \left\{\epsilon_{1}, \ldots, \epsilon_{N}\right\}}=C \max \left\{p_{1}(x), \ldots, p_{N}(x)\right\},
$$

com $C=2 / \min \left\{\epsilon_{1}, \ldots, \epsilon_{N}\right\}$. Agora, se $p_{j}(x)=0$, para todo $j \in\{1, \ldots, N\}$, então $p_{j}(t x)=0$, para todo $j \in\{1, \ldots, N\}$, para todo $t>0$, ou seja, $t x \in A$, para todo $t>0$. Logo $q(t x)<1$, para todo $t>0$. Daí temos $0 \leq q(x)<\frac{1}{t}$, para todo $t>0$. Segue que $q(x)=0$ e assim (1.2.4) é trivialmente satisfeita.

Reciprocamente, suponha que exista um subconjunto finito de $\mathscr{P}$ de modo que (1.2.4) seja satisfeita. Então $q$ é contínua no 0 . Como $q$ é uma seminorma em E, temos que $q$ satisfaz

$$
|q(x)-q(y)| \leq q(x-y), \quad x, y \in \mathbf{E} .
$$

Daí, segue que $q$ é contínua em E.

Obs. 1.2.11. Um corolário da proposição acima, é que a topologia de $\boldsymbol{E}$ é gerada pela familia de todas as seminormas continuas em $\boldsymbol{E}$.

Corolário 1.2.12. Seja $\boldsymbol{E}$ como na proposição acima. Então um funcional linear u em $\boldsymbol{E}$ é contínuo se, e somente se, existe um subconjunto finito $\left\{p_{1}, \ldots, p_{N}\right\} \subset \mathscr{P}$ e uma constante $C$ tais que

$$
|\langle u, x\rangle| \leq C \max \left\{p_{1}(x), \ldots, p_{N}(x)\right\}, x \in \boldsymbol{E} .
$$

\section{Demonstração:}

Seja $u$ um funcional linear em $\mathbf{E}$. Então $\mathbf{E} \ni x \mapsto|\langle u, x\rangle|$ é uma seminorma em $\mathbf{E}$. Assim o resultado segue diretamente da proposição 1.2.10. 
Proposição 1.2.13. Sejam $\boldsymbol{E}$ e $\boldsymbol{F}$ dois $\boldsymbol{E L}$ C e sejam $\mathscr{P}$ e $\mathscr{Q}$ duas familias de seminormas que geram as topologias de $\boldsymbol{E}$ e $\boldsymbol{F}$ respectivamente. Então a aplicação linear $T: E \longrightarrow F$ é continua se, e somente se, para toda $q \in \mathscr{Q}$, existe um subconjunto finito $\left\{p_{1}, p_{2}, \ldots, p_{N}\right\} \subset$ $\mathscr{P}$ e uma constante $C=C(q)$ tal que

$$
q(T(x)) \leq C \max \left\{p_{1}(x), \ldots, p_{N}(x)\right\}, x \in \boldsymbol{E} .
$$

Demonstração: O argumento desta demostração é analogo ao argumento usado na demontração da proposição 1.2.10.

Obs. 1.2.14. Existe uma tecnica em análise para obter desigualdades que é a aplicação do teorema 1.2.9 juntamente com a proposição 1.2.13 a espaços de Fréchet adequados. Essa tecnica será usada na demonstração do teorema 3.3.1.

\subsubsection{A topologia de $C_{c}^{\infty}(\Omega)$ e o espaço $\mathscr{D}^{\prime}(\Omega)$}

Vamos falar agora um pouco sobre a topologia usual ou natural de $C_{c}^{\infty}(\Omega)$. Esta topologia é chamada de Topologia limite indutivo dos espaços $C_{c}^{\infty}(K), K \subset \Omega$ compacto. O próximo teorema trata da topologia limite indutivo de maneira geral.

Teorema 1.2.15. Seja $\boldsymbol{E}$ um espaço vetorial complexo (ou real). Seja $\left\{\boldsymbol{E}_{j}\right\}_{j \in \mathbb{N}}$ uma familia de subespaços com

$$
\boldsymbol{E}_{j} \subset \boldsymbol{E}_{j+1}, \boldsymbol{E}=\bigcup_{j \in \mathbb{N}} \boldsymbol{E}_{j}
$$

Suponha que cada $\boldsymbol{E}_{j}$ possui uma topologia localmente convexa e que a topologia de $\boldsymbol{E}_{j}$ coincide com a topologia induzida por $\boldsymbol{E}_{j+1}$. Seja $\mathscr{B}$ a coleção dos conjuntos equilibrados, absorventes e convexos $V$ em $\boldsymbol{E}$ tais que $V \cap \boldsymbol{E}_{j}$ é um aberto em $\boldsymbol{E}_{j}$ para cada $j \in \mathbb{N}$. Então:

1. $\mathscr{B}$ é uma base de vizinhanças do 0 para uma topologia localmente convexa.

2. A topologia gerada por $\mathscr{B}$ é a topologia localmente convexa mais fina em $\boldsymbol{E}$ que torna continua as inclusões $\boldsymbol{E}_{j} \longrightarrow \boldsymbol{E}$, para todo $j \in \mathbb{N}$. 
3. A topologia induzida por $\boldsymbol{E}$ em $\boldsymbol{E}_{j}$ coincide com a topologia de $\boldsymbol{E}_{j}$.

A demonstração deste teorema pode ser encontrada em [25].

O espaço $\mathbf{E}$ com a topologia gerada por $\mathscr{B}$ é chamado de limite indutivo dos espaços $\mathbf{E}_{j}$.

Agora, seja $\Omega$ um aberto conexo de $\mathbb{R}^{n}$. Seja $C_{c}^{\infty}(\Omega)$ o conjunto das funções infinitamente diferenciáveis com suporte em $\Omega$. Seja $\left\{K_{j}\right\}_{j \in \mathbb{N}}$ uma sequência de conjuntos compactos de $\Omega$ tais que

$$
K_{j} \subset K_{j+1}, \bigcup_{j \in \mathbb{N}} K_{j}=\Omega
$$

Para cada $j \in \mathbb{N}$ considere o espaço de Fréchet $C_{c}^{\infty}\left(K_{j}\right)$ como no exemplo 1.2.7. Observe que $\left\{C_{c}^{\infty}\left(K_{j}\right)\right\}_{j \in \mathbb{N}}$ é uma famíla de subespaços de $C_{c}^{\infty}(\Omega)$ que satisfaz as hipóteses do teorema 1.2 .15 e assim a topologia em $C_{c}^{\infty}(\Omega)$ definida pelo limite indutivo dos espaços $C_{c}^{\infty}\left(K_{j}\right)$ é a que chamamos de topologia usual de $C_{c}^{\infty}(\Omega)$.

Obs. 1.2.16. A topologia usual de $C_{c}^{\infty}(\Omega)$ não depende da sequência $\left\{K_{j}\right\}_{j \in \mathbb{N}}$ de compactos de $\Omega$.

\section{Critério de Convergência em $C_{c}^{\infty}(\Omega)$}

Uma sequência $\left(\varphi_{j}\right)_{j \in \mathbb{N}}$ converge para zero em $C_{c}^{\infty}(\Omega)$ se, e somente se, existe um compacto $K \subset \Omega$ tal que:

1. $\operatorname{supp}\left(\varphi_{j}\right) \subset K$, para todo $j \in \mathbb{N}$;

2. para todo $\alpha \in \mathbb{Z}_{+}^{n}$, a sequência $\left(\partial^{\alpha} \varphi_{j}\right)_{j \in \mathbb{N}}$ converge uniformemente para zero em $K$.

Definição 1.2.17. Uma distribuição em $\Omega$ é um funcional linear contínuo em $C_{c}^{\infty}(\Omega)$.

Denotamos por $\mathscr{D}^{\prime}(\Omega)$ o conjunto de todas as distribuições em $\Omega$. A ação de $u \in \mathscr{D}^{\prime}(\Omega)$ sobre $\varphi \in C_{c}^{\infty}(\Omega)$ é indicada por $\langle u, \varphi\rangle$.

Teorema 1.2.18. Um funcional linear em $C_{c}^{\infty}(\Omega)$ é contínuo se, e somente se, para cada compacto $K \subset \Omega$, existe uma constante $C$ e um inteiro positivo $m$ tais que 


$$
|\langle u, \varphi\rangle| \leq C \sum_{|\alpha| \leq m} \sup _{K}\left|\partial^{\alpha} \varphi\right|
$$

para toda $\varphi \in C_{c}^{\infty}(K)$.

\section{Demonstração:}

Seja $u \in \mathscr{D}^{\prime}(\Omega)$. Como $u$ é um funcional linear contínuo em $C_{c}^{\infty}(\Omega)$ e para cada $K \subset \Omega$ compacto a inclusão $C_{c}^{\infty}(K) \longrightarrow C_{c}^{\infty}(\Omega)$ é contínua, temos que a restrição de $u$ a $C_{c}^{\infty}(K)$ é contínua. Portanto pelo corolário 1.2 .12 existem $k_{1}, \ldots, k_{N} \in \mathbb{Z}_{+}$e $C>0$ tais que

$$
|\langle u, \varphi\rangle| \leq C \max \left\{P_{k_{1}}(\varphi), \ldots, P_{k_{N}}(\varphi)\right\}, \text { para toda } \varphi \in C_{c}^{\infty}(K),
$$

onde $P_{k}, k \in \mathbb{Z}^{+}$, denotam a família de seminormas que gera a topologia de $C_{c}^{\infty}(K)$ discutido no exemplo 1.2.7. Daí,

$$
|\langle u, \varphi\rangle| \leq C \sum_{j=1}^{N} P_{k_{j}}(\varphi) \leq C \sum_{|\alpha| \leq m} \sup _{K}\left|\partial^{\alpha} \varphi\right|,
$$

para toda $\varphi \in C_{c}^{\infty}(K)$, onde $m=\max \left\{k_{1}, \ldots, k_{N}\right\}$.

Reciprocamente, suponha que para cada compacto $K \subset \Omega$, existe uma constante $C$ e um inteiro positivo $m$ tais que

$$
|\langle u, \varphi\rangle| \leq C \sum_{|\alpha| \leq m} \sup _{K}\left|\partial^{\alpha} \varphi\right|,
$$

para toda $\varphi \in C_{c}^{\infty}(K)$. Então para cada $K \subset \Omega$ a restrição de $u$ a $C_{c}^{\infty}(K)$ é contínua. Logo $u$ é contínuo em $C_{c}^{\infty}(\Omega)$. Ou seja, $u \in \mathscr{D}^{\prime}(\Omega)$.

Vamos ver agora alguns exemplos de distribuições

Exemplo 1.2.19. Seja $f \in L_{l o c}^{1}(\Omega)$. Então

$$
\left\langle u_{f}, \varphi\right\rangle=\int_{\Omega} f(x) \varphi(x) d x, \varphi \in C_{c}^{\infty}(\Omega),
$$

define uma distribuição em $\Omega$. 
Obs. 1.2.20. A aplicação $L_{l o c}^{1}(\Omega) \ni f \mapsto u_{f} \in \mathscr{D}^{\prime}(\Omega)$ é linear e injetora. Logo $L_{l o c}^{1}(\Omega)$ pode ser considerado como um subespaço de $\mathscr{D}^{\prime}(\Omega)$. Devido a isso, a distribuição $u_{f}$ será denotada por $f$. Em particular, toda função de classe $C^{k}(\Omega), k=0,1,2, \ldots, \infty$ define uma distribuição em $\Omega$.

Exemplo 1.2.21. A medida de Dirac no ponto $a \in \Omega$ define por meio de

$$
\left\langle\delta_{a}, \varphi\right\rangle=\varphi(a), \varphi \in C_{c}^{\infty}(\Omega)
$$

uma distribuição em $\Omega$.

Dizemos que uma distribuição $u \in \mathscr{D}^{\prime}(\Omega)$ é nula num aberto $V \subset \Omega$ se $\langle u, \varphi\rangle=0$, para toda $\varphi \in C_{c}^{\infty}(V)$.

Definição 1.2.22. Se $u \in \mathscr{D}^{\prime}(\Omega)$ definimos o suporte de $u, \operatorname{supp}(u)$, como a interseção de todos os fechados de $\Omega$ fora dos quais u é nula.

Exemplo 1.2.23. $\operatorname{supp}\left(\delta_{a}\right)=\{a\}$.

Exemplo 1.2.24. Se $f$ é uma função contínua em $\Omega$, então $f$ define uma distribuição em $\Omega$ como elemento de $L_{l o c}^{1}(\Omega)$. Seu suporte, como função, coincide com seu suporte, como distribuição. Isto é, na notação do exemplo 1.2.19,

$$
\operatorname{supp}(f)=\operatorname{supp}\left(u_{f}\right)
$$

Em particular, quando $f$ é contínua em $\Omega$ e $\operatorname{supp}\left(u_{f}\right)$ tem medida de Lebesgue nula em $\Omega$, então $f$ é uma função identicamente nula em $\Omega$.

Inspirado no exemplo 1.2.19, uma distribuição $u \in \mathscr{D}^{\prime}(\Omega)$ é dita $C^{\infty}$ no aberto $U \subset \Omega$, se existe uma função $f \in C^{\infty}(U)$ tal que

$$
\langle u, \varphi\rangle=\int_{U} f(x) \varphi(x) d x, \text { para toda } \varphi \in C_{c}^{\infty}(U) .
$$

Definição 1.2.25. Se $u \in \mathscr{D}^{\prime}(\Omega)$ definimos o suporte singular de $u$, $\operatorname{suppsing}(u)$, como a interseção de todos os fechados de $\Omega$ fora dos quais u é $C^{\infty}$. 
Derivação e multiplicação por funções $C^{\infty}$

Definição 1.2.26. Se $u \in \mathscr{D}^{\prime}(\Omega)$ e $\alpha \in \mathbb{Z}_{+}^{n}$, definimos $\partial^{\alpha} u \in \mathscr{D}^{\prime}(\Omega)$ por

$$
\left\langle\partial^{\alpha} u, \varphi\right\rangle \doteq(-1)^{|\alpha|}\left\langle u, \partial^{\alpha} \varphi\right\rangle, \text { para toda } \varphi \in C_{c}^{\infty}(\Omega)
$$

Exemplo 1.2.27. Seja $H \in L_{l o c}^{1}(\mathbb{R})$ a função de Heaviside dada por

$$
H(x)=\left\{\begin{array}{lll}
1, & \text { se } & x>0 \\
0, & \text { se } & x \leq 0
\end{array}\right.
$$

Então $H^{\prime}=\delta$.

Definição 1.2.28. Se $u \in \mathscr{D}^{\prime}(\Omega)$ e $f \in C^{\infty}(\Omega)$, definimos $f u \in \mathscr{D}^{\prime}(\Omega)$ por

$$
\langle f u, \varphi\rangle \doteq\langle u, f \varphi\rangle, \text { para toda } \varphi \in C_{c}^{\infty}(\Omega) .
$$

Exemplo 1.2.29. Se $0 \in \Omega$ e $f \in C^{\infty}(\Omega)$ é tal que $f(0)=0$, então $f \delta_{0}=0$.

Obs. 1.2.30. Vale a Regra de Leibniz: se $u \in \mathscr{D}^{\prime}(\Omega), f \in C^{\infty}(\Omega)$ e $\alpha \in \mathbb{Z}_{+}^{n}$, então

$$
\partial^{\alpha}(f u)=\sum_{\beta \leq \alpha}\left(\begin{array}{l}
\alpha \\
\beta
\end{array}\right) \partial^{\alpha-\beta} f \partial^{\beta} u .
$$




\subsubsection{Mudança de Variáveis em Distribuições}

Sejam $\Omega_{1}, \Omega_{2} \subset \mathbb{R}^{2}$ abertos.

Seja $F: \Omega_{1} \longrightarrow \Omega_{2}$ um difeomorfismo $C^{\infty}$. Definimos a aplicação

$$
\begin{aligned}
F^{*}: C^{\infty}\left(\Omega_{2}\right) & \longrightarrow C^{\infty}\left(\Omega_{1}\right) \\
u & \longmapsto u \circ F
\end{aligned}
$$

Note que $F^{*}$ é bijetora e linear. Se $U \subset \Omega_{1}$ e $V \subset \Omega_{2}$ são abertos tais que $F(U)=V$, então a restrição de $F^{*}$ a $C^{\infty}(V)$ é uma bijeção linear de $C^{\infty}(V)$ em $C^{\infty}(U)$. Observe também que se $f, g \in C^{\infty}\left(\Omega_{2}\right)$, então $F^{*}(f g)=F^{*}(f) F^{*}(g)$. Temos assim que $F^{*}$ é um isomorfismo entre os espaços vetoriais $C^{\infty}\left(\Omega_{2}\right)$ e $C^{\infty}\left(\Omega_{1}\right)$. A aplicação $F^{*}$ é chamada de pullback.

Teorema 1.2.31. A aplicação $F^{*}$ se estende a uma aplicação linear contínua $F^{*}$ : $\mathscr{D}^{\prime}\left(\Omega_{2}\right) \longrightarrow \mathscr{D}^{\prime}\left(\Omega_{1}\right)$. Além disso $F^{*}$ é uma bijeção com inversa $\left(F^{-1}\right)^{*}$.

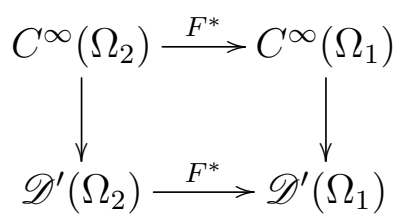

As setas verticais representam a aplicação inclusão:

$$
C^{\infty}\left(\Omega_{j}\right) \ni f \mapsto u_{f} \in \mathscr{D}^{\prime}\left(\Omega_{j}\right), j=1,2 .
$$

\section{Demonstração:}

A continuidade de $F^{*}: \mathscr{D}^{\prime}\left(\Omega_{2}\right) \longrightarrow \mathscr{D}^{\prime}\left(\Omega_{1}\right)$ é em relação à convergência fraca.

A fim de obter uma extensão de $F^{*}$ como a do enunciado do teorema, sejam $u \in$ $C^{\infty}\left(\Omega_{2}\right)$ e $\varphi \in C_{c}^{\infty}\left(\Omega_{1}\right)$. Olhando para $u$ como uma distribuição, temos

$$
\left\langle F^{*}(u), \varphi\right\rangle=\int u(F(x)) \varphi(x) d x=\int u\left(x^{\prime}\right) \varphi\left(F^{-1}\left(x^{\prime}\right)\right)\left|\operatorname{det}\left[\left(F^{-1}\right)^{\prime}\right]\left(x^{\prime}\right)\right| d x^{\prime}
$$




$$
=\left\langle u,\left|\operatorname{det}\left[\left(F^{-1}\right)^{\prime}\right]\right|\left(\varphi \circ F^{-1}\right)\right\rangle \text {. }
$$

Dada então $u \in \mathscr{D}^{\prime}\left(\Omega_{2}\right)$ definimos

$$
F^{*}(u): C_{c}^{\infty}\left(\Omega_{1}\right) \longrightarrow \mathbb{C}
$$

dada por

$$
F^{*}(u)(\varphi)=u\left(\left|\operatorname{det}\left[\left(F^{-1}\right)^{\prime}\right]\right|\left(\varphi \circ F^{-1}\right)\right)
$$

$\varphi \in C_{c}^{\infty}\left(\Omega_{1}\right)$. É fácil ver que $F^{*}(u)$ é linear. Vamos mostrar que $F^{*}(u)$ é contínuo (i.e., $\left.F^{*}(u) \in \mathscr{D}^{\prime}\left(\Omega_{1}\right)\right)$. Para isso, seja $\left(\varphi_{j}\right)_{j \in \mathbb{N}}$ uma sequência em $C_{c}^{\infty}\left(\Omega_{1}\right)$ tal que $\varphi_{j} \longrightarrow 0$ em $C_{c}^{\infty}\left(\Omega_{1}\right)$. Isto é, existe um compacto $K \subset \Omega_{1}$ tal que $\operatorname{supp}\left(\varphi_{j}\right) \subset K$, para todo $j \in \mathbb{N}$ e $\partial^{\alpha} \varphi_{j} \longrightarrow 0$ uniformemente para todo $\alpha \in \mathbb{Z}_{+}^{n}$. Considere a sequência $\left(\phi_{j}\right)_{j \in \mathbb{N}}$ em $C_{c}^{\infty}\left(\Omega_{2}\right)$, onde $\phi_{j} \doteq\left|\operatorname{det}\left[\left(F^{-1}\right)^{\prime}\right]\right|\left(\varphi_{j} \circ F^{-1}\right), j \in \mathbb{N}$. Note que $F^{*}(u)\left(\varphi_{j}\right)=u\left(\phi_{j}\right)$, para todo $j \in \mathbb{N}$. Vamos mostrar que $\phi_{j} \longrightarrow 0$ em $C_{c}^{\infty}\left(\Omega_{2}\right)$. Para isso, seja $\alpha \in \mathbb{Z}_{+}^{n}$. Temos que $\partial^{\alpha} \phi_{j}$ é uma soma finita de produtos de derivadas das funções, $\left|\operatorname{det}\left[\left(F^{-1}\right)^{\prime}\right]\right|$, $\varphi_{j} \circ F^{-1}$ e derivadas das funções coordenadas de $F^{-1}$. Em particular, cada termo dessa soma possui em sua decomposição em produto, um termo da forma $\left(\partial^{\beta} \varphi_{j}\right) \circ F^{-1}, \beta \leq \alpha$. Daí, cada termo dessa soma tem suporte em $F(K)$ e converge uniformemente para zero em $F(K)$. Assim $\operatorname{supp}\left(\phi_{j}\right) \subset F(K)$ e novamente, como se trata de uma soma finita, temos que $\partial^{\alpha} \phi_{j} \longrightarrow 0$ uniformemente em $F(K)$. Portanto, $\phi_{j} \longrightarrow 0$ em $C_{c}^{\infty}\left(\Omega_{2}\right)$. Como $u \in \mathscr{D}^{\prime}\left(\Omega_{2}\right)$, temos $u\left(\phi_{j}\right) \longrightarrow 0(\mathrm{em} \mathbb{C})$. Ou seja, $F^{*}(u)\left(\varphi_{j}\right) \longrightarrow 0$. Portanto $F^{*}(u)$ é contínua, i.e., $F^{*}(u) \in \mathscr{D}^{\prime}\left(\Omega_{1}\right)$. Concluímos assim que $F^{*}: \mathscr{D}^{\prime}\left(\Omega_{2}\right) \longrightarrow \mathscr{D}^{\prime}\left(\Omega_{1}\right)$ está bem definida. Agora vamos mostrar sua continuidade. Seja $u_{j} \in \mathscr{D}^{\prime}\left(\Omega_{2}\right), j \in \mathbb{N}$ tal que $u_{j} \longrightarrow 0$ em $\mathscr{D}^{\prime}\left(\Omega_{2}\right)$. Dada $\varphi \in C_{c}^{\infty}\left(\Omega_{1}\right)$, temos $\left|\operatorname{det}\left[\left(F^{-1}\right)^{\prime}\right]\right|\left(\varphi \circ F^{-1}\right) \in C_{c}^{\infty}\left(\Omega_{2}\right)$. Logo $u_{j}\left(\left|\operatorname{det}\left[\left(F^{-1}\right)^{\prime}\right]\right|\left(\varphi \circ F^{-1}\right)\right) \longrightarrow 0($ em $\mathbb{C})$. Ou seja, $F^{*}\left(u_{j}\right)(\varphi) \longrightarrow 0$ para toda $\varphi \in C_{c}^{\infty}\left(\Omega_{1}\right)$. Assim $F^{*}\left(u_{j}\right) \longrightarrow 0$ em $\mathscr{D}^{\prime}\left(\Omega_{1}\right)$. Segue daí que $F^{*}$ é contínua.

Por fim, como $F$ é um difeomorfismo, temos assim que $\left(F^{-1}\right)^{*}: \mathscr{D}^{\prime}\left(\Omega_{1}\right) \longrightarrow \mathscr{D}^{\prime}\left(\Omega_{2}\right)$ está bem definido, e é fácil ver que $\left(F^{-1}\right)^{*}$ é a inversa de $F$. 


\subsection{Operadores Diferenciais Parciais Lineares}

Um operador diferencial parcial linear $(\boldsymbol{O D P L})$ em um aberto $\Omega$ de $\mathbb{R}^{n}$ é um operador da forma

$$
P(x, D)=\sum_{|\alpha| \leq m} a_{\alpha}(x) D^{\alpha}
$$

onde os coeficientes $a_{\alpha}(x) \quad(|\alpha| \leq m)$ são funções $C^{\infty}$ a valores complexos definidas em $\Omega$. Quando os coeficientes $a_{\alpha}(x)$ são constantes, denotaremos o operador por $P(D)$. Se para algum $|\alpha|=m$ existir um coeficiente $a_{\alpha}$ não identicamente nulo em $\Omega$, então diremos que $m$ é a ordem de $P(x, D)$. A parte principal de $P(x, D)$ é o operador obtido omitindo os termos de ordem menor que $m$ :

$$
P_{m}(x, D)=\sum_{|\alpha|=m} a_{\alpha}(x) D^{\alpha}
$$

O polinômio

$$
P_{m}(x, \xi)=\sum_{|\alpha|=m} a_{\alpha}(x) \xi^{\alpha}
$$

de grau $m$ com respeito a $\xi \in \mathbb{R}^{n}$, é chamado de símbolo principal de $P$.

No sentido clássico um ODPL em $\Omega$ de ordem $m$ está definido no espaço $C^{m}(\Omega)$. Mas, usando a derivação de distribuições e a multiplicação de uma distribuição por uma função $C^{\infty}$, podemos considerar um ODPL em $\Omega$ como sendo um operador de $\mathscr{D}^{\prime}(\Omega)$ em $\mathscr{D}^{\prime}(\Omega)$.

Definição 1.3.1. Dizemos que $P(x, D)$ é de tipo principal se satisfaz a seguinte condição:

$$
\begin{gathered}
\text { Para todo } x \in \Omega \text {, e } \xi \in \mathbb{R}^{n}, \xi \neq 0, \\
d_{\xi} P_{m}(x, \xi) \neq 0 .
\end{gathered}
$$

Exemplo 1.3.2. Um ODPL de ordem 1

$$
P(x, D)=\sum_{j=1}^{n} a_{j}(x) D_{x_{j}}+a_{0}(x)
$$


é de tipo principal em $\Omega$ se, e somente se,

$$
\sum_{j=1}^{n}\left|a_{j}(x)\right| \neq 0
$$

para todo $x \in \Omega$.

Definição 1.3.3. Um operador diferencial parcial linear $P$ de ordem $m$ é chamado elítico de ordem $m$ em $x$ se, e somente se,

$$
P_{m}(x, \xi) \neq 0 \text { se } \xi \in \mathbb{R}^{n} \text { e } \xi \neq 0
$$

$P$ é chamado de elítico em $\Omega$ se é elítico em todo $x \in \Omega$.

Se $P$ é elítico em $\Omega$, então $P$ é de tipo principal em $\Omega$.

Definição 1.3.4. Um operador diferencial parcial linear $P$ é chamado hipoelítico em $\Omega$ se, e somente se,

$$
\operatorname{suppsing}(P u)=\operatorname{suppsing}(u), \forall u \in \mathscr{D}^{\prime}(\Omega) \text {. }
$$

Em outras palavras, $P$ é hipoelítico em $\Omega$, quando para toda $u \in \mathscr{D}^{\prime}(\Omega)$ e $U \subset \Omega$ aberto se tem:

$$
P u \in C^{\infty}(U) \text {, então } u \in C^{\infty}(U) .
$$

Teorema 1.3.5. Se $P(x, D)$ é um operador elítico em $\Omega$, então $P(x, D)$ é um operador hipoelítico em $\Omega$.

A demonstração deste teorema pode ser encontrada em [9].

Definição 1.3.6. Seja $P(x, D)$ um operador diferencial parcial linear definido no aberto $\Omega \subset \mathbb{R}^{n}$. Definimos o Transposto de $P(x, D)$, como o único operador

$$
{ }^{t} P(x, D): \mathscr{D}^{\prime}(\Omega) \longrightarrow \mathscr{D}^{\prime}(\Omega)
$$

que satisfaz:

Dada $u \in \mathscr{D}^{\prime}(\Omega)$,

$$
\left\langle{ }^{t} P(x, D) u, \varphi\right\rangle \doteq\langle u, P(x, D) \varphi\rangle
$$


para toda $\varphi \in C_{c}^{\infty}(\Omega)$.

Dada $u \in \mathscr{D}^{\prime}(\Omega)$, temos

$$
\begin{gathered}
\left\langle{ }^{t} P(x, D) u, \varphi\right\rangle=\left\langle u, \sum_{|\alpha| \leq m} a_{\alpha}(x) D^{\alpha} \varphi\right\rangle=\sum_{|\alpha| \leq m}\left\langle u, a_{\alpha}(x) D^{\alpha} \varphi\right\rangle \\
=\sum_{|\alpha| \leq m}\left\langle a_{\alpha}(x) u, D^{\alpha} \varphi\right\rangle=\sum_{|\alpha| \leq m}\left\langle(-1)^{|\alpha|} D^{\alpha}\left(a_{\alpha}(x) u\right), \varphi\right\rangle=\left\langle\sum_{|\alpha| \leq m}(-1)^{|\alpha|} D^{\alpha}\left(a_{\alpha}(x) u\right), \varphi\right\rangle,
\end{gathered}
$$

para toda $\varphi \in C_{c}^{\infty}(\Omega)$. Portanto,

$$
{ }^{t} P(x, D)=\sum_{|\alpha| \leq m}(-1)^{|\alpha|} D^{\alpha}\left(a_{\alpha}(x) \cdot\right)
$$

Exemplo 1.3.7. No caso de um ODPL de ordem 1

$$
P(x, D)=\sum_{j=1}^{n} a_{j}(x) D_{x_{j}}+a_{0}(x)
$$

Temos

$$
{ }^{t} P(x, D)=-P(x, D)-\sum_{j=1}^{n} D_{x_{j}}\left(a_{j}\right)(x)+2 a_{0}(x) .
$$

Obs. 1.3.8. Em relação ao símbolo principal, um $O D P \boldsymbol{D}$ é semelhante ao seu transposto. Suponha $P(x, D)=\sum_{|\alpha| \leq m} a_{\alpha}(x) D^{\alpha}$ em $\Omega$. Dado $u \in \mathscr{D}^{\prime}(\Omega)$, por Leibniz

$$
D^{\alpha}\left(a_{\alpha} u\right)=\sum_{\beta \leq \alpha}\left(\begin{array}{l}
\alpha \\
\beta
\end{array}\right) D^{\alpha-\beta}\left(a_{\alpha}\right) D^{\beta}(u)=a_{\alpha} D^{\alpha}(u)+\sum_{\beta<\alpha}\left(\begin{array}{l}
\alpha \\
\beta
\end{array}\right) D^{\alpha-\beta}\left(a_{\alpha}\right) D^{\beta}(u),
$$

para todo $\alpha \in \mathbb{Z}_{+}^{n}$. Segue dai,

$$
{ }^{t} P(x, D)=(-1)^{m} P_{m}(x, D)+\sum_{|\alpha|<m}(-1)^{|\alpha|}\left\{\sum_{\beta \leq \alpha}\left(\begin{array}{l}
\alpha \\
\beta
\end{array}\right) D^{\alpha-\beta}\left(a_{\alpha}\right) D^{\beta}(\cdot)\right\} .
$$

E assim concluímos a seguinte relação entre os símbolos principais de $P$ e $P^{t}$ :

$$
{ }^{t} P_{m}(x, \xi)=(-1)^{m} P_{m}(x, \xi) e m \Omega \times \mathbb{R}^{n} .
$$

Em particular, $P$ é elítico (resp. tipo principal) se, e somente se, ${ }^{t} P$ é elítico (resp. tipo principal). 


\subsection{Alguns resultados de Análise Complexa}

Nesta seção vamos comentar alguns teoremas de Análise Complexa que serão usados neste texto. Também, vamos destacar e discutir o papel do operador de Cauchy-Riemann nesta teoria.

Definição 1.4.1. Seja $f: U \longrightarrow \mathbb{C}$ uma função contínua, onde $U$ é um aberto de $\mathbb{C}$. Dizemos que $f$ é holomorfa (ou analítica) em $U$, se para cada $z_{0} \in U$ existe o limite

$$
f^{\prime}\left(z_{0}\right)=\lim _{h \rightarrow 0} \frac{f\left(z_{0}+h\right)-f\left(z_{0}\right)}{h} .
$$

Se $f$ é holomorfa em $U$ entãa a função obtida acima, $U \ni z \mapsto f^{\prime}(z) \in \mathbb{C}$ é chamada de derivada de $f$ e é denotada por $f^{\prime}$.

Sejam $U, V$ abertos de $\mathbb{C}$ e $f: U \longrightarrow V$ é uma bijeção. Se $f$ é holomorfa em $U$ e $f^{-1}$ é holomorfa em $V$, então $f$ é dita um biholomorfismo de $U$ em $V$.

Exemplo 1.4.2. Polinômios, i.e., $p(z)=a_{n} z^{n}+a_{n-1} z^{n-1}+\cdots+a_{0}, a_{j} \in \mathbb{C}$ com $j \in$ $\{1, \ldots, n\}$ onde $n \in \mathbb{N}, z \in \mathbb{C}$, são exemplos de funções holomorfas em $\mathbb{C}$.

As funções que são holomorfas em todo o plano $\mathbb{C}$ são chamadas de funções inteiras. Os polinômios são funções inteiras.

Exemplo 1.4.3. Outro exemplo de função inteira é a função exponencial

$$
e^{z}=e^{\Re\{z\}}(\cos (\Im\{z\})+i \operatorname{sen}(\Im\{z\})), z \in \mathbb{C} .
$$

Um fato sobre funções holomorfas é que se $f$ é holomorfa, então $f^{\prime}$ é holomorfa. Segue daí que se $f$ é uma função holomorfa em um aberto $U \subset \mathbb{C}$, então $f$ vista como função de duas variáveis reais, i.e.,

$$
\tilde{f}\left(x_{1}, x_{2}\right) \doteq f\left(x_{1}+i x_{2}\right),\left(x_{1}, x_{2}\right) \in\left\{\left(x_{1}, x_{2}\right) \in \mathbb{R}^{2} ; x_{1}+i x_{2} \in U\right\}
$$

é uma função $C^{\infty}$. 


\subsubsection{O Operador de Cauchy-Riemann}

Seja $f$ uma função holomorfa em $U$. Seja $z_{0} \in U, z_{0}=x_{10}+i x_{20}, \operatorname{com} x_{10}, x_{20} \in \mathbb{R}$. Podemos escrever $f(z)=u\left(x_{1}, x_{2}\right)+i v\left(x_{1}, x_{2}\right), z=x_{1}+i x_{2}$, onde $u=\Re\{f\}$ e $v=\Im\{f\}$. Assim, se $h$ é um número real puro, temos

$$
\begin{aligned}
\frac{f\left(z_{0}+h\right)-f\left(z_{0}\right)}{h} & =\frac{\left(u\left(x_{10}+h, x_{20}\right)+i v\left(x_{10}+h, x_{20}\right)\right)-\left(u\left(x_{10}, x_{20}\right)+i v\left(x_{10}, x_{20}\right)\right)}{h} \\
& =\frac{u\left(x_{10}+h, x_{20}\right)-u\left(x_{10}, x_{20}\right)}{h}+i \frac{v\left(x_{10}+h, x_{20}\right)-v\left(x_{10}, x_{20}\right)}{h}
\end{aligned}
$$

Fazendo $h \longrightarrow 0$, temos

$$
f^{\prime}\left(z_{0}\right)=\frac{\partial u}{\partial x_{1}}\left(x_{10}, x_{20}\right)+i \frac{\partial v}{\partial x_{1}}\left(x_{10}, x_{20}\right)
$$

E agora,

$$
\begin{aligned}
\frac{f\left(z_{0}+i h\right)-f\left(z_{0}\right)}{i h} & =\frac{\left(u\left(x_{10}, x_{20}+h\right)+i v\left(x_{10}, x_{20}+h\right)\right)-\left(u\left(x_{10}, x_{20}\right)+i v\left(x_{10}, x_{20}\right)\right)}{i h} \\
& =\frac{u\left(x_{10}, x_{20}+h\right)-u\left(x_{10}, x_{20}\right)}{i h}+i \frac{v\left(x_{10}, x_{20}+h\right)-v\left(x_{10}, x_{20}\right)}{i h} \\
& =-i \frac{u\left(x_{10}, x_{20}+h\right)-u\left(x_{10}, x_{20}\right)}{h}+\frac{v\left(x_{10}, x_{20}+h\right)-v\left(x_{10}, x_{20}\right)}{h}
\end{aligned}
$$

Fazendo $h \longrightarrow 0$, temos

$$
f^{\prime}\left(z_{0}\right)=-i \frac{\partial u}{\partial x_{2}}\left(x_{10}, x_{20}\right)+\frac{\partial v}{\partial x_{2}}\left(x_{10}, x_{20}\right)
$$

Somando (1.4.1) e (1.4.2) obtemos

$$
\begin{aligned}
2 f^{\prime}\left(z_{0}\right) & =\frac{\partial u}{\partial x_{1}}\left(x_{10}, x_{20}\right)+i \frac{\partial v}{\partial x_{1}}\left(x_{10}, x_{20}\right)-i \frac{\partial u}{\partial x_{2}}\left(x_{10}, x_{20}\right)+\frac{\partial v}{\partial x_{2}}\left(x_{10}, x_{20}\right) \\
& =\frac{\partial(u+i v)}{\partial x_{1}}\left(x_{10}, x_{20}\right)-i \frac{\partial(u+i v)}{\partial x_{2}}\left(x_{10}, x_{20}\right)=\frac{\partial f}{\partial x_{1}}\left(x_{10}, x_{20}\right)-i \frac{\partial f}{\partial x_{2}}\left(x_{10}, x_{20}\right) .
\end{aligned}
$$


Logo,

$$
\frac{1}{2}\left(\frac{\partial f}{\partial x_{1}}\left(x_{10}, x_{20}\right)-i \frac{\partial f}{\partial x_{2}}\left(x_{10}, x_{20}\right)\right)=f^{\prime}\left(z_{0}\right)
$$

Por outro lado, subtraindo (1.4.2) de (1.4.1) obtemos

$$
\begin{aligned}
0 & =\frac{\partial u}{\partial x_{1}}\left(x_{10}, x_{20}\right)+i \frac{\partial v}{\partial x_{1}}\left(x_{10}, x_{20}\right)+i \frac{\partial u}{\partial x_{2}}\left(x_{10}, x_{20}\right)-\frac{\partial v}{\partial x_{2}}\left(x_{10}, x_{20}\right) \\
& =\frac{\partial(u+i v)}{\partial x_{1}}\left(x_{10}, x_{20}\right)+i \frac{\partial(u+i v)}{\partial x_{1}}\left(x_{10}, x_{20}\right)
\end{aligned}
$$

Logo

$$
\frac{\partial f}{\partial x_{1}}\left(x_{10}, x_{20}\right)+i \frac{\partial f}{\partial x_{2}}\left(x_{10}, x_{20}\right)=0 .
$$

Assim, no contexto de funções de variáveis complexas surgem naturalmente os operadores

$$
\frac{\partial}{\partial \bar{z}}=\frac{1}{2}\left(\frac{\partial}{\partial x_{1}}+i \frac{\partial}{\partial x_{2}}\right) \text { e } \frac{\partial}{\partial z}=\frac{1}{2}\left(\frac{\partial}{\partial x_{1}}-i \frac{\partial}{\partial x_{2}}\right)
$$

em $\mathbb{R}^{2}$. Eles são chamados de operador de Cauchy-Riemann e anti-operador de CauchyRiemann.

Obs. 1.4.4. Os operadores $\frac{\partial}{\partial \bar{z}} e \frac{\partial}{\partial z}$ são operadores elíticos em $\mathbb{R}^{2}$.

Obs. 1.4.5. O operador $\frac{\partial}{\partial \bar{z}}$ é chamado de operador de Cauchy-Riemann, pelo fato de que encontrar uma solução de sua homogênea, i.e., $\frac{\partial}{\partial \bar{z}}(f)=0$, equivale a obter a solução do sistema

$$
\left\{\begin{aligned}
\frac{\partial u}{\partial x_{1}} & =\frac{\partial v}{\partial x_{2}} \\
\frac{\partial u}{\partial x_{2}} & =-\frac{\partial v}{\partial x_{1}}
\end{aligned}\right.
$$

onde $u, v$ são as partes real e imaginária de $f$, respectivamente. O sistema é chamado de equações de Cauchy-Riemann e recebem este nome em homenagem ao matemático francês A. L. Cauchy, que as descobriu e usou, e ao matemático alemão G. F. B. Riemann, que as tornou fundamentais na teoria das funções holomorfas.

Teorema 1.4.6. Seja $f \in \mathscr{D}^{\prime}(U), U \subset \mathbb{R}^{2}$ um aberto, tal que $\frac{\partial}{\partial \bar{z}}(f)=0$ em $U$. Então $f$, na variável $z=x_{1}+i x_{2}$, é uma função holomorfa no aberto $\{z \in \mathbb{C} ;(\Re\{z\}, \Im\{z\}) \in U\}$. 
Demonstração: Um teorema conhecido sobre as equações de Cauchy-Riemann é o seguinte:

Sejam u e $v$ funções reais de classe $C^{1}$ em aberto $V$ de $\mathbb{R}^{2}$, nas variáveis $x_{1}$ e $x_{2}$. Se suas derivadas parciais satisfazem às equações de Cauchy-Riemann em V, i.e.,

$$
\frac{\partial u}{\partial x_{1}}=\frac{\partial v}{\partial x_{2}} \quad e \frac{\partial u}{\partial x_{2}}=-\frac{\partial v}{\partial x_{1}}
$$

em V. Então a função

$$
f(z) \doteq u\left(x_{1}, x_{2}\right)+i v\left(x_{1}, x_{2}\right), z=x_{1}+i x_{2},
$$

é uma função holomorfa em $\left\{x_{1}+i x_{2} ;\left(x_{1}, x_{2}\right) \in V\right\}$.

Da observação 1.4.4 e do teorema 1.3.5 temos que $\frac{\partial}{\partial \bar{z}}$ é hipoelítico. Daí, como $\frac{\partial}{\partial \bar{z}}(f)=$ 0 em $U$, segue que $f \in C^{\infty}(U)$. Além disso, como foi comentado na observação 1.4.5, segue que as partes real e imaginária da função $f$ satisfazem as condições de CauchyRiemann em $U$. Do teorema comentado acima temos que $f$, na variável $z=x_{1}+i x_{2}$, é uma função holomorfa no aberto $\{z \in \mathbb{C} ;(\Re\{z\}, \Im\{z\}) \in U\}$.

\subsubsection{Alguns teoremas}

Teorema 1.4.7. Seja $f(z)$ holomorfa no aberto $U \subset \mathbb{C}$. Dado $z_{0} \in U$, seja $r_{0}>0$ tal que, $B_{r_{0}}\left(z_{0}\right)$, a bola de centro $z_{0}$ e raio $r_{0}>0$ esteja contida em $U$. Então, em cada ponto $z \in B_{r_{0}}\left(z_{0}\right)$ tem-se

$$
f(z)=\sum_{j=0}^{\infty} \frac{f^{(j)}\left(z_{0}\right)}{j !}\left(z-z_{0}\right)^{j}
$$

i.e., a série infinita acima convege para $f(z)$.

Quando $f$ é uma função inteira, o raio de convergência é infinito, i.e., dado $z_{0} \in \mathbb{C}$, temos

$$
f(z)=\sum_{j=0}^{\infty} \frac{f^{(j)}\left(z_{0}\right)}{j !}\left(z-z_{0}\right)^{j} \text { para todo } z \in \mathbb{C} .
$$


Teorema 1.4.8. Se $f$ é uma função holomorfa num aberto conexo $U \subset \mathbb{C}$, então $f$ é aberta ou constante.

Teorema 1.4.9 (Teorema de Liouville). Uma função inteira limitada é constante.

Segue deste teorema que o plano complexo $\mathbb{C}$ e o disco aberto de raio 1 e centrado na origem $\mathbb{D}$ não são biholomorfos.

\subsubsection{Convergência de sequências de funções holomorfas}

Como vimos no teorema 1.4.7, uma função holomorfa, pode ser aproximada localmente por polinômios, que também são funções holomorfas. O que pode ser dito sobre uma possível recíproca da frase anterior? Nesta seção vamos estabelecer um critério de convergência de sequência de funções, em particular de funções holomorfas, e apresentar um teorema (1.4.11) que terá grande importância no estudo da estrutura local das soluções de um campo hipoelítico.

Definição 1.4.10. Seja $U \subset \mathbb{C}$ um aberto. Dizemos que uma sequência de funções $\left(f_{j}\right)_{j \in \mathbb{N}}$ em $U$ converge uniformemente em compactos de $U$ se existe uma função $f$ definida em $U$, tal que a sequência de restrições $\left(\left.f_{j}\right|_{K}\right)_{j \in \mathbb{N}}$ converge uniformemente para $\left.f\right|_{K}$ para todo compacto $K \subset U$.

É um fato conhecido da Análise que se uma sequência de funções contínuas converge uniformemente para uma função $f$, então $f$ é contínua. Segue daí, que se $\left(f_{j}\right)_{j \in \mathbb{N}}$ é uma sequência funções em $U$, que são contínuas, e que converge uniformemente em compactos de $U$ para $f$, então $f$ é contínua em $U$. Como fica o caso da sequência em questão ser uma sequência de funções holomorfas? O próximo teorema trata deste caso.

Teorema 1.4.11. Sejam $U \subset \mathbb{C}$ um aberto $e\left(f_{j}\right)_{j \in \mathbb{N}}$ uma sequência de funções holomorfas em $U$ que converge uniformente em compactos de $U$ para a função $f$. Então $f$ é holomorfa em $U$.

Este teorema pode ser encarado como uma consequência direta dos teoremas de Morera e de Goursat. A demonstração deste teorema pode ser encontrada em [22]. 


\subsection{Campos Vetoriais Complexos}

Nesta seção vamos introduzir o conceito de campos vetoriais complexos. A maioria das definições e resultados deste capítulo pode ser generalizada para o contexto de variedades diferenciáveis. O leitor interessado pode encontrar em [5] o assunto tratado numa forma mais geral.

\subsubsection{Campos Vetoriais Complexos}

Definição 1.5.1. Um campo vetorial complexo em um aberto $\Omega$ de $\mathbb{R}^{2}$ é uma aplicação $\mathbb{C}$-linear

$$
L: C^{\infty}(\Omega) \longrightarrow C^{\infty}(\Omega)
$$

que satisfaz a regra de Leibniz

$$
L(f g)=L(f) g+f L(g), \quad f, g \in C^{\infty}(\Omega) .
$$

Denotamos por $\mathfrak{X}(\Omega)$ o conjunto dos campos definidos em $\Omega$.

Exemplo 1.5.2. Para cada $1 \leq j \leq 2$ a aplicação de $C^{\infty}(\Omega) \longrightarrow C^{\infty}(\Omega)$

$$
f \longmapsto \frac{\partial f}{\partial x_{j}}
$$

que leva $f$ em sua $j$-ésima derivada parcial, define um elemento de $\mathfrak{X}(\Omega)$. Mais ainda, se $a_{j} \in C^{\infty}(\Omega)$ para $1 \leq j \leq 2$, então a aplicação de $C^{\infty}(\Omega) \longrightarrow C^{\infty}(\Omega)$

$$
f \longmapsto a_{1} \frac{\partial f}{\partial x_{1}}+a_{2} \frac{\partial f}{\partial x_{2}}
$$

define um elemento de $\mathfrak{X}(\Omega)$. Em outras palavras, um operador diferencial parcial linear de ordem 1 sem termo constante define um campo vetorial complexo em $\Omega$.

Proposição 1.5.3. Se $L \in \mathfrak{X}(\Omega)$ e $f \in C^{\infty}(\Omega)$ é uma função constante, então $L(f)=0$.

Demonstração: A demonstração é consequência direta de $L$ ser $\mathbb{C}$-linear e satisfazer a regra de Leibniz.

Se $f \equiv c$ em $\Omega$, onde $c \in \mathbb{C}$ é uma constante, então 


$$
L(f)=L(c)=L(c \cdot 1)=c L(1) .
$$

Por outro lado

$$
L(1)=L\left(1^{2}\right)=L(1) \cdot 1+1 \cdot L(1)=2 L(1)
$$

$\operatorname{logo} L(1)=0$. Portanto $L(f)=0$.

Proposição 1.5.4. Se $L \in \mathfrak{X}(\Omega)$ então

$$
\operatorname{supp} L(f) \subset \operatorname{supp} f,
$$

para toda $f \in C^{\infty}(\Omega)$.

\section{Demonstração:}

Vamos mostrar que se $f \in C^{\infty}(\Omega)$, é tal que $f=0$ em um aberto $V \subset \Omega$, então $L(f)=0$ em $V$.

Seja $p \in V$. Escolha $\varphi \in C_{c}^{\infty}(\Omega)$ tal que $\operatorname{supp}(\varphi) \subset V$ e $\varphi(p)=1$. Note que

$$
f=(1-\varphi) f \text { em } \Omega
$$

De fato, se $q \in V$, temos que $f(q)=0$ e $(1-\varphi(q)) f(q)=0$. Agora, se $q \notin V$, então $\varphi(q)=0$. Logo, $(1-\varphi(q)) f(q)=(1-0) f(q)=f(q)$.

Assim,

$$
\begin{aligned}
L(f) & =L([1-\varphi] f) \\
& =(1-\varphi) L(f)+f L(1-\varphi)
\end{aligned}
$$

Logo,

$$
L(f)(p)=(1-\underbrace{\varphi(p)}_{=1}) L(f)(p)+\underbrace{f(p)}_{=0} L(1-\varphi)(p)=0
$$

Ou seja, 


$$
L(f)(p)=0
$$

Como $p \in V$ foi tomado arbitrariamente, temos que $L(f)=0$ em $V$.

Obs. 1.5.5. Dados $g \in C^{\infty}(\Omega)$ e $L \in \mathfrak{X}(\Omega)$ definimos $g L \in \mathfrak{X}(\Omega)$ por

$$
(g L)(f)=g \cdot L(f), f \in C^{\infty}(\Omega) .
$$

Esse produto torna $\mathfrak{X}(\Omega)$ um $C^{\infty}(\Omega)$-módulo.

Uma consequência da proposição 1.5.4 é a possibilidade de definir a restrição de um elemento $L \in \mathfrak{X}(\Omega)$ ao um subconjunto aberto $U$ de $\Omega$. Mais precisamente:

Corolário 1.5.6. Para cada $L \in \mathfrak{X}(\Omega)$, existe $\left.L\right|_{U} \in \mathfrak{X}(U)$, tal que o diagrama

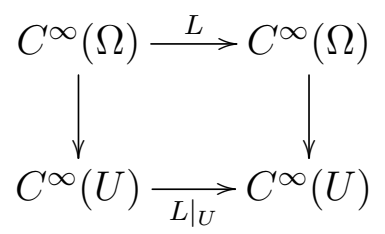

é comutativo.

As setas verticais representam a aplicação restrição: $\left.C^{\infty}(\Omega) \ni f \mapsto f\right|_{U} \in C^{\infty}(U)$.

\section{Demonstração:}

Defina

$$
\left.L\right|_{U}(f)(p)=L(\tilde{f})(p), p \in U, f \in C^{\infty}(U)
$$

Onde $\tilde{f}$ é qualquer elemento em $C^{\infty}(\Omega)$ que coincide com $f$ em uma vizinhança de $p$.

Vejamos que $\left.L\right|_{U}$ está bem definido. Dado $f \in C^{\infty}(U)$ e $p \in U$, $\operatorname{sejam~} \tilde{f}, \tilde{g} \in C^{\infty}(\Omega)$ tais que $\tilde{f}=f$ em $V_{1}$ e $\tilde{g}=f$ em $V_{2}$, com $V_{1}, V_{2}$ vizinhanças de $p$. Seja $V=V_{1} \cap V_{2}$. Temos que $p \in V$ e $\tilde{f}=\tilde{g}$ em $V$. Logo $\tilde{f}-\tilde{g}=0$ em $V$. Pela proposição 1.5.4 temos $L(\tilde{f}-\tilde{g})=0$ em $V$. Logo $L(\tilde{f})=L(\tilde{g})$ em $V$. E assim $L(\tilde{f})(p)=L(\tilde{g})(p)$. É fácil ver que $\left.L\right|_{U}(f) \in C^{\infty}(U)$, para toda $f \in C^{\infty}(U)$. Com uma simples verificação concluimos que 
$\left.L\right|_{U}$ é $\mathbb{C}$-linear e satisfaz a regra de Leibniz.

Agora observe que $\left.(L(f))\right|_{U}=\left.L\right|_{U}\left(\left.f\right|_{U}\right)$. De fato, se $f \in C^{\infty}(\Omega)$, temos que $\left.f\right|_{U} \in$ $C^{\infty}(U)$. Assim $\left.L\right|_{U}\left(\left.f\right|_{U}\right)(p)=L(f)(p)$, para todo $p \in U$ (escolhemos $\left.f \tilde{\mid}_{U}=f\right)$. Ou seja, o diagrama acima é comutativo.

Obs. 1.5.7. Como de costume, para não sobrecarregar a notação, escrevemos L ao invés de $\left.L\right|_{U}$.

O corolário acima será importante no desenvolvimento da demonstração do próximo teorema. Ele diz que essencialmente os campos descritos no exemplo 1.5.2 são os únicos elementos de $\mathfrak{X}(\Omega)$. Mas antes, vamos fixar uma notação. Considere

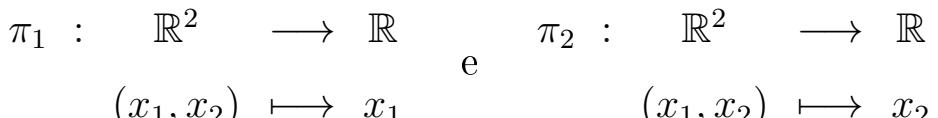

como as projeções canônicas do $\mathbb{R}^{2}$. Note que $\pi_{1}, \pi_{2} \in C^{\infty}\left(\mathbb{R}^{2}\right)$.

Teorema 1.5.8. Seja $L \in \mathfrak{X}(\Omega)$, onde $\Omega$ é um subconjunto aberto do $\mathbb{R}^{2}$. Então

$$
L=L\left(\pi_{1}\right) \frac{\partial}{\partial x_{1}}+L\left(\pi_{2}\right) \frac{\partial}{\partial x_{2}} .
$$

Outra notação comum para isto é

$$
L=L x_{1} \frac{\partial}{\partial x_{1}}+L x_{2} \frac{\partial}{\partial x_{2}} .
$$

Na demonstração do teorema acima vamos usar o seguinte lema:

Lema 1.5.9. Seja $f: U \longrightarrow \mathbb{C}$ uma função de classe $C^{\infty}$, onde $U \subset \Omega \subset \mathbb{R}^{2}$ é um aberto convexo contendo p. Então, existem funções $g_{1}, g_{2}: U \longrightarrow \mathbb{C}$ de classe $C^{\infty}$, tais que:

$$
f(x)=f(p)+\pi_{1}(x-p) g_{1}(x)+\pi_{2}(x-p) g_{2}(x),
$$

para todo $x \in U$. 
Seja $p=\left(p_{1}, p_{2}\right) \in \Omega$. Como $\Omega$ é aberto existe uma bola $B(p)$ com centro em $p$ tal que $B(p) \subset \Omega$. Dado $f \in C^{\infty}(\Omega)$, pelo lema precedente, podemos escrever

$$
f(x)=f(p)+\pi_{1}(x) g_{1}(x)-\pi_{1}(p) g_{1}(x)+\pi_{2}(x) g_{2}(x)-\pi_{2}(p) g_{2}(x),
$$

para todo $x \in B(p)$, onde $g_{1}, g_{2} \in C^{\infty}(B(p))$. Assim

$$
\begin{aligned}
L(f)(x)=L( & \left.\pi_{1}(x) g_{1}(x)\right)-\pi_{1}(p) L\left(g_{1}\right)(x)+L\left(\pi_{2}(x) g_{2}(x)\right)-\pi_{2}(p) L\left(g_{2}\right)(x)= \\
= & L\left(\pi_{1}\right)(x) g_{1}(x)+\pi_{1}(x) L\left(g_{1}\right)(x)-\pi_{1}(p) L\left(g_{1}\right)(x) \\
& +L\left(\pi_{2}\right)(x) g_{2}(x)+\pi_{2}(x) L\left(g_{2}\right)(x)-\pi_{2}(p) L\left(g_{2}\right)(x)
\end{aligned}
$$

Logo,

$$
L(f)(p)=L\left(\pi_{1}\right)(p) g_{1}(p)+L\left(\pi_{2}\right)(p) g_{2}(p)
$$

Agora observe

$$
\begin{aligned}
\frac{\partial f}{\partial x_{1}}(p) & =\lim _{t \rightarrow 0} \frac{f\left(p_{1}+t, p_{2}\right)-f\left(p_{1}, p_{2}\right)}{t} \\
& =\lim _{t \rightarrow 0} \frac{\pi_{1}((t, 0)) g_{1}\left(p_{1}+t, p_{2}\right)+\pi_{2}((t, 0)) g_{2}\left(p_{1}+t, p_{2}\right)}{t} \\
& =\lim _{t \rightarrow 0} \frac{t g_{1}\left(p_{1}+t, p_{2}\right)}{t} \\
& =\lim _{t \rightarrow 0} g_{1}\left(p_{1}+t, p_{2}\right) \\
& =g_{1}(p) .
\end{aligned}
$$

Analogamente obtemos

$$
\frac{\partial f}{\partial x_{2}}(p)=g_{2}(p)
$$

Logo,

$$
L(f)(p)=L\left(\pi_{1}\right)(p) \frac{\partial f}{\partial x_{1}}(p)+L\left(\pi_{2}\right)(p) \frac{\partial f}{\partial x_{2}}(p) .
$$


Como $p$ é um ponto arbitrário de $\Omega$, temos

$$
L(f)=L\left(\pi_{1}\right) \frac{\partial f}{\partial x_{1}}+L\left(\pi_{2}\right) \frac{\partial f}{\partial x_{2}} \text { em } \Omega .
$$

E como $f \in C^{\infty}(\Omega)$ também foi tomada de forma arbitrária, temos

$$
L=L\left(\pi_{1}\right) \frac{\partial}{\partial x_{1}}+L\left(\pi_{2}\right) \frac{\partial}{\partial x_{2}} \text { em } \Omega
$$

Obs. 1.5.10. O teorema acima tem uma versão no caso mais geral, em que $\Omega$ é um aberto de $\mathbb{R}^{n}$, e com demonstração análoga à que foi feita acima.

Definição 1.5.11. Dizemos que um campo vetorial complexo $L$ em $\Omega, \Omega \subset \mathbb{R}^{2}$ um aberto, é singular em um ponto $p \in \Omega$, se

$$
L\left(\pi_{1}\right)(p)=L\left(\pi_{2}\right)(p)=0 .
$$

Dizemos que L é não singular em $\Omega$ se $L$ é não singular em todo ponto de $\Omega$, equivalentemente,

$$
\left|L\left(\pi_{1}\right)\right|+\left|L\left(\pi_{2}\right)\right| \neq 0 \text { em } \Omega .
$$

Se $L$ é um campo vetorial complexo definido no aberto $\Omega \subset \mathbb{R}^{2}$, pelo teorema acima podemos escrever

$$
L=i L\left(\pi_{1}\right) D_{x_{1}}+i L\left(\pi_{2}\right) D_{x_{2}}
$$

em $\Omega$. Ou seja, $L$ pode ser escrito com um operador diferencial parcial linear de ordem 1 sem termos de ordem 0 . Em particular, podemos estender $L$ a $\mathscr{D}^{\prime}(\Omega)$.

Definição 1.5.12. Definimos $L(x, D) \doteq i L\left(\pi_{1}\right) D_{x_{1}}+i L\left(\pi_{1}\right) D_{x_{2}}$ em $\Omega$, e é chamado de o operador diferencial parcial linear que representa $L$ em $\Omega$.

Note que, dizer que $L$ é um campo não singular, equivale a dizer que o operador diferencial parcial linear que representa $L$ em $\Omega, L(x, D)$, é de tipo principal. De fato, $L$ é não singular se, e somente se, $\left|L\left(\pi_{1}\right)\right|+\left|L\left(\pi_{2}\right)\right| \neq 0$ em $\Omega$, que é equivalente a $d_{\xi} P_{1}(x, \xi)=i L\left(\pi_{1}\right) d \xi_{1}+i L\left(\pi_{2}\right) d \xi_{2} \neq 0$ em $\Omega$. Dizemos que $L$ é hipoelítico em $\Omega$ se $L(x, D)$ é hipoelítico em $\Omega$. Analogamente, $L$ é elítico em $\Omega$ se $L(x, D)$ é elítico em $\Omega$. 


\subsubsection{Formas Diferenciais}

Nesta seção vamos falar um pouco sobre 1-formas complexas e enunciar alguns resultados que serão usados neste trabalho. Em [5] encontramos um estudo detalhado sobre este tema.

Definição 1.5.13. Uma 1-forma complexa em $\Omega$ é uma aplicação $C^{\infty}(\Omega)$-linear

$$
\omega: \mathfrak{X}(\Omega) \longrightarrow C^{\infty}(\Omega) .
$$

Em outras palavras, o conjunto das 1-formas complexas em $\Omega$, denotado por $\mathcal{N}(\Omega)$, é o dual do $C^{\infty}(\Omega)$-módulo $\mathfrak{X}(\Omega)$, onde $\mathfrak{X}(\Omega)$ é o conjunto dos campos vetoriais complexos definidos em $\Omega$.

Exemplo 1.5.14. Seja $\Omega \subset \mathbb{R}^{2}$ um aberto. Um exemplo simples de 1-forma complexa é dado por

$$
\begin{aligned}
d x_{j}: \mathfrak{X}(\Omega) & \longrightarrow C^{\infty}(\Omega) \\
L & \longmapsto L\left(\pi_{j}\right)
\end{aligned}
$$

$j=1,2$.

Exemplo 1.5.15. Nas mesmas condições do exemplo acima, temos que se $A, B \in C^{\infty}(\Omega)$, então

$$
\omega=A d x_{1}+B d x_{2}
$$

é uma 1-forma complexa em $\Omega$.

Essencialmente, essas são as únicas 1-formas complexas definidas em $\Omega$. De fato, seja $\omega \in \mathcal{N}(\Omega)$. Dado $L \in \mathfrak{X}(\Omega)$, pelo teorema 1.5.8 podemos escrever

$$
L=L\left(\pi_{1}\right) \frac{\partial}{\partial x_{1}}+L\left(\pi_{2}\right) \frac{\partial}{\partial x_{2}}
$$

em $\Omega$. Assim,

$$
\begin{aligned}
\omega(L) & =\omega\left(L\left(\pi_{1}\right) \frac{\partial}{\partial x_{1}}+L\left(\pi_{2}\right) \frac{\partial}{\partial x_{2}}\right)=\omega\left(L\left(\pi_{1}\right) \frac{\partial}{\partial x_{1}}\right)+\omega\left(L\left(\pi_{2}\right) \frac{\partial}{\partial x_{2}}\right) \\
& =L\left(\pi_{1}\right) \omega\left(\frac{\partial}{\partial x_{1}}\right)+L\left(\pi_{2}\right) \omega\left(\frac{\partial}{\partial x_{2}}\right)=\omega\left(\frac{\partial}{\partial x_{1}}\right) d x_{1}(L)+\omega\left(\frac{\partial}{\partial x_{2}}\right) d x_{2}(L) .
\end{aligned}
$$


Pela arbitrariedade de $L \in \mathfrak{X}(\Omega)$, temos

$$
\omega=A d x_{1}+B d x_{2}
$$

em $\Omega$, onde $A, B \in C^{\infty}(\Omega)$ são dadas por $A=\omega\left(\frac{\partial}{\partial x_{1}}\right)$ e $B=\omega\left(\frac{\partial}{\partial x_{2}}\right)$.

Definição 1.5.16. Dada $f \in C^{\infty}(\Omega)$, definimos $d f \in \mathcal{N}(\Omega)$ pela fórmula

$$
d f(L)=L(f), L \in \mathfrak{X}(\Omega) .
$$

Dada $f \in C^{\infty}(\Omega)$ sempre podemos obter um campo $L \in \mathfrak{X}(\Omega)$ tal que $d f(L)=0$. De fato, basta tomar o campo

$$
H_{f}=\frac{\partial f}{\partial x_{2}} \frac{\partial}{\partial x_{1}}-\frac{\partial f}{\partial x_{1}} \frac{\partial}{\partial x_{2}}
$$

em $\Omega$. Temos

$$
d f\left(H_{f}\right)=H_{f}(f)=\frac{\partial f}{\partial x_{2}} \frac{\partial f}{\partial x_{1}}-\frac{\partial f}{\partial x_{1}} \frac{\partial f}{\partial x_{2}}=0 .
$$

Mais ainda, dada uma 1-forma complexa $\omega \in \mathcal{N}(\Omega)$, sempre podemos obter um campo $L$ tal que $\omega(L)=0$. De fato, basta tomar

$$
L=\omega\left(\frac{\partial}{\partial x_{2}}\right) \frac{\partial}{\partial x_{1}}-\omega\left(\frac{\partial}{\partial x_{1}}\right) \frac{\partial}{\partial x_{2}}
$$

em $\Omega$. Temos

$$
\omega(L)=\omega\left(\omega\left(\frac{\partial}{\partial x_{2}}\right) \frac{\partial}{\partial x_{1}}-\omega\left(\frac{\partial}{\partial x_{1}}\right) \frac{\partial}{\partial x_{2}}\right)=\omega\left(\frac{\partial}{\partial x_{2}}\right) \omega\left(\frac{\partial}{\partial x_{1}}\right)-\omega\left(\frac{\partial}{\partial x_{1}}\right) \omega\left(\frac{\partial}{\partial x_{2}}\right)=0 .
$$

Definição 1.5.17. Seja $\omega$ uma 1-forma complexa definida em um aberto $U$ de $\mathbb{R}^{2}$. Dizemos que $\omega$ é exata em $U$ se existe uma função $f: U \longrightarrow \mathbb{C}$ tal que $d f=\omega$.

Teorema 1.5.18. Seja $\omega=A d x_{1}+B d x_{2}$ uma 1-forma complexa definida em um aberto convexo $U$ de $\mathbb{R}^{2}$, tal que

$$
\frac{\partial A}{\partial x_{2}}=\frac{\partial B}{\partial x_{1}} e m U
$$

Então, $\omega$ é exata.

Proposição 1.5.19. Seja $L \in \mathfrak{X}(\Omega)$ um campo não singular e $Z \in C^{\infty}(\Omega)$, com $d Z \neq 0$, tais que $L Z=0$ em $\Omega$. Então existe $\lambda_{Z} \in C^{\infty}(\Omega)$, com $\lambda_{Z}(p) \neq 0$ para todo $p \in \Omega$, tal que 


$$
L=\lambda_{Z}\left(\frac{\partial Z}{\partial x_{2}} \frac{\partial}{\partial x_{1}}-\frac{\partial Z}{\partial x_{1}} \frac{\partial}{\partial x_{2}}\right)
$$

em $\Omega$.

\section{Demonstração:}

Como $d Z \neq 0$ temos que $\operatorname{Ker}(d Z)=\{M \in \mathfrak{X}(\Omega) ; d Z(M)=0\}$ tem dimensão 1, i.e., se $N \in \operatorname{Ker}(d Z)$ é um campo não singular, podemos escrever $\operatorname{Ker}(d Z)=\left\{\lambda N ; \lambda \in C^{\infty}(\Omega)\right\}$. Novamente, como $d Z \neq 0$ segue que $\left|\partial_{x_{1}} Z\right|+\left|\partial_{x_{2}} Z\right| \neq 0$ em $\Omega$, daí segue que $H_{Z}$ é um campo não singular em $\Omega$. Note também que $L, H_{Z} \in \operatorname{Ker}(d Z)$. Logo existe $\lambda_{Z} \in C^{\infty}(\Omega)$ tal que

$$
L=\lambda_{Z} H_{Z}
$$

em $\Omega$. Como $L$ e $H_{Z}$ são não singulares em $\Omega$, segue que $\lambda_{Z}$ não se anula em nenhum ponto de $\Omega$. Assim, temos o resultado.

Agora vamos fazer um breve e simples comentário sobre integração de 1-formas complexas sobre curvas. O leitor poderá encontrar um estudo detalhado sobre este assunto em [22] e [18].

Seja $\omega \in \mathcal{N}(\Omega)$ uma 1-forma complexa. Suponha $\omega$ escrita da forma

$$
\omega=A d x_{1}+B d x_{2}
$$

em $\Omega$, onde $A, B \in C^{\infty}(\Omega)$. Seja $\gamma:[a, b] \longrightarrow \Omega$ uma curva $C^{\infty}$, i.e., uma função $C^{\infty} \mathrm{em}$ $(a, b)$ que possui uma extensão $C^{\infty}$ a um aberto que contenha $[a, b]$. Definimos a integral de $\omega$ em $\alpha$ como sendo o número complexo

$$
\int_{\gamma} \omega=\int_{a}^{b}\left[A(\gamma(s)) \gamma_{1}^{\prime}(s)+B(\gamma(s)) \gamma_{2}^{\prime}(s)\right] d s .
$$

Onde $\gamma_{1}, \gamma_{2}$ representam a primeira e segunda coordenadas de $\gamma$ respectivamente. No cálculo da integral acima subentende-se que, se $A(\gamma(s)) \gamma_{1}^{\prime}(s)+B(\gamma(s)) \gamma_{2}^{\prime}(s)=x(s)+i t(s)$, onde $x(s)$ e $t(s)$ são as partes real e imaginária da expressão, então 


$$
\int_{\gamma} \omega=\int_{a}^{b}(x(s)+i t(s)) d s=\int_{a}^{b} x(s) d s+i \int_{a}^{b} t(s) d s .
$$

Um teorema interessante relacionado com integração de 1-formas complexas é o teorema de Green. Por simplificação vamos apenas enunciar um caso particular deste teorema, no entanto, este caso particular é suficiente para este trabalho.

Teorema 1.5.20. Seja $U \subset \mathbb{R}^{2}$ um aberto e $\omega=A d x_{1}+B d x_{2}$ uma 1-forma complexa definda em $U$. Suponha que $W \subset U$ é um aberto, tal que $\bar{W} \subset U$ é um compacto, mais ainda, $\bar{W}$ é um polígono. Suponha também que a fronteira de $\bar{W}$, que é uma curva poligonal fechada, que denotaremos por $\gamma$, está orientada positivamente e é formada pela justaposiçãa das curvas $\gamma_{j}, j \in\{1, \ldots, n\}$, que são segmentos retilíneos, i.e., $\gamma=\gamma_{1} * \gamma_{2} *$ $\cdots * \gamma_{n}$. Então

$$
\int_{W}\left(\frac{\partial B}{\partial x_{1}}-\frac{\partial A}{\partial x_{2}}\right) d x_{1} d x_{2}=\sum_{j=1}^{n} \int_{\gamma_{j}} \omega=\int_{\gamma} \omega .
$$

Obs. 1.5.21. Seja $\gamma:[a, b] \longrightarrow \mathbb{R}^{2}$ uma curva fechada sem auto-interseções e $U$ a região delimitada por $\gamma$. Grosso modo, dizemos que $\gamma$ possui orientação positiva quando o parâmetro s cresce de a até b deixando $U$ do lado esquerdo de $\gamma$.

\subsubsection{Mudança de Variáveis em Campos}

Sejam $\Omega_{1}, \Omega_{2}$ abertos do $\mathbb{R}^{2}$ e denotaremos por $\left(x_{1}, x_{2}\right)$ um elemento genérico de $\Omega_{1}$, e por $\left(y_{1}, y_{2}\right)$ um elemento genérico de $\Omega_{2}$.

Seja $L: C^{\infty}\left(\Omega_{1}\right) \longrightarrow C^{\infty}\left(\Omega_{1}\right)$ um campo em $\Omega_{1}$ e $F: \Omega_{1} \longrightarrow \Omega_{2}$ um difeomorfismo $C^{\infty}$

Dado $f \in C^{\infty}\left(\Omega_{2}\right)$, temos que $F^{*}(f)=f \circ F \in C^{\infty}\left(\Omega_{1}\right)$. Aplicando $L$ em $F^{*}(f)$ :

$$
\begin{gathered}
L(f \circ F)(x)=L\left(\pi_{1}\right)(x) \frac{\partial(f \circ F)}{\partial x_{1}}(x)+L\left(\pi_{2}\right)(x) \frac{\partial(f \circ F)}{\partial x_{2}}(x)= \\
\quad=L\left(\pi_{1}\right)(x)\left[\frac{\partial f}{\partial y_{1}}(F(x)) \frac{\partial F_{1}}{\partial x_{1}}(x)+\frac{\partial f}{\partial y_{2}}(F(x)) \frac{\partial F_{2}}{\partial x_{1}}(x)\right] \\
+L\left(\pi_{2}\right)(x)\left[\frac{\partial f}{\partial y_{1}}(F(x)) \frac{\partial F_{1}}{\partial x_{2}}(x)+\frac{\partial f}{\partial y_{2}}(F(x)) \frac{\partial F_{2}}{\partial x_{2}}(x)\right]=
\end{gathered}
$$




$$
\begin{gathered}
=\left[L\left(\pi_{1}\right)(x) \frac{\partial F_{1}}{\partial x_{1}}(x)+L\left(\pi_{2}\right)(x) \frac{\partial F_{1}}{\partial x_{2}}(x)\right] \frac{\partial f}{\partial y_{1}}(F(x)) \\
+\left[L\left(\pi_{1}\right)(x) \frac{\partial F_{2}}{\partial x_{1}}(x)+L\left(\pi_{2}\right)(x) \frac{\partial F_{2}}{\partial x_{2}}(x)\right] \frac{\partial f}{\partial y_{2}}(F(x)) \\
=L\left(F_{1}\right)(x) \frac{\partial f}{\partial y_{1}}(F(x))+L\left(F_{2}\right)(x) \frac{\partial f}{\partial y_{2}}(F(x)),
\end{gathered}
$$

para $x \in \Omega_{1}$. Ou seja, dada $f \in C^{\infty}\left(\Omega_{2}\right)$ temos

$$
L(f \circ F)(x)=L\left(\pi_{1} \circ F\right)(x) \frac{\partial f}{\partial y_{1}}(F(x))+L\left(\pi_{2} \circ F\right)(x) \frac{\partial f}{\partial y_{2}}(F(x)),
$$

para $x \in \Omega_{1}$. Daí,

$$
\left(L(f \circ F) \circ F^{-1}\right)(y)=\left(L\left(F_{1}\right) \circ F^{-1}\right)(y) \frac{\partial f}{\partial y_{1}}(y)+\left(L\left(F_{2}\right) \circ F^{-1}\right)(y) \frac{\partial f}{\partial y_{2}}(y),
$$

para $y \in \Omega_{2}$.

Da conta acima, segue a definição

$$
L_{F}(u)=\left(F^{-1}\right)^{*}\left(L\left(F^{*}(u)\right)\right),
$$

para $u \in \mathscr{D}^{\prime}\left(\Omega_{2}\right)$. Pelo teorema 1.2.31 $L_{F}$ está bem definido e, por definição, temos que $L_{F}$ é um operador linear de $\mathscr{D}^{\prime}\left(\Omega_{2}\right)$ em $\mathscr{D}^{\prime}\left(\Omega_{2}\right)$.

Proposição 1.5.22. $L_{F}$ é um campo em $\Omega_{2}$. Isto é, a restrição de $L_{F}$ a $C^{\infty}\left(\Omega_{2}\right)$ é um campo em $\Omega_{2}$.

\section{Demonstração:}

Pela definição de $L_{F}$ e pelo teorema 1.2.31 é fácil ver que se $f \in C^{\infty}\left(\Omega_{2}\right)$, então $L_{F}(f) \in$ $C^{\infty}\left(\Omega_{2}\right)$. Também temos que $L_{F}$ é linear, pois é composição de aplicações lineares. Sejam $f, g \in C^{\infty}\left(\Omega_{2}\right)$. Então

$$
\begin{gathered}
L_{F}(f g)=\left(F^{-1}\right)^{*}\left(L\left(F^{*}(f g)\right)\right)=\left(F^{-1}\right)^{*}\left(L\left(F^{*}(f) F^{*}(g)\right)\right) \\
=\left(F^{-1}\right)^{*}\left(L\left(F^{*}(f)\right) F^{*}(g)+F^{*}(f) L\left(F^{*}(g)\right)\right) \\
=\left(F^{-1}\right)^{*}\left(L\left(F^{*}(f)\right) F^{*}(g)\right)+\left(F^{-1}\right)^{*}\left(F^{*}(f) L\left(F^{*}(g)\right)\right)
\end{gathered}
$$




$$
\begin{gathered}
=\left(F^{-1}\right)^{*}\left(L\left(F^{*}(f)\right)\right)\left(F^{-1}\right)^{*}\left(F^{*}(g)\right)+\left(F^{-1}\right)^{*}\left(F^{*}(f)\right)\left(F^{-1}\right)^{*}\left(L\left(F^{*}(g)\right)\right) \\
=\left(F^{-1}\right)^{*}\left(L\left(F^{*}(f)\right)\right) g+f\left(F^{-1}\right)^{*}\left(L\left(F^{*}(g)\right)\right) \\
=L_{F}(f) g+f L_{F}(g) .
\end{gathered}
$$

Portanto vale Leibniz. Assim, a proposição está demonstrada.

Assim fica bem definida a aplicação

$$
\begin{gathered}
\mathfrak{X}\left(\Omega_{1}\right) \quad \longrightarrow \mathfrak{X}\left(\Omega_{2}\right) \\
L \quad \longmapsto L_{F}
\end{gathered}
$$

que é uma bijeção linear, cuja inversa é

$$
\begin{gathered}
\mathfrak{X}\left(\Omega_{2}\right) \quad \longrightarrow \mathfrak{X}\left(\Omega_{1}\right) \\
L \quad \longmapsto L_{F^{-1}}
\end{gathered}
$$

Obs. 1.5.23. Dizemos que $L_{F}$ é o campo $L$ em $\Omega_{2}$ induzido por $F$.

Proposição 1.5.24. $\operatorname{Ker}\left(L_{F}\right)=\left(F^{-1}\right)^{*}(\operatorname{Ker}(L))$. Ou seja, "a procura de soluções para um campo não depende de sua representação em coordenadas".

Demonstração: Seja $u \in \mathscr{D}^{\prime}\left(\Omega_{2}\right)$. Então

$$
\begin{aligned}
u \in \operatorname{Ker}\left(L_{F}\right) & \Longleftrightarrow L_{F}(u)=0 \\
& \Longleftrightarrow\left(F^{-1}\right)^{*}\left(L\left(F^{*}(u)\right)\right)=0 \\
& \Longleftrightarrow L\left(F^{*}(u)\right)=0 \\
& \Longleftrightarrow F^{*}(u) \in \operatorname{Ker}(L) \\
& \Longleftrightarrow u \in\left(F^{-1}\right)^{*}(\operatorname{Ker}(L))
\end{aligned}
$$


Existe uma relação especial entre o símbolo de $L$ e o símbolo de $L_{F}$. Seja $x \in \Omega_{1}$. Dado $\xi=\left(\xi_{1}, \xi_{2}\right) \in \mathbb{R}^{2}$, temos

$$
\begin{gathered}
L_{F}(F(x), \xi)=L_{F}\left(\pi_{1}\right)(F(x)) \xi_{1}+L_{F}\left(\pi_{2}\right)(F(x)) \xi_{2}= \\
=\left(L\left(F_{1}\right) \circ F^{-1}\right)(F(x)) \xi_{1}+\left(L\left(F_{2}\right) \circ F^{-1}\right)(F(x)) \xi_{2} \\
=L\left(F_{1}\right)(x) \xi_{1}+L\left(F_{2}\right)(x) \xi_{2} \\
=\left[L\left(\pi_{1}\right)(x) \frac{\partial F_{1}}{\partial x_{1}}(x)+L\left(\pi_{2}\right)(x) \frac{\partial F_{1}}{\partial x_{2}}(x)\right] \xi_{1}+\left[L\left(\pi_{1}\right)(x) \frac{\partial F_{2}}{\partial x_{1}}(x)+L\left(\pi_{2}\right)(x) \frac{\partial F_{2}}{\partial x_{2}}(x)\right] \xi_{2} \\
=L\left(\pi_{1}\right)(x)\left[\frac{\partial F_{1}}{\partial x_{1}}(x) \xi_{1}+\frac{\partial F_{2}}{\partial x_{1}}(x) \xi_{2}\right]+L\left(\pi_{2}\right)(x)\left[\frac{\partial F_{1}}{\partial x_{2}}(x) \xi_{1}+\frac{\partial F_{2}}{\partial x_{2}}(x) \xi_{2}\right] \\
=L(x, \eta(x, \xi)),
\end{gathered}
$$

onde $\eta(x, \xi)=\left(\eta_{1}(x, \xi), \eta_{2}(x, \xi)\right)$, com

$$
\eta_{1}(x, \xi)=\left[\frac{\partial F_{1}}{\partial x_{1}}(x) \xi_{1}+\frac{\partial F_{2}}{\partial x_{1}}(x) \xi_{2}\right] \text { e } \eta_{2}(x, \xi)=\left[\frac{\partial F_{1}}{\partial x_{2}}(x) \xi_{1}+\frac{\partial F_{2}}{\partial x_{2}}(x) \xi_{2}\right] .
$$

Note que

$$
J(F)(x)=\left[\begin{array}{ll}
\frac{\partial F_{1}}{\partial x_{1}}(x) & \frac{\partial F_{1}}{\partial x_{2}}(x) \\
\frac{\partial F_{2}}{\partial x_{1}}(x) & \frac{\partial F_{2}}{\partial x_{2}}(x)
\end{array}\right]
$$

Logo, $\eta(x, \xi)=(J(F)(x))^{t}(\xi)$. Assim

$$
L_{F}(F(x), \xi)=L\left(x,(J(F)(x))^{t}(\xi)\right) \text {, para todo } x \in \Omega \text { e } \xi \in \mathbb{R}^{2} .
$$

Observe que $\eta(x, \xi)=0 \Longleftrightarrow \xi=0$, pois $\operatorname{det}(J(F)(x)) \neq 0$, uma vez que $F$ é um difeomorfismo. Daí segue que $L_{F}$ é elítico em $y$ se, e somente se, $L$ é elítico em $x$. E assim está demonstrada a propriedade abaixo.

Proposição 1.5.25. L é elítico em $\Omega_{1}$ se, e somente se, $L_{F}$ é elítico em $\Omega_{2}$.

Ou seja, o conceito de eliticidade é definido de modo que independe das "coordenadas que o campo está representado". Analogamente, a hipoelicidade tem a mesma propriedade: 
Proposição 1.5.26. L é hipoelítico em $\Omega_{1}$ se, e somente se, $L_{F}$ é hipoelítico em $\Omega_{2}$.

\section{Demonstração:}

Suponha que $L$ seja hipoelítico em $\Omega_{1}$. Seja $u \in \mathscr{D}^{\prime}\left(\Omega_{2}\right)$ tal que $L_{F}(u) \in C^{\infty}(V)$, onde $V \subset \Omega_{2}$ aberto. Ou seja, $\left(F^{-1}\right)^{*}\left(L\left(F^{*}(u)\right)\right) \in C^{\infty}(V)$. Seja $U \subset \Omega_{1}$ tal que $F(U)=V$. Assim $L\left(F^{*}(u)\right) \in C^{\infty}(U)$. Pela hipoeliticidade de $L, F^{*}(u) \in C^{\infty}(U)$. Logo $u \in C^{\infty}(V)$. Portanto $L_{F}$ é hipoelítico. Reciprocamente, se $L_{F}$ é hipoelítico em $\Omega_{2}$, pelo que já foi mostrado, $L=\left(L_{F}\right)_{F^{-1}}$ é hipoelítco em $\Omega_{1}$.

Definição 1.5.27. Dizemos que um campo $L$ definido em $\Omega$ é localmente integrável se associada a ele existe uma cobertura aberta $\mathcal{U}$ de $\Omega$, tal que para cada $U \in \mathcal{U}$, existe $Z_{U}: U \longrightarrow \mathbb{C}$ função $C^{\infty}$ que satisfaz $L Z_{U}=0$ e $d Z_{U} \neq 0$ em $U$.

Lema 1.5.28. Seja $L$ um campo vetorial complexo em $\Omega$ e $Z_{U}: U \longrightarrow \mathbb{C}$ uma função de classe $C^{\infty}$ que satisfaz $L Z_{U}=0$ e $d Z_{U} \neq 0$ em $U$, onde $U$ é um aberto de $\Omega$. Dado $p \in U$, existem:

1. polinômio $Q$ de grau 1

2. um difeomorfismo $\Phi: V \longrightarrow B$, onde $B$ é uma vizinhança da origem, $V$ é uma vizinhança de $p$ com $V \subset U$, tal que $\Phi(p)=(0,0) e$

$\Phi^{-1^{*}}\left(\left.Z_{U}\right|_{V}\right)=Z_{U} \circ \Phi^{-1}, Z_{U}$ nas novas variáveis $y_{1}, y_{2}$ em $V$, induzidas por $\Phi^{-1}$, satisfaz

$$
\left(Q \circ Z_{U} \circ \Phi^{-1}\right)\left(y_{1}, y_{2}\right)=y_{1}+i \varphi\left(y_{1}, y_{2}\right)
$$

onde $\varphi$ é uma função a valores reais que satisfaz: $\varphi(0,0)=0$ e $\frac{\partial \varphi}{\partial y_{1}}(0,0)=0$.

\section{Demonstração:}

Como $d Z_{U} \neq 0$ em $p$, então

$$
\frac{\partial Z_{U}}{\partial x}(p)=\frac{\partial \Re\left\{Z_{U}\right\}}{\partial x}(p)+i \frac{\partial \Im\left\{Z_{U}\right\}}{\partial x}(p) \neq 0
$$

$\mathrm{Ou}$

$$
\frac{\partial Z_{U}}{\partial t}(p)=\frac{\partial \Re\left\{Z_{U}\right\}}{\partial t}(p)+i \frac{\partial \Im\left\{Z_{U}\right\}}{\partial t}(p) \neq 0 .
$$


Sem perda de generalidade, suponha que $\frac{\partial \Re\left\{Z_{U}\right\}}{\partial x}(p) \neq 0$.

Seja $r>0$ tal que $B_{r}(p) \subset U$. Considere

$$
\begin{aligned}
T: B_{r}(p) & \longrightarrow \\
(x, t) & \longmapsto\left(x-p_{1}, t-p_{2}\right)
\end{aligned}
$$

Considere $\left(x_{1}, x_{2}\right)$ um elemento genérico de $B_{r}(0)$. A partir de $T$, definimos $\tilde{Z} \doteq Z_{U} \circ T^{-1}$ : $B_{r}(0) \longrightarrow \mathbb{C}$.

Seja $g: \mathbb{C} \longrightarrow \mathbb{C}$, uma translação dada por $g(z)=z-\tilde{Z}(0,0), z \in \mathbb{C}$.

Considere $Z^{*} \doteq(g \circ \tilde{Z})$ em $B_{r}(0)$. Note que $Z^{*}(0,0)=0$. Observe que

$$
\frac{\partial \Re Z^{*}}{\partial x_{1}}\left(x_{1}, x_{2}\right)=\frac{\partial \Re\{\tilde{Z}\}}{\partial x_{1}}\left(x_{1}, x_{2}\right)=\frac{\partial \Re\{Z\}}{\partial x}\left(x_{1}+p_{1}, x_{2}+p_{2}\right),
$$

para todo $\left(x_{1}, x_{2}\right) \in B_{r}(0)$. Assim $\frac{\partial \Re\left\{Z^{*}\right\}}{\partial x_{1}}(0,0)=\frac{\partial \Re\{Z\}}{\partial x}(p) \neq 0$. Logo, fica bem definida a rotação $f(z)=e^{-i \theta} z, z \in \mathbb{C}$, onde

$$
\theta \doteq \arg \left\{\frac{\partial \Re\left\{Z^{*}\right\}}{\partial x_{1}}(0,0)+i \frac{\partial \Im\left\{Z^{*}\right\}}{\partial x_{1}}(0,0)\right\}
$$

É fácil ver que

$$
f\left(\frac{\partial \Re\left\{Z^{*}\right\}}{\partial x_{1}}(0,0)+i \frac{\partial \Im\left\{Z^{*}\right\}}{\partial x_{1}}(0,0)\right)=\left|\frac{\partial \Re\left\{Z^{*}\right\}}{\partial x_{1}}(0,0)+i \frac{\partial \Im\left\{Z^{*}\right\}}{\partial x_{1}}(0,0)\right| \neq 0 .
$$

Seja $Q(z)=(f \circ g)(z), z \in \mathbb{C}$. Note que $Q$ é um polinômio de grau 1 na variável $z$.

Observe que

$$
\begin{aligned}
& \text { - } \frac{\partial \Re\left\{Q \circ \tilde{Z}_{U}\right\}}{\partial x_{1}}(0,0)=\frac{\partial \Re\left\{f \circ Z^{*}\right\}}{\partial x_{1}}(0,0)=\cos (\theta) \frac{\partial \Re\left\{Z^{*}\right\}}{\partial x_{1}}(0,0)+\operatorname{sen}(\theta) \frac{\partial \Im\left\{Z^{*}\right\}}{\partial x_{1}}(0,0) \\
& =\Re\left\{e^{-i \theta}\left(\frac{\partial \Re\left\{Z^{*}\right\}}{\partial x_{1}}(0,0)+i \frac{\partial \Im\left\{Z^{*}\right\}}{\partial x_{1}}(0,0)\right)\right\}=\Re\left\{f\left(\frac{\partial \Re\left\{Z^{*}\right\}}{\partial x_{1}}(0,0)+i \frac{\partial \Im\left\{Z^{*}\right\}}{\partial x_{1}}(0,0)\right)\right\} \\
& =\Re\left\{\left|\frac{\partial \Re\left\{Z^{*}\right\}}{\partial x_{1}}(0,0)+i \frac{\partial \Im\left\{Z^{*}\right\}}{\partial x_{1}}(0,0)\right|\right\}=\left|\frac{\partial \Re\left\{Z^{*}\right\}}{\partial x_{1}}(0,0)+i \frac{\partial \Im\left\{Z^{*}\right\}}{\partial x_{1}}(0,0)\right| \neq 0 . \\
& \text { - } \frac{\partial \Im\{Q \circ \tilde{Z}\}}{\partial x_{1}}(0,0)=\frac{\partial \Im\left\{f \circ Z^{*}\right\}}{\partial x_{1}}(0,0)=\cos (\theta) \frac{\partial \Im\left\{Z^{*}\right\}}{\partial x_{1}}(0,0)-\operatorname{sen}(\theta) \frac{\partial \Re\left\{Z^{*}\right\}}{\partial x_{1}}(0,0)=
\end{aligned}
$$




$$
\begin{aligned}
& =\Im\left\{e^{-i \theta}\left(\frac{\partial \Re\left\{Z^{*}\right\}}{\partial x_{1}}(0,0)+i \frac{\partial \Im\left\{Z^{*}\right\}}{\partial x_{1}}(0,0)\right)\right\}=\Im\left\{f\left(\frac{\partial \Re\left\{Z^{*}\right\}}{\partial x_{1}}(0,0)+i \frac{\partial \Im\left\{Z^{*}\right\}}{\partial x_{1}}(0,0)\right)\right\} \\
& =\Im\left\{\left|\frac{\partial \Re\left\{Z^{*}\right\}}{\partial x_{1}}(0,0)+i \frac{\partial \Im\left\{Z^{*}\right\}}{\partial x_{1}}(0,0)\right|\right\}=0 .
\end{aligned}
$$

Considere $F: B_{r}(0) \longrightarrow \mathbb{R}^{2}$, dada por $F\left(x_{1}, x_{2}\right)=\left(\Re\{Q \circ \tilde{Z}\}\left(x_{1}, x_{2}\right), x_{2}\right)$. Temos que $F$ é diferenciável e

$$
F^{\prime}(0,0)(y, w)=\left(\Re\{Q \circ \tilde{Z}\}^{\prime}(0,0) \cdot(y, w), w\right)=\left(\frac{\partial \Re\{Q \circ \tilde{Z}\}}{\partial x_{1}}(0,0) y+\frac{\partial \Re\{Q \circ \tilde{Z}\}}{\partial x_{2}}(0,0) w, w\right),
$$

para $(y, w) \in \mathbb{R}^{2}$. Ora, a transformação linear

$$
\xi(\alpha, \beta)=\left(\left[\frac{\partial \Re\{Q \circ \tilde{Z}\}}{\partial x_{1}}(0,0)\right]^{-1}\left(\alpha-\frac{\partial \Re\{Q \circ \tilde{Z}\}}{\partial x_{2}}(0,0) \beta\right), \beta\right)
$$

para $(\alpha, \beta) \in \mathbb{R}^{2}$, é evidentemente uma inversa de $F^{\prime}(0,0)$. Logo $F^{\prime}(0,0)$ é um isomorfismo. Pelo Teorema da Aplicação Inversa, $F$ é um difeomorfismo $C^{\infty}$ de um aberto $A$ contendo $(0,0)$ sobre um aberto $B$ contendo $(\Re\{Q \circ \tilde{Z}\}(0,0), 0)=(0,0)$.

Como $F$ deixa fixa a segunda coordenada, seu inverso $F^{-1}$ tem a mesma propriedade, i.e., $F^{-1}\left(y_{1}, y_{2}\right)=\left(F_{1}^{-1}\left(y_{1}, y_{2}\right), y_{2}\right)$ para todo $\left(y_{1}, y_{2}\right) \in B$. Então, para $\left(y_{1}, y_{2}\right) \in B$ temos

$$
\begin{aligned}
\left(x_{1}, x_{2}\right) & =F\left(F^{-1}\left(x_{1}, x_{2}\right)\right)=F\left(\left(F_{1}^{-1}\left(x_{1}, x_{2}\right), x_{2}\right)\right) \\
& =\left(\Re\{Q \circ \tilde{Z}\}\left(F_{1}^{-1}\left(y_{1}, y_{2}\right), y_{2}\right), y_{2}\right) \\
& =\left(\left(\Re\{Q \circ \tilde{Z}\} \circ F^{-1}\right)\left(y_{1}, y_{2}\right), y_{2}\right) .
\end{aligned}
$$

Ou seja, $\left(\Re\{Q \circ \tilde{Z}\} \circ F^{-1}\right)\left(y_{1}, y_{2}\right)=y_{1}$, para todo $\left(y_{1}, y_{2}\right) \in B$.

Seja $V=T^{-1}(A)$. Se necessário podemos reduzir $A$ de modo que $\bar{V} \subset U$. Como $F$ e $T$ são difeomorfismos, temos que

$$
\Phi \doteq F \circ T: V \longrightarrow B
$$


é um difeomorfismo. Além disso,

$$
\begin{aligned}
\left(Q \circ Z \circ \omega^{-1}\right)\left(y_{1}, y_{2}\right) & =\left(Q \circ \tilde{Z} \circ F^{-1}\right)\left(y_{1}, y_{2}\right) \\
& =\Re\left\{Q \circ \tilde{Z} \circ F^{-1}\right\}\left(y_{1}, y_{2}\right)+i \Im\left\{Q \circ \tilde{Z} \circ F^{-1}\right\}\left(y_{1}, y_{2}\right) \\
& =y_{1}+i \Im\left\{Q \circ \tilde{Z} \circ F^{-1}\right\}\left(y_{1}, y_{2}\right),
\end{aligned}
$$

para todo $\left(y_{1}, y_{2}\right) \in B$. Seja $\varphi\left(y_{1}, y_{2}\right) \doteq \Im\left\{Q \circ \tilde{Z} \circ F^{-1}\right\}\left(y_{1}, y_{2}\right),\left(y_{1}, y_{2}\right) \in B$. Temos

$$
\varphi(0,0)=\Im\left\{Q \circ \tilde{Z} \circ F^{-1}\right\}(0,0)=\Im\{Q \circ \tilde{Z}\}(0,0)=0
$$

e

$$
\begin{aligned}
\frac{\partial \varphi}{\partial y_{1}}\left(y_{1}, y_{2}\right) & =\frac{\partial \Im\left\{Q \circ \tilde{Z} \circ F^{-1}\right\}}{\partial y_{1}}\left(y_{1}, y_{2}\right)=\frac{\partial\left(\Im\{Q \circ \tilde{Z}\} \circ F^{-1}\right)}{\partial y_{1}}\left(y_{1}, y_{2}\right) \\
& =\frac{\partial \Im\{Q \circ \tilde{Z}\}}{\partial x_{1}}\left(y_{1}, y_{2}\right) \frac{\partial F_{1}^{-1}}{\partial y_{1}}\left(y_{1}, y_{2}\right)+\frac{\partial \Im\{Q \circ \tilde{Z}\}}{\partial x_{2}}\left(y_{1}, y_{2}\right) \frac{\partial F_{2}^{-1}}{\partial y_{1}}\left(y_{1}, y_{2}\right) \\
& =\frac{\partial \Im\{Q \circ \tilde{Z}\}}{\partial x_{1}}\left(y_{1}, y_{2}\right) \frac{\partial F_{1}^{-1}}{\partial y_{1}}\left(y_{1}, y_{2}\right)+\frac{\partial \Im\{Q \circ \tilde{Z}\}}{\partial x_{2}}\left(y_{1}, y_{2}\right) \frac{\partial y_{2}}{\partial y_{1}}\left(y_{1}, y_{2}\right) \\
& =\frac{\partial \Im\{Q \circ \tilde{Z}\}}{\partial x_{1}}\left(y_{1}, y_{2}\right) \frac{\partial F_{1}^{-1}}{\partial y_{1}}\left(y_{1}, y_{2}\right) .
\end{aligned}
$$

Assim, como $\frac{\partial \Im\{Q \circ \tilde{Z}\}}{\partial x_{1}}(0,0)=0$, temos que $\frac{\partial \varphi}{\partial y_{1}}(0,0)=0$. Portanto $Q$ e $\Phi$ são o polinômio e o difeomorfismo procurados.

Obs. 1.5.29. Em particular, $L_{\Phi}\left(Z_{U} \circ \Phi^{-1}\right)=0 \mathrm{em} B$.

Agora vamos estudar outro tipo de composição. Inicialmente, considere o homeomorfismo

$$
\begin{aligned}
\iota: \mathbb{R}^{2} & \longrightarrow \mathbb{C} \\
\left(x_{1}, x_{2}\right) & \longmapsto x_{1}+i x_{2}
\end{aligned}
$$


Seja $\Omega \subset \mathbb{R}^{2}$ um aberto e $L$ um campo em $\Omega$. Sejam $f \in C^{\infty}(\Omega)$ e $h: U \longrightarrow \mathbb{C}$, onde $U \subset \mathbb{C}$ é um aberto que contém a imagem de $f$, i.e., $f(\Omega) \subset U$. Suponha que $h \circ \iota \in C^{\infty}\left(\iota^{-1}(\Omega)\right)$, i.e.,

$$
\tilde{h}\left(y_{1}, y_{2}\right) \doteq(h \circ \iota)\left(y_{1}, y_{2}\right)=h\left(y_{1}+i y_{2}\right)
$$

$\left(y_{1}, y_{2}\right) \in \iota^{-1}(\Omega)=\left\{\left(y_{1}, y_{2}\right) \in \mathbb{R}^{2} ; y_{1}+i y_{2} \in \Omega\right\}$ é $C^{\infty}$.

Agora, seja $F \doteq\left(\iota^{-1} \circ f\right)=(\Re\{f\}, \Im\{f\})$, em $\Omega$.

Então $h \circ f=h \circ\left(\iota \circ \iota^{-1}\right) \circ f=(h \circ \iota) \circ\left(\iota^{-1} \circ f\right)=\tilde{h} \circ F$ em $\Omega$. Assim,

$$
\frac{\partial(h \circ f)}{\partial x_{j}}=\frac{\partial(\tilde{h} \circ F)}{\partial x_{j}}=\frac{\partial \tilde{h}}{\partial y_{1}}(F) \frac{\partial \Re\{f\}}{\partial x_{j}}+\frac{\partial \tilde{h}}{\partial y_{2}}(F) \frac{\partial \Im\{f\}}{\partial x_{j}}
$$

para $j=1,2$.

Assim,

$L(h \circ f)=$

$=L\left(\pi_{1}\right) \frac{\partial(h \circ f)}{\partial x_{1}}+L\left(\pi_{2}\right) \frac{\partial(h \circ f)}{\partial x_{2}}$

$=L\left(\pi_{1}\right)\left(\frac{\partial \tilde{h}}{\partial y_{1}}(F) \frac{\partial \Re\{f\}}{\partial x_{1}}+\frac{\partial \tilde{h}}{\partial y_{2}}(F) \frac{\partial \Im\{f\}}{\partial x_{1}}\right)+L\left(\pi_{2}\right)\left(\frac{\partial \tilde{h}}{\partial y_{1}}(F) \frac{\partial \Re\{f\}}{\partial x_{2}}+\frac{\partial \tilde{h}}{\partial y_{2}}(F) \frac{\partial \Im\{f\}}{\partial x_{2}}\right)$

$=\frac{\partial \tilde{h}}{\partial y_{1}}(F)\left(L\left(\pi_{1}\right) \frac{\partial \Re\{f\}}{\partial x_{1}}+L\left(\pi_{2}\right) \frac{\partial \Re\{f\}}{\partial x_{2}}\right)+\frac{\partial \tilde{h}}{\partial y_{2}}(F)\left(L\left(\pi_{1}\right) \frac{\partial \Im\{f\}}{\partial x_{1}}+L\left(\pi_{2}\right) \frac{\partial \Im\{f\}}{\partial x_{2}}\right)$

$=\frac{\partial \tilde{h}}{\partial y_{1}}(F) L(\Re\{f\})+\frac{\partial \tilde{h}}{\partial y_{2}}(F) L(\Im\{f\})$.

Conside

$$
\frac{\partial}{\partial \bar{w}}=\frac{1}{2}\left(\frac{\partial}{\partial y_{1}}+i \frac{\partial}{\partial y_{2}}\right) \quad \text { e } \quad \frac{\partial}{\partial w}=\frac{1}{2}\left(\frac{\partial}{\partial y_{1}}-i \frac{\partial}{\partial y_{2}}\right)
$$

onde $w=y_{1}+i y_{2},\left(y_{1}, y_{2}\right) \in \iota^{-1}(U)$. Assim, podemos escrever

$$
\frac{\partial}{\partial y_{1}}=\frac{\partial}{\partial w}+\frac{\partial}{\partial \bar{w}} \quad \text { e } \quad \frac{\partial}{\partial y_{2}}=i\left(\frac{\partial}{\partial w}-\frac{\partial}{\partial \bar{w}}\right)
$$


Daí,

$$
\begin{aligned}
L(h \circ f) & =\left(\frac{\partial \tilde{h}}{\partial w}+\frac{\partial \tilde{h}}{\partial \bar{w}}\right)(F) L(\Re\{f\})+i\left(\frac{\partial \tilde{h}}{\partial w}-\frac{\partial \tilde{h}}{\partial \bar{w}}\right)(F) L(\Im\{f\}) \\
& =\frac{\partial \tilde{h}}{\partial w}(F)(L(\Re\{f\})+i L(\Im\{f\}))+\frac{\partial \tilde{h}}{\partial \bar{w}}(F)(L(\Re\{f\})-i L(\Im\{f\})) \\
& =\frac{\partial \tilde{h}}{\partial w}(F)(L(\Re\{f\}+i \Im\{f\}))+\frac{\partial \tilde{h}}{\partial \bar{w}}(F)(L(\Re\{f\}-i \Im\{f\})) \\
& =\frac{\partial \tilde{h}}{\partial w}(F) L(f)+\frac{\partial \tilde{h}}{\partial \bar{w}}(F) L(\bar{f}) .
\end{aligned}
$$

Assim temos

$$
L(h \circ f)=\frac{\partial(h \circ \iota)}{\partial w}\left(\iota^{-1} \circ f\right) L(f)+\frac{\partial(h \circ \iota)}{\partial \bar{w}}\left(\iota^{-1} \circ f\right) L(\bar{f})
$$

Segue da igualdade acima a seguinte proposição:

Proposição 1.5.30. Se $f \in C^{\infty}(\Omega)$, e $h: U \longrightarrow \mathbb{C}$ é holomorfa, com $f(\Omega) \subset U$, então

$$
L(h \circ f)=\left(h^{\prime} \circ f\right) L(f)
$$

em $\Omega$. Em particular, quando $f$ é solução de L, i.e., $L(f)=0$, então $h \circ f$ é solução, i.e., $L(h \circ f)=0$.

\section{Demonstração:}

Se $h$ é holomorfa, então $\frac{\partial \tilde{h}}{\partial \bar{w}}=0$. De (1.5.1) temos

$$
\begin{aligned}
L(h \circ f) & =\frac{\partial \tilde{h}}{\partial w}(F) L(f)=\frac{\partial \tilde{h}}{\partial w}\left(\iota^{-1} \circ f\right) L(f)= \\
& =\left[\left(\frac{\partial \tilde{h}}{\partial w} \circ \iota^{-1}\right) \circ f\right] L(f)=\left(h^{\prime} \circ f\right) L(f) .
\end{aligned}
$$




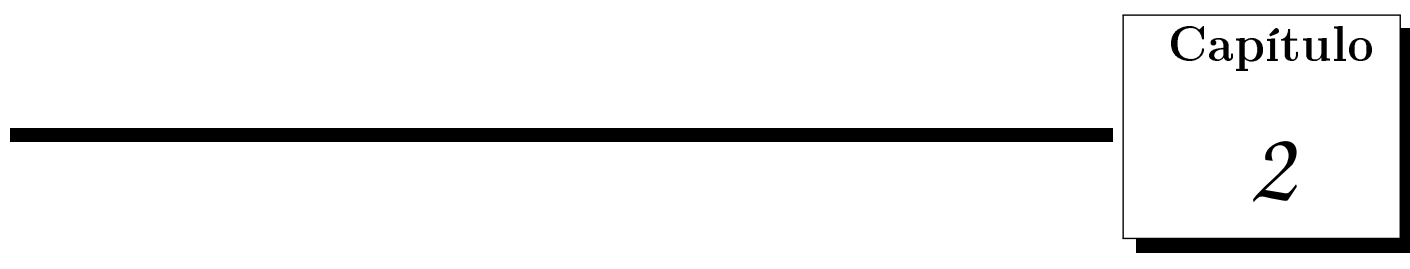

\section{Superfícies de Riemann}

Este capítulo se encaixa como um pré-requisito neste trabalho. No entanto, este assunto ser tratado como um capítulo independente do capítulo de pré-requisitos, faz jus ao fato de ser incomum a utilização, das ferramentas descritas aqui, no estudo de campos vetoriais.

\subsection{Definição}

Definição 2.1.1. Seja $M$ um espaço topológico. Uma estrutura diferenciável complexa em $M$ é uma família de pares $\left\{\left(z_{\alpha}, U_{\alpha}\right)\right\}_{\alpha \in \mathcal{I}}$, chamados de cartas, onde $U_{\alpha}$ é um aberto de $M$ e $z_{\alpha}: U_{\alpha} \longrightarrow z_{\alpha}\left(U_{\alpha}\right)$ é um homeomorfismo sobre um aberto de $\mathbb{C}$ para todo $\alpha \in I$ e que satisfaz as seguintes propriedades:

A. 1. $M=\bigcup_{\alpha \in \mathcal{I}} U_{\alpha}$

A. 2. se $U_{\alpha} \cap U_{\beta} \neq 0$, então

$$
z_{\alpha} \circ z_{\beta}^{-1}: z_{\beta}\left(U_{\alpha} \cap U_{\beta}\right) \longrightarrow z_{\alpha}\left(U_{\alpha} \cap U_{\beta}\right)
$$

é uma função holomorfa. 
A. 3. A familia $\left\{\left(z_{\alpha}, U_{\alpha}\right)\right\}_{\alpha \in \mathcal{I}}$ é maximal com respeito a $A$. 1 e A. 2, isto é, se $V \subset M$ é um aberto não vazio, e $w: V \longrightarrow w(V)$ é um homeomorfismo sobre um aberto de $\mathbb{C}$ tal que para cada $\alpha \in I$ com $U_{\alpha} \cap V \neq \emptyset$, a composição $w \circ z_{\alpha}^{-1}: z_{\alpha}\left(U_{\alpha} \cap V\right) \longrightarrow w\left(U_{\alpha} \cap V\right)$ é holomorfa, então $(w, V) \in\left\{\left(z_{\alpha}, U_{\alpha}\right)\right\}_{\alpha \in I}$.

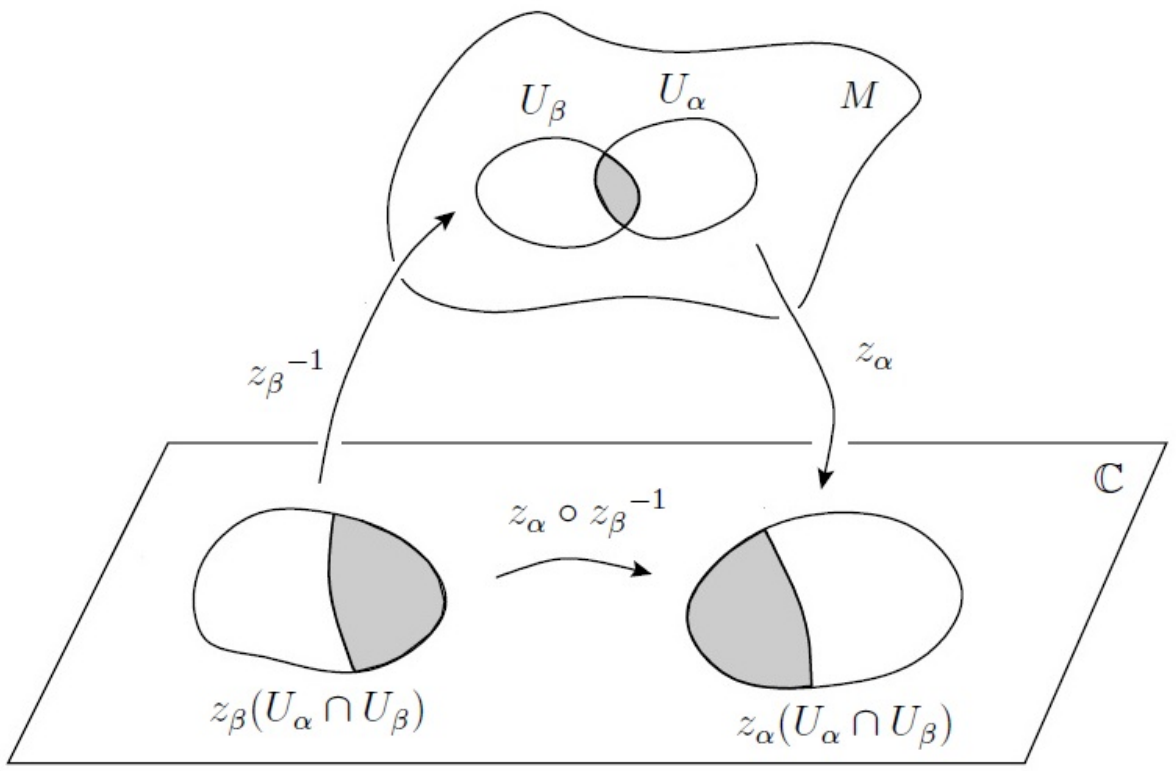

Um atlas (complexo) em $M$ é uma família que satisfaz as condições A. 1 e A. 2 acima. Em particular, uma estrutura diferenciável complexa em $M$ é um atlas em $M$, às vezes chamado de atlas (complexo) maximal.

Definição 2.1.2. Uma Superfície de Riemann é um espaço topológico de Hausdorff conexo M, com base enumerável e munido de uma estrutura diferenciável complexa.

A verificação da condição A. 3 pode ser difícil em certos casos. Assim, uma importante observação é que a condição A. 3 não é essencial na definição de estrutura diferenciável complexa, e consequentemente na definição de Superfície de Riemann. De fato, se apenas as condições A. 1 e A. 2 são satisfeitas, isto é, se $\mathcal{A}$ é um atlas em $M$, então $\mathcal{A}$ pode ser estendido a uma única família maximal $\mathscr{A}$ de modo que $\mathscr{A}$ contém $\mathcal{A}$ e satisfaz A. 1, A. 2 e A. 3. Esta família maximal, $\mathscr{A}$, é formada por todos os pares $(V, \zeta)$, onde $V \subset M$ é um aberto não vazio, e $\zeta: V \longrightarrow \zeta(V)$ é um homeomorfismo sobre um aberto de $\mathbb{C}$ tal 
que para cada $\alpha \in I \operatorname{com} U_{\alpha} \cap V \neq \emptyset$, a composição $\zeta \circ z_{\alpha}^{-1}: z_{\alpha}\left(U_{\alpha} \cap V\right) \longrightarrow \zeta\left(U_{\alpha} \cap V\right)$ é holomorfa. Neste caso, dizemos que o atlas $\mathcal{A}$ gera a estrutura diferenciável $\mathscr{A}$ em $M$.

Obs. 2.1.3. Se $M$ é um espaço topológico Hausdorff conexo munido de uma estrutura diferenciável complexa, então automaticamente $M$ possui base enumerável. A difícil prova dessa afirmação pode ser encontrada em [10].

Exemplo 2.1.4. O plano complexo $\mathbb{C}$, com a estrutura diferenciável complexa gerada pelo atlas unitário $\{(\mathbb{C}, i d)\}$, onde $i d: \mathbb{C} \longrightarrow \mathbb{C}$, é a aplicação identidade de $\mathbb{C}$, é um exemplo simples de superfície de Riemann. Outros dois exemplos de mesma natureza, são o disco unitário com centro na origem, $\mathbb{D}=\{z \in \mathbb{C} ;|z|<1\}$, e o semiplano superior, $\mathbb{H}=\{z \in \mathbb{C} ; \Im\{z\}>0\}$, ambos com a estrutura diferenciável gerada pelo atlas unitário constituído da aplicação identidade restrita a cada um.

Intuitivamente, uma superfície de Riemann pode ser pensada como um espaço que é localmente o plano complexo.

Exemplo 2.1.5. Seja $M$ uma superfície de Riemann com estrutura diferenciável complexa $\mathscr{A}$ e $U$ um aberto de $M$. Considere $U$ a topologia induzida por $M$. Para cada $\left(U_{\alpha}, z_{\alpha}\right) \in$ $\mathscr{A}$, considere $\tilde{U}_{\alpha}=U \cap U_{\alpha}$ e $\tilde{z}_{\alpha}=\left.z_{\alpha}\right|_{\tilde{U}_{\alpha}}$. Considere o conjunto

$$
\mathcal{A}_{U}=\left\{\left(\tilde{U}_{\alpha}, \tilde{z}_{\alpha}\right) ;\left(U_{\alpha}, z_{\alpha}\right) \in \mathscr{A}\right\}
$$

É fácil ver que $\mathcal{A}_{U}$ é um atlas em $U$. Assim, $U$ com a estrutura diferenciável complexa induzida por $\mathcal{A}_{U}$ é uma superfície de Riemann.

Exemplo 2.1.6. Em vários problemas envolvendo a teoria das variáveis complexas é conveniente tratar o "infinito" como um ponto ordinário, ou seja, estendendo o conjunto $\mathbb{C}$ dos números complexos com a introdução do símbolo $\infty$ que representará o que chamamos de "infinito". O conjunto

$$
\overline{\mathbb{C}}=\mathbb{C} \cup\{\infty\}
$$

é chamado de Esfera de Riemann devido ao modelo geométrico construído por Riemann o qual relaciona $\overline{\mathbb{C}}$ com $\mathbb{S}^{2}=\left\{\left(x_{1}, x_{2}, x_{3}\right) ; x_{1}^{2}+x_{2}^{2}+x_{3}^{2}=1\right\}$ a esfera unitária do $\mathbb{R}^{3}$. Discutiremos este modelo nos exemplos 2.1.7 e 2.1.10. Considere $\overline{\mathbb{C}}$ com a topologia $\mathscr{T}$, 
que é constituída dos aberto de $\mathbb{C}$ e mais os conjuntos da forma $A \cup\{\infty\}$ onde $A$ é um aberto em $\mathbb{C}$ tal que $\mathbb{C} \backslash A$ é um compacto de $\mathbb{C}$. O conjunto $\overline{\mathbb{C}}$ com a topologia $\mathscr{T} e ́$ um espaço de Hausdorff e Compacto. O conjunto $\overline{\mathbb{C}}$ com a topologia $\mathscr{T}$ é chamado de compactificação de $\mathbb{C}$ por um ponto. Considere $\mathcal{A}=\left\{\left(U_{1}, z_{1}\right),\left(U_{2}, z_{2}\right)\right\}$, onde

$$
\begin{aligned}
& U_{1}=\mathbb{C} \\
& U_{2}=(\mathbb{C} \backslash\{0\}) \cup\{\infty\}
\end{aligned}
$$

$e$

$$
\begin{aligned}
& z_{1}(z)=z, \quad z \in U_{1} \\
& z_{2}(z)=\frac{1}{z}, \quad \text { se } \quad z \in \mathbb{C} \backslash\{0\} \quad \text { e } \quad z_{2}(\infty)=0 .
\end{aligned}
$$

$O$ conjunto $\mathcal{A}$ é um atlas em $\overline{\mathbb{C}}$.

Considere a aplicação $\Phi: \mathbb{S}^{2} \longrightarrow \overline{\mathbb{C}}$ dada por $\Phi\left(x_{1}, x_{2}, x_{3}\right)=\frac{x_{1}+i x_{2}}{1-x_{3}}$, se $\left(x_{1}, x_{2}, x_{3}\right) \in$ $\mathbb{S}^{2} \backslash\{(0,0,1)\}$ e $\Phi(0,0,1)=\infty$. $\Phi$ é um homeomorfismo entre os espaços $\mathbb{S}^{2} e \overline{\mathbb{C}}$, onde a topologia de $\mathbb{S}^{2}$ é a topologia induzida do $\mathbb{R}^{3}$. Daí, temos que $\overline{\mathbb{C}}$ é simplesmente conexo e possui base enumerável. Portanto, $\overline{\mathbb{C}}$ com a estrutura diferenciável complexa induzida pelo atlas $\mathcal{A}$ é uma superfície de Riemann.

Exemplo 2.1.7. Novamente, considere $\mathbb{S}^{2}$ com a topologia induzida do $\mathbb{R}^{3}$. Assim, em particular $V_{1}=\mathbb{S}^{2} \backslash\{(0,0,1)\}$ e $V_{2}=\mathbb{S}^{2} \backslash\{(0,0,-1)\}$ são abertos de $\mathbb{S}^{2}$. As funções

$$
\begin{aligned}
& \zeta_{1}: \quad V_{1} \quad \longrightarrow \mathbb{C} \\
& \left(x_{1}, x_{2}, x_{3}\right) \longmapsto \frac{x_{1}+i x_{2}}{1-x_{3}}
\end{aligned}
$$

$e$

$$
\begin{aligned}
& \zeta_{2}: \quad V_{2} \quad \longrightarrow \mathbb{C} \\
& \left(x_{1}, x_{2}, x_{3}\right) \longmapsto \frac{x_{1}-i x_{2}}{1+x_{3}}
\end{aligned}
$$

são homeomorfismos e além disso,

$$
\zeta_{1} \circ \zeta_{2}^{-1}: \zeta_{2}\left(V_{1} \cap V_{2}\right) \longrightarrow \zeta_{1}\left(V_{1} \cap V_{2}\right)
$$


é o biholomorfismo $z \mapsto 1 / z$ de $\mathbb{C} \backslash\{0\}$. Daí, $\mathcal{B}=\left\{\left(\zeta_{1}, V_{1}\right),\left(\zeta_{2}, V_{2}\right)\right\}$ é um atlas em $\mathbb{S}^{2}$, que é um espaço de Hausdorff simplesmente conexo com base enumerável e compacto. $E$ assim $\mathbb{S}^{2}$ com a estrutura diferenciável complexa induzida por $\mathcal{B}$ é uma superfície de Riemann.

Definição 2.1.8. Sejam $M$ e $N$ Superfícies de Riemann. Diz-se que uma aplicação

$$
f: M \longrightarrow N
$$

é holomorfa se para cada ponto $p \in M$ existem cartas $(U, z)$ em $M,(V, \zeta)$ em $N$, com $p \in U$ e $f(U) \subset V$ tais que

$$
\zeta \circ f \circ z^{-1}: z(U) \longrightarrow \zeta(V)
$$

é uma função holomorfa (no sentido usual da análise complexa). Uma aplicação $f$ é chamada de biholomorfismo entre superfícies de Riemann se é uma bijeção que é uma função holomorfa e com

$$
f^{-1}: N \longrightarrow M
$$

também uma função holomorfa. Neste caso, dizemos que as superfícies de Riemann são biholomorfas.

Toda função holomorfa entre superfícies de Riemann é contínua. Logo, todo biholomorfismo é um homeomorfismo. Em particular, superfícies de Riemann biholomorfas possuem o mesmo grupo fundamental.

Obs. 2.1.9. Na verdade, se $f$ é uma bijeção que é holomorfa, então $f$ é um biholomorfismo.

Exemplo 2.1.10. A aplicação definida no exemplo 2.1.6 é mais que um homeomorfismo de $\mathbb{S}^{2}$ sobre $\overline{\mathbb{C}}$, é um biholomorfismo quando $\mathbb{S}^{2}$ tem a estrutura diferenciável complexa definida no exemplo 2.1.7. De fato, se $p \in \mathbb{S}^{2} \backslash\{(0,0,1)\}$, temos que $p \in V_{1}, f\left(V_{1}\right)=U_{1}$ e fazendo as contas concluímos que

$$
z_{1} \circ \Phi \circ \zeta_{1}^{-1}: \zeta_{1}\left(V_{1}\right) \longrightarrow z_{1}\left(U_{1}\right)
$$




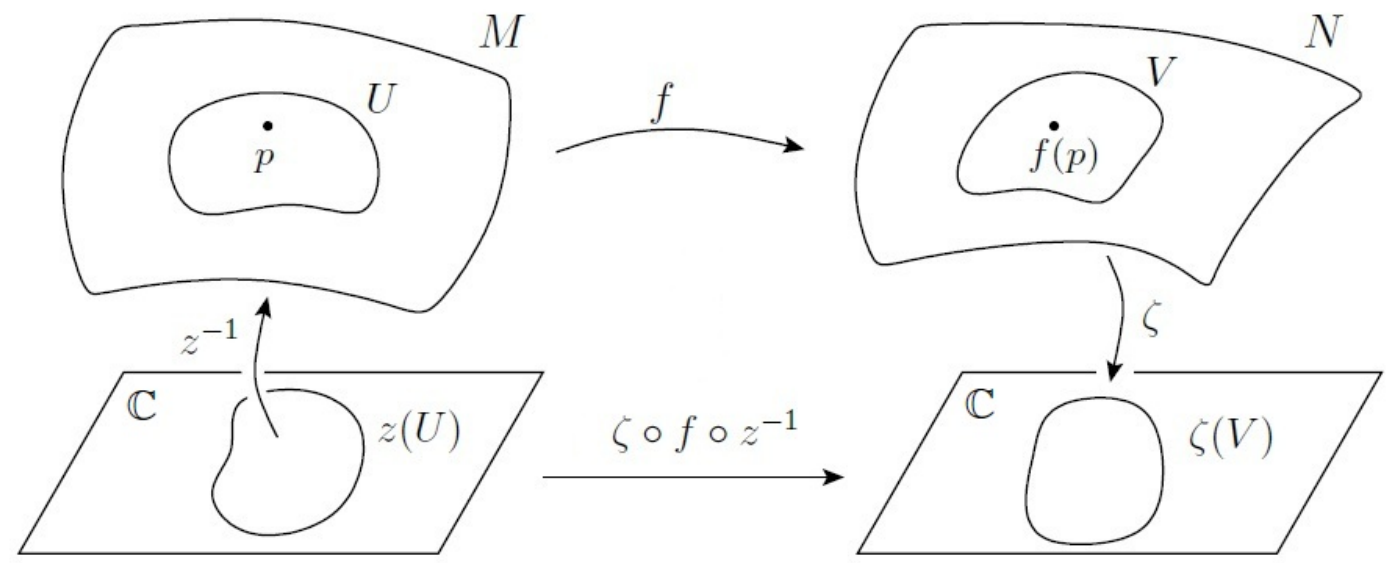

é a aplicação identidade $z \mapsto z$ de $\mathbb{C}$. Agora, se $p=(0,0,1)$, temos que $p \in V_{2}, f\left(V_{2}\right)=U_{2}$ e fazendo as contas concluímos que

$$
z_{2} \circ \Phi \circ \zeta_{2}^{-1}: \zeta_{2}\left(V_{2}\right) \longrightarrow z_{2}\left(U_{2}\right)
$$

é a aplicação identidade $z \mapsto z$ de $\mathbb{C}$. Portanto $\Phi: \mathbb{S}^{2} \longrightarrow \overline{\mathbb{C}}$ é uma bijeção que é holomorfa, ou seja, $\Phi$ é um biholomorfismo.

Exemplo 2.1.11. Qualquer biholomorfismo de $\mathbb{D}$ é uma transformação de Möbius da forma $T(z)=e^{i \gamma} \frac{z-\theta}{\bar{\theta} z-1} \operatorname{com}|\theta|<1$ e $\gamma \in \mathbb{R}$.

Exemplo 2.1.12. Qualquer biholomorfismo de $\mathbb{H}$ sobre $\mathbb{D}$ é uma transformação de Möbius da forma $T(z)=e^{i \theta_{1}} \frac{z-z_{1}}{z-\bar{z}_{1}}$, com $z_{1} \in \mathbb{H}$ e $\theta_{1} \in \mathbb{R}$.

Exemplo 2.1.13. Qualquer biholomorfismo de $\mathbb{C}$ é uma transformação afim, isto é, $T(z)=a z+b$ onde $a \neq 0$.

Exemplo 2.1.14. Não existe biholomorfismo entre quaisquer duas das superfícies de Riemann $\mathbb{C}, \mathbb{D}$ e $\overline{\mathbb{C}}$. Inicialmente, note que como $\overline{\mathbb{C}}$ é compacta, não pode existir um homeomorfismo de $\overline{\mathbb{C}}$ em $\mathbb{C}$ ou de $\overline{\mathbb{C}}$ em $\mathbb{D}$. Segue do teorema de Liouville que uma função inteira (holomorfa em todo $\mathbb{C}$ ) não pode ser limitada. Em particular, não existe função holomorfa de $\mathbb{C}$ em $\mathbb{D}$. Ou seja, $\mathbb{C}$ e $\mathbb{D}$ não são biholomorfos. 


\subsection{Uniformização}

Nesta seção vamos apresentar dois teoremas, chamados de teoremas de Uniformização.

\subsubsection{O Teorema de Uniformização para Superfícies de Riemann Simplesmente Conexas}

O próximo teorema é chamado de teorema da aplicação de Riemann.

Teorema 2.2.1. Para todo aberto simplesmente conexo $\Omega \neq \mathbb{C}$ e todo $z_{0} \in \Omega$, existe uma única transformação holomorfa injetiva $\phi$ de $\Omega$ sobre $\mathbb{D}$ tal que $\phi\left(z_{0}\right)=0$ e $\phi^{\prime}\left(z_{0}\right)$ é um número real positivo.

A demonstração deste teorema pode ser encontrada em [6].

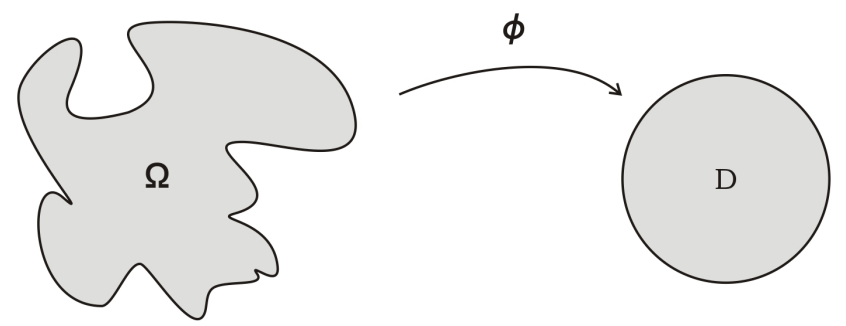

Em outras palavras, dado $\Omega \subset \mathbb{C}$ um aberto simplesmente conexo diferente de $\mathbb{C}$, então $\Omega$ e $\mathbb{D}$ são biholomorfos.

Sejam $\Omega_{1}$ e $\Omega_{2}$ abertos simplesmente conexos de $\mathbb{C}$ (vistos como superfícies de Riemann). Segue do teorema da aplicação de Riemann que $\Omega_{1}$ e $\Omega_{2}$ são biholomorfos. No entanto, quando uma das superfícies não é um aberto de $\mathbb{C}$ a situação fica um pouco mais complicada, no sentido que o teorema da aplicação de Riemann não é mais suficiente, pelo menos de forma direta, para estabelecer um biholomorfismo entre as superfícies.

O próximo teorema é chamado de teorema de uniformização de Riemann. Ele é uma extensão natural do teorema da Aplicação de Riemann para superfícies de Riemann. 
Teorema 2.2.2. Toda superfície de Riemann simplesmente conexa é biholomorfa a um dos seguintes modelos:

- a esfera de Riemann $\overline{\mathbb{C}}$;

- o plano complexo $\mathbb{C}$;

- o disco unitário $\mathbb{D}$.

A demonstração deste teorema pode ser encontrada em [8].

\subsubsection{Uniformização para Superfícies de Riemann não Simples- mente Conexas}

O que pode ser dito sobre existência de biholomorfismos entre superfícies de Riemann não simplesmente conexas? Nesta seção vamos falar um pouco do caso em que o grupo fundamental da superfície é $\mathbb{Z}$.

Um exemplo de família de superfície de Riemann com grupo fundamental igual a $\mathbb{Z}$ é a família dos anéis planos. Mais especificamente,

$$
A\left(r_{1}, r_{2}\right)=\left\{z \in \mathbb{C} ; r_{1}<|z|<r_{2}\right\}, \text { com } 0 \leq r_{1}<r_{2} \leq+\infty,
$$

com a estrutura diferenciável complexa induzida pelo atlas unitário $\left\{\left(A\left(r_{1}, r_{2}\right), i d\right)\right\}$, onde $i d: A\left(r_{1}, r_{2}\right) \longrightarrow A\left(r_{1}, r_{2}\right)$ é a aplicação identidade. Nesta família, três anéis especiais:

$$
\mathbb{C}^{*}=A(0,+\infty), \mathbb{D}^{*}=A(0,1) \text { e } A_{r}=A(r, 1), 0<r<1 .
$$

O primeiro e o segundo modelos são conhecidos como plano furado e disco unitário furado respectivamente. Os três modelos acima são especiais pois, não existe biholomorfismo entre qualquer um deles e mais, qualquer outro anel é biholomorfo a um deles. A demonstração deste resultado pode ser encontrada em [22].

Agora, se $M$ é uma superfície de Riemann com $\pi_{1}(M)=\mathbb{Z}$, existe um teorema análogo ao teorema 2.2.2? A resposta é sim, e reforça o comentário acima que os modelos $\mathbb{C}^{*}, \mathbb{D}^{*}$ e $A_{r}$ são especiais. Mais especificamente: 
Teorema 2.2.3. Seja $M$ uma superficie de Riemann com grupo fundamental $\pi_{1}(M)=\mathbb{Z}$. Então, $M$ é biholomorfa a um dos seguintes modelos:

- o plano furado $\mathbb{C}^{*}$;

- o disco unitário furado $\mathbb{D}^{*}$;

- $A_{r}=\{z \in \mathbb{C} ; r<|z|<1\}, 0<r<1$.

A demonstração deste teorema pode ser encontrada em [8].

Obs. 2.2.4. Um estudo detalhado sobre superfícies de Riemann juntamente com as demonstrações dos teoremas 2.2.2 e 2.2.3 pode ser encontrado em [8].

\subsection{Alguns comentários}

Análogo ao conceito de estrutura diferenciável complexa, existe o que chamamos de estrutura diferenciável real.

Definição 2.3.1. Seja $M$ um espaço topológico e familia de pares $\left\{\left(F_{\alpha}, U_{\alpha}\right)\right\}_{\alpha \in I}$, chamados de cartas, onde $U_{\alpha}$ é um aberto de $M$ e $F_{\alpha}: U_{\alpha} \longrightarrow F_{\alpha}\left(U_{\alpha}\right)$ é um homeomorfismo sobre um aberto de $\mathbb{R}^{2}$ para todo $\alpha \in I$ e que satisfaz as seguintes propriedades:

1. $M=\bigcup_{\alpha \in I} U_{\alpha}$

2. se $U_{\alpha} \cap U_{\beta} \neq 0$, ent $\tilde{a} O$

$$
F_{\alpha} \circ F_{\beta}^{-1}: F_{\beta}\left(U_{\alpha} \cap U_{\beta}\right) \longrightarrow F_{\alpha}\left(U_{\alpha} \cap U_{\beta}\right)
$$

é uma função holomorfa.

3. A familia $\left\{\left(F_{\alpha}, U_{\alpha}\right)\right\}_{\alpha \in I}$ é maximal com respeito a 1 e 2, isto é, se $V \subset M$ é um aberto não vazio, e $G: V \longrightarrow G(V)$ é um homeomorfismo sobre um aberto de $\mathbb{R}^{2}$ tal que para cada $\alpha \in I$ com $U_{\alpha} \cap V \neq \emptyset$, a composição $G \circ F_{\alpha}^{-1}: F_{\alpha}\left(U_{\alpha} \cap V\right) \longrightarrow$ $G\left(U_{\alpha} \cap V\right)$ é diferenciável, então $(G, V) \in\left\{\left(F_{\alpha}, U_{\alpha}\right)\right\}_{\alpha \in I}$. 
A familia $\left\{\left(F_{\alpha}, U_{\alpha}\right)\right\}_{\alpha \in I}$ é dita uma estrutura diferenciável real.

Uma família que satisfaz 1 e 2 acima, é dita um atlas (real) em $M$. Em particular, uma estrutura diferenciável real em $M$ é um atlas (real) em $M$, as vezes chamado de atlas (real) maximal. Analogamente ao caso complexo, se $\mathcal{A} \mathbb{R}$ é um atlas real em $M$, então é sempre possível estender $\mathcal{A} \mathbb{R}$ uma (e única) estrutura diferenciável real $\mathscr{A} \mathbb{R}$ em $M$.

Definição 2.3.2. Se $M$ é um espaço topológico de Hausdorff com base enumerável munido de uma estrutura diferenciável real, então $M$ é o que chamamos de uma variedade diferenciável real de dimensão 2.

Associada uma superfície de Riemann sempre existe uma Variedade Diferenciável Real de dimensão 2. De fato, seja $M$ uma superfície de Riemann. Suponha que $\mathcal{A}=$ $\left\{\left(z_{\alpha}, U_{\alpha}\right)\right\}_{\alpha \in I}$ é um atlas complexo que gera a estrutura diferenciável complexa de $M$. Para cada $\alpha \in I$, considere $F_{\alpha}: U_{\alpha} \longrightarrow F_{\alpha}\left(U_{\alpha}\right) \subset \mathbb{R}^{2}$, dada por $F_{\alpha}=\left(\Re\left\{z_{\alpha}\right\}, \Im\left\{z_{\alpha}\right\}\right)$ em $U_{\alpha}$. Assim, de $\mathcal{A}$ obtemos uma nova família de pares: $\left\{\left(F_{\alpha}, U_{\alpha}\right)\right\}_{\alpha \in I}$. Com uma simples verificação, conclui-se que $\mathcal{A} \mathbb{R} \doteq\left\{\left(F_{\alpha}, U_{\alpha}\right)\right\}_{\alpha \in I}$ é um atlas real em $M$. Assim, $M$ munida da estrutura diferenciável real $\mathscr{A} \mathbb{R}$ induzida pelo atlas real $\mathcal{A} \mathbb{R}$, é uma Variedade Diferenciável Real de dimensão 2.

Obs. 2.3.3. Seja $M$ uma superficie de Riemann com a estrutura diferenciável complexa $\mathscr{A}$. Seja $(U, Z) \in \mathscr{A}$. Defina $\tilde{Z}(z)=\overline{Z(z)}$, para $z \in U$. Observe que $\tilde{Z}: U \longrightarrow \tilde{Z}(U)$ é um homeomorfismo, pois é uma composição de homeomorfismos. Note que $(U, \tilde{Z}) \notin \mathscr{A}$. De fato, temos

$$
\begin{aligned}
\tilde{Z} \circ Z^{-1}: Z(U) & \longrightarrow \tilde{Z}(U) \\
z & \longmapsto \bar{z}
\end{aligned}
$$

Como a aplicação não é holomorfa em nenhum ponto de $\mathbb{C}$, temos que $\tilde{Z} \circ Z^{-1}: Z(U) \longrightarrow$ $\tilde{Z}(U)$ não é holomorfa.

No entanto, $F: U \longrightarrow F(U) \subset \mathbb{R}^{2}$, dada por $F=(\Re\{Z\}, \Im\{Z\})$ em $U$, é um elemento de $\mathscr{A} \mathbb{R}$, i.e., $(U, F) \in \mathscr{A} \mathbb{R}$. 


\section{Capítulo}

\section{Resolubilidade}

Neste capítulo vamos discutir e apresentar alguns resultados sobre resolubilidade de operadores diferenciais parciais.

\subsection{Resolubilidade Local}

Definição 3.1.1. $P(x, D)$ é dito localmente resolúvel em $\Omega$ quando $\forall x_{0} \in \Omega$ existe um aberto $U \subset \Omega$, com $x_{0} \in U$, de modo que $\forall f \in C_{c}^{\infty}(U)$ existe $u \in \mathscr{D}^{\prime}(\Omega) \operatorname{com} P(x, D) u=f$ em $U$.

Todos os operadores diferenciais parciais com coeficientes constantes são localmente resolúveis. Isto é uma consequência direta do teorema de Malgrange-Erenpreis [30] sobre existência de solução fundamental. O estudo da resolubilidade local fica bem mais complicado quando os coeficientes do operador não são constantes.

Exemplo 3.1.2. Em 1957 Hans Lewy [16] apresentou um exemplo de operador em $\mathbb{R}^{3}$ que é não localmente resolúvel:

$$
P=\frac{1}{2}\left(\frac{\partial}{\partial x_{1}}+i \frac{\partial}{\partial x_{2}}\right)+i\left(x_{1}+i x_{2}\right) \frac{\partial}{\partial x_{3}} .
$$

Ele mostrou que existe uma função $f \in C^{\infty}\left(\mathbb{R}^{3}\right)$ tal que a equação $P u=f$ não possui solução distribucional em nenhum aberto do $\mathbb{R}^{3}$. 


\subsection{A Condição $(\mathrm{P})$}

Nesta seção falaremos um pouco sobre a condição (P) de Nirenberg - Treves e sua relação com resolubilidade. Para falar da condição $(\mathrm{P})$, primeiro, temos que estabelecer o conceito de curvas bicaracterísticas.

Definição 3.2.1. Seja $\Omega \subset \mathbb{R}^{n}$ um aberto e seja $A$ uma aplicação de classe $C^{1}$ definida num aberto do $\Omega \times \mathbb{R}^{n}$, nas variáveis $(x, \xi)$, a valores reais. Então o sistema

$$
\begin{aligned}
& \dot{x}=\operatorname{grad}_{\xi} A(x, \xi) \\
& \dot{\xi}=-\operatorname{grad}_{x} A(x, \xi),
\end{aligned}
$$

é chamado de equações de Hamilton-Jacobi. Uma curva bicaracteristica de A passando pelo ponto $\left(x_{0}, \xi_{0}\right) \in \Omega \times \mathbb{R}^{n}$ é a curva obtida da solução das equações de Hamilton-Jacobi para $A$, com condições iniciais $\left(x_{0}, \xi_{0}\right)$.

Obs. 3.2.2. A é constante ao longo de uma curva bicaracterística. De fato, seja $\alpha$ : $(-\epsilon, \epsilon) \longrightarrow \Omega \times \mathbb{R}^{n}, \epsilon>0$, uma curva bicaracterística para A passando pelo ponto $\left(x_{0}, \xi_{0}\right)$, com $\alpha(0)=\left(x_{0}, \xi_{0}\right)$. Pela definição de $\alpha$ temos

$$
\alpha^{\prime}(s)=\left(\operatorname{grad}_{\xi} A(\alpha(s)),-\operatorname{grad}_{x} A(\alpha(s))\right), \quad s \in(-\epsilon, \epsilon) .
$$

Então,

$$
\begin{aligned}
\frac{d(A \circ \alpha)}{d s}(s) & =\left(\operatorname{grad}_{x} A(\alpha(s)), \operatorname{grad}_{\xi} A(\alpha(s))\right) \cdot \alpha^{\prime}(s) \\
& =\left(\operatorname{grad}_{x} A(\alpha(s)), \operatorname{grad}_{\xi} A(\alpha(s))\right) \cdot\left(\operatorname{grad}_{\xi} A(\alpha(s)),-\operatorname{grad}_{x} A(\alpha(s))\right) \\
& =\sum_{j=1}^{n}\left\{\frac{\partial A}{\partial x_{j}}(\alpha(s)) \frac{\partial A}{\partial \xi_{j}}(\alpha(s))-\frac{\partial A}{\partial \xi_{j}}(\alpha(s)) \frac{\partial A}{\partial x_{j}}(\alpha(s))\right\}=0,
\end{aligned}
$$

para todo $s \in(-\epsilon, \epsilon)$. Logo, $A \circ \alpha$ é constante igual $A\left(x_{0}, \xi_{0}\right)$. 
Definição 3.2.3. Se $A\left(x_{0}, \xi_{0}\right)=0$, a bicaracterística de $A$ que passa por $\left(x_{0}, \xi_{0}\right)$ é chamada de bicaracterística nula.

Pela observação acima, é fácil ver que $A$ é nula ao longo de uma curva bicaracterística nula de $A$.

Definição 3.2.4. Dizemos que $P$ satisfaz a condição $(P)$ se possui a seguinte propriedade:

Para todo $x_{0} \in \Omega, \xi_{0} \in \mathbb{R}^{n} \backslash\{0\}$ e para todo número complexo z tais que

$$
P_{m}\left(x_{0}, \xi_{0}\right)=0, \quad d_{\xi} \Re\left\{z P_{m}\right\}\left(x_{0}, \xi_{0}\right) \neq 0,
$$

a função $\Im\left\{z P_{m}\right\}$, restrita à bicaracterística nula de $\Re\left\{z P_{m}\right\}$ que passa por $\left(x_{0}, \xi_{0}\right)$, não muda de sinal.

Obs. 3.2.5. Vimos na observação 1.3.8 que se $P(x, D)$ é um ODPL de ordem $m$ definido no aberto $\Omega$ de $\mathbb{R}^{n}$ então,

$$
{ }^{t} P_{m}(x, \xi)=(-1)^{m} P_{m}(x, \xi) \text { em } \Omega \times \mathbb{R}^{n} .
$$

Logo é fácil ver que:

$P$ satisfaz a condição $(P)$ se, e somente se, ${ }^{t} P$ satisfaz a condição $(P)$.

Exemplo 3.2.6. Seja $P$ um ODPL definido no aberto $U \subset \mathbb{R}^{2}$ dado por

$$
P(x, D)=D_{x_{2}}+i b\left(x_{1}, x_{2}\right) D_{x_{1}},
$$

onde b é uma função a valores reais. Temos

$$
P_{1}(x, \xi)=\xi_{2}+i b\left(x_{1}, x_{2}\right) \xi_{1}, \quad(x, \xi) \in U \times \mathbb{R}^{2} .
$$

$\operatorname{Sejam}\left(x_{10}, x_{20}\right) \in U$ e $\left(\xi_{10}, \xi_{20}\right) \in \mathbb{R}^{2} \backslash\{0\}$ tais que $P_{1}\left(\left(x_{10}, x_{20}\right),\left(\xi_{10}, \xi_{20}\right)\right)=0$. Então, $\xi_{20}=0, b\left(x_{10}, x_{20}\right)=0$ e $\xi_{10} \in \mathbb{R} \backslash\{0\}$.

Agora, seja $z \in \mathbb{C}$ tal que $d_{\xi} \Re\left\{z P_{1}\left(\left(x_{10}, x_{20}\right),\left(\xi_{10}, \xi_{20}\right)\right)\right\} \neq 0$. Dado $\left(x_{1}, x_{2}\right) \in U$ e $\left(\xi_{1}, \xi_{2}\right) \in \mathbb{R}^{2}$, temos 


$$
z P_{1}\left(\left(x_{1}, x_{2}\right),\left(\xi_{1}, \xi_{2}\right)\right)=\left(\Re\{z\} \xi_{2}-\Im\{z\} \xi_{1} b\left(x_{1}, x_{2}\right)\right)+i\left(\Im\{z\} \xi_{2}+\Re\{z\} \xi_{1} b\left(x_{1}, x_{2}\right) \xi_{1}\right) .
$$

$\log$,

$$
\Re\left\{z P_{1}\left(\left(x_{1}, x_{2}\right),\left(\xi_{1}, \xi_{2}\right)\right)\right\}=\Re\{z\} \xi_{2}-\Im\{z\} \xi_{1} b\left(x_{1}, x_{2}\right)
$$

$e$

$$
\Im\left\{z P_{1}\left(\left(x_{1}, x_{2}\right),\left(\xi_{1}, \xi_{2}\right)\right)\right\}=\Im\{z\} \xi_{2}+\Re\{z\} \xi_{1} b\left(x_{1}, x_{2}\right) .
$$

Assim

$$
d_{\xi} \Re\left\{z P_{1}\left(\left(x_{1}, x_{2}\right),\left(\xi_{1}, \xi_{2}\right)\right)\right\}=-\Im\{z\} b\left(x_{1}, x_{2}\right) d \xi_{1}+\Re\{z\} d \xi_{2} .
$$

$\operatorname{Logo}$,

$$
d_{\xi} \Re\left\{z P_{1}\left(\left(x_{10}, x_{20}\right),\left(\xi_{10}, \xi_{20}\right)\right)\right\}=0+\Re\{z\} d \xi_{2} .
$$

Portanto, $d_{\xi} \Re\left\{z P_{1}\left(\left(x_{10}, x_{20}\right),\left(\xi_{10}, \xi_{20}\right)\right)\right\} \neq 0$ se, e somente se, $z \in\{z \in \mathbb{C} ; \Re\{z\} \neq 0\}$.

Seja $\alpha: I \longrightarrow U \times \mathbb{R}^{2}, \alpha(s)=\left(\left(x_{1}(s), x_{2}(s)\right),\left(\xi_{1}(s), \xi_{2}(s)\right)\right)$, a curva bicaracterística nula de $\Re\left\{z P_{1}\right\}$ que passa por $\left(\left(x_{10}, x_{20}\right),\left(\xi_{10}, \xi_{20}\right)\right)$. Suponha que $0 \in I$ e $\alpha(0)=$ $\left(\left(x_{10}, x_{20}\right),\left(\xi_{10}, \xi_{20}\right)\right)$. Temos que a satisfaz:

1. $x_{1}^{\prime}(s)=-\Im\{z\} b\left(x_{1}(s), x_{2}(s)\right)$;

2. $x_{2}^{\prime}(s)=\Re\{z\}$;

3. $\xi_{1}^{\prime}(s)=\Im\{z\} \xi_{1}(s) \partial_{x_{1}} b\left(x_{1}(s), x_{2}(s)\right)$;

4. $\xi_{2}^{\prime}(s)=\Im\{z\} \xi_{1}(s) \partial_{x_{2}} b\left(x_{1}(s), x_{2}(s)\right)$.

$(s \in I)$

Note que $\xi_{1}(s)=\xi_{1_{0}} e^{f(s)}, s \in I$, onde $f(s)=\Im\{z\} \int_{0}^{s} \partial_{x_{1}} b\left(x_{1}(r), x_{2}(r)\right) d r, s \in I$. Em particular, $\xi_{1}(s) \neq 0, \forall s \in I$, logo $\xi_{1}(s)$ não muda de sinal. 


$$
\left(\Re\left\{z P_{1}\right\} \circ \alpha\right)(s)=\Re\{z\} \xi_{2}(s)-\Im\{z\} \xi_{1}(s) b\left(x_{1}(s), x_{2}(s)\right)=0, \forall s \in I .
$$

$\operatorname{Logo}$,

$$
\xi_{2}(s)=\frac{\Im\{z\}}{\Re\{z\}} \xi_{1}(s) b\left(x_{1}(s), x_{2}(s)\right), \forall s \in I .
$$

Agora vamos analisar $\Im\left\{z P_{1}\right\}$ restrita à curva bicaracterística nula de $\Re\left\{z P_{1}\right\}$ que passa por $\left(x_{10}, x_{20}\right)$.

$$
\begin{aligned}
\left(\Im\left\{z P_{1}\right\} \circ \alpha\right)(s) & =\Im\{z\} \xi_{2}(s)+\Re\{z\} \xi_{1}(s) b\left(x_{1}(s), x_{2}(s)\right) \\
& =\Im\{z\} \frac{\Im\{z\}}{\Re\{z\}} \xi_{1}(s) b\left(x_{1}(s), x_{2}(s)\right)+\Re\{z\} \xi_{1}(s) b\left(x_{1}(s), x_{2}(s)\right) \\
& =\left(\Re\{z\}+\frac{\Im\{z\}^{2}}{\Re\{z\}}\right) \xi_{1}(s) b\left(x_{1}(s), x_{2}(s)\right), \forall s \in I .
\end{aligned}
$$

Ou seja,

$$
\left(\Im\left\{z P_{1}\right\} \circ \alpha\right)(s)=\frac{|z|^{2}}{\Re\{z\}} \xi_{1}(s) b\left(x_{1}(s), x_{2}(s)\right), \forall s \in I .
$$

Note que

$$
\frac{|z|^{2}}{\Re\{z\}} \neq 0, \text { para todo } z \in\{z \in \mathbb{C} ; \Re\{z\} \neq 0\}
$$

Assim, para verificar que $P$ satisfaz a condição $(P)$, basta verificar o sinal de b ao longo de cada curva a como acima. Observe que no caso em que b é uma função que não muda de sinal em $U$, temos que $P$ satisfaz a condição $(P)$ em $U$.

A condição $(P)$ de Nirenberg - Treves é de grande importância na teoria de resolubilidade de operadores diferenciais parciais lineares. O próximo teorema sustenta este comentário.

Teorema 3.2.7. Seja $P$ um ODPL de tipo principal definido em $\Omega$. Então P satisfaz a condição $(P)$ se, e somente se, $P$ é localmente resolúvel.

A suficiência da condição $(P)$ foi demonstrada por R. Beals e C. Fefferman [2] em 1972. Já a necessidade foi demonstrada um pouco mais tarde, no contexto de operadores 
pseudodiferenciais, em 1980 por Moyer [13].

Podemos aplicar o que foi desenvolvido no exemplo 3.2 .6 e o teorema acima para estudar os operadores de Mizohata.

Exemplo 3.2.8. Para cada $k \in \mathbb{N}$, considere o operador

$$
M^{k}(x, D)=D_{x_{2}}-i x_{2}{ }^{k} D_{x_{1}}
$$

definido em $\mathbb{R}^{2}$. Inicialmente, observe que

$$
d_{\xi}\left(M_{1}^{k}\right)(x, \xi)=-i x_{2}{ }^{k} d \xi_{1}+d \xi_{2} \neq 0 \text {, para todo }(x, \xi) \in \mathbb{R}^{2} \times\left(\mathbb{R}^{2} \backslash\{0\}\right) .
$$

Ou seja, $M^{k}$ é de tipo principal.

Quando $k$ é par, temos que $b\left(x_{1}, x_{2}\right) \doteq-x_{2}{ }^{k},\left(x_{1}, x_{2}\right) \in \mathbb{R}^{2}$, não muda de sinal em $\mathbb{R}^{2}$. Assim, pelo exemplo 3.2.6, $M_{k}$ satisfaz a condição (P) em $\mathbb{R}^{2}$. Logo, pelo teorema 3.2.7, $M^{k}$ é localmente resolú vel em $\mathbb{R}^{2}$.

Agora, vamos analisar o caso em que $k$ é impar. Seja $\left(x_{10}, x_{20}\right) \in \mathbb{R}^{2}$, tal que $b\left(x_{10}, x_{20}\right)=-x_{20}{ }^{k}=0$, i.e., $x_{20}=0$ e $x_{10} \in \mathbb{R} . \quad$ Eseja $z \in \mathbb{C}$ tal que $\Re\{z\} \neq 0$. Daí, $\alpha: I \longrightarrow \mathbb{R}^{2}$, com $\alpha(0)=\left(x_{10}, 0\right)$, a curva bicaracterística de $\Re\left\{z M^{k}\right\}$ que passa por $\left(x_{10}, 0\right)$, tem a segunda coordenada dada por

$$
x_{2}(s)=\Re\{z\} s, s \in I .
$$

Assim

$$
b\left(x_{1}(s), x_{2}(s)\right)=-(\Re\{z\} s)^{k}, s \in I .
$$

Logo, $b$ muda de sinal ao longo de $\alpha$. Dai, $\Im\left\{z M^{k}\right\}$ muda de sinal ao longo de $\alpha$. Segue que $M^{k}$ não satisfaz a condição (P) em $\mathbb{R}^{2}$. Assim, pelo teorema 3.2.7, $M^{k}$ não é localmente resolúvel em $\mathbb{R}^{2}$.

Portanto, $M^{k}$, é localmente resolúvel em $\mathbb{R}^{2}$ se, e somente se, $k$ é par. 


\subsection{Resolubilidade no caso hipoelítico}

Nesta parte, vamos estudar a resolubilidade de um ODPL hipoelítico $P$ definido num aberto $\Omega$ do $\mathbb{R}^{n}$. Como foi visto na observação 1.3 .8 o símbolo principal de $P$, quando difere do símbolo de seu transposto, é apenas por um sinal. Como foi visto na seção anterior no teorema 3.2.7, a resolubilidade, no caso de um ODPL de tipo principal, é caracterizada apenas por seu símbolo principal. Assim, o primeiro teorema estabelece a resolubilidade de ${ }^{t} P$. Baseado neste resultado, vamos concluir a resolubilidade de $P$, no caso em que $P$ é de tipo principal.

A técnica usada na demonstração do teorema a seguir é obter através de algumas propriedades de espaços vetoriais topológicos localmente convexos, uma desigualdade "a priori " envolvendo o operador e concluir então a resolubilidade do seu transposto.

Teorema 3.3.1. Se $P(x, D)$ é hipoelítico em $\Omega$, então ${ }^{t} P(x, D)$ é localmente resolúvel em $\Omega$.

Demonstração:(Esta demonstração foi baseada em [27], [32])

Seja $\left(\Omega_{j}\right)_{j \in \mathbb{N}}$ uma sequência de abertos esgotantes de $\Omega$ como no exemplo 1.2.5. Para $j \in \mathbb{N}$ e $m \in \mathbb{Z}_{+}$definimos

$$
P_{j, m}(u) \doteq\|u\|_{L^{2}\left(\Omega_{j}\right)}+\sum_{|\alpha| \leq m} \sup _{\Omega_{j}}\left|\partial^{\alpha} P(x, D) u\right|, u \in C^{\infty}(\Omega) .
$$

Seja $\mathscr{P}=\left\{P_{j, m} ; j \in \mathbb{N}\right.$ e $\left.m \in \mathbb{Z}_{+}\right\}$.

Seja E o ELC constituído do espaço vetorial $C^{\infty}(\Omega)$ com a topologia gerada pela família de seminormas $\mathscr{P}$. Mais que um ELC, E é Fréchet. De fato, seja $\left(u_{l}\right)_{l \in \mathbb{N}}$ uma sequência de Cauchy em E. Para cada $j \in \mathbb{N}$ e $m \in \mathbb{Z}_{+}$temos

$$
P_{j, m}\left(u_{l}-u_{n}\right) \longrightarrow 0 \text { quando } l, n \longrightarrow \infty \text {. }
$$

Como 


$$
\sum_{|\alpha| \leq m} \sup _{\Omega_{j}}\left|\partial^{\alpha} P(x, D) u\right| \leq P_{j, m}(u)
$$

para toda $u \in C^{\infty}(\Omega)$, temos

$$
\sum_{|\alpha| \leq m} \sup _{\Omega_{j}}\left|\partial^{\alpha} P(x, D)\left(u_{l}-u_{n}\right)\right| \longrightarrow 0 \text { quando } l, n \longrightarrow \infty \text {. }
$$

Portanto, $\left(P(x, D) u_{l}\right)_{l \in \mathbb{N}}$ é uma sequência de Cauchy em $C^{\infty}(\Omega)$. Por outro lado, dado $K \subset \Omega$ compacto, existe $j_{0} \in \mathbb{N}$ tal que $K \subset \Omega_{j_{0}}$. Como

$$
P_{j_{0}, m}\left(u_{l}-u_{n}\right) \longrightarrow 0 \text { quando } l, n \longrightarrow \infty
$$

e

$$
\|u\|_{L^{2}(K)} \leq\|u\|_{L^{2}\left(\Omega_{j_{0}}\right)} \leq P_{j_{0}, m}(u)
$$

para toda $u \in C^{\infty}(\Omega)$, temos

$$
\left\|u_{l}-u_{n}\right\|_{L^{2}(K)} \longrightarrow 0 \text { quando } l, n \longrightarrow \infty \text {. }
$$

Portanto, $\left(u_{l}\right)_{l \in \mathbb{N}}$ é uma sequência de Cauchy em $L_{l o c}^{2}(\Omega)$.

Assim, existe $u \in L_{l o c}^{2}(\Omega)$ e $f \in C^{\infty}(\Omega)$ tais que

$$
u_{l} \longrightarrow u \text { em } L_{l o c}^{2}(\Omega) \quad \text { e } \quad P(x, D) u_{l} \longrightarrow f \text { em } C^{\infty}(\Omega)
$$

Logo,

$$
u_{l} \longrightarrow u \quad \text { e } \quad P(x, D) u_{l} \longrightarrow f \text { em } \mathscr{D}^{\prime}(\Omega)
$$

Por outro lado, como $P(x, D): \mathscr{D}^{\prime}(\Omega) \longrightarrow \mathscr{D}^{\prime}(\Omega)$ é contínuo, $P(x, D) u_{l} \longrightarrow P(x, D) u$ em $\mathscr{D}^{\prime}(\Omega)$, consequentemente $P(x, D) u=f$ em $\mathscr{D}^{\prime}(\Omega)$. Daí, como $P$ é hipoelítico e $f \in C^{\infty}(\Omega)$ temos que $u \in C^{\infty}(\Omega)$. Vamos mostrar que $u_{l} \longrightarrow u$ em E. Sejam $j \in \mathbb{N}$ e $m \in \mathbb{Z}_{+}$, temos

- $\left\|u_{l}-u\right\|_{L^{2}\left(\Omega_{j}\right)} \leq\left\|u_{l}-u\right\|_{L^{2}\left(\overline{\Omega_{j}}\right)} \longrightarrow 0$, pois $u_{l} \longrightarrow u$ em $L_{l o c}^{2}(\Omega)$.

- $\sum_{|\alpha| \leq m} \sup _{\Omega_{j}}\left|\partial^{\alpha} P(x, D)\left(u_{l}-u\right)\right|=\sum_{|\alpha| \leq m} \sup _{\Omega_{j}}\left|\partial^{\alpha}\left(P(x, D) u_{l}-P(x, D) u\right)\right|$ 
$=\sum_{|\alpha| \leq m} \sup _{\Omega_{j}}\left|\partial^{\alpha}\left(P(x, D) u_{l}-f\right)\right| \longrightarrow 0$, pois $P(x, D) u_{l} \longrightarrow f$ em $C^{\infty}(\Omega)$.

Portanto,

$$
P_{j, m}\left(u_{l}-u\right)=|| u_{l}-u \|_{L^{2}\left(\Omega_{j}\right)}+\sum_{|\alpha| \leq m} \sup _{\Omega_{j}}\left|\partial^{\alpha} P(x, D)\left(u_{l}-u\right)\right| \longrightarrow 0 .
$$

Assim, temos que $\left(u_{l}\right)_{l \in \mathbb{N}}$ é convergente em E. Portanto, E é Fréchet.

Dados $\alpha \in \mathbb{Z}_{+}^{n}$ e $j \in \mathbb{N}$, segue da regra de Leibniz que existem constantes positivas $C_{\alpha, j}$ e $N_{\alpha, j}$ tais que

$$
\sup _{\Omega_{j}}\left|\partial^{\alpha}(P(x, D) u)\right| \leq C_{\alpha, j} \sum_{|\sigma| \leq N_{\alpha, j}} \sup _{\Omega_{j}}\left|\partial^{\sigma} u\right|
$$

Assim, existem constantes positivas $M$ e $C$ (que dependem de $j$ ) tais que

$$
\sum_{|\alpha| \leq m} \sup _{\Omega_{j}}\left|\partial^{\alpha}(P(x, D) u)\right| \leq C \sum_{|\eta| \leq M} \sup _{\Omega_{j}}\left|\partial^{\eta} u\right| .
$$

Por outro lado,

$$
\|u\|_{L^{2}\left(\Omega_{j}\right)}=\left(\int_{\Omega_{j}}|u|^{2} d x\right)^{\frac{1}{2}} \leq \mathrm{m}\left(\Omega_{j}\right)^{\frac{1}{2}} \sup _{\Omega_{j}}|u|
$$

Onde $\mathrm{m}$ denota a medida de Lebesgue em $\mathbb{R}^{n}$.

Assim

$$
\begin{gathered}
\|u\|_{L^{2}\left(\Omega_{j}\right)}+\sum_{|\alpha| \leq m} \sup _{\Omega_{j}}\left|\partial^{\alpha}(P(x, D) u)\right| \leq \mathrm{m}\left(\Omega_{j}\right)^{\frac{1}{2}} \sup _{\Omega_{j}}|u|+C \sum_{|\eta| \leq M} \sup _{\Omega_{j}}\left|\partial^{\eta} u\right| \\
\leq C_{j} \sum_{|\eta| \leq k} \sup _{\Omega_{j}}\left|\partial^{\eta} u\right| .
\end{gathered}
$$

Ou seja,

$$
P_{j, m}(u) \leq C_{j} p_{k, j}(u),
$$


onde $p_{k, j}$ é uma seminorma que pertence a família de seminormas que geram a topologia de $C^{\infty}(\Omega)$. Pela arbitrariedade de $j \in \mathbb{N}$ e $m \in \mathbb{Z}_{+}$, temos

$$
\begin{aligned}
I d: C^{\infty}(\Omega) & \longrightarrow \mathbf{E} \\
u & \longmapsto u
\end{aligned}
$$

é contínua (proposição 1.2.13). Como Id é uma bijeção entre os espaços de Fréchet $C^{\infty}(\Omega)$ e E, pelo teorema da Aplicação Aberta (teorema 1.2.9)

$$
\begin{aligned}
I d: \mathbf{E} & \longrightarrow C^{\infty}(\Omega) \\
u & \longmapsto u
\end{aligned}
$$

é contínua. Assim, pela proposição 1.2 .13 temos que, para todo $l \in \mathbb{N}$ e $k \in \mathbb{Z}_{+}$, existem $j \in \mathbb{N}$ e $m \in \mathbb{Z}_{+}$e uma constante $C=C(l, k)$ tais que:

$$
p_{l, k}(u) \leq C P_{j, m}(u)
$$

para toda $u \in \mathbf{E}$. Isto é,

$$
\sum_{|\eta| \leq k} \sup _{\Omega_{l}}\left|\partial^{\eta} u\right| \leq C\left(\|u\|_{L^{2}\left(\Omega_{j}\right)}+\sum_{|\alpha| \leq m} \sup _{\Omega_{j}}\left|\partial^{\alpha}(P(x, D) u)\right|\right),
$$

para toda $u \in C^{\infty}(\Omega)$. Seja dado $x_{0} \in \Omega$. Existe $j \in \mathbb{N}$ tal que $x_{0} \in \Omega_{j-1}$. Assim, para $k=0, l=j$ e $m \in \mathbb{Z}_{+}$temos

$$
\sup _{\Omega_{j}}|u| \leq C\left(\|u\|_{L^{2}\left(\Omega_{j}\right)}+\sum_{|\alpha| \leq m} \sup _{\Omega_{j}}\left|\partial^{\alpha}(P(x, D) u)\right|\right), \quad \forall u \in C^{\infty}(\Omega) .
$$

Lembrando que $C=C(l, k)$ e $\Omega_{j-1} \subset \overline{\Omega_{j-1}} \subset \Omega_{j}$, com $\overline{\Omega_{j-1}}$ compacto. Daí, segue que se $U \subset \Omega_{j-1}$ é uma vizinhança aberta de $x_{0}$ com

$$
m(U) \leq \frac{1}{4 C^{2}}
$$

temos que para $u \in C_{c}^{\infty}(U)$, são satisfeitas:

1. $\sup _{U}|u|=\sup _{\Omega_{j-1}}|u| \leq \sup _{\Omega_{j}}|u|$

2. $\|u\|_{L^{2}\left(\Omega_{j}\right)} \leq\left(\int_{\Omega_{j}}|u|^{2} d x\right)^{\frac{1}{2}}=\left(\int_{U}|u|^{2} d x\right)^{\frac{1}{2}} \leq\left(\sup _{U}|u|\right)(m(U))^{\frac{1}{2}} \leq \frac{1}{2 C} \sup _{U}|u|$. 
Aplicando 1 e 2 em (3.3.1) temos

$$
\begin{aligned}
\sup _{U}|u| & \leq C\left(\frac{1}{2 C} \sup _{U}|u|+\sum_{|\alpha| \leq m} \sup _{\Omega_{j}}\left|\partial^{\alpha}(P(x, D) u)\right|\right) \\
& \leq \frac{1}{2} \sup _{U}|u|+C \sum_{|\alpha| \leq m} \sup _{\Omega_{j}}\left|\partial^{\alpha}(P(x, D) u)\right| \\
& =\frac{1}{2} \sup _{U}|u|+C \sum_{|\alpha| \leq m} \sup _{U}\left|\partial^{\alpha}(P(x, D) u)\right|
\end{aligned}
$$

para toda $u \in C_{c}^{\infty}(U)$. Daí,

$$
\sup _{U}|u| \leq 2 C \sum_{|\alpha| \leq m} \sup _{U}\left|\partial^{\alpha}(P(x, D) u)\right|, \quad \forall u \in C_{c}^{\infty}(U) .
$$

Considere o espaço $C_{c}^{m}(U)$ com a norma

$$
\|w\|=\sum_{|\alpha| \leq m} \sup _{U}\left|\partial^{\alpha} w\right|, w \in C_{c}^{m}(U)
$$

Temos que

$$
\mathbf{F} \doteq\left\{P(x, D) u ; u \in C_{c}^{\infty}(U)\right\} \subset C_{c}^{m}(U)
$$

é um subespaço vetorial de $C_{c}^{m}(U)$. Dada $f \in C_{c}^{\infty}(U)$, definimos o funcional linear

$$
T_{f}: \mathbf{F} \longrightarrow \mathbb{C}
$$

por

$$
\left\langle T_{f}, P(x, D) v\right\rangle=\int_{U} f v d x, P(x, D) v \in \mathbf{F}
$$

O funcional $T_{f}$ está bem definido. Temos também que $T_{f}$ é contínuo. Segue direto de 
(3.3.2). De fato, seja $w \in \mathbf{F}$. Temos que existe $v \in C_{c}^{\infty}(U)$ tal que $P(x, D) v=w$. Assim

$$
\begin{aligned}
\left|\left\langle T_{f}, w\right\rangle\right| & =\left|\left\langle T_{f}, P(x, D) v\right\rangle\right| \leq \int_{U}|f v| d x \leq \sup _{U}|v|\left(\int_{U}|f| d x\right) \\
& \leq \tilde{C} \sum_{|\alpha| \leq m} \sup _{U}\left|\partial^{\alpha}(P(x, D) v)\right|=\tilde{C} \sum_{|\alpha| \leq m} \sup _{U}\left|\partial^{\alpha} w\right| \\
& =\tilde{C}|| w|| .
\end{aligned}
$$

Pelo teorema de Hahn-Banach, existe $\tilde{T}_{f}: C_{c}^{m}(U) \longrightarrow \mathbb{C}$ funcional linear que estende $T_{f}$, isto é, $\left.\tilde{T}_{f}\right|_{\mathbf{F}}=T_{f}$, e satisfaz

$$
\left|\left\langle\tilde{T}_{f}, w\right\rangle\right| \leq \tilde{C}\|w\| \text {, para toda } w \in C_{c}^{m}(U)
$$

Ou seja,

$$
\left|\left\langle\tilde{T}_{f}, w\right\rangle\right| \leq \tilde{C} \sum_{|\alpha| \leq m} \sup _{U}\left|\partial^{\alpha} w\right|
$$

para toda $w \in C_{c}^{m}(U)$. Em particular, se $K \subset U$ é um compacto, temos

$$
\left|\left\langle\tilde{T}_{f}, \varphi\right\rangle\right| \leq \tilde{C} \sum_{|\alpha| \leq m} \sup _{U}\left|\partial^{\alpha} \varphi\right|=\tilde{C} \sum_{|\alpha| \leq m} \sup _{K}\left|\partial^{\alpha} \varphi\right|
$$

para toda $\varphi \in C_{c}^{\infty}(K)$. Segue daí que $\tilde{T}_{f} \in \mathscr{D}^{\prime}(U)$. Mais que uma distribuição em $U, \tilde{T}_{f}$ satisfaz

$$
{ }^{t} P(x, D) \tilde{T}_{f}=f \text { em } \mathscr{D}^{\prime}(U) .
$$

De fato, basta notar que se $\varphi \in C_{c}^{\infty}(U)$,

$$
\left\langle{ }^{t} P(x, D) \tilde{T}_{f}, \varphi\right\rangle=\left\langle\tilde{T}_{f}, P(x, D) \varphi\right\rangle=\left\langle T_{f}, P(x, D) \varphi\right\rangle=\int_{U} f \varphi d x=\langle f, \varphi\rangle .
$$

Corolário 3.3.2. Se $P(x, D)$ é hipoelítico em $\Omega \subset \mathbb{R}^{2}$, e tem a forma

$$
P=a_{1} D_{x_{1}}+a_{2} D_{x_{2}} \operatorname{com} D_{x_{1}}\left(a_{1}\right)+D_{x_{2}}\left(a_{2}\right)=0 \text { em } \Omega,
$$

então $P$ é localmente resolúvel. 
Demonstração: O resultado segue direto do teorema 3.3.1 observando que

$$
P^{t}=-P-\left(D_{x_{1}}\left(a_{1}\right)+D_{x_{2}}\left(a_{2}\right)\right)=-P, \text { em } \Omega .
$$

Corolário 3.3.3. Se $P(x, D)$ é de tipo principal e hipoelítico, então $P(x, D)$ é localmente resolúvel.

\section{Demonstração:}

Pelo teorema 3.3.1, ${ }^{t} P(x, D)$ é localmente resolúvel em $\Omega$. Logo, pelo teorema 3.2.7, ${ }^{t} P(x, D)$ satisfaz a condição $(\mathrm{P})$. Da observação 3.2.5, temos que $P(x, D)$ satisfaz a condição $(\mathrm{P})$ e novamente, pelo teorema 3.2.7 temos que $P(x, D)$ é localmente resolúvel.

Nem todo operador hipoelítico é localmente resolúvel.

Exemplo 3.3.4. Considere o operador

$$
P(x, D)=D_{x_{1}}+i x_{1} D_{x_{2}}^{2}
$$

em $\mathbb{R}^{2}$. O símbolo principal de $P$ é $P_{2}(x, \xi)=i x_{1} \xi_{2}{ }^{2}$. E assim $d_{\xi} P_{2}(x, \xi)=2 i x_{1} \xi_{2} d \xi_{2}$. Logo P não é de tipo principal. Kannai [15] mostrou que $P$ é hipoelítico em $\mathbb{R}^{2}$, mas não é localmente resolúvel em nenhum aberto que contenha pontos da reta $\left\{\left(x_{1}, x_{2}\right) ; x_{1}=0\right\}$.

Os operadores de tipo principal hipoelíticos possuem uma propriedade extra e automática em relação à regularidade de suas soluções. Eles são localmente $C^{\infty}$ resolúveis.

Definição 3.3.5. $P(x, D)$ é dito localmente $C^{\infty}$ resolúvel em $\Omega$ quando $\forall x_{0} \in \Omega$ existe um aberto $U \subset \Omega$, com $x_{0} \in U$, de modo que $\forall f \in C_{c}^{\infty}(U)$ existe $u \in C^{\infty}(\Omega)$ com $P(x, D) u=f$ em $U$.

Corolário 3.3.6. Se $P(x, D)$ é de tipo principal e hipoelítico, então $P(x, D)$ é localmente $C^{\infty}$ resolúvel.

\section{Demonstração:}


Seja $x_{0} \in \Omega$. Pelo corolário acima $P$ é localmente resolúvel, $\log$ o existe um aberto $U \subset \Omega$, com $x_{0} \in U$ tal que para cada $f \in C_{c}^{\infty}(U)$ existe $u \in \mathscr{D}^{\prime}(\Omega)$ de modo que $P(x, D) u=f$ em $U$. Como $P$ é hipoelítico, se $u \in \mathscr{D}^{\prime}(\Omega)$ é tal que $P(x, D) u=f \operatorname{com} f \in C^{\infty}(U)$, então, $u \in C^{\infty}(U)$. Portanto, para toda $f \in C_{c}^{\infty}(U)$ existe $u \in C^{\infty}(U)$ de modo que $P(x, D) u=f$ em $U$.

\subsection{A Condição (Q)}

A condição (Q), de modo semelhante à condição (P), é baseada no estudo do sinal, mais precisamente, no anulamento da parte imaginária (do símbolo principal) de um ODPL restrita a curvas bicaracterísticas da parte real. A condição (Q) desempenha um papel importante no estudo da hipoeliticidade de um ODPL de tipo principal.

O próximo exemplo mostra que existe uma relação estreita entre a hipoeliticidade e o anulamento, ao longo de certas curvas, de coeficientes de um campo vetorial complexo no plano.

Exemplo 3.4.1. Seja $L$ um campo vetorial complexo definido em um aberto $\Omega$ de $\mathbb{R}^{2}$. Suponha L escrito na forma

$$
L=A \frac{\partial}{\partial x_{1}}+B \frac{\partial}{\partial x_{2}} e m \Omega
$$

Suponha também que existe uma curva diferenciável $\left(C^{\infty}\right) \alpha \doteq\left(\alpha_{1}, \alpha_{2}\right):(-1,1) \longrightarrow \Omega$, tal que $\alpha_{2}^{\prime}(s) \neq 0$ e $(A \circ \alpha)(s)=0, \forall s \in(-1,1)$. Em particular $\alpha_{2}:(-1,1) \longrightarrow \mathbb{R} e ́$ injetiva.

Então L não é hipoelítico em $\Omega$. De fato, defina

$$
F\left(y_{1}, y_{2}\right)=\left(\alpha_{1}\left(y_{1}\right)+y_{2}, \alpha_{2}\left(y_{1}\right)\right),\left(y_{1}, y_{2}\right) \in(-\delta, \delta) \times(-\delta, \delta), 0<\delta<1 .
$$

$\operatorname{Sejam}\left(y_{1}, y_{2}\right),\left(\tilde{y}_{1}, \tilde{y}_{2}\right) \in(-\delta, \delta) \times(-\delta, \delta)$. Se $F\left(y_{1}, y_{2}\right)=F\left(\tilde{y}_{1}, \tilde{y}_{2}\right)$, então

$$
\alpha_{1}\left(y_{1}\right)+y_{2}=\alpha_{1}\left(\tilde{y}_{1}\right)+\tilde{y}_{2} \text { e } \alpha_{2}\left(y_{1}\right)=\alpha_{2}\left(\tilde{y}_{1}\right)
$$


Logo, $y_{1}=\tilde{y}_{1}$, pois $\alpha_{2}$ é injetiva. Dai, $\alpha_{1}\left(y_{1}\right)+y_{2}=\alpha_{1}\left(y_{1}\right)+\tilde{y}_{2}$. Logo, $y_{2}=\tilde{y}_{2}$. Portanto, $\left(y_{1}, y_{2}\right)=\left(\tilde{y}_{1}, \tilde{y}_{2}\right)$. Ou seja, F é injetiva.

Agora observe que

$$
J(F)\left(y_{1}, y_{2}\right)=\left[\begin{array}{cc}
\alpha_{1}^{\prime}\left(y_{1}\right) & 1 \\
\alpha_{2}^{\prime}\left(y_{1}\right) & 0
\end{array}\right]
$$

para $\left(y_{1}, y_{2}\right) \in(-\delta, \delta) \times(-\delta, \delta) . \quad$ Logo, $\operatorname{det}\left(J(F)\left(y_{1}, y_{2}\right)\right)=-\alpha_{2}^{\prime}\left(y_{1}\right) \neq 0, \forall\left(y_{1}, y_{2}\right) \in$ $(-\delta, \delta) \times(-\delta, \delta)$. Assim, reduzindo $\delta$ se necessário, obtemos que $F$ é um difeomorfismo de $(-\delta, \delta) \times(-\delta, \delta)$ sobre $F((-\delta, \delta) \times(-\delta, \delta)) \subset \Omega$.

\section{Temos}

$$
L_{F^{-1}}=\left(L\left(F_{1}\right) \circ F^{-1}\right) \frac{\partial}{\partial y_{1}}+\left(L\left(F_{2}\right) \circ F^{-1}\right) \frac{\partial}{\partial y_{2}}
$$

$e m(-\delta, \delta) \times(-\delta, \delta)$. Sejam

$$
A_{F} \doteq\left(L\left(F_{1}\right) \circ F^{-1}\right) \text { e } B_{F} \doteq\left(L\left(F_{2}\right) \circ F^{-1}\right)
$$

Note que $B_{F}=\left(L\left(F_{2}\right) \circ F^{-1}\right)=\left(A \circ F^{-1}\right) \cdot\left(\alpha_{2}^{\prime}\left(\pi \circ F^{-1}\right)\right)$, em particular,

$$
B_{F}\left(y_{1}, 0\right)=0 \text {, para todo } y_{1} \in(-\delta, \delta) \text {. }
$$

Seja $u\left(y_{1}, y_{2}\right)=H\left(y_{2}\right),\left(y_{1}, y_{2}\right) \in(-\delta, \delta) \times(-\delta, \delta)$ onde

$$
H\left(y_{2}\right)=\left\{\begin{array}{lll}
1, & \text { se } & y_{2} \in(-\delta, 0) \\
0, & \text { se } & y_{2} \in[0, \delta)
\end{array}\right.
$$

para $y_{2} \in(-\delta, \delta)$

Afirmamos que $L_{F^{-1}} u=0$ em $(-\delta, \delta) \times(-\delta, \delta)$.

De fato, seja $\varphi \in C_{c}^{\infty}((-\delta, \delta) \times(-\delta, \delta))$. Temos

$$
\left\langle L_{F^{-1}} u, \varphi\right\rangle=\left\langle A_{F} \frac{\partial u}{\partial y_{1}}+B_{F} \frac{\partial u}{\partial y_{2}}, \varphi\right\rangle=\left\langle A_{F} \frac{\partial u}{\partial y_{1}}, \varphi\right\rangle+\left\langle B_{F} \frac{\partial u}{\partial y_{2}}, \varphi\right\rangle .
$$


Logo,

$$
\left\langle L_{F^{-1}} u, \varphi\right\rangle=-\left\langle u, \frac{\partial\left(A_{F} \varphi\right)}{\partial y_{1}}\right\rangle-\left\langle u, \frac{\partial\left(B_{F} \varphi\right)}{\partial y_{2}}, \varphi\right\rangle
$$

Vamos analisar cada parcela da igualdade acima separadamente. Como $\varphi$ tem suporte compacto, existe $a>0$ tal que $\operatorname{supp}(\varphi) \subset(-a, a) \times(-a, a) \subset(-\delta, \delta) \times(-\delta, \delta)$.

$$
\begin{aligned}
& \text { - }\left\langle u, \frac{\partial\left(A_{F} \varphi\right)}{\partial y_{1}}\right\rangle=\int_{-\delta}^{\delta} \int_{-\delta}^{\delta} u\left(y_{1}, y_{2}\right) \frac{\partial\left(A_{F} \varphi\right)}{\partial y_{1}}\left(y_{1}, y_{2}\right) d y_{1} d y_{2}= \\
& =\int_{-a}^{a} \int_{-a}^{a} u\left(y_{1}, y_{2}\right) \frac{\partial\left(A_{F} \varphi\right)}{\partial y_{1}}\left(y_{1}, y_{2}\right) d y_{1} d y_{2}=\int_{-a}^{a} H\left(y_{2}\right) \int_{-a}^{a} \frac{\partial\left(A_{F} \varphi\right)}{\partial y_{1}}\left(y_{1}, y_{2}\right) d y_{1} d y_{2} \\
& =\int_{-a}^{a} H\left(y_{2}\right)\left[\left(A_{F} \varphi\right)\left(a, y_{2}\right)-\left(A_{F} \varphi\right)\left(-a, y_{2}\right)\right] d y_{2} \\
& =\int_{-a}^{a} H\left(y_{2}\right)\left[A_{F}\left(a, y_{2}\right) \varphi\left(a, y_{2}\right)-A_{F}\left(-a, y_{2}\right) \varphi\left(-a, y_{2}\right)\right] d y_{2} \\
& =\int_{-a}^{a} H\left(y_{2}\right)\left[A_{F}\left(a, y_{2}\right) \cdot 0-A_{F}\left(-a, y_{2}\right) \cdot 0\right] d y_{2}=\int_{-a}^{a} 0 d y_{2}=0 . \\
& \text { - }\left\langle u, \frac{\partial\left(B_{F} \varphi\right)}{\partial y_{2}}\right\rangle=\int_{-\delta}^{\delta} \int_{-\delta}^{\delta} u\left(y_{1}, y_{2}\right) \frac{\partial\left(B_{F} \varphi\right)}{\partial y_{2}}\left(y_{1}, y_{2}\right) d y_{1} d y_{2}= \\
& =\int_{-\delta}^{\delta} \int_{-\delta}^{\delta} u\left(y_{1}, y_{2}\right) \frac{\partial\left(B_{F} \varphi\right)}{\partial y_{2}}\left(y_{1}, y_{2}\right) d y_{2} d y_{1} \\
& =\int_{-a}^{a} \int_{-a}^{a} H\left(y_{2}\right) \frac{\partial\left(B_{F} \varphi\right)}{\partial y_{2}}\left(y_{1}, y_{2}\right) d y_{2} d y_{1}=\int_{-a}^{a} \int_{-a}^{0} \frac{\partial(B \varphi)}{\partial y_{2}}\left(y_{1}, y_{2}\right) d y_{2} d y_{1} \\
& =\int_{-a}^{a}\left[\left(B_{F} \varphi\right)\left(y_{1}, 0\right)-\left(B_{F} \varphi\right)\left(y_{1},-a\right)\right] d y_{2} \\
& =\int_{-a}^{a}\left[B_{F}\left(y_{1}, 0\right) \varphi\left(y_{1}, 0\right)-B_{F}\left(y_{1},-a\right) \varphi\left(y_{1},-a\right)\right] d y_{2} \\
& =\int_{-a}^{a}\left[0 \cdot \varphi\left(y_{1}, 0\right)-B_{F}\left(y_{1},-a\right) \cdot 0\right] d y_{2}=\int_{-a}^{a} 0 d y_{2}=0 .
\end{aligned}
$$

Assim temos $\left\langle L_{F^{-1}} u, \varphi\right\rangle=0$. Pela arbitrariedade de $\varphi \in C_{c}^{\infty}(\Omega)$, temos $L_{F^{-1}} u=0$.

Portanto $L_{F^{-1}}$ não é hipoelítico, pois $L_{F^{-1}} u=0 \in C^{\infty}(F((-\delta, \delta) \times(-\delta, \delta)))$, mas $u \notin C^{\infty}(F((-\delta, \delta) \times(-\delta, \delta)))$. Concluímos assim que $L$ não é hipoelítico, uma vez que hipoeliticidade é invariante por mudança de coordenadas. 
Obs. 3.4.2. Segue do exemplo acima que se $L=A \frac{\partial}{\partial x_{1}}+B \frac{\partial}{\partial x_{2}}$ é um campo vetotial complexo em $\Omega$, com

$$
(A \circ \alpha)(s)=(B \circ \alpha)(s)=0, \text { para todo } s \in(-1,1) \text {, }
$$

onde $\alpha:(-1,1) \longrightarrow \Omega$ uma curva diferenciável $\left(C^{\infty}\right)$ com $\alpha^{\prime}(s) \neq 0$, para todo $s \in$ $(-1,1)$, então L não é hipoelítico. Em resumo, se um campo é singular em cada ponto de uma curva então ele não pode ser hipoelítico.

Agora vamos definir a condição (Q).

Definição 3.4.3. Dizemos que $P$ satisfaz a condição $(Q)$ se possui a seguinte propriedade:

Para todo $x_{0} \in \Omega, \xi_{0} \in \mathbb{R}^{n} \backslash\{0\}$ e para todo número complexo z tais que

$$
P_{m}\left(x_{0}, \xi_{0}\right)=0, \quad d_{\xi} \Re\left\{z P_{m}\right\}\left(x_{0}, \xi_{0}\right) \neq 0,
$$

a função $\Im\left\{z P_{m}\right\}$ é não identicamente nula em toda vizinhança de $\left(x_{0}, \xi_{0}\right)$ na bicaracterística de $\Re\left\{z P_{m}\right\}$ que passa por $\left(x_{0}, \xi_{0}\right)$.

É fácil ver que se $P$ é um ODPL elítico, então $P$ satisfaz a condição (Q).

Exemplo 3.4.4. Os operadores de Mizohata definidos no exemplo 3.2.8, i.e., para $k \in \mathbb{N}$,

$$
M^{k}(x, D)=D_{x_{2}}-i x_{2}{ }^{k} D_{x_{1}}
$$

em $\mathbb{R}^{2}$, satisfazem a condição $(Q)$ em $\mathbb{R}^{2}$. De fato, vamos aplicar o exemplo 3.2.6. Assim na mesma notação do exemplo 3.2.6, seja $b\left(x_{1}, x_{2}\right) \doteq-x_{2}{ }^{k},\left(x_{1}, x_{2}\right) \in \mathbb{R}^{2}$. Seja $\left(x_{10}, x_{20}\right) \in \mathbb{R}^{2}$, tal que $b\left(x_{10}, x_{20}\right)=-x_{20}{ }^{k}=0$, i.e., $x_{20}=0$ e $x_{10} \in \mathbb{R}$. E seja $z \in \mathbb{C}$ tal que $\Re\{z\} \neq 0$. Daí, $\alpha: I \longrightarrow \mathbb{R}^{2}$, com $\alpha(0)=\left(x_{10}, 0\right)$, a curva bicaracterística de $\Re\left\{z M^{k}\right\}$ que passa por $\left(x_{11}, 0\right)$, tem a segunda coordenada dada por

$$
x_{2}(s)=\Re\{z\} s, s \in I .
$$

Assim

$$
b\left(x_{1}(s), x_{2}(s)\right)=-(\Re\{z\} s)^{k}, s \in I .
$$


Logo, ao longo da curva $\alpha, b$ se anula apenas no ponto $\alpha(0)=\left(x_{10}, 0\right)$. Daí, $\Im\left\{z M^{k}\right\}$ se anula apenas no ponto $\alpha(0)=\left(x_{10}, 0\right)$. Segue dai, que $M^{k}$ satisfaz a condição $(Q)$.

Obs. 3.4.5. (Análoga à observação 3.2.5) se $P(x, D)$ é um ODPL de ordem $m$ definido no aberto $\Omega$ de $\mathbb{R}^{n}$ então,

$$
{ }^{t} P_{m}(x, \xi)=(-1)^{m} P_{m}(x, \xi) e m \Omega \times \mathbb{R}^{n}
$$

Logo é fácil ver que:

$$
P \text { satisfaz a condição (Q) se, e somente se, }{ }^{t} P \text { satisfaz a condição }(Q) \text {. }
$$

Agora vamos discutir uma caracterização de ODPL de tipo principal hipoelíticos em função do símbolo principal. Em [29] podemos encontrar os dois seguintes teoremas:

Teorema 3.4.6. Seja $P(x, D)$ um ODPL em $\Omega$. Se P é hipoelítico em $\Omega$, então $P(x, D)$ satisfaz a condição (Q) em $\Omega$.

Este teorema pode ser pensado como uma generalização do exemplo 3.4.1.

Teorema 3.4.7. Seja $P(x, D)$ um $\boldsymbol{O D P L}$ de tipo principal em $\Omega$. Se P satisfaz as condições $(P)$ e $(Q)$ em $\Omega$, então $P$ é hipoelítico em $\Omega$.

Pelos dois teoremas acima e dos resultados dos capítulos precedentes, temos

Teorema 3.4.8. Seja $P(x, D)$ um $\boldsymbol{O D P L}$ de tipo principal em $\Omega$. P é hipoelítico em $\Omega$ se, e somente se, $P$ satisfaz as condições $(P)$ e $(Q)$ em $\Omega$.

\section{Demonstração:}

Pelo corolário 3.3.3 temos que $P$ é localmente resolúvel em $\Omega$. Logo, pelo teorema 3.2.7 temos que $P$ satisfaz a condição $(\mathrm{P})$ em $\Omega$. E assim a conclusão segue direto dos teoremas 3.4.6 e 3.4.7.

Obs. 3.4.9. Em [29] o teorema 3.4.8 é uma conjectura, devido ao fato que não se sabia que a condição $(P)$ era necessária para a resolubilidade local. 
Como as condições (P) e (Q) são estabelecidas em função do símbolo principal do operador, o estudo da hipoeliticidade de um operador de tipo principal se restringe aos termos do operador que possuem as derivadas parciais com maior grau, i.e., se

$$
P(x, D)=\sum_{|\alpha| \leq m} a_{\alpha}(x) D^{\alpha}
$$

é de tipo principal então $P$ é hipoelítico, se e somente se,

$$
P_{m}(x, D)=\sum_{|\alpha|=m} a_{\alpha}(x) D^{\alpha}
$$

é hipoelítico.

Exemplo 3.4.10. Vimos nos exemplos 3.2.8 e 3.4.4 que os operadores $M^{k}$ satisfazem as condições $(P)$ e $(Q)$ em $\mathbb{R}^{2}$ quando $k \in \mathbb{N}$ é par. Assim, pelo teorema 3.4 .8 os operadores $M^{k}$, com $k \in \mathbb{N}$ par, são hipoelíticos em $\mathbb{R}^{2}$.

Obs. 3.4.11. Os operadores $M^{k}$, com $k \in \mathbb{N}$ par, são exemplos de operadores hipoelíticos que não são elíticos. 


\subsection{Integrabilidade Local no Plano}

Seja $L$ um campo vetorial complexo definido no aberto $\Omega \subset \mathbb{R}^{2}$.

Proposição 3.5.1. Se L é não singular e localmente $C^{\infty}$ resolúvel, então L é localmente integrável.

Demonstração: (Esta demonstração foi baseada em [31]). Inicialmente, observe que olhando para $L$ como um operador diferencial parcial, temos que

$$
{ }^{t} L(x, D)=-L(x, D)+i c(x)
$$

em $\Omega$, onde $c=\left(\partial_{x_{1}} L\left(\pi_{1}\right)+\partial_{x_{2}} L\left(\pi_{2}\right)\right) \in C^{\infty}(\Omega)$.

Seja $x_{0} \in \Omega$. Por hipótese existe uma vizinhança aberta $V_{0}$ de $x_{0}$ em $\Omega$ e uma função $v \in C^{\infty}\left(V_{0}\right)$ que satisfaz

$$
L(v)=i c
$$

em $V_{0}$. Sem perda de generalidade podemos supor que $V_{0}$ é convexo. Considere

$$
\begin{aligned}
M: C^{\infty}\left(V_{0}\right) & \longrightarrow C^{\infty}\left(V_{0}\right) \\
f & \longmapsto e^{v} L(f)
\end{aligned}
$$

$M$ está bem definido, e além disso, $M$ é um campo em $V_{0}$ pelo que foi visto na seção 1.5 .

Observe que dado $u \in \mathscr{D}^{\prime}\left(V_{0}\right)$

$$
\left\langle{ }^{t} M(u), \varphi\right\rangle=\left\langle u, e^{v} L(\varphi)\right\rangle=\left\langle e^{v} u, L(\varphi)\right\rangle=\left\langle{ }^{t} L\left(e^{v} u\right), \varphi\right\rangle
$$


para toda $\varphi \in C_{c}^{\infty}\left(V_{0}\right)$. Ou seja, ${ }^{t} M={ }^{t} L\left(e^{v} \cdot\right)$. Por outro lado,

$$
\begin{aligned}
{ }^{t} M(u) & ={ }^{t} L\left(e^{v} u\right)=-L\left(e^{v} u\right)+i c e^{v} u \\
& =-L\left(e^{v}\right) u-e^{v} L(u)+i c e^{v} u=-e^{v} L(v) u-e^{v} L(u)+i c e^{v} u \\
& =-e^{v} i c u-e^{v} L(u)+i c e^{v} u \\
& =-e^{v} L(u)=-M(u) .
\end{aligned}
$$

E assim, segue do exemplo 1.3.7 que $\frac{\partial M\left(\pi_{1}\right)}{\partial x_{1}}+\frac{\partial M\left(\pi_{2}\right)}{\partial x_{2}}=0$, em $V_{0}$.

Logo, pelo teorema 1.5.18 a 1-forma complexa $\omega \doteq-M\left(\pi_{2}\right) d x_{1}+M\left(\pi_{1}\right) d x_{2}$ definida em $V_{0}$ é exata. Então, existe $u \in C^{\infty}\left(V_{0}\right)$ tal que $d u=\omega$ em $V_{0}$. Isto é,

$$
\frac{\partial u}{\partial x_{1}}=-M\left(\pi_{2}\right) \text { e } \frac{\partial u}{\partial x_{2}}=M\left(\pi_{1}\right)
$$

em $V_{0}$. Logo

$$
M(u)=M\left(\pi_{1}\right) \frac{\partial u}{\partial x_{1}}+M\left(\pi_{2}\right) \frac{\partial u}{\partial x_{2}}=-M\left(\pi_{1}\right) M\left(\pi_{2}\right)+M\left(\pi_{2}\right) M\left(\pi_{1}\right)=0,
$$

em $V_{0}$. Como $M=e^{v} L$, temos que $L(u)=0$ em $V_{0}$. Por fim, resta mostrar que $d u(x) \neq 0$ para todo $x \in V_{0}$. Basta notar que

$$
\left|-M\left(\pi_{2}\right)\right|+\left|M\left(\pi_{1}\right)\right|=\left|e^{v}\right|\left(\left|L\left(\pi_{1}\right)\right|+\left|L\left(\pi_{1}\right)\right|\right) \neq 0,
$$

em $V_{0}$, pois $L$ é não singular. Pela arbitrariedade de $x_{0} \in \Omega$, a proposição esta demonstrada.

Obs. 3.5.2. Em [24] podemos encontrar um exemplo de um campo vetorial complexo definido em um aberto de $\mathbb{R}^{2}$ que não é localmente integrável.

Corolário 3.5.3. Se L é um campo não singular e hipoelítico em $\Omega \subset \mathbb{R}^{2}$, então $L$ é localmente integrável. 
Demonstração: Aplicação direta do corolário 3.3.6 e da proposição 3.5.1.

Obs. 3.5.4. Na verdade, a classe dos $\mathrm{ODPL}$ que são localmente $C^{\infty}$ resolúveis é bem maior do que a classe dos operadores de tipo principal hipoeliticos. Em 1978 Hörmander [12] mostrou o seguinte resultado:

Seja $P(x, D)$ um $\boldsymbol{O D P L}$ de tipo principal definido $\Omega$. Se $P(x, D)$ satisfaz a condição $(P)$, então $P(x, D)$ é localmente $C^{\infty}$ resolúvel em $\Omega$.

Assim podemos concluir:

Se L é um campo não singular definido em $\Omega$ um aberto do $\mathbb{R}^{2}$ e $L(x, D)$ satisfaz a condição (P), então L é localmente integrável. 


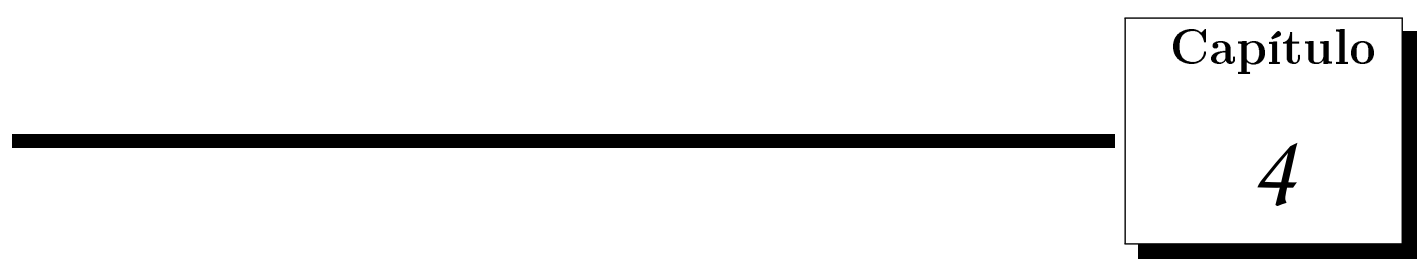

\section{Caso Elítico}

\subsection{Introdução}

Neste capítulo vamos mostrar que se $L$ é um campo vetorial complexo elítico definido em um aberto $\Omega \subset \mathbb{R}^{2}$ com $\pi_{1}(\Omega)=\{0\}$ ou $\pi_{1}(\Omega)=\mathbb{Z}$, então o problema

$\left(*^{\prime}\right) \quad L Z=0, \operatorname{grad}(\Re\{Z\}), \operatorname{grad}(\Im\{Z\})$ linearmente independentes e $Z$ injetiva em $\Omega$ tem solução global. Consequentemente vamos obter uma mudança global de variáveis que transforma $L$ em um múltiplo do operador de Cauchy - Riemann.

\subsection{Eliticidade e Integrabilidade Local}

Nesta seção vamos caracterizar campos vetoriais complexos elíticos definidos em abertos de $\mathbb{R}^{2}$ em função da integrabilidade local.

Teorema 4.2.1. Seja L um campo vetorial complexo não singular definido em um aberto conexo $\Omega$ do $\mathbb{R}^{2}$. Então as seguintes afirmações são equivalentes:

1. L é elítico em $\Omega$.

2. existe uma familia $\left\{\left(U_{\alpha}, Z_{\alpha}\right)\right\}_{\alpha \in I}$ tal que $\left\{U_{\alpha}\right\}_{\alpha \in I}$ é uma cobertura aberta de $\Omega$ e para cada $\alpha \in I$, existe uma função $C^{\infty}$ injetiva $Z_{\alpha}: U_{\alpha} \longrightarrow \mathbb{C}$, tal que $L Z_{\alpha}=0$, $\operatorname{grad}\left(\Re\left\{Z_{\alpha}\right\}\right), \operatorname{grad}\left(\Im\left\{Z_{\alpha}\right\}\right)$ são linearmente independentes em $U_{\alpha}$. 
Demonstração: Vamos começar demonstrando que (2 $\Longrightarrow 1$ ).

Seja $p_{0} \in \Omega$. Por hipótese, existe $\alpha \in I$ tal que $p_{0} \in U_{\alpha}$ e $L Z_{\alpha}=0$ em $U_{\alpha}$. Pela proposição 1.5.19 a menos de um múltiplo $C^{\infty}$ que não se anula, podemos escrever

$$
L=\partial_{x} Z_{\alpha} \frac{\partial}{\partial t}-\partial_{t} Z_{\alpha} \frac{\partial}{\partial x} \text { em } U_{\alpha}
$$

Note que $\partial_{x} Z_{\alpha} \neq 0$ em $U_{\alpha}$. De fato, $\partial_{x} Z_{\alpha}=0$ em $p \in U_{\alpha}$ se, e somente se, $\partial_{x} \Re\left\{Z_{\alpha}\right\}=0$ e $\partial_{x} \Im\left\{Z_{\alpha}\right\}=0$ em $p$, o que implica $\operatorname{grad}\left(\Re\left\{Z_{\alpha}\right\}\right)$ e $\operatorname{grad}\left(\Im\left\{Z_{\alpha}\right\}\right)$ linearmente dependentes em $p$, o que contradiz 2. Assim, em $U_{\alpha}$ podemos escrever

$$
\begin{aligned}
L & =\partial_{x} Z_{\alpha}\left(\frac{\partial}{\partial t}-\frac{\partial_{t} Z_{\alpha}}{\partial_{x} Z_{\alpha}} \frac{\partial}{\partial x}\right) \\
& =\partial_{x} Z_{\alpha}\left(\frac{\partial}{\partial t}-\frac{\overline{\partial_{x} Z_{\alpha}} \partial_{t} Z_{\alpha}}{\left|\partial_{x} Z_{\alpha}\right|^{2}} \frac{\partial}{\partial x}\right) \\
& =\partial_{x} Z_{\alpha}\left(\frac{\partial}{\partial t}-\Re\left\{\frac{\overline{\partial_{x} Z_{\alpha}} \partial_{t} Z_{\alpha}}{\left|\partial_{x} Z_{\alpha}\right|^{2}}\right\} \frac{\partial}{\partial x}\right)+i \partial_{x} Z_{\alpha} \Im\left\{\frac{\overline{\partial_{x} Z_{\alpha}} \partial_{t} Z_{\alpha}}{\left|\partial_{x} Z_{\alpha}\right|^{2}}\right\} \frac{\partial}{\partial x}
\end{aligned}
$$

Assim, é fácil ver que $L$ é elítico em $U_{\alpha}$ se, e somente se, $\Im\left\{\frac{\overline{\partial_{x} Z_{\alpha}} \partial_{t} Z_{\alpha}}{\left|\partial_{x} Z_{\alpha}\right|^{2}}\right\}(x, t) \neq 0$, para todo $(x, t) \in U_{\alpha}$. Note que

$$
\begin{gathered}
\Im\left\{\frac{\overline{\partial_{x} Z_{\alpha}} \partial_{t} Z_{\alpha}}{\left|\partial_{x} Z_{\alpha}\right|^{2}}\right\}=\frac{\partial_{x} \Re\left\{Z_{\alpha}\right\} \partial_{t} \Im\left\{Z_{\alpha}\right\}-\partial_{x} \Im\left\{Z_{\alpha}\right\} \partial_{t} \Re\left\{Z_{\alpha}\right\}}{\left|\partial_{x} Z_{\alpha}\right|^{2}} \\
=\frac{1}{\left|\partial_{x} Z_{\alpha}\right|^{2}}\left|\begin{array}{ll}
\frac{\partial \Re\left\{Z_{\alpha}\right\}}{\partial x} & \frac{\partial \Re\left\{Z_{\alpha}\right\}}{\partial t} \\
\frac{\partial \Im\left\{Z_{\alpha}\right\}}{\partial x} & \frac{\partial \Im\left\{Z_{\alpha}\right\}}{\partial t}
\end{array}\right| \neq 0
\end{gathered}
$$

em $U_{\alpha}$, pela independência linear dos vetores $\operatorname{grad}\left(\Re\left\{Z_{\alpha}\right\}\right), \operatorname{grad}\left(\Im\left\{Z_{\alpha}\right\}\right)$ em $U_{\alpha}$. Portanto, $L$ é elítico em $U_{\alpha}$ e em particular em $p_{0}$. Como $p_{0}$ é um ponto arbitrário de $\Omega, L$ é elítico em $\Omega$.

$(1 \Longrightarrow 2)$ Como vimos no teorema 1.5.8, podemos escrever

$$
L=L\left(\pi_{1}\right) \frac{\partial}{\partial x}+L\left(\pi_{2}\right) \frac{\partial}{\partial t}
$$


em $\Omega$, onde $\pi_{1}, \pi_{2}$ são as projeções canônicas do $\mathbb{R}^{2}$ em relação à primeira e à segunda coordenadas respectivamente. Como $L$ é elítico, $L\left(\pi_{1}\right)(x, t) \neq 0$ e $L\left(\pi_{2}\right)(x, y) \neq 0$ para todo $(x, t) \in \Omega$. Assim podemos escrever

$$
L=L\left(\pi_{2}\right)\left[\frac{\partial}{\partial t}+\frac{L\left(\pi_{1}\right)}{L\left(\pi_{2}\right)} \frac{\partial}{\partial x}\right]
$$

em $\Omega$. Como estamos procurando soluções locais da homogênea, com boas propriedades, não há perda de generalidade em supor

$$
L=\frac{\partial}{\partial t}+\frac{L\left(\pi_{1}\right)}{L\left(\pi_{2}\right)} \frac{\partial}{\partial x} \text { em } \Omega
$$

Sejam $f \doteq \Re\left\{\frac{L\left(\pi_{1}\right)}{L\left(\pi_{2}\right)}\right\}$ e $g \doteq \Im\left\{\frac{L\left(\pi_{1}\right)}{L\left(\pi_{2}\right)}\right\}$. Então podemos escrever

$$
L=\frac{\partial}{\partial t}+(f+i g) \frac{\partial}{\partial x} \text { em } \Omega
$$

Temos que $f$ e $g$ são funções reais, e como $L$ é elítico em $\Omega$ temos que $g$ não se anula em $\Omega$. De fato, temos que $L$ é elítico em $\Omega$ se, e somente se, o operador diferencial $L(p, D)=i D_{t}+i(f(p)+i g(p)) D_{x}$ é elítico em $\Omega$. Mas $L(p, D)$ é elítico em $\Omega$ se, e somente se, para todo $p \in \Omega, L(p, \xi) \neq 0$ para todo $\xi \in \mathbb{R}^{2} \backslash\{0\}$. Suponha por contradição que existe $p_{0} \in \Omega$ tal que $g\left(p_{0}\right)=0$. Então $L\left(p_{0}, \xi\right)=i \xi_{2}+i f\left(p_{0}\right) \xi_{1}=i\left(\xi_{2}+f\left(p_{0}\right) \xi_{1}\right)$ para todo $\xi \in \mathbb{R}^{2} \backslash\{0\}$. Logo tomando $\xi_{0}=\left(1,-f\left(p_{0}\right)\right)$, temos $L\left(p_{0}, \xi_{0}\right)=0 \operatorname{com} \xi_{0} \in \mathbb{R}^{2} \backslash\{0\}$. Assim, $L$ não é elítico em $p_{0}$. Contradição. Portanto $g$ não se anula em $\Omega$.

Observe que pelo fato de $g$ não se anular em $\Omega$ e de $\Omega$ ser um aberto conexo, temos que $g$ não muda de sinal em $\Omega$. A menos de uma mudança de variáveis, podemos supor que $g(x, t)<0$ para todo $(x, t) \in \Omega$. De fato, se $g(x, t)>0$ para todo $(x, t) \in \Omega$, considere o difeomorfimo

$$
F(x, t)=(-x, t),(x, t) \in \Omega
$$

Então,

$$
L_{F}=\frac{\partial}{y_{2}}+(\tilde{f}+i \tilde{g}) \frac{\partial}{\partial y_{1}}
$$


em $F(\Omega)=\left\{\left(y_{1}, y_{2}\right) \in \mathbb{R}^{2} ;\left(-y_{1}, y_{2}\right) \in \Omega\right\}$, onde $\tilde{f}\left(y_{1}, y_{2}\right)=-f\left(-y_{1}, y_{2}\right)$ e $\tilde{g}\left(y_{1}, y_{2}\right)=$ $-g\left(-y_{1}, y_{2}\right),\left(y_{1}, y_{2}\right) \in F(\Omega), \log \tilde{g}<0$.

Seja $p_{0} \in \Omega$. Suponha que $Z$ é solução local da homogênea em uma vizinhança de $p_{0}$, i.e., $L Z=0$ em $U$, onde $U$ é um aberto de $\Omega$ que contém $p_{0}$.

Obs. 4.2.2. Observe que $L Z=0$ em $U$, implica $Z \in C^{\infty}(U)$, uma vez que $L$ é um campo hipoelítico.

Temos que $L Z=0$ em $U$

$$
\Longleftrightarrow\left(\frac{\partial \Re\{Z\}}{\partial t}+f \frac{\partial \Re\{Z\}}{\partial x}-g \frac{\partial \Im\{Z\}}{\partial x}\right)+i\left(\frac{\partial \Im\{Z\}}{\partial t}+f \frac{\partial \Im\{Z\}}{\partial x}+g \frac{\partial \Re\{Z\}}{\partial x}\right)=0
$$

em $U$

$$
\Longleftrightarrow \frac{\partial \Re\{Z\}}{\partial t}+f \frac{\partial \Re\{Z\}}{\partial x}-g \frac{\partial \Im\{Z\}}{\partial x}=0 \text { e } \frac{\partial \Im\{Z\}}{\partial t}+f \frac{\partial \Im\{Z\}}{\partial x}+g \frac{\partial \Re\{Z\}}{\partial x}=0
$$

em $U$.

Assim, encontrar uma função $Z$ que satisfaça $L Z=0$ em $U, \operatorname{com} \operatorname{grad}(\Re\{Z\})$, $\operatorname{grad}(\Im\{Z\})$ linearmente independentes em $U$, é equivalente a encontrar um par de funções $u(x, t), v(x, t)$ definidas em $U$, a valores reais, que satisfaz o seguinte sistema de equações:

$$
\left\{\begin{array}{l}
u_{t}=-a v_{x}-b v_{t} \\
u_{x}=b v_{x}+c v_{t}
\end{array}\right.
$$

com a condição $u_{x} v_{t}-u_{t} v_{x} \neq 0$, onde

$$
a=-\left(\frac{f^{2}}{g}+g\right), \quad b=-\frac{f}{g}, \quad c=-\frac{1}{g} .
$$

Obs. 4.2.3. As funções a,b e c estão em $C^{\infty}(\Omega)$. De fato, basta notar que $f, g \in C^{\infty}(\Omega)$ e $g \neq 0$ em $\Omega$.

Considere

$$
\mu \doteq \frac{c-a-2 i b}{c+a+2}
$$

em $\Omega$. A função $\mu$ é chamada de coeficiente de Beltrami do campo L. 
Af. 4.2.4. $\mu$ está bem definida e $\mu: \Omega \longrightarrow \mathbb{C}$ é $C^{\infty}$.

Como $a, b$ e $c \in C^{\infty}(\Omega)$ basta verificar que $(c+a+2)(p) \neq 0$, para todo $p \in \Omega$. Suponha por contradição a existência de $q \in \Omega$, tal que $(c+a+2)(q)=0$. Então

$$
\begin{gathered}
-\frac{1}{g(q)}-\left(\frac{f^{2}}{g}+g\right)(q)+2=0 \\
\Longrightarrow \quad 1-2 g(q)+g^{2}(q)+f^{2}(q)=0 \\
\Longrightarrow \quad(g-1)^{2}(q)+f^{2}(q)=0
\end{gathered}
$$

Portanto $g(q)=1$ e $f(q)=0$. Contradição, pois $g<0$ em $\Omega$.

Af. 4.2.5. $|\mu|<1$ em $\Omega$.

Com alguns cálculos temos

$$
|\mu|^{2}=\frac{|c-a-2 i b|^{2}}{|c+a+2|^{2}}=1-\frac{4}{c+a+2} \text { em } \Omega .
$$

Logo, $|\mu|^{2}<1$ em $\Omega$ se, e somente se $c+a+2>0$ em $\Omega$.

Na argumentação da afirmação 4.2.4 vimos que $c+a+2 \neq 0$ em $\Omega$. Como $g<0$ em $\Omega$, olhando para a igualdade abaixo

$$
c+a+2=-\frac{1}{g}-\left(\frac{f^{2}}{g}+g\right)+2
$$

é fácil ver que $c+a+2>0$ em $\Omega$. Portanto $|\mu|^{2}<1$ em $\Omega$ e daí, $|\mu|<1$ em $\Omega$.

Lema 4.2.6. Encontrar um par de funções $u, v$ a valores reais que satisfaz o sistema de equações

$$
\left\{\begin{array}{l}
u_{t}=-a v_{x}-b v_{t} \\
u_{x}=b v_{x}+c v_{t}
\end{array}\right.
$$

onde $a, b$ e c são as funções reais em (4.2.1), satisfazendo a condição $u_{x} v_{t}-u_{t} v_{x} \neq 0$ é equivalente a encontrar uma solução $Z=u+i v$ da equação equações de Beltrami

$$
\frac{\partial Z}{\partial \bar{z}}=\mu \frac{\partial Z}{\partial z}, \quad \mu=\frac{c-a-2 i b}{c+a+2}
$$

satisfazendo a condição $\frac{\partial Z}{\partial z} \neq 0$. 
Note que qualquer solução de (4.2.4) é $C^{\infty}$ pela observação A.2.5.

Demonstração do lema: Diretamente de (4.2.3) obtemos

$$
\begin{aligned}
& 2 \frac{\partial Z}{\partial \bar{z}}=(b-i a+i) v_{x}+(c-i b-1) v_{t} \\
& 2 \frac{\partial Z}{\partial z}=(b+i a+i) v_{x}+(c+i b+1) v_{t} .
\end{aligned}
$$

Com um simples cálculo mostramos que os coeficientes de $v_{x}$ e $v_{t}$ são proporcionais, basta notar que

$$
\left|\begin{array}{cc}
(b-i a+i) & (c-i b-1) \\
(b+i a+i) & (c+i b+1)
\end{array}\right|=0 .
$$

Mais ainda, os coeficientes $(b+i a+i)$ e $(c+i b+1)$ são não nulos em $\Omega$.

Com essas observações, de (4.2.5) obtemos

$$
\begin{aligned}
& 2 \frac{\partial Z}{\partial \bar{z}}=(b-i a+i) v_{x}+\left(\frac{c+i b+1}{b+i a+i}\right)(b-i a+i) v_{t}, \\
& 2 \frac{\partial Z}{\partial z}=(b+i a+i) v_{x}+\left(\frac{c+i b+1}{b+i a+i}\right)(b+i a+i) v_{y} t .
\end{aligned}
$$

Ou seja,

$$
\begin{aligned}
\frac{\partial Z}{\partial \bar{z}} & =\frac{1}{2}(b-i a+i)\left(v_{x}+\left(\frac{c+i b+1}{b+i a+i}\right) v_{t}\right), \\
\frac{\partial Z}{\partial z} & =\frac{1}{2}(b+i a+i)\left(v_{x}+\left(\frac{c+i b+1}{b+i a+i}\right) v_{t}\right) .
\end{aligned}
$$

Daí temos

$$
\frac{\partial \omega}{\partial \bar{z}}=\left(\frac{b-a i+i}{b+i a+i}\right) \frac{\partial \omega}{\partial z}=\left(\frac{c-a-2 i b}{c+a+2}\right) \frac{\partial \omega}{\partial z}=\mu \frac{\partial \omega}{\partial z} .
$$

Segue daí que (4.2.3) e (4.2.4) são equivalentes.

Note que $\frac{c+i b+1}{b+i a+i}=\frac{b-i}{a}$ em $\Omega$ e $\Im\left\{\frac{b-i}{a}\right\} \neq 0$ em $\Omega$. Assim, pelo fato de $(b+i a+i) \neq 0$ em $\Omega$ e por (4.2.6) obtemos: $\frac{\partial Z}{\partial z}=0$ se, e somente se $v_{x}=v_{t}=0$.

Observe que

$$
u_{x} v_{t}-u_{t} v_{x}=\left(b v_{x}+c v_{t}\right) v_{t}+\left(a v_{x}+b v_{t}\right) v_{x}=a v_{x}^{2}+2 b v_{x} v_{t}+c v_{t}^{2}
$$




$$
=\left(\frac{-1}{g}\right)\left[\left(f v_{x}+v_{t}\right)^{2}+g^{2} v_{x}^{2}\right]
$$

Logo $u_{x} v_{t}-u_{t} v_{x}=0$ se, e somente se $v_{x}=v_{t}=0$. Portanto $u_{x} v_{t}-u_{t} v_{x} \neq 0$ se, e somente se $\frac{\partial Z}{\partial z} \neq 0$. Assim, o lema está demonstrado.

Portanto, para concluir a demonstração do teorema 4.2.1, basta obter, para cada ponto $p_{0} \in \Omega$, uma função $Z: U \longrightarrow \mathbb{C}$, onde $U$ é uma vizinhança aberta de $p_{0}$ em $\Omega$, de tal modo que $Z$ satisfaça a equação de Beltrami dada em (4.2.4). Para resolver tal problema, vamos usar o seguinte resultado, cuja demonstração se encontra no apêndice A (mais precisamente, corolário A.2.6).

Seja $\mu \in C^{\infty}(\Omega)$, onde $\Omega$ é um aberto de $\mathbb{R}^{2}$, com $|\mu|<1$ em $\Omega$. Então para cada $p_{0} \in \Omega$, existe uma função $C^{\infty} Z: U \longrightarrow \mathbb{C}$, onde $U$ é uma vizinhança aberta de $p_{0}$ em $\Omega$, tal que

$$
\frac{\partial Z}{\partial \bar{z}}=\mu \frac{\partial Z}{\partial z} \quad e \quad \frac{\partial Z}{\partial z} \neq 0 \text { em } U
$$

E assim, concluímos a demonstração de teorema 4.2.1.

Exemplo 4.2.7. Seja

$$
L=\frac{\partial}{\partial t}-i t \frac{\partial}{\partial x}
$$

definido em $H^{+}=\left\{(x, t) \in \mathbb{R}^{2} ; t>0\right\}$. Temos que L é elítico em $H^{+}$e o coeficiente de Beltrami de L é

$$
\mu(x, t)=\frac{1-t}{1+t},(x, t) \in H^{+}
$$

Note que $\mu(x, t) \longrightarrow 1$ quando $(x, t)$ tende a fronteira de $H^{+}$. 


\subsubsection{Redução de um Campo Elítico a um múltiplo do Operador de Cauchy-Riemann}

Uma consequência do teorema 4.2.1 é um resultado clássico dado pelo corolário 4.2.8, que diz que localmente, a menos de uma mudança de coordenadas, um campo elítico planar é um múltiplo do operador de Cauchy-Riemann. Podemos pensar, que em certo sentido, os campos elíticos podem ser diagonalizados.

Corolário 4.2.8. Se L é um campo vetorial complexo elítico definido no aberto conexo $\Omega$ do $\mathbb{R}^{2}$, então existe uma cobertura aberta $\left\{U_{\alpha}\right\}_{\alpha \in I}$ de $\Omega$ tal que para cada $\alpha \in I$ existe um difeomorfismo $F_{\alpha}: U_{\alpha} \longrightarrow F_{\alpha}\left(U_{\alpha}\right)$ de classe $C^{\infty}$ tal que $L_{F_{\alpha}}$ é um múltiplo do operador de Cauchy-Riemann em $F_{\alpha}\left(U_{\alpha}\right)$, Mais precisamente,

$$
L_{F_{\alpha}}=\lambda_{\alpha} \frac{\partial}{\partial \bar{w}}, w=y_{1}+i y_{2},
$$

onde $\lambda_{\alpha} \in C^{\infty}\left(F_{\alpha}\left(U_{\alpha}\right)\right)$ é tal que $\lambda\left(y_{1}, y_{2}\right) \neq 0, \forall\left(y_{1}, y_{2}\right) \in F_{\alpha}\left(U_{\alpha}\right)$.

\section{Demonstração:}

Pelo teorema 4.2.1, existe uma cobertura aberta $\left\{U_{\alpha}\right\}_{\alpha \in I}$ de $\Omega$, e para cada $\alpha \in I$ existe uma função $Z_{\alpha} \longrightarrow \mathbb{C}$ tal que $L Z_{\alpha}=0, Z_{\alpha}: U_{\alpha} \longrightarrow \mathbb{C}$ injetiva e $\operatorname{grad}\left(\Re\left\{Z_{\alpha}\right\}\right)$, $\operatorname{grad}\left(\Im\left\{Z_{\alpha}\right\}\right)$ são linearmente independentes em $U_{\alpha}$. Assim para cada $\alpha \in I$, definimos

$$
F_{\alpha}=\left(\Re\left\{Z_{\alpha}\right\}, \Im\left\{Z_{\alpha}\right\}\right) \text { em } U_{\alpha}
$$

Como $\operatorname{grad}\left(\Re\left\{Z_{\alpha}\right\}\right), \operatorname{grad}\left(\Im\left\{Z_{\alpha}\right\}\right)$ são linearmente independentes em $U_{\alpha}$, obtemos que $\operatorname{det}\left(J\left(F_{\alpha}\right)\right) \neq 0$ em $U_{\alpha}$. Daí, temos que $F_{\alpha}$ é um difeomorfismo $C^{\infty}$ do aberto $U_{\alpha}$ sobre o aberto $F_{\alpha}\left(U_{\alpha}\right)$.

Obs. 4.2.9. Vamos considerar um elemento genérico de $F_{\alpha}\left(U_{\alpha}\right)$ como sendo $\left(y_{1}, y_{2}\right)$.

Escrevendo $L$ nas novas coordenadas induzidas por $F_{\alpha}$, temos

$$
\begin{gathered}
L_{F_{\alpha}}=\left(L\left(F_{\alpha 1}\right) \circ F_{\alpha}^{-1}\right) \frac{\partial}{\partial y_{1}}+\left(L\left(F_{\alpha 2}\right) \circ F_{\alpha}^{-1}\right) \frac{\partial}{\partial y_{2}} \\
=\left(L\left(F_{\alpha 1}\right) \circ F_{\alpha}^{-1}\right) \frac{\partial}{\partial y_{1}}+\left(L\left(F_{\alpha 2}\right) \circ F_{\alpha}^{-1}\right) \frac{\partial}{\partial y_{2}}+i\left(L\left(F_{\alpha 2}\right) \circ F_{\alpha}^{-1}\right) \frac{\partial}{\partial y_{1}}-i\left(L\left(F_{\alpha 2}\right) \circ F_{\alpha}^{-1}\right) \frac{\partial}{\partial y_{1}}
\end{gathered}
$$




$$
\begin{gathered}
=\left(L\left(F_{\alpha 1}+i F_{\alpha 2}\right) \circ F_{\alpha}^{-1}\right) \frac{\partial}{\partial y_{1}}+\left(L\left(F_{\alpha 2}\right) \circ F_{\alpha}^{-1}\right)\left(\frac{\partial}{\partial y_{2}}-i \frac{\partial}{\partial y_{1}}\right) \\
=\left(L\left(Z_{\alpha}\right) \circ F_{\alpha}^{-1}\right) \frac{\partial}{\partial y_{1}}-i\left(L\left(F_{\alpha 2}\right) \circ F_{\alpha}^{-1}\right)\left(\frac{\partial}{\partial y_{1}}+i \frac{\partial}{\partial y_{2}}\right) \\
=-i\left(L\left(F_{\alpha 2}\right) \circ F_{\alpha}^{-1}\right)\left(\frac{\partial}{\partial y_{1}}+i \frac{\partial}{\partial y_{2}}\right)
\end{gathered}
$$

Analogamente,

$$
\begin{gathered}
L_{F_{\alpha}}=\left(L\left(F_{\alpha 1}\right) \circ F_{\alpha}^{-1}\right) \frac{\partial}{\partial y_{1}}+\left(L\left(F_{\alpha 2}\right) \circ F_{\alpha}^{-1}\right) \frac{\partial}{\partial y_{2}} \\
=\left(L\left(F_{\alpha 1}\right) \circ F_{\alpha}^{-1}\right) \frac{\partial}{\partial y_{1}}+\left(L\left(F_{\alpha 2}\right) \circ F_{\alpha}^{-1}\right) \frac{\partial}{\partial y_{2}}-i\left(L\left(F_{\alpha 1}\right) \circ F_{\alpha}^{-1}\right) \frac{\partial}{\partial y_{2}}+i\left(L\left(F_{\alpha 1}\right) \circ F_{\alpha}^{-1}\right) \frac{\partial}{\partial y_{2}} \\
=-i\left(L\left(F_{\alpha 1}+i F_{\alpha 2}\right) \circ F_{\alpha}^{-1}\right) \frac{\partial}{\partial y_{2}}+\left(L\left(F_{\alpha 1}\right) \circ F_{\alpha}^{-1}\right)\left(\frac{\partial}{\partial y_{2}}+i \frac{\partial}{\partial y_{1}}\right) \\
=-i\left(L\left(Z_{\alpha}\right) \circ F_{\alpha}^{-1}\right) \frac{\partial}{\partial y_{1}}+\left(L\left(F_{\alpha 1}\right) \circ F_{\alpha}^{-1}\right)\left(\frac{\partial}{\partial y_{1}}+i \frac{\partial}{\partial y_{2}}\right) \\
=\left(L\left(F_{\alpha 1}\right) \circ F_{\alpha}^{-1}\right)\left(\frac{\partial}{\partial y_{1}}+i \frac{\partial}{\partial y_{2}}\right) .
\end{gathered}
$$

Portanto,

$$
2 L_{F_{\alpha}}=\left(L\left(F_{\alpha 1}-i F_{\alpha 2}\right) \circ F_{\alpha}^{-1}\right)\left(\frac{\partial}{\partial y_{1}}+i \frac{\partial}{\partial y_{2}}\right)=\left(L\left(\overline{Z_{\alpha}}\right) \circ F_{\alpha}^{-1}\right)\left(\frac{\partial}{\partial y_{1}}+i \frac{\partial}{\partial y_{2}}\right)
$$

Ou seja,

$$
L_{F_{\alpha}}=\left(L\left(\overline{Z_{\alpha}}\right) \circ F_{\alpha}^{-1}\right) \frac{1}{2}\left(\frac{\partial}{\partial y_{1}}+i \frac{\partial}{\partial y_{2}}\right)=\lambda_{\alpha} \frac{\partial}{\partial \bar{w}}
$$

onde $w=y_{1}+i y_{2}$ e $\lambda_{\alpha} \doteq L\left(\overline{Z_{\alpha}}\right) \circ F_{\alpha}^{-1}$ em $F_{\alpha}\left(U_{\alpha}\right)$. Como a eliticidade é invariante por mudança de coordenadas, segue que $\lambda_{\alpha}\left(y_{1}, y_{2}\right) \neq 0$, para todo $\left(y_{1}, y_{2}\right) \in F_{\alpha}\left(U_{\alpha}\right)$.

Exemplo 4.2.10. Considere

$$
L=\frac{\partial}{\partial t}-i t \frac{\partial}{\partial x}
$$

definido em $H^{+}=\left\{(x, t) \in \mathbb{R}^{2} \mid t>0\right\}$. Temos que L é elítico em $H^{+}$e com um simples cálculo, obtemos que $Z(x, t)=x+i \frac{t^{2}}{2},(x, t) \in H^{+}$é solução, isto é, $L Z=0$ em $H^{+}$. Associado a $Z$, considere o difeomorfismo 


$$
F(x, t)=\left(x, t^{2} / 2\right),(x, t) \in H^{+} .
$$

Note que $F\left(H^{+}\right)=H^{+}$. Temos

$$
L_{F}=-2 i \sqrt{2 y_{2}} \frac{\partial}{\partial \bar{w}}
$$

$\lambda\left(y_{1}, y_{2}\right)=-2 i \sqrt{2 y_{2}},\left(y_{1}, y_{2}\right) \in H^{+}$, onde $w=y_{1}+i y_{2}$. Observe que $\lambda(x, t) \longrightarrow 0$, quando $(x, t) \longrightarrow\left(x_{0}, 0\right)$, para qualquer $x_{0} \in \mathbb{R}$.

Surge de forma natural, em relação ao corolário 4.2.8, o corolário 4.2.11 abaixo, que trata da representação das soluções de um campo elítico. No capítulo 5 discutiremos uma versão deste resultado para campos hipoelíticos.

Corolário 4.2.11. Seja $L$ um campo vetorial complexo elítico definido no aberto $\Omega$ de $\mathbb{R}^{2}$. Associada a L considere a família $\left\{\left(U_{\alpha}, Z_{\alpha}\right)\right\}_{\alpha \in I}$ dada pelo teorema 4.2.1. Seja $p_{0} \in \Omega$. Se $u \in \mathscr{D}^{\prime}(V), V \subset \Omega$ vizinhança aberta de $p_{0}$, é tal que Lu $=0$ em $V$, então para cada $\alpha \in I$ com $p_{0} \in V \cap U_{\alpha}$ existe uma função holomorfa $h: Z_{\alpha}\left(V \cap U_{\alpha}\right) \longrightarrow \mathbb{C}$ tal que

$$
u=h \circ Z_{\alpha} e m V \cap U_{\alpha}
$$

\section{Demonstração:}

Inicialmente, observe que $u \in C^{\infty}(V)$, pois, $L u=0$ em $V$ e $L$ é hipoelítico em $V$. Seja $\alpha \in I$ tal que $p_{0} \in U_{\alpha}$. Assim $p_{0} \in V \cap U_{\alpha}$. Como foi feito na demonstração do corolário 4.2.8, definimos

$$
F_{\alpha}=\left(\Re\left\{Z_{\alpha}\right\}, \Im\left\{Z_{\alpha}\right\}\right) \text { em } U_{\alpha}
$$

Temos que

$$
L_{F_{\alpha}}=\lambda_{\alpha} \frac{\partial}{\partial \bar{w}}, w=y_{1}+i y_{2},
$$

onde $\lambda_{\alpha} \in C^{\infty}\left(F_{\alpha}\left(U_{\alpha}\right)\right)$ é tal que $\lambda\left(y_{1}, y_{2}\right) \neq 0, \forall\left(y_{1}, y_{2}\right) \in F_{\alpha}\left(U_{\alpha}\right)$. Pela proposição 1.5.24 temos que $F_{\alpha}^{*}(u)=u \circ F_{\alpha}^{-1}$ é solução de $L_{F_{\alpha}}$ em $F_{\alpha}\left(V \cap U_{\alpha}\right)$, isto é, $L_{F_{\alpha}} F_{\alpha}^{*}(u)=0 \mathrm{em}$ $F_{\alpha}\left(V \cap U_{\alpha}\right)$. Como $\lambda\left(y_{1}, y_{2}\right) \neq 0, \forall\left(y_{1}, y_{2}\right) \in F_{\alpha}\left(U_{\alpha}\right)$, temos

$$
\frac{\partial}{\partial \bar{w}} F_{\alpha}^{*}(u)=0 \text { em } F_{\alpha}\left(V \cap U_{\alpha}\right)
$$


Logo $F_{\alpha}^{*}(u)$ na variável $w=y_{1}+i y_{2}$, é uma função holomofa, isto é,

$$
h(w) \doteq F_{\alpha}^{*}(u)\left(y_{1}, y_{2}\right),
$$

é uma função holomofa no aberto $Z_{\alpha}\left(V \cap U_{\alpha}\right) \subset \mathbb{C}$, lembrando que

$$
Z_{\alpha}\left(V \cap U_{\alpha}\right)=\left\{y_{1}+i y_{2} ;\left(y_{1}, y_{2}\right) \in F_{\alpha}\left(V \cap U_{\alpha}\right)\right\}
$$

Por fim, observe que

$$
u=\left(F_{\alpha}^{-1}\right)^{*}\left(F_{\alpha}^{*}(u)\right)=\left(u \circ F_{\alpha}^{-1}\right) \circ F_{\alpha}=h \circ Z_{\alpha} \text { em } V \cap U_{\alpha},
$$

lembrando que $Z_{\alpha}=F_{\alpha 1}+i F_{\alpha 2}$ em $U_{\alpha}$.

\subsection{Estrutura Diferenciável Complexa induzida por um Campo Elítico}

Seja $\Omega$ um aberto conexo de $\mathbb{R}^{2}$ e $L$ um campo vetorial complexo elítico definido em $\Omega$. Vamos considerar $\Omega$ como um subespaço topológico do $\mathbb{R}^{2}$. Assim, $\Omega$ é um espaço de Hausdorff conexo e com base enumerável.

Seja $\mathcal{A}=\left\{\left(U_{\alpha}, Z_{\alpha}\right)\right\}_{\alpha \in I}$ dada pelo teorema 4.2.1. Na demonstração do corolário 4.2.8 vimos que para cada $\alpha \in I$,

$$
F_{\alpha}=\left(\Re\left\{Z_{\alpha}\right\}, \Im\left\{Z_{\alpha}\right\}\right) \text { em } U_{\alpha}
$$

é um difeomorfismo do aberto $U_{\alpha}$ sobre o aberto $F_{\alpha}\left(U_{\alpha}\right)$. Como a aplicação $\mathbb{R}^{2} \ni(x, t) \mapsto$ $x+i t \in \mathbb{C}$ é um homeomorfismo, segue que a função $Z_{\alpha}: U_{\alpha} \longrightarrow Z_{\alpha}\left(U_{\alpha}\right)$ é um homeomorfismo. Além disso, $\left\{U_{\alpha}\right\}_{\alpha \in I}$ é uma cobertura de $\Omega$.

Agora, sejam $\alpha, \beta \in I$, tais que $U_{\alpha} \cap U_{\beta} \neq \emptyset$. Pelo corolário 4.2.11, existe uma função $h_{\alpha \beta}$ holomorfa em $Z_{\alpha}\left(U_{\alpha} \cap U_{\beta}\right)$ tal que

$$
Z_{\beta}=h_{\alpha \beta} \circ Z_{\alpha} \text { em } U_{\alpha} \cap U_{\beta}
$$


Daí,

$$
h_{\alpha \beta}=Z_{\beta} \circ Z_{\alpha}^{-1}: Z_{\alpha}\left(U_{\alpha} \cap U_{\beta}\right) \longrightarrow Z_{\beta}\left(U_{\alpha} \cap U_{\beta}\right)
$$

é uma função holomorfa injetora e sobrejetora, com inversa dada por

$$
h_{\beta \alpha}=Z_{\alpha} \circ Z_{\beta}^{-1}: Z_{\beta}\left(U_{\alpha} \cap U_{\beta}\right) \longrightarrow Z_{\alpha}\left(U_{\alpha} \cap U_{\beta}\right) .
$$

Ou seja, $h_{\alpha \beta}$ é um biholomorfismo entre os abertos $Z_{\beta}\left(U_{\alpha} \cap U_{\beta}\right)$ e $Z_{\alpha}\left(U_{\alpha} \cap U_{\beta}\right)$. Portanto, $\mathcal{A}$ é um atlas em $\Omega$.

Seja $\mathscr{A}=\left\{\left(U_{\alpha}, Z_{\alpha}\right)\right\}_{\alpha \in \mathcal{I}}$ a estrutura diferenciável complexa induzida pelo atlas $\mathcal{A} \mathrm{em}$ $\Omega$. A estrutura $\mathscr{A}$ também possui as boas propriedades do atlas $\mathcal{A}$ :

$L Z_{\alpha}=0$ em $U_{\alpha}$ e $\operatorname{grad}\left(\Re\left\{Z_{\alpha}\right\}\right), \operatorname{grad}\left(\Im\left\{Z_{\alpha}\right\}\right)$ são linearmente independentes em $U_{\alpha}$ para todo $\alpha \in \mathcal{I}$.

De fato, seja $\beta \in \mathcal{I}$ e seja $p_{0} \in U_{\beta}$. Como $\left\{U_{\alpha}\right\}_{\alpha \in I}$ é uma cobertura aberta de $\Omega$ existe $\alpha \in I$ tal que $p_{0} \in U_{\alpha_{0}}$. Como $\left(U_{\alpha}, Z_{\alpha_{0}}\right),\left(U_{\beta}, Z_{\beta}\right) \in \mathscr{A}$, existe um biholomorfismo $h$ de $Z_{\alpha}\left(U_{\alpha} \cap U_{\beta}\right)$ sobre $Z_{\beta}\left(U_{\alpha} \cap U_{\beta}\right)$ tal que

$$
Z_{\beta}=h \circ Z_{\alpha} \text { em } U_{\alpha} \cap U_{\beta}
$$

Logo,

$$
L\left(Z_{\beta}\right)=L\left(h \circ Z_{\alpha}\right)=0 \text { em } U_{\alpha} \cap U_{\beta},
$$

pois $L\left(Z_{\alpha}\right)=0$ em $U_{\alpha} \cap U_{\beta}$ e $h$ é holomorfa em $Z_{\alpha}\left(U_{\alpha} \cap U_{\beta}\right)$. Em particular, $L\left(Z_{\beta}\right)\left(p_{0}\right)=0$.

Por outro lado, como $h$ é um biholomorfismo, a aplicação

$$
\tilde{h}\left(y_{1}, y_{2}\right) \doteq\left(\Re\left\{h\left(y_{1}+i y_{2}\right)\right\}, \Im\left\{h\left(y_{1}+i y_{2}\right)\right\}\right),\left(y_{1}, y_{2}\right) \in F_{\alpha}\left(U_{\alpha} \cap U_{\beta}\right)
$$

é um difeomorfismo. Temos ainda que

$$
\left(\Re\left\{Z_{\beta}\right\}, \Im\left\{Z_{\beta}\right\}\right)=\left(\Re\left\{h \circ Z_{\alpha}\right\}, \Im\left\{h \circ Z_{\alpha}\right\}\right)=\tilde{h} \circ F_{\alpha} \text { em } U_{\alpha} \cap U_{\beta}
$$


Logo,

$$
J\left(\tilde{h} \circ F_{\alpha}\right)(p)=\left[\begin{array}{cc}
\frac{\partial \Re\left\{Z_{\beta}\right\}}{\partial x_{1}}(p) & \frac{\partial \Re\left\{Z_{\beta}\right\}}{\partial x_{2}}(p) \\
\frac{\partial \Im\left\{Z_{\beta}\right\}}{\partial x_{1}}(p) & \frac{\partial \Im\left\{Z_{\beta}\right\}}{\partial x_{2}}(p)
\end{array}\right]
$$

para $p \in U_{\alpha} \cap U_{\beta}$. Como $\tilde{h} \circ F_{\alpha}$ é um difeomorfismo definido em $U_{\alpha} \cap U_{\beta}$, temos que $\operatorname{det}\left(J\left(\tilde{h} \circ F_{\alpha}\right)\right) \neq 0$ em $U_{\alpha} \cap U_{\beta}$. Logo,

$$
\left(\frac{\partial \Re\left\{Z_{\beta}\right\}}{\partial x_{1}}(p), \frac{\partial \Re\left\{Z_{\beta}\right\}}{\partial x_{2}}(p)\right),\left(\frac{\partial \Im\left\{Z_{\beta}\right\}}{\partial x_{1}}(p), \frac{\partial \Im\left\{Z_{\beta}\right\}}{\partial x_{2}}(p)\right)
$$

são linearmente independentes em $U_{\alpha} \cap U_{\beta}$. Ou seja, $\operatorname{grad}\left(\Re\left\{Z_{\beta}\right\}\right), \operatorname{grad}\left(\Im\left\{Z_{\beta}\right\}\right)$ são linearmente independentes em $U_{\alpha} \cap U_{\beta}$. Em particular, $\operatorname{grad}\left(\Re\left\{Z_{\beta}\right\}\right)\left(p_{0}\right), \operatorname{grad}\left(\Im\left\{Z_{\beta}\right\}\right)\left(p_{0}\right)$ são linearmente independentes. Pela arbitrariedade de $p_{0} \in U_{\beta}$, temos que $L\left(Z_{\beta}\right)(p)=0$ e $\operatorname{grad}\left(\Re\left\{Z_{\beta}\right\}\right)(p), \operatorname{grad}\left(\Im\left\{Z_{\beta}\right\}\right)(p)$ são linearmente independentes para todo $p \in U_{\beta}$.

Assim fica provado o seguinte teorema:

Teorema 4.3.1. Seja $L$ um campo vetorial complexo elítico definido no aberto conexo $\Omega$ de $\mathbb{R}^{2}$. Então existe uma única estrutura diferenciável complexa $\mathscr{A}_{L}=\left\{\left(U_{\alpha}, Z_{\alpha}\right)\right\}_{\alpha \in \mathcal{I}}$ em $\Omega$ tal que

$\operatorname{grad}\left(\Re\left\{Z_{\alpha}\right\}\right), \operatorname{grad}\left(\Im\left\{Z_{\alpha}\right\}\right)$ são linearmente independentes em $U_{\alpha}$ e $L Z_{\alpha}=0$ em $U_{\alpha}$ para todo $\alpha \in \mathcal{I}$.

A estrutura $\mathscr{A}_{L}$ é chamada de estrutura diferenciável complexa induzida em $\Omega$ por $L$. 


\subsection{Solução do Problema}

Vamos usar os teoremas de uniformização de superfícies de Riemann discutidos no capítulo 2 para obter a solução do problema $\left(*^{\prime}\right)$, i.e., vamos mostrar o seguinte teorema:

Teorema 4.4.1. Seja L um campo vetorial complexo elítico definido em um aberto conexo $\Omega \subset \mathbb{R}^{2}$ onde $\pi_{1}(\Omega)=\{0\}$ ou $\pi_{1}(\Omega)=\mathbb{Z}$. Então o problema

$\left(*^{\prime}\right) \quad L Z=0, \operatorname{grad}(\Re\{Z\}), \operatorname{grad}(\Im\{Z\})$ linearmente independentes e $Z$ injetiva em $\Omega$ tem solução global.

\section{Demonstração:}

Seja $\mathscr{A}_{L}$ a estrutura diferenciável complexa definida em $\Omega$ por $L$. Seja $\left(\Omega, \mathscr{A}_{L}\right)$ a superfície de Riemann dada pelo conjunto $\Omega$ com a estrutura $\mathscr{A}_{L}$.

Primeiro suponha que $\Omega$ é um aberto simplesmente conexo do $\mathbb{R}^{2}$. Assim $\left(\Omega, \mathscr{A}_{L}\right)$ é uma superfície de Riemann simplesmente conexa. Pelo Teorema 2.2.2, $\left(\Omega, \mathscr{A}_{L}\right)$ é biholomorfa a uma das seguintes superfícies de Riemann: $\overline{\mathbb{C}}, \mathbb{C}$ e $\mathbb{D}$. Como $\overline{\mathbb{C}}$ é compacta e $\Omega$ é um aberto de $\mathbb{R}^{2}$, temos que $\overline{\mathbb{C}}$ e $\left(\Omega, \mathscr{A}_{L}\right)$ não são biholomorfos. Assim $\left(\Omega, \mathscr{A}_{L}\right)$ é biholomorfa a $\mathbb{C}$ ou $\mathbb{D}$. Logo, existe uma função holomorfa e injetiva de $\left(\Omega, \mathscr{A}_{L}\right)$ em $\mathbb{C}$. Agora, suponha que $\pi_{1}(\Omega)=\mathbb{Z}$. Pelo teorema 2.2.3, $\left(\Omega, \mathscr{A}_{L}\right)$ é biholomorfa a uma das seguintes superfícies de Riemann: $\mathbb{C}^{*}, \mathbb{D}^{*}$ e $A_{r}$. Logo, existe uma função holomorfa e injetiva de $\left(\Omega, \mathscr{A}_{L}\right)$ em $\mathbb{C}$. Portanto, se $\pi_{1}(\Omega)=\{0\}$ ou $\pi_{1}(\Omega)=\mathbb{Z}$, temos que existe

$$
Z: \Omega \longrightarrow \mathbb{C}
$$

tal que

$$
h_{\alpha} \doteq Z \circ Z_{\alpha}^{-1}: Z_{\alpha}\left(U_{\alpha}\right) \longrightarrow \mathbb{C}
$$

é holomorfa injetora para toda $\alpha \in \mathcal{I}$. Logo, para cada $\alpha \in \mathcal{I}$, a restrição de $Z$ a $U_{\alpha}$ pode ser escrita na forma

$$
\left.Z\right|_{U_{\alpha}}=h_{\alpha} \circ Z_{\alpha} \text { em } U_{\alpha}
$$


Em particular, segue que $\left.Z\right|_{U_{\alpha}} \in C^{\infty}\left(U_{\alpha}\right)$ para todo $\alpha \in \mathcal{I}$. Como $\left\{U_{\alpha}\right\}_{\alpha \in \mathcal{I}}$ é uma cobertura aberta de $\Omega$, temos que $Z \in C^{\infty}(\Omega)$. Segue de contas análogas às do final da seção anterior que $\operatorname{grad}(\Re\{Z\}), \operatorname{grad}(\Im\{Z\})$ são linearmente independentes em $U_{\alpha}$ para todo $\alpha \in \mathcal{I}$. Em particular, $d Z \neq 0$ em $U_{\alpha}$ para todo $\alpha \in \mathcal{I}$. Logo, $d Z \neq 0$ em $\Omega$.

Como $L Z_{\alpha}=0$ em $U_{\alpha}$ e $h_{\alpha}$ é holomorfa em $Z_{\alpha}\left(U_{\alpha}\right)$ para todo $\alpha \in \mathcal{I}$, temos para cada $\alpha \in \mathcal{I}$

$$
L Z(p)=L\left(h_{\alpha} \circ Z_{\alpha}\right)(p)=0 \text {, para todo } p \in U_{\alpha} .
$$

Pela arbitrariedade de $\alpha \in \mathcal{I}$ e pelo fato de $\left\{U_{\alpha}\right\}_{\alpha \in \mathcal{I}}$ ser uma cobertura aberta de $\Omega$,

$$
L Z=0 \text { em } \Omega \text {. }
$$

Portanto, $Z$ é solução do problema.

Corolário 4.4.2. Seja L um campo vetorial complexo elítico definido em um aberto conexo $\Omega \subset \mathbb{R}^{2}$ onde $\pi_{1}(\Omega)=\{0\}$ ou $\pi_{1}(\Omega)=\mathbb{Z}$. Então existe um difeomorfismo $F: \Omega \longrightarrow F(\Omega)$, tal que

$$
L_{F}=\lambda_{F} \frac{\partial}{\partial \bar{w}} \operatorname{em} F(\Omega)
$$

onde $w=y_{1}+i y_{2},\left(y_{1}, y_{2}\right) \in F(\Omega)$ e $\lambda_{F} \in C^{\infty}(F(\Omega))$ com $\lambda_{F}\left(y_{1}, y_{2}\right) \neq 0$, para todo $\left(y_{1}, y_{2}\right) \in F(\Omega)$.

\section{Demonstração:}

Seja $Z$ a solução do problema $\left(*^{\prime}\right)$. Observe que $F \doteq(\Re\{Z\}, \Im\{Z\})$ é um difeomorfismo do aberto $\Omega$ sobre o aberto $F(\Omega)$, onde $F(\Omega)=\left\{\left(y_{1}, y_{2}\right) \in \mathbb{R}^{2} ; y_{1}+i y_{2} \in Z(\Omega)\right\}$. De fato, para cada $p \in \Omega$, existe $\alpha \in \mathcal{I}$ tal que $p \in U_{\alpha}$ e $F_{\alpha}$ tal que $f_{\alpha} \doteq F \circ F_{\alpha}^{-1}$ : $F_{\alpha}\left(U_{\alpha}\right) \longrightarrow F\left(U_{\alpha}\right)$ é um difeomorfismo. Como $F_{\alpha}: U_{\alpha} \longrightarrow F_{\alpha}\left(U_{\alpha}\right)$ é um difeomorfismo, temos $\left.F\right|_{U_{\alpha}}=f_{\alpha} \circ F_{\alpha}: U_{\alpha} \longrightarrow F\left(U_{\alpha}\right)$ é um difeomorfismo. Pela arbitrariedade de $p \in \Omega$ e pelo fato de $F$ ser uma bijeção de $\Omega$ sobre $F(\Omega)$, temos o resultado.

Analogamente à demonstração do colorolário 4.2.8, escrevendo $L$ nas novas coordenadas induzidas por $F$ temos 


$$
L_{F}=\left(L(\bar{Z}) \circ F^{-1}\right) \frac{1}{2}\left(\frac{\partial}{\partial y_{1}}+i \frac{\partial}{\partial y_{2}}\right)=\lambda \frac{\partial}{\partial \bar{w}}
$$

onde $w=y_{1}+i y_{2}$ e $\lambda \doteq L(\bar{Z}) \circ F^{-1}$ em $F(\Omega)$. Como a eliticidade é invariante por mudança de coordenadas, segue que $\lambda\left(y_{1}, y_{2}\right) \neq 0, \forall\left(y_{1}, y_{2}\right) \in F(\Omega)$. Ou seja, existe uma mudança de coordenadas global em $\Omega$ que torna $L$ um múltiplo do operador de Cauchy-Riemann.

Do teorema 4.4.1 podemos obter soluções para o problema $\left(*^{\prime}\right)$ no caso semiglobal. Mais especificamente:

Corolário 4.4.3. Seja L um campo elítico definido no aberto conexo $\Omega$ de $\mathbb{R}^{2}$. Então, para todo $\Omega^{\prime} \subset \overline{\Omega^{\prime}} \subset \Omega$ aberto existe $\mathscr{Z}: \Omega^{\prime} \longrightarrow \mathbb{C}$ injetiva com $\operatorname{grad}(\Re\{\mathscr{Z}\})(p)$, $\operatorname{grad}(\Im\{\mathscr{Z}\})(p)$, linearmente independentes para todo $p \in \Omega^{\prime}$ e $L \mathscr{Z}=0$ em $\Omega^{\prime}$.

\section{Demonstração:}

Como foi visto anteriormente, podemos escrever

$$
L=\lambda\left(\frac{\partial}{\partial x_{2}}+(f+i g) \frac{\partial}{\partial x_{1}}\right)
$$

onde $f, g, \lambda \in C^{\infty}(\Omega)$ com $\lambda(p) \neq 0$, para todo $p \in \Omega$ e $f, g$ funções a valores reais com $g(p) \neq 0$, para todo $p \in \Omega$. Como estamos estudando as soluções de $L$, podemos considerar, sem perda de generalidade, que

$$
L=\frac{\partial}{\partial x_{2}}+(f+i g) \frac{\partial}{\partial x_{1}} \text { em } \Omega
$$

Também podemos supor, sem perda, que $g(p)>0$, para todo $p \in \Omega$.

Agora, seja $\Omega^{\prime} \subset \overline{\Omega^{\prime}} \subset \Omega$ um aberto. Como $\mathbb{R}^{2}$ é normal, existe um aberto $V$ de $\mathbb{R}^{2}$ tal que $\overline{\Omega^{\prime}} \subset V$ e $\bar{V} \subset \Omega$. Pelo lema de Urysohn existe uma função $\chi \in C^{\infty}\left(\mathbb{R}^{2}\right)$ tal que $\chi \equiv 1$ em $\overline{\Omega^{\prime}}, \chi \equiv 0$ em $V^{c}$, e $0 \leq \chi \leq 1$. Daí, defina

$$
\tilde{g}(p)=\left\{\begin{array}{rll}
(g(p)-1) \chi(p)+1 & \text { se } & p \in \Omega \\
1, & \text { se } & p \in \Omega^{c}
\end{array} \quad, \quad \tilde{f}(p)=\left\{\begin{array}{rll}
f(p) \chi(p) & \text { se } & p \in \Omega \\
0, & \text { se } & p \in \Omega^{c}
\end{array} .\right.\right.
$$


Temos que $\tilde{f}, \tilde{g} \in C^{\infty}\left(\mathbb{R}^{2}\right)$ e $\left.\tilde{f}\right|_{\Omega^{\prime}}=f,\left.\tilde{g}\right|_{\Omega^{\prime}}=g$ em $\Omega^{\prime}$. De fato, temos que a restrição de $\tilde{f}$ a $\Omega$ é a função $\left.(g-1) \chi\right|_{\Omega}+1$, que é uma função $C^{\infty}$. E a restrição de $\tilde{f}$ a $\mathbb{R}^{2} \backslash \bar{V}$ é identicamente 0 , que é uma função $C^{\infty}$. Como $\mathbb{R}^{2}=\Omega \cup\left(\mathbb{R}^{2} \backslash \bar{V}\right)$, temos que $f$ é de classe $C^{\infty}$ em $\mathbb{R}^{2}$. De forma análoga, mostramos que $\tilde{f}$ é $C^{\infty}$ em $\mathbb{R}^{2}$. É fácil ver que $\left.\tilde{g}\right|_{\Omega^{\prime}}=g$ e $\left.\tilde{f}\right|_{\Omega^{\prime}}=f$.

Agora considere

$$
\tilde{L}=\frac{\partial}{\partial x_{2}}+(\tilde{f}+i \tilde{g}) \frac{\partial}{\partial x_{1}} \text { em } \mathbb{R}^{2}
$$

Temos que $\tilde{L}$ é um campo em $\mathbb{R}^{2}$, mais ainda, $\tilde{L}$ é elítico em $\mathbb{R}^{2}$. Basta observar que $\tilde{g}(p) \neq$ 0 , para todo $p \in \mathbb{R}^{2}$. Como $\mathbb{R}^{2}$ é um aberto simplesmente conexo, pelo teorema 4.4.1 existe $Z: \mathbb{R}^{2} \longrightarrow \mathbb{C}$ injetiva com $\operatorname{grad}(\Re\{Z\})(p), \operatorname{grad}(\Im\{Z\})(p)$ linearmente independentes para todo $p \in \mathbb{R}^{2}$, com $\tilde{L} Z=0$ em $\mathbb{R}^{2}$. Observe que $\left.\tilde{L}\right|_{\Omega^{\prime}}=L$. Assim, seja $\left.\mathscr{Z} \doteq Z\right|_{\Omega^{\prime}}$. Daí, temos que $L \mathscr{Z}=0$ em $\Omega^{\prime}$ e satisfaz a propriedades desejadas.

Observe que não impomos condições sobre a topologia de $\Omega^{\prime}$.

Um comentário que pode ser feito a respeito do corolário acima é o seguinte: seja $L$ um campo elítico definido em um aberto $\Omega$ de $\mathbb{R}^{2}$. Se existe um campo elítico $M$ definido em aberto conexo $U$ de $\mathbb{R}^{2}$ com $\Omega \subset \bar{\Omega} \subset U$ e $\left.M\right|_{\Omega}=L$, então o problema (*') (para o campo $L)$ tem solução global. Note que a solução é obtida independe do grupo fundamental de $\Omega$.

Uma consequência do corolário acima é seguite resultado:

Corolário 4.4.4. Seja $L$ um campo elítico definido no aberto conexo $\Omega$ de $\mathbb{R}^{2}$. Então, para todo $\Omega^{\prime} \subset \overline{\Omega^{\prime}} \subset \Omega$ aberto existe um difeomorfismo $F: \Omega^{\prime} \longrightarrow F\left(\Omega^{\prime}\right)$, tal que

$$
L_{F}=\lambda_{F} \frac{\partial}{\partial \bar{w}} \operatorname{em} F\left(\Omega^{\prime}\right)
$$

onde $w=y_{1}+i y_{2},\left(y_{1}, y_{2}\right) \in F\left(\Omega^{\prime}\right)$ e $\lambda_{F} \in C^{\infty}\left(F\left(\Omega^{\prime}\right)\right)$ com $\lambda_{F}\left(y_{1}, y_{2}\right) \neq 0$, para todo $\left(y_{1}, y_{2}\right) \in F\left(\Omega^{\prime}\right)$. 
Demonstração: A demonstração deste corolário é análoga a demonstração do corolário 4.4.2. 


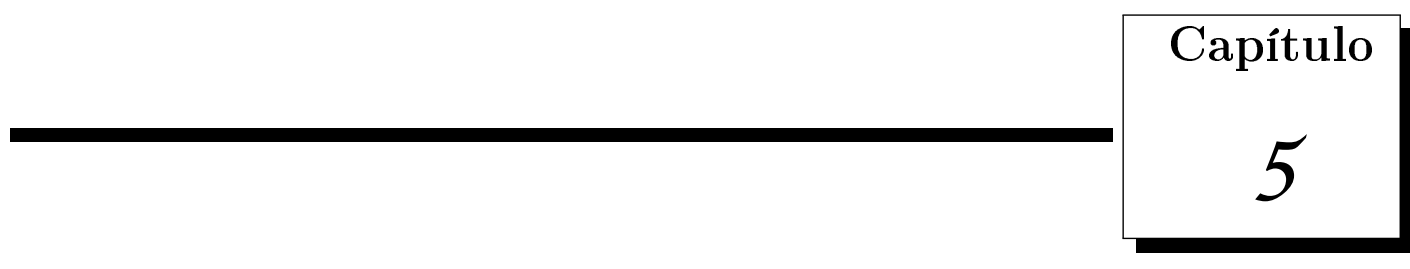

\section{Caso Hipoelítico}

\subsection{Introdução}

Neste capítulo vamos mostrar que se $L$ é um campo vetorial complexo não singular e hipoelítico definido em um aberto conexo $\Omega \subset \mathbb{R}^{2}$ onde $\pi_{1}(\Omega)=\{0\}$ ou $\pi_{1}(\Omega)=\mathbb{Z}$, então o problema

$$
\text { (*) } \quad L Z=0, d Z \neq 0 \text { e } Z \text { injetiva em } \Omega
$$

tem solução global.

Para obter a solução de $(*)$ vamos estudar a estrutura complexa induzida em $\Omega$ por um campo vetorial complexo hipoelítico não singular $L$ definido em $\Omega$.

\subsection{Hipoeliticidade e Integrabilidade Local}

De modo semelhante a um campo vetorial complexo elítico, um campo hipoelítico definido em um aberto do plano possui uma caracterização em função da integrabilidade local. O objetivo desta seção é demonstrar o teorema enunciado a seguir. 
Teorema 5.2.1. Seja L um campo vetorial complexo não singular definido no aberto $\Omega \subset \mathbb{R}^{2}$. As seguintes afirmações são equivalentes:

\section{L é hipoelítico em $\Omega$;}

2. existe uma família $\left\{\left(U_{\alpha}, Z_{\alpha}\right)\right\}_{\alpha \in J}$ tal que $\left\{U_{\alpha}\right\}_{\alpha \in J}$ é uma cobertura aberta de $\Omega$ e para cada $\alpha \in J$, existe $Z_{\alpha}: U_{\alpha} \longrightarrow \mathbb{C}$ função de classe $C^{\infty}$ tal que $L Z_{\alpha}=0$, $d Z_{\alpha} \neq 0$ e injetiva em $U_{\alpha}$.

\section{Demonstração:}

A demonstração será feita nas próximas 15 páginas e será realizada por meio de várias observações e lemas. Vamos começar mostrando que $1 \Longrightarrow 2$.

Como queremos provar um resultado local, seja $p_{0} \in \Omega$ um ponto. Pelo corolário 3.5.3 existe uma função $Z_{V}: V \longrightarrow \mathbb{C}$, onde $V$ é uma vizinhança aberta de $p_{0}$ em $\Omega$, tal que $L Z_{V}=0$ (em particular, $Z_{V} \in C^{\infty}(V)$, pois $L$ é hipoelítico) e $d Z_{V} \neq 0$ em $V$. Pelo lema 1.5.28 existe $F: U \longrightarrow W_{2}$ um difeomorfismo $C^{\infty}$, com o aberto $U \subset V$ e $W_{2}=(-\epsilon, \epsilon) \times(-\epsilon, \epsilon), \epsilon>0$, e um polinômio $P$ de grau 1 tal que

$$
\left(P \circ Z_{V}\right) \circ F^{-1}=x+i \phi(x, t),(x, t) \in W_{2},
$$

onde $\phi$ é a valores reais e $\phi(0,0)=0$. Note que a composição de $P$ com $Z_{V}$ não altera as boas propriedades de $Z_{V}$, i.e., $L\left(P \circ Z_{V}\right)=0$ e $d\left(P \circ Z_{V}\right) \neq 0$ em $V$. Definimos $\tilde{Z_{U}}$ como a restrição de $P \circ Z_{V}$ ao aberto $U$. Temos que $Z \doteq F^{*}\left(Z_{U}\right)=\tilde{Z}_{U} \circ F^{-1}$ é solução do campo $L_{F}$ em $W_{2}$. Lembremos que $L_{F}$ é hipoelítico em $W_{2}$. Como foi visto, a hipoeliticidade é invariante por mudança de coordenadas. Portanto, é suficiente mostrar o resultado para $Z$, pois a composição $C^{\infty}$, i.e., a mudança de variáveis, preserva as propriedades desejadas. Ou seja, se $Z: W_{2} \longrightarrow \mathbb{C}$ é uma aplicação aberta e injetiva, então $Z_{U}=F^{-1^{*}}(Z): U \longrightarrow \mathbb{C}$ é injetiva e aberta. Portanto, o trabalho que se segue nesta seção, é voltado ao estudo da função

$$
Z: W_{2} \longrightarrow \mathbb{C} \text { que satisfaz } L_{F} Z=0 \text { e } d Z \neq 0 \text { em } W_{2} \text {. }
$$


O primeiro passo é mostrar que a hipoeliticidade de $L_{F}$ implica que $Z$ é uma aplicação aberta. E veremos, que o fato de $Z$ ser aplicação aberta, está estreitamente relacionado com sua injetividade.

Sejam $p=\left(p_{1}, p_{2}\right) \in \mathbb{R}^{2}$ e $\rho_{1}, \rho_{2}>0$. Definimos

$$
R_{\rho_{1} \rho_{2}}(p) \doteq\left\{(x, t) \in \mathbb{R}^{2} ;\left|x-p_{1}\right|<\rho_{1} \text { e }\left|t-p_{2}\right|<\rho_{2}\right\}
$$

Note que $R_{\rho_{1} \rho_{2}}(p)$ é um aberto do $\mathbb{R}^{2}$.

Obs. 5.2.2. A imagem $Z\left(R_{\rho_{1} \rho_{2}}(p)\right)$ de $R_{\rho_{1} \rho_{2}}(p) \subset W_{2}$ por $Z$, é união de segmentos verticais. Mais precisamente,

$$
Z\left(R_{\rho_{1} \rho_{2}}(p)\right)=\bigcup_{x \in\left(p_{1}-\rho_{1}, p_{1}+\rho_{1}\right)}\left(x+i J\left(p, \rho_{2}\right)_{x}\right)
$$

onde

$$
J\left(p, \rho_{2}\right)_{x}=\left\{\phi(x, t) ; t \in\left(p_{2}-\rho_{2}, p_{2}-\rho_{2}\right)\right\} .
$$

Obs. 5.2.3. $L_{F}$ hipoelítico, então para cada $R_{\rho_{1} \rho_{2}}(p) \subset W_{2}, J\left(p, \rho_{2}\right)_{x}$ é um intervalo com interior não vazio para todo $x \in\left(p_{1}-\rho_{1}, p_{1}+\rho_{1}\right)$. De fato, suponha que existe $x_{0} \in\left(p_{1}-\rho_{1}, p_{1}+\rho_{1}\right)$ tal que $J\left(p, \rho_{2}\right)_{x_{0}}=t_{0}$, isto é, $\left(p_{2}-\rho_{2}, p_{2}-\rho_{2}\right) \ni t \longmapsto \phi\left(x_{0}, t\right)$ é constante. Logo $\partial_{t} \phi\left(x_{0}, t\right)=0$ para todo $t \in\left(p_{2}-\rho_{2}, p_{2}-\rho_{2}\right)$. Agora observe que a menos de um fator $C^{\infty}$ que não se anula em $W_{2}$,

$$
L_{F}=\left(1+i \partial_{x} \phi\right)\left\{\frac{\partial}{\partial t}-\left(\frac{i \partial_{t} \phi}{1+i \partial_{x} \phi}\right) \frac{\partial}{\partial x}\right\}
$$

em $W_{2}$. Assim temos que o coeficiente que acompanha a derivada parcial em relação a $x$ se anula na curva $\alpha(s)=\left(x_{0}, s\right), s \in\left(p_{2}-\rho_{2}, p_{2}-\rho_{2}\right)$. Segue do exemplo 3.4 .1 que $L_{F}$ não é hipoelítico em $R_{\rho_{1} \rho_{2}}(p)$.

Concluímos assim que $J\left(p, \rho_{2}\right)_{x_{0}}$ não é um intervalo degenerado, i.e., int $\left(J\left(p, \rho_{2}\right)_{x_{0}}\right) \neq$ $\emptyset$.

Lema 5.2.4. $Z\left(R_{\rho_{1} \rho_{2}}(p)\right)$ é aberto se, e somente se, $J\left(p, \rho_{2}\right)_{x}$ é um intervalo aberto para todo $x \in\left(p_{1}-\rho_{1}, p_{1}+\rho_{1}\right)$. 


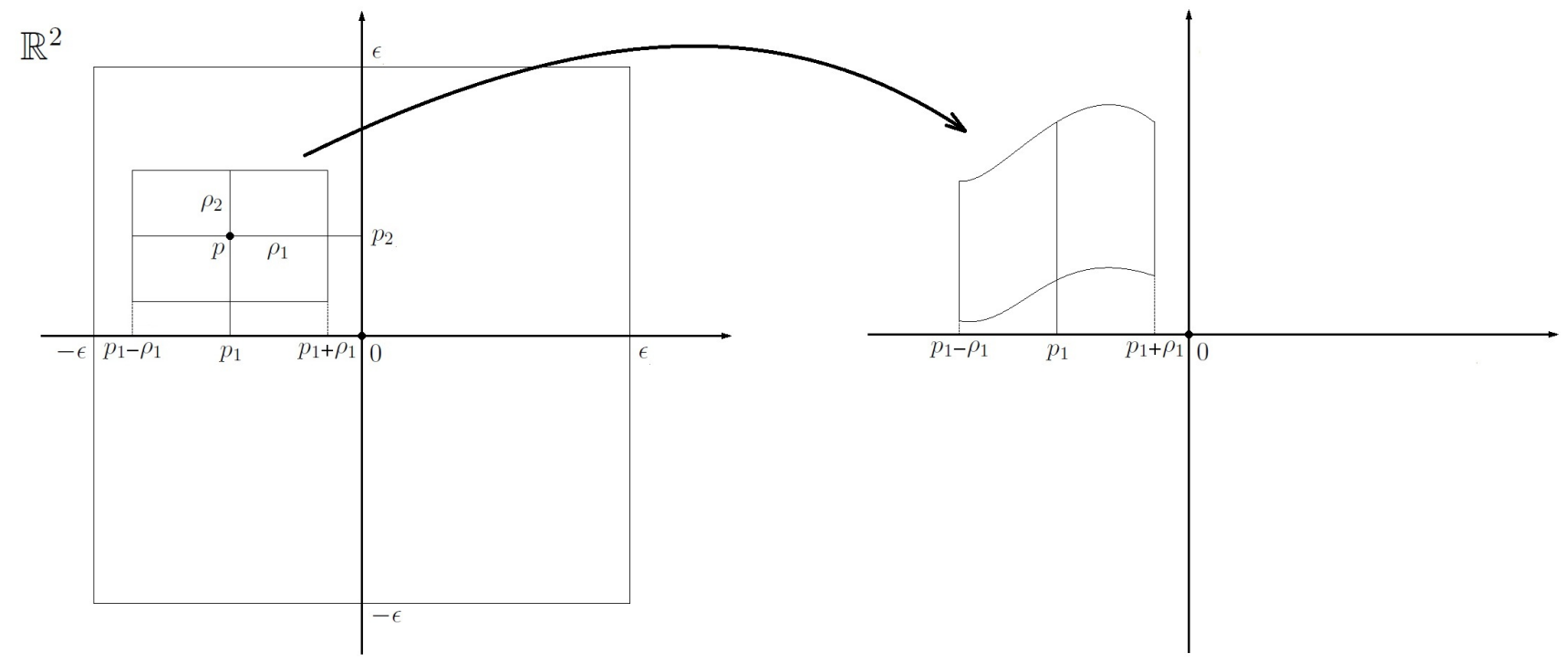

\section{Demonstração:}

Primeiramente suponhamos $Z\left(R_{\rho_{1} \rho_{2}}(p)\right)$ seja aberto. Seja $x \in\left(p_{1}-\rho_{1}, p_{1}+\rho_{1}\right)$. Considere a reta $r=\{w \in \mathbb{C} ; \Re\{w\}=x\}$ com a topologia induzida de $\mathbb{C}$. Como $Z\left(R_{\rho_{1} \rho_{2}}(p)\right)$ é um aberto de $\mathbb{C}$, temos que $r \cap Z\left(R_{\rho_{1} \rho_{2}}(p)\right)=\left\{x+i \phi(x, t) ; t \in\left(p_{2}-\rho_{2}, p_{2}+\rho_{2}\right)\right\}$ é um aberto de $r$. Temos também que $f: r \longrightarrow \mathbb{R}$ dada por $f(w)=\Im\{w\}$ é um homeomorfismo. Além disso, $f\left(r \cap Z\left(R_{\rho_{1} \rho_{2}}(p)\right)\right)=J\left(p, \rho_{2}\right)_{x}$. Portanto, $J\left(p, \rho_{2}\right)_{x}$ é um aberto. Como $\phi$ é contínua, temos que

$$
\left(p_{2}-\rho_{2}, p_{2}+\rho_{2}\right) \ni t \mapsto \phi(x, t)
$$

é contínua, logo $J\left(p, \rho_{2}\right)_{x}$ é conexo pois $J\left(p, \rho_{2}\right)_{x}=\phi\left(x,\left(p_{2}-\rho_{2}, p_{2}+\rho_{2}\right)\right)$ e $\left(p_{2}-\rho_{2}, p_{2}+\rho_{2}\right)$ é conexo. Portanto, $J\left(p, \rho_{2}\right)_{x}$ é um intervalo aberto.

Reciprocamente, seja $q=\left(q_{1}, q_{2}\right) \in R_{\rho_{1} \rho_{2}}(p)$. Vamos mostrar que $Z(q)$ é ponto interior de $Z\left(R_{\rho_{1} \rho_{2}}(p)\right)$. Temos que $Z(q)=q_{1}+i \phi\left(q_{1}, q_{2}\right)$. Por hipótese, temos que $J\left(p, \rho_{2}\right)_{q_{1}}$ é um intervalo aberto, segue daí que existem $t^{(1)}, t^{(2)} \in\left(p_{2}-\rho_{2}, p_{2}+\rho_{2}\right)$ tais que

$$
\phi\left(q_{1}, t^{(1)}\right)<\phi\left(q_{1}, q_{2}\right)<\phi\left(q_{1}, t^{(2)}\right)
$$

Como $\phi$ é contínua, segue que a aplicação 


$$
\left(p_{1}-\rho_{1}, p_{1}+\rho_{1}\right) \ni x \mapsto \phi\left(x, q_{2}\right)
$$

é contínua. Logo existe $\epsilon>0$ tal que $\left(q_{1}-\epsilon, q_{1}+\epsilon\right) \subset\left(p_{1}-\rho_{1}, p_{1}+\rho_{1}\right)$ e

$$
\phi\left(x, t^{(1)}\right)<\phi\left(q_{1}, q_{2}\right)<\phi\left(x, t^{(2)}\right)
$$

para todo $x \in\left(q_{1}-\epsilon, q_{1}+\epsilon\right)$. Temos também que

$$
x \mapsto \phi\left(x, t^{(j)}\right)
$$

é contínua $(j=1,2)$ e como $\left[q_{1}-\epsilon / 2, q_{1}+\epsilon / 2\right]$ é compacto, existem $x^{(1)}, x^{(2)} \in\left[q_{1}-\right.$ $\left.\epsilon / 2, q_{1}+\epsilon / 2\right]$ tais que

$$
\phi\left(x, t^{(1)}\right) \leq \phi\left(x^{(1)}, t^{(1)}\right)<\phi\left(q_{1}, q_{2}\right)<\phi\left(x^{(2)}, t^{(2)}\right) \leq \phi\left(x, t^{(2)}\right)
$$

para todo $x \in\left[q_{1}-\epsilon / 2, q_{1}+\epsilon / 2\right]$.

Seja

$$
A=\left\{w \in \mathbb{C} ; \Re\{w\} \in\left(q_{1}-\epsilon / 2, q_{1}+\epsilon / 2\right) \text { e } \Im\{w\} \in\left(\phi\left(x^{(1)}, t^{(1)}\right), \phi\left(x^{(2)}, t^{(2)}\right)\right)\right\} .
$$

Temos que $A$ é aberto e $Z(q) \in A$. Além disso, de (5.2.1) temos

$$
\left(\phi\left(x^{(1)}, t^{(1)}\right), \phi\left(x^{(2)}, t^{(2)}\right)\right) \subset\left(\phi\left(x, t^{(1)}\right), \phi\left(x, t^{(2)}\right)\right) \subset J\left(p, \rho_{2}\right)_{x}
$$

para todo $x \in\left(q_{1}-\epsilon / 2, q_{1}+\epsilon / 2\right)$. Logo

$$
A \subset \bigcup_{x \in\left(q_{1}-\epsilon / 2, q_{1}+\epsilon / 2\right)}\left(x+i J\left(p, \rho_{2}\right)_{x}\right) \subset \bigcup_{x \in\left(p_{1}-\rho_{1}, p_{1}+\rho_{1}\right)}\left(x+i J\left(p, \rho_{2}\right)_{x}\right)=Z\left(R_{\rho_{1} \rho_{2}}(p)\right) .
$$

Assim concluímos que $Z(q)$ é ponto interior de $Z\left(R_{\rho_{1} \rho_{2}}(p)\right)$. Pela arbitrariedade de $q \in$ $R_{\rho_{1} \rho_{2}}(p)$, segue que $Z\left(R_{\rho_{1} \rho_{2}}(p)\right)$ é aberto.

Lema 5.2.5. Se $L_{F}$ é hipoelítico, então $\forall p \in W_{2}, J\left(p, \rho_{2}\right)_{x}$ é um intervalo aberto para todo $x \in\left(p_{1}-\rho_{1}, p_{1}+\rho_{1}\right)$.

Demonstração: Prova por contradição. 
Suponha que $R_{\rho_{1} \rho_{2}}(p) \subset W_{2}$ é tal que existe $x_{0} \in\left(p_{1}-\rho_{1}, p_{1}+\rho_{1}\right)$ tal que $J\left(p, \rho_{2}\right)_{x_{0}}$ não é um intervalo aberto.

Sem perda de generalidade podemos supor que

$$
J\left(p, \rho_{2}\right)_{x_{0}} \subset\{z ; \Re\{z\}=0 \text { e } \Im\{z\} \leq 0\}
$$

e também que $y_{0} \doteq \sup \left\{\phi\left(x_{0}, t\right) ; t \in\left(p_{2}-\rho_{2}, p_{2}-\rho_{2}\right)\right\} \in J\left(p, \rho_{2}\right)_{x_{0}}$, i.e., $J\left(p, \rho_{2}\right)_{x_{0}}$ é fechado superiormente.

Seja

$$
h(z)=|z|^{\frac{3}{2}} \exp \left(i \frac{3}{2} \operatorname{Arg}(z)\right), z \in U_{0}=\mathbb{C} \backslash\{z ; \Re\{z\}=0 \text { e } \Im\{z\} \geq 0\}
$$

onde $A r g$ é o ramo do argumento definido em $U_{0}$; explicitamente, Arg: $U_{0} \longrightarrow \mathbb{R}$ é dado por $\operatorname{Arg}(z)=\theta$, onde $\theta$ satisfaz $-\frac{3 \pi}{2}<\theta<\frac{\pi}{2}$ e $z=|z| e^{i \theta}$.

Obs. 5.2.6. h é holomorfa em $U_{0}$, mais especificamente, $h$ é o ramo de $z^{\frac{3}{2}}$ cujo corte é $\{z ; \Re\{z\}=0$ e $\Im\{z\} \geq 0\}$. Em particular,

$$
|h(z)|=|z|^{\frac{3}{2}},\left|h^{\prime}(z)\right|=\frac{3}{2}|z|^{\frac{1}{2}} \text { e }\left|h^{\prime \prime}(z)\right|=\frac{3}{4}|z|^{-\frac{1}{2}} \text {, para } z \in U_{0} .
$$

Além disso, definindo $h(0)=0, h$ é contínua em $U_{0} \cup\{0\}$. Outra propriedade de $h$ é que ela não se estende de forma holomorfa, a um aberto que contém 0, i.e., não existe uma função holomorfa $\tilde{h}: \tilde{U}_{0} \longrightarrow \mathbb{C}$, tal que $\tilde{U}_{0}$ é um aberto de $\mathbb{C}$ com $U_{0} \cup\{0\} \subset \tilde{U}_{0}$ e $\left.\tilde{h}\right|_{U_{0}}=h$ em $U_{0}$. Estas propriedades são a chave da demonstração deste lema.

Considere

$$
T(z) \doteq z-\left(x_{0}+i y_{0}\right), z \in \mathbb{C}
$$

Seja

$$
\Sigma=\left\{(x, t) \in R_{\rho_{1} \rho_{2}}(p) ; Z(x, t)=x_{0}+i y_{0}\right\}
$$

Pela observação 5.2.6 temos que a composta $(h \circ T) \circ Z$ está bem definida no aberto $R_{\rho_{1} \rho_{2}}(p) \backslash \Sigma$. Mais ainda, temos que $h \circ T$ é analítica em $\mathbb{C} \backslash\left\{z ; \Re\{z\}=x_{0}\right.$ e $\left.\Im\{z\} \geq y_{0}\right\}$ 
e a imagem de $R_{\rho_{1} \rho_{2}}(p) \backslash \Sigma$ por $Z$ está contida em $\mathbb{C} \backslash\left\{z ; \Re\{z\}=x_{0}\right.$ e $\left.\Im\{z\} \geq y_{0}\right\}$. Definimos

$$
u(x, t)=\left\{\begin{array}{rll}
((h \circ T) \circ Z)(x, t), & \text { se } & (x, t) \in R_{\rho_{1} \rho_{2}}(p) \backslash \Sigma \\
0, & \text { se } & (x, t) \in \Sigma .
\end{array}\right.
$$

Pelo comentário acima é fácil ver que $u \in C^{\infty}$ em $R_{\rho_{1} \rho_{2}}(p) \backslash \Sigma$ e que $L_{F} u=0$ em $R_{\rho_{1} \rho_{2}}(p) \backslash \Sigma$.

Obs. 5.2.7. u é contínua, pois é composta de funções contínuas.

Afirmamos que $u$ é $C^{1}$ em $R_{\rho_{1} \rho_{2}}(p)$. De fato, seja $\left(x_{0}, t_{0}\right) \in \Sigma$. Para $(x, t) \in R_{\rho_{1} \rho_{2}}(p)$ e $s \in \mathbb{R} \backslash\{0\}$ temos

$$
\begin{aligned}
& \left|\frac{u\left(x_{0}+s, t_{0}\right)-u\left(x_{0}, t_{0}\right)}{s}\right|=\left|\frac{u\left(x_{0}+s, t_{0}\right)}{s}\right|=\left|\frac{s^{2}+\left(\phi\left(x_{0}+s, t_{0}\right)-y_{0}\right)^{2}}{s^{\frac{4}{3}}}\right|^{\frac{3}{4}} \\
= & \left|s^{\frac{2}{3}}+s^{\frac{2}{3}}\left(\frac{\phi\left(x_{0}+s, t_{0}\right)-y_{0}}{s}\right)^{2}\right|^{\frac{3}{4}}=|s|^{\frac{1}{2}}\left|1+\left(\frac{\phi\left(x_{0}+s, t_{0}\right)-\phi\left(x_{0}, t_{0}\right)}{s}\right)^{2}\right|^{\frac{3}{4}} .
\end{aligned}
$$

Assim

$$
\left|\frac{u\left(x_{0}+s, t_{0}\right)-u\left(x_{0}, t_{0}\right)}{s}\right| \longrightarrow 0, \text { quando } s \longrightarrow 0
$$

Portanto $\frac{\partial u}{\partial x}\left(x_{0}, t_{0}\right)=0$. Analogamente,

$$
\begin{gathered}
\left|\frac{u\left(x_{0}, t_{0}+s\right)-u\left(x_{0}, t_{0}\right)}{s}\right|=\left|\frac{h\left(i\left(\phi\left(x_{0}, t_{0}+s\right)-y_{0}\right)\right)}{s}\right|=\frac{\left|\phi\left(x_{0}, t_{0}+s\right)-y_{0}\right|^{\frac{3}{2}}}{|s|} \\
=\left|\frac{\phi\left(x_{0}, t_{0}+s\right)-y_{0}}{s^{\frac{2}{3}}}\right|^{\frac{3}{2}}=|s|^{\frac{1}{2}}\left|\frac{\phi\left(x_{0}, t_{0}+s\right)-\phi\left(x_{0}, t_{0}\right)}{s}\right|^{\frac{3}{2}} .
\end{gathered}
$$

Assim

$$
\left|\frac{u\left(x_{0}, t_{0}+s\right)-u\left(x_{0}, t_{0}\right)}{s}\right| \longrightarrow 0, \text { quando } s \longrightarrow 0
$$

Portanto $\frac{\partial u}{\partial t}\left(x_{0}, t_{0}\right)=0$.

Pela arbitrariedade de $\left(x_{0}, t_{0}\right) \in \Sigma$, Temos que $\frac{\partial u}{\partial x}=\frac{\partial u}{\partial t} \equiv 0$ em $\Sigma$.

Seja $(x, t) \in R_{\rho_{1} \rho_{2}}(p) \backslash \Sigma$. Segue da proposição 1.5.30 que 
- $\frac{\partial u}{\partial x}(x, t)=(h \circ T)^{\prime}(Z(x, t)) \cdot \frac{\partial Z}{\partial x}(x, t)=\left(h^{\prime} \circ T\right)(Z(x, t)) \cdot \frac{\partial Z}{\partial x}(x, t)$

$$
=h^{\prime}\left(x+i \phi(x, t)-\left(x_{0}+i y_{0}\right)\right)\left(1+i \frac{\partial \phi}{\partial x}(x, t)\right)
$$

- $\frac{\partial u}{\partial t}(x, t)=(h \circ T)^{\prime}(Z(x, t)) \cdot \frac{\partial Z}{\partial x}(x, t)=\left(h^{\prime} \circ T\right)(Z(x, t)) \cdot \frac{\partial Z}{\partial t}(x, t)$

$$
=h^{\prime}\left(x+i \phi(x, t)-\left(x_{0}+i y_{0}\right)\right)\left(i \frac{\partial \phi}{\partial t}(x, t)\right)
$$

em $R_{\rho_{1} \rho_{2}}(p) \backslash \Sigma$. Da observação 5.2.6 segue que

$$
\frac{\partial u}{\partial x}(x, t) \longrightarrow 0 \mathrm{e} \frac{\partial u}{\partial t}(x, t) \longrightarrow 0
$$

quando $(x, t) \longrightarrow\left(x_{0}, t_{0}\right),(x, t) \in R_{\rho_{1} \rho_{2}}(p) \backslash \Sigma$. Portanto $\frac{\partial u}{\partial x}$ e $\frac{\partial u}{\partial t}$ são contínuas em $U$. Segue daí que $u \in C^{1}\left(R_{\rho_{1} \rho_{2}}(p)\right)$.

Considerando $u$ como uma distribuição em $R_{\rho_{1} \rho_{2}}(p)$, temos que $L_{F} u=0$ no sentido das distribuições em $R_{\rho_{1} \rho_{2}}(p)$. De fato, temos que $L_{F} u$ é uma função contínua, uma vez que podemos olhar para $L_{F}$ como um operador diferencial parcial linear de ordem 1 e $u \in C^{1}\left(R_{\rho_{1} \rho_{2}}(p)\right)$. Por outro lado, $L_{F} u$ restrita ao aberto $R_{\rho_{1} \rho_{2}}(p) \backslash \Sigma$ é identicamente nula, ou seja, $\operatorname{supp}\left(L_{F} u\right) \subset J\left(p, \rho_{2}\right)_{x_{0}}$. Como $J\left(p, \rho_{2}\right)_{x_{0}}$ tem medida nula, segue que $L_{F} u=0$.

No entanto, $u \notin C^{2}\left(R_{\rho_{1} \rho_{2}}(p)\right)$. De fato, para $(x, t) \in R_{\rho_{1} \rho_{2}}(p) \backslash \Sigma$ temos

$$
\begin{aligned}
& \frac{\partial^{2} u}{\partial x^{2}}(x, t)=\left(h^{\prime \prime} \circ T\right)(Z(x, t)) \cdot\left[\frac{\partial Z}{\partial x}(x, t)\right]^{2}+\left(h^{\prime} \circ T\right)(Z(x, t)) \cdot \frac{\partial^{2} Z}{\partial x^{2}}(x, t) \\
& \quad=\left(h^{\prime \prime} \circ T\right)(Z(x, t)) \cdot\left(1+i \frac{\partial \phi}{\partial x}(x, t)\right)^{2}+\left(h^{\prime} \circ T\right)(Z(x, t)) \cdot\left(i \frac{\partial^{2} \phi}{\partial x^{2}}(x, t)\right) .
\end{aligned}
$$

Pela observação 5.2.6, temos que

- $\left(h^{\prime \prime} \circ T\right)(Z(x, t)) \cdot\left(1+i \frac{\partial \phi}{\partial x}(x, t)\right)^{2} \longrightarrow \infty$,

quando $(x, t) \longrightarrow\left(x_{0}, t_{0}\right),(x, t) \in U \backslash \Sigma$. 
- $\left(h^{\prime} \circ T\right)(Z(x, t)) \cdot\left(i \frac{\partial^{2} \phi}{\partial x^{2}}(x, t)\right) \longrightarrow 0$,

quando $(x, t) \longrightarrow\left(x_{0}, t_{0}\right),(x, t) \in R_{\rho_{1} \rho_{2}}(p) \backslash \Sigma$.

Portanto,

$$
\frac{\partial^{2} u}{\partial x^{2}}(x, t) \longrightarrow \infty
$$

quando $(x, t) \longrightarrow\left(x_{0}, t_{0}\right),(x, t) \in R_{\rho_{1} \rho_{2}}(p) \backslash \Sigma$. Logo $u \notin C^{2}\left(R_{\rho_{1} \rho_{2}}(p)\right)$. Chegamos assim, em contradição com o fato de $L$ ser hipoelítico em $U$, pois $L u \in C^{\infty}\left(R_{\rho_{1} \rho_{2}}(p)\right)$, mas $u \notin C^{\infty}\left(R_{\rho_{1} \rho_{2}}(p)\right)$.

Lema 5.2.8. Se $L_{F}$ é hipoelítico, então $Z: W_{2} \longrightarrow \mathbb{C}$ é uma aplicação aberta.

Demonstração: Observe que

$Z$ é aberta $\Longleftrightarrow \forall R_{\rho_{1} \rho_{2}}(p) \subset U$, o conjunto $Z\left(R_{\rho_{1} \rho_{2}}(p)\right)$ é um aberto do $\mathbb{R}^{2}$.

Suponha que $Z$ não é aberta. Então existem $p \in W_{2}$ e $\rho_{1}, \rho_{2}>0$ tais que $R_{\rho_{1} \rho_{2}}(p) \subset W_{2}$ e $Z\left(R_{\rho_{1} \rho_{2}}(p)\right)$ não é um aberto do $\mathbb{R}^{2}$. Logo, pelo lema 5.2.4, existe $x_{0} \in\left(p_{1}-\rho_{1}, p_{1}+\rho_{1}\right)$ tal que $J\left(p, \rho_{2}\right)_{x_{0}}$ não é um intervalo aberto. E assim pelo lema 5.2.5, temos que $L_{F}$ não é hipoelítico.

Proposição 5.2.9. $Z: W_{2} \longrightarrow \mathbb{C}$, onde $W_{2}=(-\epsilon, \epsilon) \times(-\epsilon, \epsilon), \epsilon>0$, é aberta se, $e$ somente se, para cada $x \in(-\epsilon, \epsilon)$, a função

$$
(-\epsilon, \epsilon) \ni t \longmapsto \phi(x, t)
$$

é injetiva, onde $\phi$ é uma função a valores reais tal que $Z(x, t)=x+i \phi(x, t)$ em $W_{2}$.

Demonstração: Vamos provar por contrapositiva. 
Suponha que existe $x_{0} \in(-\epsilon, \epsilon)$ tal que a função $(-\epsilon, \epsilon) \ni t \longmapsto \phi\left(x_{0}, t\right)$ não é injetiva. Assim existem $t_{1}, t_{2} \in(-\epsilon, \epsilon) \operatorname{com} t_{1}<t_{2}$, tais que $\phi\left(x_{0}, t_{1}\right)=\phi\left(x_{0}, t_{2}\right)$. Seja $p_{0}=\left(x_{0}, t_{0}\right)$, onde $t_{0}=\left(t_{1}+t_{2}\right) / 2$. E seja também $\lambda=\left|\left(t_{1}-t_{2}\right) / 2\right|$.

Sejam $p_{M}$ um ponto de máximo e $p_{m}$ um ponto mínimo de $\phi\left(x_{0}, \cdot\right)$ em $\left[t_{1}, t_{2}\right]$. Vamos analisar alguns casos:

1. $p_{M}=p_{m}=t_{1}$

Então $\phi\left(x_{0}, \cdot\right)$ é constante em $\left[t_{1}, t_{2}\right]$. Logo $J\left(p_{0}, \lambda\right)_{x_{0}}=\left\{\phi\left(x_{0}, t_{0}\right)\right\}$ que não é um intervalo aberto.

2. $p_{M}=p_{m}=t_{2}$

Então $\phi\left(x_{0}, \cdot\right)$ é constante em $\left[t_{1}, t_{2}\right]$. Logo $J\left(p_{0}, \lambda\right)_{x_{0}}=\left\{\phi\left(x_{0}, t_{0}\right)\right\}$ que não é um intervalo aberto.

3. $p_{M}=t_{1}, p_{m} \neq t_{1}$ e $p_{M} \neq p_{m}$.

Temos neste caso que $p_{m} \in\left(t_{1}, t_{2}\right)$. E assim, o intervalo $J\left(p_{0}, \lambda\right)_{x_{0}}$ possui extremo inferior igual a $\phi\left(x_{0}, p_{m}\right)$. Logo $J\left(p_{0}, \lambda\right)_{x_{0}}$ não é aberto.

4. $p_{m}=t_{1}, p_{M} \neq t_{1}$ e $p_{M} \neq p_{m}$.

Temos neste caso que $p_{M} \in\left(t_{1}, t_{2}\right)$. E assim, o intervalo $J\left(p_{0}, \lambda\right)_{x_{0}}$ possui extremo superior igual a $\phi\left(x_{0}, p_{M}\right)$. Logo $J\left(p_{0}, \lambda\right)_{x_{0}}$ não é aberto.

5. $p_{M}, p_{m} \neq t_{1}, p_{M}, p_{m} \neq t_{2}\left(\mathrm{e} p_{M} \neq p_{m}\right)$. Neste caso $p_{M}, p_{m} \in\left(t_{1}, t_{2}\right)$. Logo $J\left(p, \rho_{2}\right)_{x_{0}}=\left[\phi\left(x_{0}, p_{m}\right), \phi\left(x_{0}, p_{m}\right)\right]$. Ou seja, $J\left(p_{0}, \lambda\right)_{x_{0}}$ não é um intervalo aberto.

Pelo lema 5.2.4, em todos os casos o conjunto $Z\left(R_{\epsilon \lambda}\left(p_{0}\right)\right)$ não é aberto. Portanto $Z$ não é aberta.

Reciprocamente, suponha que $Z$ não é aberta. Então existem $p \in W_{2}$ e $\rho_{1}, \rho_{2}>0$ tais que $R_{\rho_{1} \rho_{2}}(p) \subset U$ e $Z\left(R_{\rho_{1} \rho_{2}}(p)\right)$ não é um aberto do $\mathbb{R}^{2}$. Logo, pelo lema 5.2.4, existe $x_{0} \in\left(p_{1}-\rho_{1}, p_{1}+\rho_{1}\right)$ tal que $J\left(p, \rho_{2}\right)_{x_{0}}=\left\{\phi\left(x_{0}, t\right) ; t \in\left(p_{2}-\rho_{2}, p_{2}-\rho_{2}\right)\right\}$ 
não é um intervalo aberto. Assim $(-\epsilon, \epsilon) \ni t \longmapsto \phi\left(x_{0}, t\right)$ não é injetiva. De fato, se $\phi\left(x_{0}, \cdot\right)$ fosse injetiva, então $\phi\left(x_{0}, \cdot\right)$ seria um homeomorfismo sobre $\phi\left(x_{0},(-\epsilon, \epsilon)\right)$. Em particular, $\phi\left(x_{0}, \cdot\right)$ é aberta. E assim $\phi\left(x_{0},\left(p_{2}-\rho_{2}, p_{2}-\rho_{2}\right)\right)$ seria um intervalo aberto, ou seja, $J\left(p, \rho_{2}\right)_{x_{0}}$ seria aberto. Contradição. Portanto, existe $x_{0} \in(-\epsilon, \epsilon)$, tal que $(-\epsilon, \epsilon) \ni t \longmapsto \phi\left(x_{0}, t\right)$ não é injetiva.

Corolário 5.2.10. Se $L_{F}$ é hipoelítico, então para cada $x \in(-\epsilon, \epsilon)$,

$$
(-\epsilon, \epsilon) \ni t \longmapsto \phi(x, t)
$$

é uma função injetiva.

Demonstração: Segue direto do lema 5.2.8 e da proposição 5.2.9.

Obs. 5.2.11. $Z$ é injetiva em $W_{2}$ se, e somente se, para todo $x \in(-\epsilon, \epsilon)$ a função $(-\epsilon, \epsilon) \ni t \longmapsto \phi(x, t)$ é injetiva. De fato, suponha que $Z$ é injetiva. Dado $x \in(-\epsilon, \epsilon)$, sejam $t_{1}, t_{2} \in(-\epsilon, \epsilon)$ tais que $\phi\left(x, t_{1}\right)=\phi\left(x, t_{2}\right)$. Logo,

$$
i \phi\left(x, t_{1}\right)=i \phi\left(x, t_{1}\right) \Longrightarrow x+i \phi\left(x, t_{1}\right)=x+i \phi\left(x, t_{1}\right)
$$

Ou seja, $Z\left(x, t_{1}\right)=Z\left(x, t_{2}\right)$. Como $Z$ é injetiva, $\left(x, t_{1}\right)=\left(x, t_{2}\right)$ e assim $t_{1}=t_{2}$. Reciprocamente, suponha que para todo $x \in(-\epsilon, \epsilon)$ a função $(-\epsilon, \epsilon) \ni t \longmapsto \phi(x, t)$ é injetiva. Sejam $\left(x_{1}, t_{1}\right),\left(x_{2}, t_{2}\right) \in U$ tais que $Z\left(x_{1}, t_{1}\right)=Z\left(x_{2}, t_{2}\right)$. Logo

$$
\begin{aligned}
x_{1}+i \phi\left(x_{1}, t_{1}\right) & =x_{2}+i \phi\left(x_{2}, t_{2}\right) \Longleftrightarrow x_{1}=x_{2} \text { e } \phi\left(x_{1}, t_{1}\right)=\phi\left(x_{2}, t_{2}\right) \\
& \Longleftrightarrow x_{1}=x_{2} \text { e } \phi\left(x_{1}, t_{1}\right)=\phi\left(x_{1}, t_{2}\right) .
\end{aligned}
$$

Como $\phi\left(x_{1}, \cdot\right)$ é injetiva, temos $t_{1}=t_{2}$. Portanto $\left(x_{1}, t_{1}\right)=\left(x_{2}, t_{2}\right)$.

Corolário 5.2.12. Se $Z$ é aberta, então $Z$ é injetiva em $W_{2}$.

Demonstração: Segue diretamente da proposição 5.2.9 e da observação 5.2.11. 
Pela arbitrariedade de $p_{0} \in \Omega$, segue finalmente dos resultados acima que vale $1 \Rightarrow 2$ no teorema 5.2.1.

Obs. 5.2.13. Note que a familia $\left\{\left(U_{\alpha}, Z_{\alpha}\right)\right\}_{\alpha \in J}$ obtida acima, tem a seguinte propriedade: para cada $\alpha \in J$ a função $Z_{\alpha}: U_{\alpha} \longrightarrow \mathbb{C}$ é uma aplicação aberta.

Agora vamos mostrar $2 \Longrightarrow 1$.

Vamos mostrar que $L$ é hipoelítico em todo ponto de $\Omega$. Dado $p_{0} \in \Omega$, seja $\alpha \in J$ tal que $p_{0} \in U_{\alpha}$. Pelo lema 1.5.28 existe um difeomorfimo $\Phi: U \longrightarrow(-a, a) \times(-b, b), a, b>0$, com $U \subset U_{\alpha}$ vizinhança aberta de $p_{0}$, tal que

$$
Z \doteq\left(\Phi^{-1}\right)^{*}\left(\left.Z_{\alpha}\right|_{U}\right)(x, t)=x+i \phi(x, t)
$$

para $(x, t) \in R_{a b}(0)=(-a, a) \times(-b, b), a, b>0$, onde $\phi$ é uma função a valores reais com $\phi(0,0)=0$. Além disso,

$$
L_{\Phi} Z=0 \text { em } R_{a b}(0)
$$

Como $Z_{\alpha}: U_{\alpha} \longrightarrow \mathbb{C}$ é injetiva, segue que $Z: R_{a b}(0) \longrightarrow \mathbb{C}$ é injetiva. Segue da observação 5.2.11 que, para cada $x \in(-a, a)$ fixado, a função

$$
(-b, b) \ni t \longrightarrow \phi(x, t)
$$

é injetiva. Daí, para cada $x \in(-a, a)$ fixado, a função

$$
(-b, b) \ni t \longrightarrow \phi_{t}(x, t)
$$

não muda de sinal e não se anula em intervalos, i.e., para cada $x \in(-a, a)$, o conjunto $\Gamma_{x}=\left\{t \in(-b, b) ; \phi_{t}(x, t)=0\right\}$ não contém intervalos abertos. Então, os conjuntos

$$
A^{+}=\left\{x \in(-a, a) ; \sup _{t} \phi_{t}(x, t)>0\right\}
$$

e

$$
A^{-}=\left\{x \in(-a, a) ; \inf _{t} \phi_{t}(x, t)<0\right\}
$$


são abertos disjuntos, e o complementar da união, $F=(-a, a) \backslash\left(A^{+} \cup A^{-}\right)$é um conjunto fechado, com a propriedade de que $\phi_{t}(x, t)=0$ em $F \times(-b, b)$.

Note que $F$ é o conjunto vazio. De fato, se $F \neq \emptyset$, então existe $x_{0} \in F$ tal que $(-a, a) \ni t \longrightarrow \phi_{t}\left(x_{0}, t\right)$ é a função nula, ou seja, $\Gamma_{x_{0}}=(-b, b)$. Contradição.

Daí, concluímos que $R_{a b}(0)$ é união dos abertos disjuntos $A^{+} \times(-b, b)$ e $A^{-} \times(-b, b)$. Como $R_{a b}(0)$ é conexo, segue que $A^{+}=\emptyset$ ou $A^{-}=\emptyset$. Sem perda de generalidade, podemos supor que $A^{-}=\emptyset$. Assim, temos $A^{+}=(-a, a)$, i.e., $A^{+} \times(-b, b)=R_{a b}(0)$. Seja $x \in A^{+}$, então existe $t \in(-b, b)$ tal que $\sup _{t} \phi_{t}(x, t)>0$, e é fácil ver que $\phi_{t}(x, t) \geq 0 \mathrm{em}$ $A^{+} \times(-b, b)$. Ou seja, $\phi_{t}(x, t) \geq 0$ em $R_{a b}(0)$.

Temos que $L_{\Phi}=\lambda M$ em $R_{a b}(0)$, onde $\lambda \in C^{\infty}\left(R_{a b}(0)\right)$ com $\lambda(x, t) \neq 0$ para todo $(x, t) \in R_{a b}(0)$ e $M$ é um campo vetorial complexo em $R_{a b}(0)$ dado por

$$
M=\frac{\partial Z}{\partial x} \frac{\partial}{\partial t}-\frac{\partial Z}{\partial t} \frac{\partial}{\partial x}
$$

Logo,

$$
L_{\Phi}=\lambda\left(1+i \partial_{x} \phi\right) \frac{\partial}{\partial t}-i \partial_{t} \phi \frac{\partial}{\partial x}
$$

em $R_{a b}(0)$. Observe que $1+i \partial_{x} \phi(x, t) \neq 0$, para todo $(x, t) \in R_{a b}(0)$, logo podemos escrever

$$
\begin{aligned}
L_{\Phi} & =\lambda\left(1+i \partial_{x} \phi\right)\left\{\frac{\partial}{\partial t}-\left(\frac{i \partial_{t} \phi}{1+i \partial_{x} \phi}\right) \frac{\partial}{\partial x}\right\} \\
& =i \lambda\left(1+i \partial_{x} \phi\right)\left\{D_{t}-\left(\frac{i \partial_{t} \phi}{1+i \partial_{x} \phi}\right) D_{x}\right\}
\end{aligned}
$$

em $R_{a b}(0)$. Note que $i \lambda\left(1+i \partial_{x} \phi\right) \in C^{\infty}\left(R_{a b}(0)\right)$ e $\left[i \lambda\left(1+i \partial_{x} \phi\right)\right](x, t) \neq 0$, para todo $(x, t) \in R_{a b}(0)$. Seja

$$
N \doteq D_{t}-\left(\frac{i \partial_{t} \phi}{1+i \partial_{x} \phi}\right) D_{x} \text { em } R_{a b}(0)
$$

Logo, $L_{\Phi}$ é hipoelítico em $R_{a b}(0)$ se, e somente se, $N$ é hipoelítico em $R_{a b}(0)$. 
Agora, vamos verificar se $N$ satisfaz as condições (P) e (Q). Antes, note que podemos escrever

$$
N=D_{t}-\left(\left[\frac{i+\partial_{x} \phi}{1+\left(\partial_{x} \phi\right)^{2}}\right] \partial_{t} \phi\right) D_{x} \operatorname{em~} R_{a b}(0)
$$

Seja $\left(x_{0}, t_{0}\right) \in U_{2}$ e $\left(\xi_{10}, \xi_{20}\right) \in \mathbb{R}^{2} \backslash\{0\}$ tal que $N\left(\left(x_{0}, t_{0}\right),\left(\xi_{10}, \xi_{20}\right)\right)=0$. Então

$$
\xi_{2_{0}}-\left(\left[\frac{i+\partial_{x} \phi}{1+\left(\partial_{x} \phi\right)^{2}}\right] \partial_{t} \phi\right)\left(x_{0}, t_{0}\right) \xi_{1_{0}}=0
$$

o que equivale a

1. $\xi_{2_{0}}-\frac{\phi_{x}\left(x_{0}, t_{0}\right) \phi_{t}\left(x_{0}, t_{0}\right)}{1+\left(\phi_{x}\left(x_{0}, t_{0}\right)\right)^{2}} \xi_{1_{0}}=0$

2. $\frac{\phi_{t}\left(x_{0}, t_{0}\right)}{1+\left(\phi_{x}\left(x_{0}, t_{0}\right)\right)^{2}} \xi_{10}=0$.

Observe os dois fatos seguintes.

- Se $\phi_{t}\left(x_{0}, t_{0}\right) \neq 0$, então de 2 , temos $\xi_{10}=0$, e de 1 , temos $\xi_{20}=0$.

- Se $\phi_{t}\left(x_{0}, t_{0}\right)=0$, temos $\xi_{1_{0}} \in \mathbb{R} \backslash\{0\}$ e $\xi_{2_{0}}=0$.

Portanto, $N\left(\left(x_{0}, t_{0}\right),\left(\xi_{10}, \xi_{20}\right)\right)=0 \operatorname{com}\left(x_{0}, t_{0}\right) \in R_{a b}(0)$ e $\left(\xi_{10}, \xi_{2_{0}}\right) \in \mathbb{R}^{2} \backslash\{0\}$ implica $\left(x_{0}, t_{0}\right) \in R_{a b}(0)$ tal que $\phi_{t}\left(x_{0}, t_{0}\right)=0$ e $\left(\xi_{1_{0}}, \xi_{2_{0}}\right) \in \mathbb{R}^{2} \backslash\{0\}$ tal que $\xi_{1_{0}} \in \mathbb{R} \backslash\{0\}, \xi_{2_{0}}=0$.

Agora, seja $z \in \mathbb{C}$, tal que $d_{\xi} \Re\left\{z N\left(\left(x_{0}, t_{0}\right),\left(\xi_{1_{0}}, \xi_{2_{0}}\right)\right)\right\} \neq 0 . \operatorname{Dados}(x, t) \in R_{a b}(0)$ e $\left(\xi_{1}, \xi_{2}\right) \in \mathbb{R}^{2}$, temos com alguns cálculos,

$$
\Re\left\{z N\left((x, t),\left(\xi_{1}, \xi_{2}\right)\right)\right\}=\Re\{z\} \xi_{2}+\frac{\left(\Im\{z\}-\Re\{z\} \phi_{x}(x, t)\right)}{1+\left(\phi_{x}(x, t)\right)^{2}} \phi_{t}(x, t) \xi_{1}
$$

e

$$
\Im\left\{z N\left((x, t),\left(\xi_{1}, \xi_{2}\right)\right)\right\}=\Im\{z\} \xi_{2}-\frac{\left(\Re\{z\}+\Im\{z\} \phi_{x}(x, t)\right)}{1+\left(\phi_{x}(x, t)\right)^{2}} \phi_{t}(x, t) \xi_{1} .
$$

Assim

$$
d_{\xi} \Re\left\{z N\left((x, t),\left(\xi_{1}, \xi_{2}\right)\right)\right\}=\frac{\left(\Im\{z\}-\Re\{z\} \phi_{x}(x, t)\right)}{1+\left(\phi_{x}(x, t)\right)^{2}} \phi_{t}(x, t) d \xi_{1}+\Re\{z\} d \xi_{2} .
$$

Logo, 


$$
d_{\xi} \Re\left\{z N\left(\left(x_{0}, t_{0}\right),\left(\xi_{10}, \xi_{20}\right)\right)\right\}=0+\Re\{z\} d \xi_{2} .
$$

Portanto, para $\left(x_{0}, t_{0}\right),\left(\xi_{10}, \xi_{20}\right)$ como acima,

$$
d_{\xi} \Re\left\{z N\left(\left(x_{0}, t_{0}\right),\left(\xi_{10}, \xi_{20}\right)\right)\right\} \neq 0 \text { se, e somente se, } z \in \mathbb{C} \backslash\{z \in \mathbb{C} ; \Re\{z\}=0\} .
$$

Sejam $A(x, t) \doteq \frac{\left(\Im\{z\}-\Re\{z\} \phi_{x}(x, t)\right)}{1+\left(\phi_{x}(x, t)\right)^{2}}$ e $B(x, t)=-\frac{\left(\Re\{z\}+\Im\{z\} \phi_{x}(x, t)\right)}{1+\left(\phi_{x}(x, t)\right)^{2}},(x, t) \in$ $R_{a b}(0)$.

Seja $\alpha: I \longrightarrow R_{a b}(0) \times \mathbb{R}^{2}, \alpha(s)=\left((x(s), t(s)),\left(\xi_{1}(s), \xi_{2}(s)\right)\right)$, a curva bicaracterística nula de $\Re\{z N\}$ que passa por $\left(\left(x_{0}, t_{0}\right),\left(\xi_{10}, \xi_{20}\right)\right)$. Suponha que $0 \in I$ e $\alpha(0)=$ $\left(\left(x_{0}, t_{0}\right),\left(\xi_{1_{0}}, \xi_{20}\right)\right)$. Temos que $\alpha$ satisfaz:

1. $x^{\prime}(s)=A(x(s), t(s)) \phi_{t}(x(s), t(s))$;

2. $t^{\prime}(s)=\Re\{z\}$

3. $\xi_{1}^{\prime}(s)=-\xi_{1}(s) \partial_{x}\left(A \phi_{t}\right)(x(s), t(s))$;

4. $\xi_{2}^{\prime}(s)=-\xi_{1}(s) \partial_{t}\left(A \phi_{t}\right)(x(s), t(s)) . \quad(s \in I)$

Note que $\xi_{1}(s)=\xi_{10} e^{f(s)}, s \in I$, onde $f(s)=-\int_{0}^{s} \partial_{x}\left(A \phi_{t}\right)(x(r), t(r)) d r, s \in I$. Em particular, $\xi_{1}(s) \neq 0, \forall s \in I$. Note também que

$$
(\Re\{z N\} \circ \alpha)(s)=\Re\{z\} \xi_{2}(s)+A(x(s), t(s)) \phi_{t}(x(s), t(s)) \xi_{1}(s)=0, \forall s \in I .
$$

Logo,

$$
\xi_{2}(s)=-\frac{1}{\Re\{z\}} A(x(s), t(s)) \phi_{t}(x(s), t(s)) \xi_{1}(s), \forall s \in I .
$$

Agora vamos analisar $\Im\{z N\}$ restrita a $\alpha$, a curva bicaracterística nula de $\Re\{z L\}$ que passa por $\left(x_{0}, t_{0}\right)$.

$$
\begin{aligned}
& (\Im\{z N\} \circ \alpha)(s)=\Im\{z\} \xi_{2}(s)+B(x(s), t(s)) \phi_{t}(x(s), t(s)) \xi_{1}(s) \\
& =-\frac{\Im\{z\}}{\Re\{z\}} A(x(s), t(s)) \phi_{t}(x(s), t(s)) \xi_{1}(s)+B(x(s), t(s)) \phi_{t}(x(s), t(s)) \xi_{1}(s) \\
& =\left(-\frac{\Im\{z\}}{\Re\{z\}} A(x(s), t(s))+B(x(s), t(s))\right) \phi_{t}(x(s), t(s)) \xi_{1}(s)
\end{aligned}
$$


$=-\frac{|z|^{2}}{\Re\{z\}} \frac{\xi_{1}(s)}{1+\left(\phi_{x}(x(s), t(s))\right)^{2}} \phi_{t}(x(s), t(s)), \forall s \in I$.

Seja $C(s) \doteq-\frac{|z|^{2}}{\Re\{z\}} \frac{\xi_{1}(s)}{1+\left(\phi_{x}(x(s), t(s))\right)^{2}}, s \in I$.

Assim temos

$$
(\Im\{z N\} \circ \alpha)(s)=C(s) \phi_{t}(x(s), t(s)), \forall s \in I .
$$

Observe que $C(s) \neq 0, \forall s \in I$. Em particular, $C(s)$ não muda de sinal. Como $\phi_{t} \geq 0 \mathrm{em}$ $R_{a b}(0)$, segue que $N$ satisfaz a condição $(\mathrm{P})$.

Suponha por contradição que $N$ não satisfaça a condição $(Q)$ no ponto $\left(x_{0}, t_{0}\right)$. Então existe $\delta>0$ tal que $(\Im\{z L\} \circ \alpha)(s)=0$ para $s \in(-\delta, \delta) \subset I$. Logo, $\phi_{t}(x(s), t(s))=0$, $\forall s \in(-\delta, \delta)$, e assim, temos $x^{\prime}(s)=A(x(s), t(s)) \phi_{t}(x(s), t(s))=A(x(s), t(s)) \cdot 0=0$, $\forall s \in(-\delta, \delta)$. Daí,

$$
x(s)=x_{0}, \forall s \in(-\delta, \delta) .
$$

Como $t^{\prime}(s)=\Re\{z\}$ para $s \in I$, temos $t(s)=\Re\{z\} s+t_{0}, \forall s \in I$, logo

$$
\phi_{t}\left(x_{0}, \Re\{z\} s+t_{0}\right)=0, \forall s \in(-\delta, \delta) .
$$

Ou seja, $\Gamma_{x_{0}}$ contém o intervalo $\left(t_{0}-\Re\{z\} \delta, t_{0}+\Re\{z\} \delta\right)$. Contradição. Portanto $N$ satisfaz a condição (Q). Pelo teorema 3.4.8 temos que $N$ é hipoelítico, logo, $L_{\phi}$ é hipoelítico. Como hipoeliticidade é invariante por mudança de coordenadas, temos que $L$ é hipoelítico em $U$. Pela arbitrariedade de $p_{0} \in \Omega$, segue que $L$ é hipoelítico em $\Omega$.

Fica assim completa a demonstração do teorema 5.2.1. 


\subsection{Teorema de Aproximação e Estrutura das Soluções}

Essencialmente, nesta seção vamos tratar de um dos resultados mais importantes sobre campos vetoriais complexos localmente integráveis: O Teorema de Aproximação de Baouendi-Treves. Ele diz que dado qualquer ponto do domínio de um campo $L$ localmente integrável, existe uma vizinhança $W$ deste ponto tal que qualquer solução (distribucional) $L u=0$ nesta vizinhança pode ser aproximada por polinômios cuja "variável" é uma integral primeira do campo definida num aberto que contém $W$. Vamos demonstrar um caso particular deste teorema. Vamos demonstrar o caso em que a solução $u$ do campo, i.e., $L u=0$, é uma função $C^{\infty}$ no domínio de $L$. No entanto, este caso particular do teorema é suficiente para nosso propósito, uma vez que vamos usar o teorema de Baouendi-Treves para obter resultados sobre campos hipoelíticos.

\subsubsection{Teorema de Aproximação de Baouendi-Treves}

Seja $L$ um campo vetorial complexo localmente integrável definido no aberto $\Omega \subset \mathbb{R}^{2}$. $L$ ser localmente integrável, equivale a dizer que existe uma cobertura aberta $\mathcal{U}$ de $\Omega$, tal que para cada $U \in \mathcal{U}$, existe $Z_{U}: U \longrightarrow \mathbb{C}$ função $C^{\infty}$ que satisfaz $L Z_{U}=0$ e $d Z_{U} \neq 0$ em $U$.

Teorema 5.3.1. (Caso particular do Teorema de Baouendi-Treves). Seja $p \in \Omega$. Existe um par de abertos $U \in \mathcal{U}$ e $\subset \subset \Omega$, com $p \in W \subset \bar{W} \subset U \subset \Omega$, tal que se $u \in C^{\infty}(U)$ e satisfaz $L u=0$ em $U$, então existe uma sequência de polinômios $\left(P_{j}\right)_{j \in \mathbb{N}}$, tal que u é limite uniforme da sequência $P_{j}\left(Z_{U}\right)$ em $W$.

Exemplo 5.3.2. Considere o campo $L=\frac{\partial}{\partial t}$ definido no aberto $(a, b) \times(c, d), a, b>0$. É fácil ver que $Z(x, t)=x$ satisfaz $L Z=0$ e $d Z \neq 0$ em $(a, b) \times(c, d)$. Também é fácil ver que qualquer solução não constante da homogênea depende apenas da variável $x$. Dado $p=\left(p_{1}, p_{2}\right) \in(a, b) \times(c, d)$, seja $u \in C^{\infty}((a, b) \times(c, d))$ tal que Lu=0. Temos que u é da forma $u(x, t)=f(x),(x, t) \in(a, b) \times(c, d)$, com $f \in C^{\infty}((a, b))$. Pelo Teorema da Aproximação de Weierstrass existe uma vizinhança $V$ de $p_{1}$ em $(a, b)$ e uma sequência de polinômios na variável $x$ que converge uniformemente para $f$ em $V$. Segue dai que 
existe uma sequência de polinômios na "variável" Z que converge para u uniformemente em $V \times(c, d)$.

Exemplo 5.3.3. Seja L o operador de Cauchy-Riemann, i.e., o campo L é dado por

$$
L=\frac{1}{2}\left(\frac{\partial}{\partial x}+i \frac{\partial}{\partial t}\right), z=x+i t .
$$

Neste caso qualquer solução da homogênea é uma função holomorfa. É sabido da Análise Complexa que qualquer função holomofa localmente, pode ser aproximada uniformemente por polinômios na variável z.

\section{Demonstração do teorema 5.3.1:}

Vamos aplicar o lema 1.5 .28 a $Z_{U}$. Na mesma notação do lema, seja $\tilde{L}=L_{\Phi}$ o campo em $B$ induzido por $L$ via o difeomorfismo $\Phi^{-1}$. Sejam $a, T>0$ tais que $[-a, a] \times[-T, T] \subset B$. Definimos

$$
Z \doteq\left(Q \circ Z_{U} \circ \Phi^{-1}\right) \text { e } \tilde{u} \doteq\left(u \circ \Phi^{-1}\right)
$$

em $B$. Assim $Z$ e $\tilde{u}$ são soluções de homogênea em $B$, i.e., $L_{\Phi} Z=0$ e $L_{\Phi} \tilde{u}=0$. Por costume e para não sobrecarregar a notação, vamos usar as variáveis $x$ e $t$. Assim

$$
Z(x, t)=x+i \varphi(x, t)
$$

para $(x, t) \in[-a, a] \times[-T, T]$, onde $\varphi$ é uma função $C^{\infty}$ a valores reais que satisfaz

$$
\varphi(0,0)=\frac{\partial \varphi}{\partial x}(0,0)=0
$$

Além disso, reduzindo $a$ e $T$ se necessário podemos assumir que

$$
\left|\frac{\partial \varphi}{\partial x}(x, t)\right| \leq \frac{1}{2}, \text { para todo }(x, t) \in(-a, a) \times(-T, T)
$$

Seja $\chi \in C_{c}^{\infty}(B)$ tal que $\chi \geq 0, \chi \equiv 1$ em $(-a, a) \times(-T, T)$ e definamos $\phi \doteq \chi \varphi$. De agora em diante vamos considerar $Z(x, t)=x+i \phi(x, t),(x, t) \in \mathbb{R}^{2}$.

Seja $h \in C_{c}^{\infty}(\mathbb{R})$ tal que $\operatorname{supp}(h) \subset(-a, a)$ e $h \equiv 1$ em $(-a / 2, a / 2)$. Então fica bem definido o produto $\tilde{u}\left(x^{\prime}, 0\right) h\left(x^{\prime}\right)$ para $x^{\prime} \in \mathbb{R}$. Isto é importante para o inicio da demonstração, propriamente dito, que é definir uma família de funções $\left\{E_{\lambda} \tilde{u}\right\}$ com domínio $(-a, a) \times(-T, T)$ a um parâmetro $\lambda \in(0, \infty)$ pela fórmula 


$$
E_{\lambda} \tilde{u}(x, t)=\left(\frac{\lambda}{\pi}\right)^{\frac{1}{2}} \int_{\mathbb{R}} e^{-\lambda\left[Z(x, t)-Z\left(x^{\prime}, 0\right)\right]^{2}} \tilde{u}\left(x^{\prime}, 0\right) h\left(x^{\prime}\right) \frac{\partial Z}{\partial x}\left(x^{\prime}, 0\right) d x^{\prime}
$$

Como $e^{z}$ é inteira, é possível mostrar que $\tilde{L}\left(E_{\lambda} \tilde{u}\right)=0$ em $(-a, a) \times(-T, T)$, para todo $\lambda \in(0, \infty)$. Grosso modo, podemos dizer que $E_{\lambda}$ pode ser pensado como um operador que tem a imagem no espaço das soluções da homogênea.

A demonstração é dividida em duas etapas: a primeira é mostrar que $E_{\lambda} \tilde{u} \longrightarrow \tilde{u}$ uniformemente em uma vizinhança da origem, e a segunda é usar as propriedades analíticas da exponencial para obter a sequência de polinômios descrita no enunciado do teorema.

Para efetuar a primeira etapa da demonstração, considere a modificação do operador $E_{\lambda}$ dada por

$$
\begin{aligned}
& G_{\lambda} \tilde{u}(x, t)=\left(\frac{\lambda}{\pi}\right)^{\frac{1}{2}} \int_{\mathbb{R}} e^{-\lambda\left[Z(x, t)-Z\left(x^{\prime}, t\right)\right]^{2}} \tilde{u}\left(x^{\prime}, t\right) h\left(x^{\prime}\right) \frac{\partial Z}{\partial x}\left(x^{\prime}, t\right) d x^{\prime}, \\
& (x, t) \in(-a, a) \times(-T, T) .
\end{aligned}
$$

O primeiro passo é mostrar que $G_{\lambda} \tilde{u} \longrightarrow u$ uniformemente em uma vizinhança da origem quando $\lambda \longrightarrow \infty$.

Lema 5.3.4. Seja $z \in \mathbb{C} \operatorname{com} \Re\left\{z^{2}\right\}>0$, então $\int_{\mathbb{R}} e^{-(z x)^{2}} z d x=\pi^{\frac{1}{2}}$.

\section{Demonstração do lema:}

Seja $\Sigma=\left\{z \in \mathbb{C} ; \Re\left\{z^{2}\right\}>0\right\}$. Note que $\Sigma$ é um aberto de $\mathbb{C}$ e contém $\mathbb{R} \backslash\{0\}$. Inicialmente vamos estudar a convergência da integral.

$$
\left|\int_{\mathbb{R}} e^{-(z x)^{2}} z d x\right| \leq \int_{\mathbb{R}}\left|e^{-(z x)^{2}} z\right| d x \leq|z| \int_{\mathbb{R}} e^{-\Re\left\{z^{2}\right\} x^{2}} d x<\infty,
$$

para todo $z \in \Sigma$. Assim a função

$$
I(z)=\int_{\mathbb{R}} e^{-(z x)^{2}} z d x,
$$

para $z \in \Sigma$ fica bem definida, mais ainda, $I$ é holomorfa. De fato, segue do teorema da convergência dominada que

$$
\frac{\partial I}{\partial \bar{z}}(x+i y)=\int_{\mathbb{R}} \frac{\partial}{\partial \bar{z}}\left(e^{-\left((x+i y) x^{\prime}\right)^{2}}(x+i y)\right) d x^{\prime},
$$


para $z=x+i y \in \Sigma$. Como $z \mapsto e^{-(z x)^{2}} z, z \in \mathbb{C}$, é inteira, para todo $x^{\prime} \in \mathbb{R}$, segue que, $\frac{\partial I}{\partial \bar{z}}(x+i y)=0$, para todo $x+i y \in \Sigma$. Logo $I$ é holomorfa em $\Sigma$. É sabido do cálculo que

$$
\int_{\mathbb{R}} e^{-x^{2}} d x=\pi^{\frac{1}{2}}
$$

Através de uma simples mudança de variáveis na integral acima, concluímos

$$
\int_{\mathbb{R}} e^{-(t x)^{2}} t d x=\int_{\mathbb{R}} e^{-x^{2}} d x=\pi^{\frac{1}{2}}
$$

para $t \in \mathbb{R}$ e $t \neq 0$. Portanto $I$ é holomorfa em $\Sigma$ e é constante em $\mathbb{R} \backslash\{0\}$. Logo conclui se que $I$ é constante em $\Sigma$, i.e., $I(z)=\pi^{\frac{1}{2}}$, para todo $z \in \Sigma$. Assim, o lema esta provado.

De (5.3.1) temos

$$
\Re\left\{\left(\frac{\partial Z}{\partial x}(x, t)\right)^{2}\right\}=1-\left(\frac{\partial \phi}{\partial x}(x, t)\right)^{2}>0
$$

para cada $(x, t) \in(-a, a) \times(-T, T)$. Segue do lema acima que

$$
1=\pi^{-\frac{1}{2}} \int_{\mathbb{R}} e^{-\left(\frac{\partial Z}{\partial x}(x, t) x^{\prime}\right)^{2}} \frac{\partial Z}{\partial x}(x, t) d x^{\prime},
$$

para todo $(x, t) \in(-a, a) \times(-T, T)$. Daí,

$$
h(x) \tilde{u}(x, t)=\pi^{-\frac{1}{2}} \int_{\mathbb{R}} e^{-\left(\frac{\partial Z}{\partial x}(x, t) x^{\prime}\right)^{2}} v(x, t) d x^{\prime},
$$

em $(-a, a) \times(-T, T)$, onde $v(x, t)=h(x) \tilde{u}(x, t) \frac{\partial Z}{\partial x}(x, t)$. Note que o produto $h(x) \tilde{u}(x, t)$ fica bem definido para $(x, t) \in \mathbb{R} \times\{t ;|t| \leq T\}$, pelas boas propriedades de $h$.

Sejam fixados $\lambda>0$ e $(x, t) \in(-a, a) \times(-T, T)$. Fazendo a mudança de variáveis $x^{\prime}=x+\lambda^{-\frac{1}{2}} x^{\prime \prime}, x^{\prime \prime} \in \mathbb{R}$, obtemos

$$
\begin{aligned}
G_{\lambda} \tilde{u}(x, t) & =\left(\frac{\lambda}{\pi}\right)^{\frac{1}{2}} \int_{\mathbb{R}} e^{-\lambda\left[Z(x, t)-Z\left(x+\lambda^{-\frac{1}{2}} x^{\prime \prime}, t\right)\right]^{2}} v\left(x+\lambda^{-\frac{1}{2}} x^{\prime \prime}, t\right) \lambda^{-\frac{1}{2}} d x^{\prime \prime} \\
& =\pi^{-\frac{1}{2}} \int_{\mathbb{R}} e^{-\lambda\left[Z(x, t)-Z\left(x+\lambda^{-\frac{1}{2}} x^{\prime \prime}, t\right)\right]^{2}} v\left(x+\lambda^{-\frac{1}{2}} x^{\prime \prime}, t\right) d x^{\prime \prime} .
\end{aligned}
$$

Para não sobrecarregar a notação, voltamos a utilizar a variável de integração $x^{\prime}$. Assim 


$$
G_{\lambda} \tilde{u}(x, t)=\pi^{-\frac{1}{2}} \int_{\mathbb{R}} e^{-\lambda\left[Z(x, t)-Z\left(x+\lambda^{-\frac{1}{2}} x^{\prime}, t\right)\right]^{2}} v\left(x+\lambda^{-\frac{1}{2}} x^{\prime}, t\right) d x^{\prime},
$$

para todo $(x, t) \in(-a, a) \times(-T, T)$ e $\lambda>0$.

Então, para cada $\lambda>0$ podemos escrever

$$
G_{\lambda} \tilde{u}(x, t)-h(x) \tilde{u}(x, t)=I_{\lambda}(x, t)+J_{\lambda}(x, t)
$$

em $(-a, a) \times(-T, T)$, onde

$$
J_{\lambda}(x, t) \doteq \pi^{-\frac{1}{2}} \int_{\mathbb{R}}\left(e^{-\lambda\left[Z(x, t)-Z\left(x+\lambda^{-\frac{1}{2}} x^{\prime}, t\right)\right]^{2}}-e^{-\left(\frac{\partial Z}{\partial x}(x, t) x^{\prime}\right)^{2}}\right) v\left(x+\lambda^{-\frac{1}{2}} x^{\prime}, t\right) d x^{\prime}
$$

$\mathrm{e}$

$$
I_{\lambda}(x, t) \doteq \pi^{-\frac{1}{2}} \int_{\mathbb{R}} e^{-\left(\frac{\partial Z}{\partial x}(x, t) x^{\prime}\right)^{2}}\left(v\left(x+\lambda^{-\frac{1}{2}} x^{\prime}, t\right)-v(x, t)\right) d x^{\prime}
$$

\section{Estimativa de $I_{\lambda}$}

Seja $(x, t) \in(-a, a) \times(-T, T)$. Note que

$$
\begin{aligned}
\left|e^{-\left(\frac{\partial Z}{\partial x}(x, t) x^{\prime}\right)^{2}}\right| & =\left|e^{-\left(\left(1+i \frac{\partial \phi}{\partial x}(x, t)\right) x^{\prime}\right)^{2}}\right|=\left|e^{-\left(x^{\prime}+i \frac{\partial \phi}{\partial x}(x, t) x^{\prime}\right)^{2}}\right| \\
& =\left|e^{-x^{\prime 2}+x^{\prime 2} \frac{\partial \phi}{\partial x}(x, t)^{2}-2 x^{\prime 2} \frac{\partial \phi}{\partial x}(x, t) i}\right|=\left|e^{-x^{\prime 2}+x^{\prime 2} \frac{\partial \phi}{\partial x}(x, t)^{2}} e^{-2 x^{\prime 2} \frac{\partial \phi}{\partial x}(x, t) i}\right| \\
& =\left|e^{-x^{\prime 2}+x^{\prime 2} \frac{\partial \phi}{\partial x}(x, t)^{2}}\right|\left|e^{-2 x^{\prime 2} \frac{\partial \phi}{\partial x}(x, t) i}\right|=\left|e^{-x^{\prime 2}+x^{\prime 2} \frac{\partial \phi}{\partial x}(x, t)^{2}}\right| \\
& =e^{-x^{\prime 2}+x^{\prime 2} \frac{\partial \phi}{\partial x}(x, t)^{2}}=e^{-x^{\prime 2}+\left(\frac{\partial \phi}{\partial x}(x, t) x^{\prime}\right)^{2}} \leq e^{-x^{\prime 2}+x^{\prime 2} / 4} \\
& \leq e^{-3 x^{\prime 2} / 4}, \quad \forall x^{\prime} \in \mathbb{R} .
\end{aligned}
$$

Por outro lado, como $v$ se anula para $x \in\{x \in \mathbb{R} ;|x|>a\}$, temos que $\frac{\partial v}{\partial x}$ é limitado em $\mathbb{R} \times\{t ;|t| \leq T\}$. Ou seja, existe uma constante $C>0$ tal que

$$
\left|\frac{\partial v}{\partial x}(x, t)\right| \leq C, \text { para todo }(x, t) \in \mathbb{R} \times\{t ;|t| \leq T\} .
$$


Assim pelo teorema da desigualdade do valor médio

$$
\left|v\left(x+\lambda^{-\frac{1}{2}} x^{\prime}, t\right)-v(x, t)\right| \leq C \lambda^{-\frac{1}{2}}\left|x^{\prime}\right|
$$

para $x, x^{\prime} \in \mathbb{R}, t \in(-T, T)$ e $\lambda>0$.

Segue de (5.3.2) e (5.3.3) que para $(x, t) \in(-a, a) \times(-T, T)$

$$
\begin{aligned}
\left|I_{\lambda}(x, t)\right| & \leq \pi^{-\frac{1}{2}} \int_{\mathbb{R}}\left|e^{-\left(\frac{\partial Z}{\partial x}(x, t) x^{\prime}\right)^{2}}\left(v\left(x+\lambda^{-\frac{1}{2}} x^{\prime}, t\right)-v(x, t)\right)\right| d x^{\prime} \\
& \leq C \lambda^{-\frac{1}{2}}\left\{\pi^{-\frac{1}{2}} \int_{\mathbb{R}} e^{-3\left|x^{\prime}\right|^{2} / 4}\left|x^{\prime}\right| d x^{\prime}\right\}=\frac{4}{3} \pi^{-\frac{1}{2}} C \lambda^{-\frac{1}{2}}
\end{aligned}
$$

para todo $\lambda>0$. Isso mostra que $I_{\lambda} \longrightarrow 0$ uniformemente em $(-a, a) \times(-T, T)$ quando $\lambda \longrightarrow \infty$.

\section{Estimativa de $J_{\lambda}$}

Seja $R \geq 1$ uma constante. A ideia é estimar a integral da expressão de $J_{\lambda}$ em duas partes, para $\left|x^{\prime}\right|<R$ e $\left|x^{\prime}\right| \geq R$.

Lema 5.3.5. Uma função holomorfa num aberto $V \subset \mathbb{C}$, é lipschitziana em qualquer subconjunto convexo $X$ de $V$, onde sua derivada seja limitada.

\section{Demonstração do lema:}

Seja $f$ holomorfa em $V$ e sejam $z_{1}, z_{2} \in X$. Basta aplicar o Teorema da desigualdade do valor médio a curva $\alpha(t)=f\left(t z_{2}-(1-t) z_{1}\right), 0 \leq t \leq 1$.

Pelo lema existe uma constante $K>0$ tal que

$$
\left|e^{-z_{2}}-e^{-z_{1}}\right| \leq K\left|z_{2}-z_{1}\right|, \forall z_{1}, z_{2} \in H^{+}=\{z \in \mathbb{C} ; \Re\{z\} \geq 0\}
$$


Agora observe que para $(x, t) \in(-a, a) \times(-T, T), x^{\prime} \in \mathbb{R}$ e $\lambda>0$,

$$
\begin{aligned}
\Re\left\{\lambda\left[Z(x, t)-Z\left(x+\lambda^{-\frac{1}{2}} x^{\prime}, t\right)\right]^{2}\right\} & =\Re\left\{\lambda\left[i\left(\phi(x, t)-\phi\left(x+\lambda^{-\frac{1}{2}} x^{\prime}, t\right)\right)-\lambda^{-\frac{1}{2}} x^{\prime}\right]^{2}\right\} \\
& =\lambda\left[-\left(\phi(x, t)-\phi\left(x+\lambda^{-\frac{1}{2}} x^{\prime}, t\right)\right)^{2}+\lambda^{-1}\left(x^{\prime}\right)^{2}\right] \\
& \geq \lambda\left[-\frac{1}{4} \lambda^{-1}\left(x^{\prime}\right)^{2}+\lambda^{-1}\left(x^{\prime}\right)^{2}\right] \\
& =-\frac{1}{4}\left(x^{\prime}\right)^{2}+\left(x^{\prime}\right)^{2}=\frac{3}{4}\left(x^{\prime}\right)^{2} \geq 0 .
\end{aligned}
$$

Obs. 5.3.6. Foi usado acima, a desigualdade proveniente do teorema da desigualdade do valor médio e de (5.3.1):

$$
\begin{aligned}
\left|\phi(x, t)-\phi\left(x+\lambda^{-\frac{1}{2}} x^{\prime}, t\right)\right| \leq & \frac{1}{2} \lambda^{-\frac{1}{2}}\left|x^{\prime}\right|, \text { para } x, x^{\prime} \in \mathbb{R}, t \in(-T, T) \text { e } \lambda>0 . \\
\Re\left\{\left(\frac{\partial Z}{\partial x}(x, t) x^{\prime}\right)^{2}\right\} & =\Re\left\{x^{\prime 2}-x^{\prime 2} \frac{\partial \phi}{\partial x}(x, t)^{2}+2 x^{\prime 2} \frac{\partial \phi}{\partial x}(x, t) i\right\} \\
& =x^{\prime 2}-x^{\prime 2} \frac{\partial \phi}{\partial x}(x, t)^{2} \geq \frac{1}{2} x^{\prime 2} \geq 0,
\end{aligned}
$$

por (5.3.1).

Assim de (5.3.4), (5.3.5) e (5.3.6) temos

$$
\begin{gathered}
\left|e^{-\lambda\left[Z(x, t)-Z\left(x+\lambda^{-\frac{1}{2}} x^{\prime}, t\right)\right]^{2}}-e^{-\left(\frac{\partial Z}{\partial x}(x, t) x^{\prime}\right)^{2}}\right| \\
\leq K\left|\lambda\left[Z(x, t)-Z\left(x+\lambda^{-\frac{1}{2}} x^{\prime}, t\right)\right]^{2}-\left(\frac{\partial Z}{\partial x}(x, t) x^{\prime}\right)^{2}\right|,
\end{gathered}
$$

para $(x, t) \in(-a, a) \times(-T, T), x^{\prime} \in \mathbb{R}$ e $\lambda>0$. Agora, pela formula de Taylor,

$$
Z\left(x+\lambda^{-\frac{1}{2}} x^{\prime}, t\right)=Z(x, t)+\frac{\partial Z}{\partial x}(x, t) \lambda^{-\frac{1}{2}} x^{\prime}+A\left(x, t, x^{\prime}, \lambda\right)\left(\lambda^{-\frac{1}{2}} x^{\prime}\right)^{2},
$$

onde

$$
A\left(x, t, x^{\prime}, \lambda\right) \doteq 2 \int_{0}^{1}(1-s) \frac{\partial^{2} Z}{\partial x^{2}}\left(x+s \lambda^{-\frac{1}{2}} x^{\prime}, t\right) d s
$$


Note que $\operatorname{supp}\left(\frac{\partial^{2} Z}{\partial x^{2}}\right)$ é compacto, pois $\operatorname{supp}(\phi)$ é compacto. Em particular, existe $K_{1}>0$ tal que $\left|\frac{\partial^{2} Z}{\partial x^{2}}(x, t)\right| \leq K_{1}$, para todo $(x, t) \in \mathbb{R}^{2}$. Daí,

$$
\left|A\left(x, t, x^{\prime}, \lambda\right)\right| \leq K_{1}, \text { em }(-a, a) \times(-T, T) \times \mathbb{R} \times(0, \infty) .
$$

Assim, para $\left|x^{\prime}\right|<R$ temos

$$
\begin{gathered}
\left|\lambda\left[Z(x, t)-Z\left(x+\lambda^{-\frac{1}{2}} x^{\prime}, t\right)\right]^{2}-\left(\frac{\partial Z}{\partial x}(x, t) x^{\prime}\right)^{2}\right| \leq \\
\leq\left|A\left(x, t, x^{\prime}, \lambda\right)^{2}\left(x^{\prime}\right)^{4}\right| \lambda^{-1}+2\left|\frac{\partial Z}{\partial x}(x, t) A\left(x, t, x^{\prime}, \lambda\right)\left(x^{\prime}\right)^{3}\right| \lambda^{-\frac{1}{2}} \\
\leq K_{1}{ }^{2} R^{4} \lambda^{-1}+2 \frac{3}{2} K_{1} R^{3} \lambda^{-\frac{1}{2}} \leq K_{2} R^{4} \lambda^{-\frac{1}{2}}
\end{gathered}
$$

para $\lambda \geq 1$. Agora, para $\left|x^{\prime}\right| \geq R$ temos

$$
\begin{aligned}
\left|e^{-\lambda\left[Z(x, t)-Z\left(x+\lambda^{-\frac{1}{2}} x^{\prime}, t\right)\right]^{2}}-e^{-\left[\frac{\partial Z}{\partial x}(x, t) x^{\prime}\right]^{2}}\right| & =\left|e^{-\lambda\left[Z(x, t)-Z\left(x+\lambda^{-\frac{1}{2}} x^{\prime}, t\right)\right]^{2}}\right|+\left|e^{-\left[\frac{\partial Z}{\partial x}(x, t) x^{\prime}\right]^{2}}\right| \\
& \leq e^{\Re\left\{-\lambda\left[Z(x, t)-Z\left(x+\lambda^{-\frac{1}{2}} x^{\prime}, t\right)\right]^{2}\right\}}+\left|e^{-\left[\frac{\partial Z}{\partial x}(x, t) x^{\prime}\right]^{2}}\right| \\
& \leq 2 e^{-\frac{3}{4} x^{\prime 2}} \leq 2 e^{-\frac{3}{4}\left|x^{\prime}\right|} \\
& \leq 2 e^{-\frac{3}{4} R},
\end{aligned}
$$

pela estimativa (5.3.5) feita acima. Daí, para $(x, t) \in(-a, a) \times(-T, T)$

$$
\begin{gathered}
\left|J_{\lambda}(x, t)\right| \leq \\
\leq \pi^{-\frac{1}{2}} \int_{\mathbb{R}}\left|e^{-\lambda\left[Z(x, t)-Z\left(x+\lambda^{-\frac{1}{2}} x^{\prime}, t\right)\right]^{2}}-e^{-\left(\frac{\partial Z}{\partial x}(x, t) x^{\prime}\right)^{2}}\right|\left|v\left(x+\lambda^{-\frac{1}{2}} x^{\prime}, t\right)\right| d x^{\prime} \\
=\pi^{-\frac{1}{2}} \int_{\left\{x^{\prime} ;\left|x^{\prime}\right|<R\right\}}\left|e^{-\lambda\left[Z(x, t)-Z\left(x+\lambda^{-\frac{1}{2}} x^{\prime}, t\right)\right]^{2}}-e^{-\left(\frac{\partial Z}{\partial x}(x, t) x^{\prime}\right)^{2}}\right|\left|v\left(x+\lambda^{-\frac{1}{2}} x^{\prime}, t\right)\right| d x^{\prime} \\
+\pi^{-\frac{1}{2}} \int_{\left\{x^{\prime} ;\left|x^{\prime}\right| \geq R\right\}}\left|e^{-\lambda\left[Z(x, t)-Z\left(x+\lambda^{-\frac{1}{2}} x^{\prime}, t\right)\right]^{2}}-e^{-\left(\frac{\partial Z}{\partial x}(x, t) x^{\prime}\right)^{2}}\right|\left|v\left(x+\lambda^{-\frac{1}{2}} x^{\prime}, t\right)\right| d x^{\prime} \\
\leq 2 \pi^{-\frac{1}{2}} M K_{2} R^{5} \lambda^{-\frac{1}{2}}+\frac{16}{3} \pi^{-\frac{1}{2}} M e^{-\frac{3}{4} R}
\end{gathered}
$$




$$
\leq M_{1} K_{2} R^{5} \lambda^{-\frac{1}{2}}+M_{2} e^{-\frac{3}{4} R}, \text { para todo } \lambda \geq 1
$$

Segue que $J_{\lambda} \tilde{u} \longrightarrow 0$ uniformemente em $(-a, a) \times(-T, T)$ quando $\lambda \longrightarrow \infty$.

Assim concluímos o primeiro passo da demonstração, i.e., ficou provado que $G_{\lambda} \tilde{u} \longrightarrow \tilde{u}$ uniformemente em $(-a / 2, a / 2) \times(-T, T)$ quando $\lambda \longrightarrow \infty$.

Observe que não foi usado acima o fato de $Z$ e $\tilde{u}$ serem localmente soluções da homogênea (em relação a $\tilde{L}$ ). No entanto esta hipótese será essencial no próximo passo da demonstração, que é mostrar que $G_{\lambda} \tilde{u}-E_{\lambda} \tilde{u} \longrightarrow 0$ uniformemente em uma vizinhança da origem quando $\lambda \longrightarrow \infty$.

Sejam $(x, t) \in(-a / 2, a / 2) \times(-T, T)$ e $\lambda>0$ fixados. Considere a 1-forma complexa

$$
\omega\left(x^{\prime}, t^{\prime}\right)=V\left(x^{\prime}, t^{\prime}\right) \frac{\partial Z}{\partial x^{\prime}}\left(x^{\prime}, t^{\prime}\right) d x+V\left(x^{\prime}, t^{\prime}\right) \frac{\partial Z}{\partial t^{\prime}}\left(x^{\prime}, t^{\prime}\right) d t^{\prime}
$$

em $\mathbb{R} \times(-T, T)$, onde $V\left(x^{\prime}, t^{\prime}\right)=\left(\frac{\lambda}{\pi}\right)^{\frac{1}{2}} e^{-\lambda\left[Z(x, t)-Z\left(x^{\prime}, t^{\prime}\right)\right]^{2}} u\left(x^{\prime}, t^{\prime}\right) h\left(x^{\prime}\right)$.

Considere as curvas

$$
\begin{aligned}
& \gamma_{1}(s)=(a, t) s+(1-s)(-a, t) \\
& \gamma_{2}(s)=(a, 0) s+(1-s)(a, t) \\
& \gamma_{3}(s)=(-a, 0) s+(1-s)(a, 0) \\
& \gamma_{4}(s)=(-a, t) s+(1-s)(-a, 0)
\end{aligned}
$$

com $0 \leq s \leq 1$

Agora vamos computar as integrais de $\omega$ sobre as curvas descritas acima, nos cálculos vamos usar fortemente o fato de $V\left(x^{\prime}, t^{\prime}\right)=0$ para todo $\left(x^{\prime}, t^{\prime}\right)$ com $\left|x^{\prime}\right| \geq a$.

$$
\begin{aligned}
-\int_{\gamma_{1}} \omega & =\int_{0}^{1}\left[V\left(\gamma_{1}(s)\right) \frac{\partial Z}{\partial x^{\prime}}\left(\gamma_{1}(s)\right) 2 a+V\left(\gamma_{1}(s)\right) \frac{\partial Z}{\partial t^{\prime}}\left(\gamma_{1}(s)\right) 0\right] d s= \\
& =\int_{0}^{1} V(2 a s-a, t) \frac{\partial Z}{\partial x^{\prime}}(2 a s-a, t) 2 a d s=\int_{-a}^{a} V\left(x^{\prime}, t\right) \frac{\partial Z}{\partial x^{\prime}}\left(x^{\prime}, t\right) d x^{\prime}=
\end{aligned}
$$




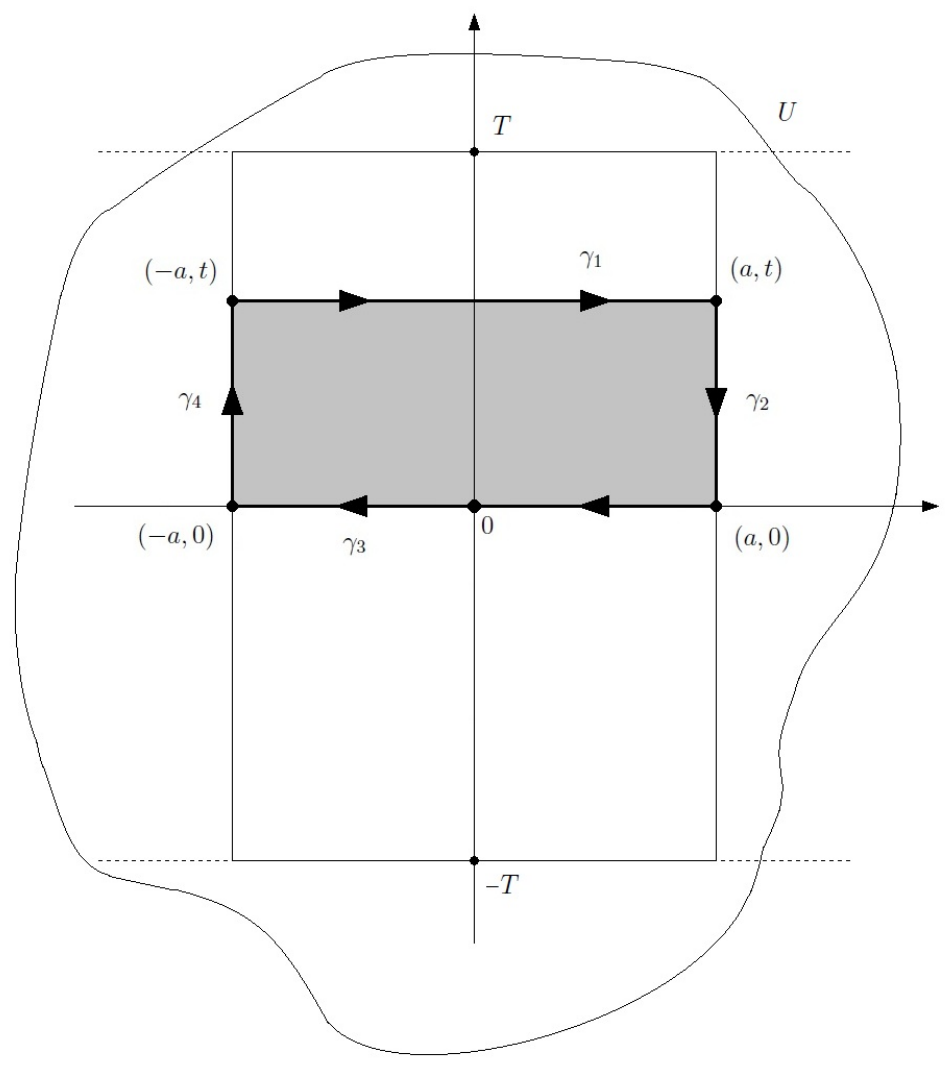

$=\int_{\mathbb{R}} V\left(x^{\prime}, t\right) \frac{\partial Z}{\partial x^{\prime}}\left(x^{\prime}, t\right) d x^{\prime}=G_{\lambda} u(x, t)$.

- $\int_{\gamma_{2}} \omega=\int_{0}^{1}\left[V\left(\gamma_{2}(s)\right) \frac{\partial Z}{\partial x^{\prime}}\left(\gamma_{2}(s)\right)(0)+V\left(\gamma_{2}(s)\right) \frac{\partial Z}{\partial t^{\prime}}\left(\gamma_{2}(s)\right)(-t)\right] d s=$ $=\int_{0}^{1} V(a,-t s+t) \frac{\partial Z}{\partial t^{\prime}}(a,-t s+t)(-t) d s=0$.

- $\int_{\gamma_{3}} \omega=\int_{0}^{1}\left[V\left(\gamma_{3}(s)\right) \frac{\partial Z}{\partial x^{\prime}}\left(\gamma_{3}(s)\right)(-2 a)+V\left(\gamma_{3}(s)\right) \frac{\partial Z}{\partial t^{\prime}}\left(\gamma_{3}(s)\right) 0\right] d s=$ $=\int_{0}^{1} V(-2 a s+a, 0) \frac{\partial Z}{\partial x^{\prime}}(-2 a s+a, 0)(-2 a) d s=\int_{a}^{-a} V\left(x^{\prime}, 0\right) \frac{\partial Z}{\partial x^{\prime}}\left(x^{\prime}, 0\right) d x^{\prime}=$ $=-\int_{-a}^{a} V\left(x^{\prime}, 0\right) \frac{\partial Z}{\partial x^{\prime}}\left(x^{\prime}, 0\right) d x^{\prime}=-\int_{\mathbb{R}} V\left(x^{\prime}, t\right) \frac{\partial Z}{\partial x^{\prime}}\left(x^{\prime}, t\right) d x^{\prime}=-E_{\lambda} u(x, t)$.

- $\int_{\gamma_{4}} \omega=\int_{0}^{1}\left[V\left(\gamma_{4}(s)\right) \frac{\partial Z}{\partial x^{\prime}}\left(\gamma_{4}(s)\right)(0)+V\left(\gamma_{4}(s)\right) \frac{\partial Z}{\partial t^{\prime}}\left(\gamma_{4}(s)\right) t\right] d s=$

$$
=\int_{0}^{1} V(-a, t s) \frac{\partial Z}{\partial t^{\prime}}(-a, t s) t d s=0 .
$$


Daí,

$$
\int_{\gamma_{1} * \gamma_{2} * \gamma_{3} * \gamma_{4}} \omega=\int_{\gamma_{1}} \omega+\int_{\gamma_{2}} \omega+\int_{\gamma_{3}} \omega+\int_{\gamma_{4}} \omega=G_{\lambda} \tilde{u}(x, t)-E_{\lambda} \tilde{u}(x, t) .
$$

Obs. 5.3.7. $G_{\lambda} u(x, 0)=E_{\lambda} u(x, 0)$, para todo $x \in(-a, a)$ e $\lambda>0$. No caso em que $t \neq 0, \gamma_{1} * \gamma_{2} * \gamma_{3} * \gamma_{4}$ é fronteira de $[-a, a] \times[0, t]$, onde $[0, t]$ corresponde ao seguimento que liga 0 a t. Assim podemos aplicar o Teorema de Green e obter

$$
\left|\int_{\gamma_{1} * \gamma_{2} * \gamma_{3} * \gamma_{4}} \omega\right|=\left|\int_{[-a, a] \times[0, t]}\left[\frac{\partial}{\partial x^{\prime}}\left(V\left(x^{\prime}, t^{\prime}\right) \frac{\partial Z}{\partial t^{\prime}}\left(x^{\prime}, t^{\prime}\right)\right)-\frac{\partial}{\partial t^{\prime}}\left(V\left(x^{\prime}, t^{\prime}\right) \frac{\partial Z}{\partial x^{\prime}}\left(x^{\prime}, t^{\prime}\right)\right)\right] d x^{\prime} d t^{\prime}\right| .
$$

Observe que a orientação da curva $\gamma_{1} * \gamma_{2} * \gamma_{3} * \gamma_{4}$ depende do sinal de $t$, i.e., quando $t>0$ a orientação é negativa e quando $t<0$ a orientação é positiva. Devido a isso que a igualdade acima esta expressa em módulo.

Por (5.3.7) e (5.3.8), independente do valor de $t \in(-T, T)$, temos

$$
\begin{gathered}
\left|G_{\lambda} \tilde{u}(x, t)-E_{\lambda} \tilde{u}(x, t)\right|= \\
=\left|\int_{[-a, a] \times[0, t]}\left[\frac{\partial}{\partial x^{\prime}}\left(V\left(x^{\prime}, t^{\prime}\right) \frac{\partial Z}{\partial t^{\prime}}\left(x^{\prime}, t^{\prime}\right)\right)-\frac{\partial}{\partial t^{\prime}}\left(V\left(x^{\prime}, t^{\prime}\right) \frac{\partial Z}{\partial x^{\prime}}\left(x^{\prime}, t^{\prime}\right)\right)\right] d x^{\prime} d t^{\prime}\right| .
\end{gathered}
$$

Desenvolvendo o integrando

$$
\begin{gathered}
{\left[\frac{\partial}{\partial x^{\prime}}\left(V\left(x^{\prime}, t^{\prime}\right) \frac{\partial Z}{\partial t^{\prime}}\left(x^{\prime}, t^{\prime}\right)\right)-\frac{\partial}{\partial t^{\prime}}\left(V\left(x^{\prime}, t^{\prime}\right) \frac{\partial Z}{\partial x^{\prime}}\left(x^{\prime}, t^{\prime}\right)\right)\right]} \\
=\left[\frac{\partial}{\partial x^{\prime}}\left(V\left(x^{\prime}, t^{\prime}\right)\right) \frac{\partial Z}{\partial t^{\prime}}\left(x^{\prime}, t^{\prime}\right)+V\left(x^{\prime}, t^{\prime}\right) \frac{\partial}{\partial x^{\prime}}\left(\frac{\partial Z}{\partial t^{\prime}}\left(x^{\prime}, t^{\prime}\right)\right)\right. \\
\left.-\frac{\partial}{\partial t^{\prime}}\left(V\left(x^{\prime}, t^{\prime}\right)\right) \frac{\partial Z}{\partial x^{\prime}}\left(x^{\prime}, t^{\prime}\right)-V\left(x^{\prime}, t^{\prime}\right) \frac{\partial}{\partial t^{\prime}}\left(\frac{\partial Z}{\partial x^{\prime}}\left(x^{\prime}, t^{\prime}\right)\right)\right] \\
=\frac{\partial}{\partial x^{\prime}}\left(V\left(x^{\prime}, t^{\prime}\right)\right) \frac{\partial Z}{\partial t^{\prime}}\left(x^{\prime}, t^{\prime}\right)-\frac{\partial}{\partial t^{\prime}}\left(V\left(x^{\prime}, t^{\prime}\right)\right) \frac{\partial Z}{\partial x^{\prime}}\left(x^{\prime}, t^{\prime}\right)=\tilde{L}\left(V\left(x^{\prime}, t^{\prime}\right)\right),
\end{gathered}
$$

a menos de um fator $C^{\infty}$ que não se anula. (A inclusão desse fator não altera em nada a estimativa feita abaixo). Por sua vez,

$$
\begin{gathered}
\tilde{L}\left(V\left(x^{\prime}, t^{\prime}\right)\right)=\left(\frac{\lambda}{\pi}\right)^{\frac{1}{2}} \tilde{L}\left(e^{-\lambda\left[Z(x, t)-Z\left(x^{\prime}, t^{\prime}\right)\right]^{2}} \tilde{u}\left(x^{\prime}, t^{\prime}\right) h\left(x^{\prime}\right)\right)= \\
\left(\frac{\lambda}{\pi}\right)^{\frac{1}{2}}\left(\tilde{L}\left(e^{-\lambda\left[Z(x, t)-Z\left(x^{\prime}, t^{\prime}\right)\right]^{2}} h\left(x^{\prime}\right)\right) \tilde{u}\left(x^{\prime}, t^{\prime}\right)+\left(e^{-\lambda\left[Z(x, t)-Z\left(x^{\prime}, t^{\prime}\right)\right]^{2}} h\left(x^{\prime}\right)\right) \tilde{L}\left(\tilde{u}\left(x^{\prime}, t^{\prime}\right)\right)\right)
\end{gathered}
$$




$$
\begin{gathered}
=\left(\frac{\lambda}{\pi}\right)^{\frac{1}{2}} \tilde{L}\left(e^{-\lambda\left[Z(x, t)-Z\left(x^{\prime}, t^{\prime}\right)\right]^{2}} h\left(x^{\prime}\right)\right) \tilde{u}\left(x^{\prime}, t^{\prime}\right) \\
=\left(\frac{\lambda}{\pi}\right)^{\frac{1}{2}}\left(\tilde{L}\left(e^{-\lambda\left[Z(x, t)-Z\left(x^{\prime}, t^{\prime}\right)\right]^{2}}\right) h\left(x^{\prime}\right) \tilde{u}\left(x^{\prime}, t^{\prime}\right)+\left(e^{-\lambda\left[Z(x, t)-Z\left(x^{\prime}, t^{\prime}\right)\right]^{2}}\right) \tilde{L}\left(h\left(x^{\prime}\right)\right) \tilde{u}\left(x^{\prime}, t^{\prime}\right)\right) \\
=\left(\frac{\lambda}{\pi}\right)^{\frac{1}{2}}\left(e^{-\lambda\left[Z(x, t)-Z\left(x^{\prime}, t^{\prime}\right)\right]^{2}} \tilde{L}\left(h\left(x^{\prime}\right)\right) \tilde{u}\left(x^{\prime}, t^{\prime}\right)\right),
\end{gathered}
$$

uma vez que $\tilde{L}\left(e^{-\lambda\left[Z(x, t)-Z\left(x^{\prime}, t^{\prime}\right)\right]^{2}}\right)=0$. De fato, como $e^{z}$ é inteira, basta verificar que $\tilde{L}\left(-\lambda\left[Z(x, t)-Z\left(x^{\prime}, t^{\prime}\right)\right]^{2}\right)=0$. Como $\tilde{L}\left(Z\left(x^{\prime}, t^{\prime}\right)\right)=0$, segue de Leibniz que

$$
\begin{gathered}
\tilde{L}\left(\left[Z(x, t)-Z\left(x^{\prime}, t^{\prime}\right)\right]^{2}\right)=2\left[Z(x, t)-Z\left(x^{\prime}, t^{\prime}\right)\right] \tilde{L}\left(\left[Z(x, t)-Z\left(x^{\prime}, t^{\prime}\right)\right]\right)= \\
=-2\left[Z(x, t)-Z\left(x^{\prime}, t^{\prime}\right)\right] \tilde{L}\left(Z\left(x^{\prime}, t^{\prime}\right)\right)=0 .
\end{gathered}
$$

Portanto, temos que

$$
\begin{gathered}
{\left[\frac{\partial}{\partial x^{\prime}}\left(V\left(x^{\prime}, t^{\prime}\right) \frac{\partial Z}{\partial t^{\prime}}\left(x^{\prime}, t^{\prime}\right)\right)-\frac{\partial}{\partial t^{\prime}}\left(V\left(x^{\prime}, t^{\prime}\right) \frac{\partial Z}{\partial x^{\prime}}\left(x^{\prime}, t^{\prime}\right)\right)\right]} \\
=\left(\frac{\lambda}{\pi}\right)^{\frac{1}{2}}\left(e^{-\lambda\left[Z(x, t)-Z\left(x^{\prime}, t^{\prime}\right)\right]^{2}} \tilde{L}\left(h\left(x^{\prime}\right)\right) \tilde{u}\left(x^{\prime}, t^{\prime}\right)\right) .
\end{gathered}
$$

Assim

$$
\begin{aligned}
\left|G_{\lambda} \tilde{u}(x, t)-E_{\lambda} \tilde{u}(x, t)\right| & =\left(\frac{\lambda}{\pi}\right)^{\frac{1}{2}}\left|\int_{[-a, a] \times[0, t]}\left(e^{-\lambda\left[Z(x, t)-Z\left(x^{\prime}, t^{\prime}\right)\right]^{2}} \tilde{L}\left(h\left(x^{\prime}\right)\right) \tilde{u}\left(x^{\prime}, t^{\prime}\right)\right) d x^{\prime} d t^{\prime}\right| \\
& =\left(\frac{\lambda}{\pi}\right)^{\frac{1}{2}}\left|\int_{\mathbb{R} \times[0, t]}\left(e^{-\lambda\left[Z(x, t)-Z\left(x^{\prime}, t^{\prime}\right)\right]^{2}} \tilde{L}\left(h\left(x^{\prime}\right)\right) \tilde{u}\left(x^{\prime}, t^{\prime}\right)\right) d x^{\prime} d t^{\prime}\right| \\
& \leq\left(\frac{\lambda}{\pi}\right)^{\frac{1}{2}} \int_{\mathbb{R} \times[0, t]}\left|e^{-\lambda\left[Z(x, t)-Z\left(x^{\prime}, t^{\prime}\right)\right]^{2}}\right|\left|\tilde{L}\left(h\left(x^{\prime}\right)\right) \tilde{u}\left(x^{\prime}, t^{\prime}\right)\right| d x^{\prime} d t^{\prime} .
\end{aligned}
$$

Vamos estimar a integral acima. Inicialmente observe que $\tilde{L}(h) \equiv 0$ em $(-a / 2, a / 2) \cup$ $(-\infty,-a) \cup(a, \infty)$, pois $h \equiv 1$ em $(-a / 2, a / 2)$ e $h \equiv 0$ em $(-\infty,-a) \cup(a, \infty)$. Portanto resta estudar o caso em que $x^{\prime} \in(-a,-a / 2) \cup(a / 2, a)$. Seja $(x, t) \in(-a, a) \times(-T, T)$, temos

$$
\left|e^{-\lambda\left[Z(x, t)-Z\left(x^{\prime}, t^{\prime}\right)\right]^{2}}\right|=e^{\Re\left\{-\lambda\left[Z(x, t)-Z\left(x^{\prime}, t^{\prime}\right)\right]^{2}\right\}}=e^{\lambda\left(\left|\phi(x, t)-\phi\left(x^{\prime}, t^{\prime}\right)\right|^{2}-\left|x-x^{\prime}\right|^{2}\right)}
$$


para todo $\left(x^{\prime}, t^{\prime}\right) \in \mathbb{R}^{2}$ e $\lambda>0$. Como $\phi$ tem suporte compacto (e é contínua) existe uma constante $C^{\prime}>0$ tal que $\left|\phi^{\prime}\left(x^{\prime}, t^{\prime}\right)\right| \leq C^{\prime}$, para todo $\left(x^{\prime}, t^{\prime}\right) \in \mathbb{R}^{2}$. Assim pelo teorema da desigualdade do valor médio temos

$$
\left|\phi\left(x^{\prime}, t\right)-\phi\left(x^{\prime}, t^{\prime}\right)\right| \leq C^{\prime}\left|\left(x^{\prime}, t\right)-\left(x^{\prime}, t^{\prime}\right)\right|=C^{\prime}\left|t-t^{\prime}\right|
$$

para todo $\left(x^{\prime}, t^{\prime}\right) \in \mathbb{R}^{2}$. Novamente pelo teorema da desigualdade do valor médio e por $(5.3 .1)$

$$
\left|\phi(x, t)-\phi\left(x^{\prime}, t\right)\right| \leq \frac{1}{2}\left|x-x^{\prime}\right|
$$

para todo $x^{\prime} \in(-a, a)$. Assim,

$$
\begin{aligned}
\left|\phi(x, t)-\phi\left(x^{\prime}, t^{\prime}\right)\right| & \leq\left|\phi(x, t)-\phi\left(x^{\prime}, t\right)\right|+\left|\phi\left(x^{\prime}, t\right)-\phi\left(x^{\prime}, t^{\prime}\right)\right| \\
& \leq \frac{1}{2}\left|x-x^{\prime}\right|+C^{\prime}\left|t-t^{\prime}\right|
\end{aligned}
$$

para $\left(x^{\prime}, t^{\prime}\right) \in(-a, a) \times(-T, T)$. Como $t^{\prime} \in[0, t]$ e $|t| \leq T$ temos $\left|t-t^{\prime}\right| \leq T$. De fato, se $t \neq 0$, então

$$
\left|t-t^{\prime}\right|=\left|t-\left(\left|t^{\prime}\right| /|t|\right) t\right|=\left|1-\left(\left|t^{\prime}\right| /|t|\right)\right||t| \leq|t| \leq T
$$

Portanto

$$
\left|\phi(x, t)-\phi\left(x^{\prime}, t^{\prime}\right)\right| \leq \frac{1}{2}\left|x-x^{\prime}\right|+C^{\prime} T
$$

para todo $x^{\prime} \in(-a, a)$ e $t^{\prime} \in(-t, t)$. Observe que tomando $\left|x^{\prime}\right| \geq a / 2$ temos

$$
\left|x-x^{\prime}\right| \geq\left|x^{\prime}\right|-|x| \geq \frac{a}{2}-\frac{a}{4}=\frac{a}{4},
$$


para $|x|<a / 4$. Daí,

$$
\begin{aligned}
\left|\phi(x, t)-\phi\left(x^{\prime}, t^{\prime}\right)\right|^{2}-\left|x-x^{\prime}\right|^{2} & \leq\left(\frac{1}{2}\left|x-x^{\prime}\right|+C^{\prime} T\right)^{2}-\left|x-x^{\prime}\right|^{2} \\
& \leq 2\left(\frac{1}{4}\left|x-x^{\prime}\right|^{2}+\left(C^{\prime} T\right)^{2}\right)-\left|x-x^{\prime}\right|^{2} \\
& =\frac{1}{2}\left|x-x^{\prime}\right|^{2}+2\left(C^{\prime} T\right)^{2}-\left|x-x^{\prime}\right|^{2} \\
& =-\frac{1}{2}\left|x-x^{\prime}\right|^{2}+2\left(C^{\prime} T\right)^{2} \\
& \leq-\frac{a^{2}}{32}+2\left(C^{\prime} T\right)^{2}
\end{aligned}
$$

para $(x, t) \in(-a / 2, a / 2) \times(-T, T), x^{\prime} \in(-a,-a / 2) \cup(a / 2, a)\left(\right.$ e $\left.t^{\prime} \in(-t, t)\right)$.

Fazendo $T<\frac{a}{8 C^{\prime}}$, temos que $\delta \doteq-\frac{a^{2}}{32}+2\left(C^{\prime} T\right)^{2}<0$.

Assim, para $(x, t) \in(-a / 4, a / 4) \times(-T, T)$ temos

$$
\begin{aligned}
\left|G_{\lambda} \tilde{u}(x, t)-E_{\lambda} \tilde{u}(x, t)\right| & \leq\left(\frac{\lambda}{\pi}\right)^{\frac{1}{2}} \int_{\mathbb{R} \times[0, t]}\left|e^{-\lambda\left[Z(x, t)-Z\left(x^{\prime}, t^{\prime}\right)\right]^{2}}\right|\left|\tilde{L}\left(h\left(x^{\prime}\right)\right) \tilde{u}\left(x^{\prime}, t^{\prime}\right)\right| d x^{\prime} d t^{\prime} \\
& \leq\left(\frac{\lambda}{\pi}\right)^{\frac{1}{2}} e^{\lambda \delta} \int_{\mathbb{R} \times[0, t]}\left|\tilde{L}\left(h\left(x^{\prime}\right)\right) \tilde{u}\left(x^{\prime}, t^{\prime}\right)\right| d x^{\prime} d t^{\prime} \leq N\left(\frac{\lambda}{\pi}\right)^{\frac{1}{2}} e^{\lambda \delta},
\end{aligned}
$$

para todo $\lambda>0$. Portanto

$$
\left|G_{\lambda} \tilde{u}-E_{\lambda} \tilde{u}\right| \longrightarrow 0
$$

uniformemente em $(-a / 4, a / 4) \times(-T, T)$ quando $\lambda \longrightarrow \infty$. Daí concluímos que

$$
E_{\lambda} \tilde{u} \longrightarrow \tilde{u}
$$

uniformemente em $(-a / 4, a / 4) \times(-T, T)$ quando $\lambda \longrightarrow \infty$.

Para começar a ultima etapa da demonstração, para cada $\lambda>0$ fixado, seja 


$$
H_{\lambda}(x, y) \doteq\left(\frac{\lambda}{\pi}\right)^{\frac{1}{2}} \int_{\mathbb{R}} e^{-\lambda\left[(x+i y)-Z\left(x^{\prime}, 0\right)\right]^{2}} \tilde{u}\left(x^{\prime}, 0\right) h\left(x^{\prime}\right) \frac{\partial Z}{\partial x}\left(x^{\prime}, 0\right) d x^{\prime}
$$

para $(x, y) \in \mathbb{R}^{2}$.

Seja

$$
f_{\lambda}\left(x, y, x^{\prime}\right)=e^{-\lambda\left[(x+i y)-Z\left(x^{\prime}, 0\right)\right]^{2}} \tilde{u}\left(x^{\prime}, 0\right) h\left(x^{\prime}\right) \frac{\partial Z}{\partial x}\left(x^{\prime}, 0\right),
$$

para $\left(x, y, x^{\prime}\right) \in \mathbb{R}^{3}$.

Seja $\left(x_{0}, y_{0}\right) \in \mathbb{R}^{2}$ e $\epsilon>0$. Como $f_{\lambda}\left(x, y, x^{\prime}\right)=0$, para $x^{\prime} \in \mathbb{R}$ com $\left|x^{\prime}\right|>0$, podemos supor que $x^{\prime} \in[-a, a]$. Temos

$$
\begin{aligned}
& \left|\frac{H_{\lambda}\left(x_{0}+s, y_{0}\right)-H_{\lambda}\left(x_{0}, y_{0}\right)}{s}-\left(\frac{\lambda}{\pi}\right)^{\frac{1}{2}} \int_{\mathbb{R}} \frac{\partial f_{\lambda}}{\partial x}\left(x_{0}, y_{0}, x^{\prime}\right) d x^{\prime}\right|= \\
& =\left|\frac{1}{s}\left(\frac{\lambda}{\pi}\right)^{\frac{1}{2}} \int_{\mathbb{R}}\left(f_{\lambda}\left(x_{0}+s, y_{0}, x^{\prime}\right)-f_{\lambda}\left(x_{0}, y_{0}, x^{\prime}\right)-\frac{\partial f_{\lambda}}{\partial x}\left(x_{0}, y_{0}, x^{\prime}\right) s\right) d x^{\prime}\right| \\
& =\left|\frac{1}{s}\left(\frac{\lambda}{\pi}\right)^{\frac{1}{2}} \int_{-a}^{a}\left(f_{\lambda}\left(x_{0}+s, y_{0}, x^{\prime}\right)-f_{\lambda}\left(x_{0}, y_{0}, x^{\prime}\right)-\frac{\partial f_{\lambda}}{\partial x}\left(x_{0}, y_{0}, x^{\prime}\right) s\right) d x^{\prime}\right| \\
& \leq \frac{1}{|s|}\left(\frac{\lambda}{\pi}\right)^{\frac{1}{2}} \int_{-a}^{a}\left|f_{\lambda}\left(x_{0}+s, y_{0}, x^{\prime}\right)-f_{\lambda}\left(x_{0}, y_{0}, x^{\prime}\right)-\frac{\partial f_{\lambda}}{\partial x}\left(x_{0}, y_{0}, x^{\prime}\right) s\right| d x^{\prime} .
\end{aligned}
$$

Por outro lado, temos

$$
\begin{aligned}
& f_{\lambda}\left(x_{0}+s, y_{0}, x^{\prime}\right)- f_{\lambda}\left(x_{0}, y_{0}, x^{\prime}\right)-\frac{\partial f_{\lambda}}{\partial x}\left(x_{0}, y_{0}, x^{\prime}\right) s= \\
&=\int_{0}^{1}\left[\frac{\partial f_{\lambda}}{\partial x}\left(x_{0}+r s, y_{0}, x^{\prime}\right)-\frac{\partial}{\partial x}\left(f_{\lambda}\left(x_{0}, y_{0}, x^{\prime}\right)\right)\right] d r
\end{aligned}
$$

para $x^{\prime} \in[-a, a]$. Daí,

$$
\begin{aligned}
& \mid f_{\lambda}\left(x_{0}+s, y_{0}, x^{\prime}\right)- f_{\lambda}\left(x_{0}, y_{0}, x^{\prime}\right)-\frac{\partial f_{\lambda}}{\partial x}\left(x_{0}, y_{0}, x^{\prime}\right) s \mid \leq \\
& \leq|s| \sup _{0 \leq r \leq 1}\left|\frac{\partial f_{\lambda}}{\partial x}\left(x_{0}+r s, y_{0}, x^{\prime}\right)-\frac{\partial f_{\lambda}}{\partial x}\left(x_{0}, y_{0}, x^{\prime}\right)\right|
\end{aligned}
$$

para $x^{\prime} \in[-a, a]$. Como $\frac{\partial f_{\lambda}}{\partial x}\left(x, y, x^{\prime}\right) \in C^{\infty}\left(\mathbb{R}^{3}\right)$ e $[-a, a]$ é compacto, segue que a aplicação 


$$
\mathbb{R} \times[-a, a] \ni\left(x, x^{\prime}\right) \mapsto \frac{\partial f_{\lambda}}{\partial x}\left(x, y_{0}, x^{\prime}\right)
$$

é contínua em $x$ de modo uniforme em relação a $x^{\prime}$. Portanto, existe $\delta>0$ tal que $|s|<\delta$ acarreta

$$
\left|\frac{\partial f_{\lambda}}{\partial x}\left(x_{0}+r s, y_{0}, x^{\prime}\right)-\frac{\partial f_{\lambda}}{\partial x}\left(x_{0}, y_{0}, x^{\prime}\right)\right|<\frac{1}{2 a}\left(\frac{\lambda}{\pi}\right)^{-\frac{1}{2}} \epsilon
$$

para todo $x^{\prime} \in[-a, a]$. Logo, para $|s|<\delta$, temos

$$
\left|f_{\lambda}\left(x_{0}+s, y_{0}, x^{\prime}\right)-f_{\lambda}\left(x_{0}, y_{0}, x^{\prime}\right)-\frac{\partial f_{\lambda}}{\partial x}\left(x_{0}, y_{0}, x^{\prime}\right) s\right| \leq|s| \epsilon
$$

para todo $x^{\prime} \in[-a, a]$. Daí,

$$
\left|\frac{H_{\lambda}\left(x_{0}+s, y_{0}\right)-H_{\lambda}\left(x_{0}, y_{0}\right)}{s}-\left(\frac{\lambda}{\pi}\right)^{\frac{1}{2}} \int_{\mathbb{R}} \frac{\partial f_{\lambda}}{\partial x}\left(x_{0}, y_{0}, x^{\prime}\right) d x^{\prime}\right| \leq \epsilon
$$

Assim,

$$
\frac{\partial H_{\lambda}}{\partial x}\left(x_{0}, y_{0}\right)=\left(\frac{\lambda}{\pi}\right)^{\frac{1}{2}} \int_{\mathbb{R}} \frac{\partial f_{\lambda}}{\partial x}\left(x_{0}, y_{0}, x^{\prime}\right) d x^{\prime}
$$

Pela arbitrariedade de $\left(x_{0}, y_{0}\right) \in \mathbb{R}^{2}$, temos

$$
\frac{\partial H_{\lambda}}{\partial x}(x, y)=\left(\frac{\lambda}{\pi}\right)^{\frac{1}{2}} \int_{\mathbb{R}} \frac{\partial f_{\lambda}}{\partial x}\left(x, y, x^{\prime}\right) d x^{\prime}
$$

para todo $(x, y) \in \mathbb{R}^{2}$.

De forma análoga, podemos mostrar que

$$
\frac{\partial H_{\lambda}}{\partial y}(x, y)=\left(\frac{\lambda}{\pi}\right)^{\frac{1}{2}} \int_{\mathbb{R}} \frac{\partial f_{\lambda}}{\partial y}\left(x, y, x^{\prime}\right) d x^{\prime}
$$

para todo $(x, y) \in \mathbb{R}^{2}$.

Assim,

$$
\begin{aligned}
\frac{\partial}{\partial \bar{z}}\left(H_{\lambda}\right)(x, y) & =\frac{\partial H_{\lambda}}{\partial x}(x, y)+i \frac{\partial H_{\lambda}}{\partial y}(x, y) \\
& =\left(\frac{\lambda}{\pi}\right)^{\frac{1}{2}} \int_{\mathbb{R}} \frac{\partial f_{\lambda}}{\partial x}\left(x, y, x^{\prime}\right) d x^{\prime}+i\left(\frac{\lambda}{\pi}\right)^{\frac{1}{2}} \int_{\mathbb{R}} \frac{\partial f_{\lambda}}{\partial y}\left(x, y, x^{\prime}\right) d x^{\prime} \\
& =\left(\frac{\lambda}{\pi}\right)^{\frac{1}{2}} \int_{\mathbb{R}} \frac{\partial f_{\lambda}}{\partial x}\left(x, y, x^{\prime}\right)+i \frac{\partial f_{\lambda}}{\partial y}\left(x, y, x^{\prime}\right) d x^{\prime} \\
& =\left(\frac{\lambda}{\pi}\right)^{\frac{1}{2}} \int_{\mathbb{R}} \frac{\partial f_{\lambda}}{\partial \bar{z}}\left(x, y, x^{\prime}\right) d x^{\prime}
\end{aligned}
$$


para todo $(x, y) \in \mathbb{R}^{2}$. Ou seja,

$$
\frac{\partial}{\partial \bar{z}}\left(H_{\lambda}\right)(x, y)=\left(\frac{\lambda}{\pi}\right)^{\frac{1}{2}} \int_{\mathbb{R}} \frac{\partial}{\partial \bar{z}}\left(e^{-\lambda\left[(x+i y)-Z\left(x^{\prime}, 0\right)\right]^{2}}\right) \tilde{u}\left(x^{\prime}, 0\right) h\left(x^{\prime}\right) \frac{\partial Z}{\partial x}\left(x^{\prime}, 0\right) d x^{\prime}
$$

para todo $(x, y) \in \mathbb{R}^{2}$. Agora observe que

$$
\frac{\partial}{\partial \bar{z}}\left(e^{-\lambda\left[(x+i y)-Z\left(x^{\prime}, 0\right)\right]^{2}}\right)=-2 \lambda e^{-\lambda\left[(x+i y)-Z\left(x^{\prime}, 0\right)\right]^{2}} \frac{\partial}{\partial \bar{z}}\left((x+i y)-Z\left(x^{\prime}, 0\right)\right)=0,
$$

para todo $(x, y) \in \mathbb{R}^{2}$. Assim temos $\frac{\partial}{\partial \bar{z}}\left(H_{\lambda}\right)(x, y)=0$ para todo $(x, y) \in \mathbb{R}^{2}$. Portanto

$$
H_{\lambda}(z) \doteq\left(\frac{\lambda}{\pi}\right)^{\frac{1}{2}} \int_{\mathbb{R}} e^{-\lambda\left[(x+i y)-Z\left(x^{\prime}, 0\right)\right]^{2}} \tilde{u}\left(x^{\prime}, 0\right) h\left(x^{\prime}\right) \frac{\partial Z}{\partial x}\left(x^{\prime}, 0\right) d x^{\prime},
$$

com $z=x+i y$ é analítica em $\mathbb{C}$, em outras palavras o raio de convergência da sua série de potências centrada na origem é infinito. Em particular sua série de potência converge uniformemente em todo disco (fechado) $\bar{D}_{r}(0)$ centrado na origem de raio $r>0$. Logo $\left(H_{j}\right)_{j \in \mathbb{N}}$ é uma sequência de funções inteiras.

Seja dado $\epsilon>0$. Escolha $r$ de modo que $Z((-a / 4, a / 4) \times(-T, T)) \subset \bar{D}_{r}(0)$. Pelo cometário acima, podemos obter para cada $j \in \mathbb{N}$ um polinômio $P_{j}$ tal que $\mid H_{j}(z)-$ $P_{j}(z) \mid<1 / j$, para todo $z \in \bar{D}_{r}(0)$. Daí, $\left|H_{j}(Z(x, t))-P_{j}(Z(x, t))\right|<\epsilon / 2$, para todo $(x, t) \in(-a / 4, a / 4) \times(-T, T)$. Por outro lado, pelo que foi mostrado acima, temos que existe $j_{0} \in \mathbb{N}, j_{0}>2 / \epsilon$ tal que $j \in \mathbb{N}$, com $j \geq j_{0}$ implica $\left|\tilde{u}(x, t)-E_{j} \tilde{u}(x, t)\right|<\epsilon / 2$, para todo $(x, t) \in(-a / 4, a / 4) \times(-T, T)$. Portanto para $j \geq j_{0}$,

$$
\begin{gathered}
\left|\tilde{u}(x, t)-P_{j}(Z(x, t))\right| \leq\left|\tilde{u}(x, t)-E_{j} \tilde{u}(x, t)\right|+\left|E_{j}(Z(x, t))-P_{j}(Z(x, t))\right| \\
<\frac{\epsilon}{2}+\frac{\epsilon}{2}=\epsilon
\end{gathered}
$$

para todo $(x, t) \in(-a / 4, a / 4) \times(-T, T)$. Ou seja, $P_{j} \circ Z \longrightarrow \tilde{u}$ uniformemente em $(-a / 4, a / 4) \times(-T, T)$. E assim a segunda etapa da demonstração esta completa.

Segue da segunda etapa que

$$
P_{j}(Z \circ \Phi) \longrightarrow(\tilde{u} \circ \Phi)
$$

uniformemente em $W \doteq \Phi^{-1}((-a / 4, a / 4) \times(-T, T)) \subset V \subset U$ quando $j \longrightarrow \infty$. Ou seja, 


$$
\left(P_{j} \circ Q\right)\left(Z_{U}\right) \longrightarrow u
$$

uniformemente em $W \subset U$ quando $j \longrightarrow \infty$.

Observe que $P_{j} \circ Q$ é um polinômio para todo $j \in \mathbb{N}$.

\subsubsection{Estrutura Local das Soluções}

Seja $L$ um campo vetorial complexo não singular e localmente integrável definido em $\Omega$, onde $\Omega$ é um aberto conexo de $\mathbb{R}^{2}$.

Agora vamos usar o teorema de Baouendi-Treves para escrever qualquer solução da homogênea em função de uma integral primeira do campo. Sabemos que se $Z$ é solução da homogênea e $C^{\infty}$, e $h$ é uma função holomorfa cujo domínio contém a imagem de $Z$, então $h \circ Z$ também é solução da homogênea. Assim, surge a seguinte pergunta:

(**) fixado um ponto $p$ no dominio de $L$ e dadas duas soluções da homogênea u e $Z$ funções $C^{\infty}$ em uma vizinhança de $p$, existe uma função holomorfa $h$, tal que $u=h \circ Z$ em uma vizinhança de $p$ ?

Uma observação simples, porém importante na busca de condições necessárias para responder à pergunta acima, é relacionar as imagens das duas soluções. Por exemplo quando uma das soluções é constante, e.g., $Z$ é uma aplicação constante (funções constantes são sempre soluções de um campo) e $u$ é uma solução que não é constante. Neste caso é fácil ver que não existe $h$ holomorfa, tal que $u=h \circ Z$. Outro exemplo, é quando a imagem de $u$ tem interior não vazio. Neste caso uma condição necessária é que a imagem de $Z$ tenha interior não vazio. Por esta observação, é razoável pensar que $Z$ ser uma solução da homogênea com $d Z \neq 0$ e com imagem "regular", (digamos $Z$ uma aplicação aberta) é uma boa condição para responder a pergunta acima. O próximo teorema diz que esta condição é suficiente. 
Teorema 5.3.8. Suponha $\mathcal{U}$ uma cobertura aberta de $\Omega$ e seja $\left\{\left(U, Z_{U}\right)\right\}_{U \in \mathcal{U}}$ uma familia de pares associada à cobertura $\mathcal{U}$ tal que para cada $U \in \mathcal{U}, Z_{U}: U \longrightarrow \mathbb{C}$ é uma aplicação aberta de classe $C^{\infty}$ que satisfaz $L Z_{U}=0$ e $d Z_{U} \neq 0$ em $U$. Seja $p_{0} \in \Omega$ e seja $u \in C^{\infty}(\Omega)$ tal que Lu $=0 \mathrm{em} V$, onde $V$ é uma vizinhança aberta de $p_{0}$ em $\Omega$. Então, existe $W$ uma vizinhança aberta de $p_{0}$ tal que $W \subset U \cap V$, para algum $U \in \mathcal{U}$ e existe uma função holomorfa $h: Z_{U}(W) \longrightarrow \mathbb{C}$ tal que

$$
u=h \circ Z_{U} e m W
$$

\section{Demonstração:}

Seja $U \in \mathcal{U}$ tal que $p_{0} \in U$ e seja $W \subset U$ a vizinhança aberta de $p_{0}$ dada pelo teorema de Baouendi-Treves. Reduzindo $W$ se necessário, podemos supor que $W \subset V \cap U$. Considere $\left.Z \doteq Z_{U}\right|_{W}$. Note que o fato de $Z_{U}: U \longrightarrow \mathbb{C}$ ser uma aplicação aberta é essencial nesta demonstração, uma vez que $Z(W)$ deve ser um aberto de $\mathbb{C}$ para que a tese do teorema tenha sentido. Considere a seguinte relação de equivalência em $W$ :

$$
p \sim q \Longleftrightarrow Z(p)=Z(q)
$$

Seja $[p]$ a classe de equivalência do ponto $p \in W$ e $\mathbb{W}$ o conjunto das classes de equivalência.

Pelo teorema de Baouendi-Treves existe uma sequência de polinômios $\left(P_{j}\right)_{j \in \mathbb{N}}$ tal que $P_{j}(Z) \longrightarrow u$ uniformemente em $W$. Em particular, fica bem definida a seguinte função:

$$
\begin{aligned}
\tilde{u}: \mathbb{W} & \longrightarrow \mathbb{C} \\
{[p] } & \longmapsto u(p)
\end{aligned}
$$

De fato, se $Z(p)=Z(q)$ então $P_{j}(Z(p))=P_{j}(Z(q))$, para todo $j \in \mathbb{N}$, logo, $u(p)=u(q)$.

Definimos

$$
h(w)=\tilde{u}\left(\left[Z^{-1}(w)\right]\right), \text { para } w \in Z(W) .
$$

A função $h$ está bem definida; mais ainda, $h$ é holomorfa. De fato, seja dado $\epsilon>0$. Temos que existe $N_{0} \in \mathbb{N}$, tal que 


$$
j \geq N_{0} \Longrightarrow\left|P_{j}(Z(p))-u(p)\right|<\epsilon, \forall p \in W
$$

Assim, dado $w \in Z(W)$, seja $p \in\left[Z^{-1}(w)\right]$. Logo $P_{j}(w)=P_{j}(Z(p))$ e $h(w)=u(p)$. Logo,

$$
\left|P_{j}(w)-h(w)\right|<\epsilon \text {, para } j \geq N_{0} \text {. }
$$

Pela arbitrariedade de $w \in Z(W)$, temos que $P_{j} \longrightarrow h$ uniformemente em $Z(W)$. Segue do teorema (1.4.11) que $h$ é holomorfa em $Z(W)$.

Apesar de que o teorema acima não menciona a palavra hipoelítico, este conceito está presente, como se pode ver na seguinte proposição:

Proposição 5.3.9. Seja L um campo não singular que possui uma familia $\left\{\left(U, Z_{U}\right)\right\}_{U \in \mathcal{U}}$, onde $\mathcal{U}$ é uma cobertura aberta de $\Omega$ tal que, para cada $U \in \mathcal{U}, Z_{U}: U \longrightarrow \mathbb{C}$ é uma aplicação aberta de classe $C^{\infty}$ que satisfaz $L Z_{U}=0$ e $d Z_{U} \neq 0$ em $U$. Então $L$ é um campo hipoelítico.

\section{Demonstração:}

Seja $p_{0} \in \Omega$. Como $\mathcal{U}$ é uma cobertura de $\Omega$, existe $U \in \mathcal{U}$ tal que $p_{0} \in U$. Associado a $U$, existe $Z_{U}: U \longrightarrow \mathbb{C}$ aberta e com $d Z \neq 0$ em $U$. Pelo lema 1.5.28 existem um polinômio $Q$ de grau 1 e difeomorfismo $\Phi: U^{\prime} \longrightarrow R_{\epsilon \epsilon}(0)$, onde $U^{\prime} \subset U$ é um aberto que contém $p_{0}$, tais que $Z \doteq\left(\Phi^{-1}\right)^{*}\left(Q \circ\left(\left.Z_{U}\right|_{U^{\prime}}\right)\right)(x, t)=x+i \phi(x, t),(x, t) \in R_{\epsilon \epsilon}(0)$, com $\phi$ função a valores reais. Segue do corolário 5.2.12 que $Z$ é injetiva. Daí, concluímos que $\left.Z_{U}\right|_{U^{\prime}}$ é injetiva. Pela arbitrariedade de $p_{0} \in \Omega$, obtemos uma família de pares que satisfaz a condição 2 do teorema 5.2.1. Logo $L$ é hipoelítico em $\Omega$.

Assim, a partir de agora, vamos supor que $L$ é um campo vetorial complexo não singular e hipoelítico. Associada a $L$ vamos considerar a família $\mathcal{A}=\left\{\left(U_{\alpha}, Z_{\alpha}\right)\right\}_{\alpha \in J}$ dada pelo teorema 5.2.1. Pela observação 5.2.13 temos que para cada $\alpha \in J$ a função $Z_{\alpha}: U_{\alpha} \longrightarrow \mathbb{C}$ é uma aplicação aberta. Assim, $\mathcal{A}$ tem as propriedades da família $\mathcal{U}$, com o acréscimo de que dado um par $\left(U_{\alpha}, Z_{\alpha}\right) \in \mathcal{A}$, a função $Z_{\alpha}: U_{\alpha} \longrightarrow \mathbb{C}$ é injetiva. Essa propriedade extra é a chave do resultado dado pelo próximo teorema. 
Teorema 5.3.10. Seja $p_{0} \in \Omega$ e u $\in \mathscr{D}^{\prime}(\Omega)$ tal que Lu $=0$ em $V$, onde $V$ é uma vizinhança aberta de $p_{0}$ em $\Omega$. Então existe uma função holomorfa $h: Z_{\alpha}\left(V \cap U_{\alpha}\right) \longrightarrow \mathbb{C}$, tal que

$$
u=h \circ Z_{\alpha} e m V \cap U_{\alpha}
$$

onde $\left(U_{\alpha}, Z_{\alpha}\right) \in \mathcal{A} \operatorname{com} p_{0} \in U_{\alpha}$.

\section{Demonstração:}

Inicialmente, note que $u \in C^{\infty}(V)$, pois $L u=0$ em $V$ e $L$ é hipoelítico. Como $\left\{U_{\alpha}\right\}_{\alpha \in J}$ é uma cobertura de $\Omega$, existe $\alpha \in J$ tal que $p_{o} \in U_{\alpha}$. Temos que $Z_{\alpha}: U_{\alpha} \longrightarrow \mathbb{C}$ é injetiva. Daí, fica bem definida a função $Z_{\alpha}^{-1}: Z_{\alpha}\left(U_{\alpha}\right) \longrightarrow U_{\alpha}$ e é contínua, pois $Z_{\alpha}$ é aberta. Assim, definimos

$$
h=u \circ Z_{\alpha}^{-1} \text { em } Z_{\alpha}\left(V \cap U_{\alpha}\right) .
$$

Seja $p \in V \cap U_{\alpha}$. Pelo teorema de Baouendi-Treves, existe um aberto $W_{p}$ de $\Omega$ tal que $p \in W_{p} \subset \overline{W_{p}} \subset V \cap U_{\alpha}$ e existe uma sequência de polinômios $\left(P_{j}\right)_{j \in \mathbb{N}}$, tal que $u$ é limite uniforme da sequência $P_{j}\left(Z_{\alpha}\right)$ em $W_{p}$. Vamos estudar a restrição de $h$ a $Z_{\alpha}\left(W_{p}\right)$. Note que a hipoeliticidade é essencial nessa demonstração, uma vez que, via o teorema 5.2.1, $Z_{\alpha}$ é uma aplicação aberta, e em particular, $Z_{\alpha}\left(W_{p}\right)$ é um aberto. Temos que existe $N_{0} \in \mathbb{N}$, tal que

$$
j \geq N_{0} \Longrightarrow\left|P_{j}\left(Z_{\alpha}(q)\right)-u(q)\right|<\epsilon, \forall q \in W_{p}
$$

Assim, dado $w \in Z_{\alpha}\left(W_{p}\right)$, seja $q=Z^{-1}(w)$. Logo $P_{j}(w)=P_{j}(Z(q))$ e $h(w)=u(q)$. Logo,

$$
\left|P_{j}(w)-h(w)\right|<\epsilon \text {, para } j \geq N_{0} .
$$

Pela arbitrariedade de $w \in Z_{\alpha}\left(W_{p}\right)$, temos que $P_{j} \longrightarrow h$ uniformemente em $Z_{\alpha}\left(W_{p}\right)$. Pelo teorema 1.4.11 temos que $h$ é holomorfa em $Z_{\alpha}\left(W_{p}\right)$. Por fim, observe que repetindo o processo para cada $p \in V \cap U_{\alpha}$, obtemos $\left\{W_{p}\right\}_{p \in V \cap U_{\alpha}}$ uma cobertura aberta de $V \cap U_{\alpha}$. Daí, $\left\{Z_{\alpha}\left(W_{p}\right)\right\}_{p \in V \cap U_{\alpha}}$ é uma cobertura aberta de $Z_{\alpha}\left(V \cap U_{\alpha}\right)$. Como mostramos acima $\left.h\right|_{Z_{\alpha}\left(W_{p}\right)}$ é holomorfa, para todo $p \in V \cap U_{\alpha}$. Portanto, $h$ é holomorfa em $Z_{\alpha}\left(V \cap U_{\alpha}\right)$, e vale $u=h \circ Z_{\alpha}$ em $V \cap U_{\alpha}$. 
A grande diferença entre os teoremas 5.3.8 e 5.3.10 é o aberto onde a solução $u$ é igual a uma integral primeira do campo composta com uma função holomorfa. No teorema 5.3.8 obtemos um aberto contido na interseção dos domínios de $u$ e de uma integral primeira do campo, onde $u$ é igual a uma integral primeira do campo composta com uma função holomorfa. Já no teorema 5.3.10 o aberto onde $u$ é igual a uma integral primeira do campo composta com uma função holomorfa é exatamente a interseção dos domínios de $u$ e da uma integral primeira do campo. A princípio essa diferença não parece ter muita importância, uma vez que a maioria resultados envolvendo soluções é de caráter local. No entanto, essa diferença, ou melhor dizendo, a propriedade extra dada pelo teorema 5.3.10, será crucial nos argumentos da próxima seção. Mais precisamente, na construção de um atlas em $\Omega$ a partir de um campo vetorial complexo não singular hipoelítico definido em $\Omega$.

Do teorema 5.3.10 segue um caracterização interessante de soluções de um campo hipoelítico, dada pelo carolário abaixo.

Corolário 5.3.11. Seja L um campo vetorial complexo não singular hipoelítico definido no aberto conexo $\Omega$ de $\mathbb{R}^{2}$. Suponha que $u \in \mathscr{D}^{\prime}(U), U \subset \Omega$ aberto, tal que Lu $=0$ em $U$. Então, $u \in C^{\infty}(U)$ é uma aplicação aberta ou é constante.

\section{Demonstração:}

Como $L$ é hipoelítico em $U$ e $L u=0$ em $U$, então $u \in C^{\infty}(U)$. Pelo teorema 5.3.10 para cada $p \in U$, podemos escrever

$$
u=h_{U_{p}} \circ Z_{U_{p}} \text { em } U_{p}
$$

onde $Z_{\alpha}: U_{\alpha} \longrightarrow \mathbb{C}$ é uma aplicação aberta, onde $U_{p}$ é uma vizinhança aberta de $p$ em $U, \operatorname{com} L Z_{U_{p}}=0$ em $U_{p}$ e $h: Z_{U_{p}}\left(U_{p}\right) \longrightarrow \mathbb{C}$ uma função holomorfa.

Suponha que $u$ não é constante. Logo, $h_{U_{p}}$ não é constante para todo $p \in U$, e como $h_{U_{p}}$ é holomorfa temos que $h_{U_{p}}$ é aberta para todo $p \in U$. Assim, $u$ é uma composição de aplicações abertas em $U_{p}$ para todo $p \in U$. Ou seja, $\left.u\right|_{U_{p}}$ é aberta para todo $p \in U$. Seja $V \subset U$ um aberto. Temos que $V$ pode ser escrito como 


$$
V=\bigcup_{p \in U} V \cap U_{p}
$$

Daí,

$$
u(V)=\bigcup_{p \in U} u\left(V \cap U_{p}\right)
$$

Como $\left.u\right|_{U_{p}}$ é aberta para todo $p \in U$, temos $u\left(V \cap U_{p}\right)$ é um aberto para todo $p \in U$. Da expressão acima temos que $u(V)$ é um aberto de $\mathbb{C}$. Pela arbitrariedade de $V \subset U$ aberto, segue que $u$ é aberta.

No caso em que $L$ é o operador de Cauchy-Riemann a conclusão do corolário acima é natural, i.e., as soluções do operador de Cauchy-Riemann são funções holomorfas que possuem a propriedade de ser aberta ou constante.

Obs. 5.3.12. Seja $M$ um campo definido em $\Omega$. Suponha que $\mathcal{V}$ uma cobertura aberta de $\Omega$ e $\left\{\left(V, Z_{V}\right)\right\}_{V \in \mathcal{V}}$ uma familia de pares associada a cobertura $\mathcal{V}$, tal que para cada $V \in \mathcal{V}$, $Z_{V}: V \longrightarrow \mathbb{C}$ é uma aplicação aberta de classe $C^{\infty}$ que satisfaz $M Z_{V}=0$ em $V$. Não podemos concluir que $M$ é hipoelítico. De fato, basta observar o exemplo em que

$$
M=x^{2} \frac{\partial}{\partial t}+i t^{2} \frac{\partial}{\partial x}
$$

definido em $\mathbb{R}^{2}$. A função $Z(x, t)=x^{3}-i t^{3},(x, t) \in \mathbb{R}^{2}$, é uma aplicação aberta e satisfaz $M Z=0$ em $\mathbb{R}^{2}$. No entanto, $M$ não é hipoelítico. De fato, seja $u=\delta \in \mathscr{D}^{\prime}\left(\mathbb{R}^{2}\right.$ ) (delta de Dirac), dado $\phi \in C_{c}^{\infty}\left(\mathbb{R}^{2}\right)$, temos

$$
\begin{aligned}
\langle M u, \phi\rangle & =\left\langle x^{2} \frac{\partial u}{\partial t}+i t^{2} \frac{\partial u}{\partial x}, \phi\right\rangle=\left\langle x^{2} \frac{\partial u}{\partial t}, \phi\right\rangle+i\left\langle t^{2} \frac{\partial u}{\partial x}, \phi\right\rangle \\
& =\left\langle u,-\frac{\partial\left(x^{2} \phi\right)}{\partial t}\right\rangle+i\left\langle u,-\frac{\partial\left(t^{2} \phi\right)}{\partial x}\right\rangle=\left\langle\delta,-x^{2} \frac{\partial \phi}{\partial t}\right\rangle+i\left\langle\delta,-t^{2} \frac{\partial \phi}{\partial x}\right\rangle \\
& =-0^{2} \cdot \frac{\partial \phi}{\partial t}(0,0)-i 0^{2} \cdot \frac{\partial \phi}{\partial x}(0,0)=0 .
\end{aligned}
$$

Ou seja, $M u \in C^{\infty}\left(\mathbb{R}^{2}\right)$ e $u \notin C^{\infty}\left(\mathbb{R}^{2}\right)$. Portanto, $M$ não é hipoelítico. 
Note que $d Z(0,0)=0$. Note também que da proposição 5.3 .9 segue que $M$ é hipoelítico em $\mathbb{R}^{2} \backslash\{0\}$.

\subsection{Estrutura Diferenciável Complexa induzida por um Campo Hipoelítico}

Seja $\Omega$ é um aberto conexo de $\mathbb{R}^{2}$ e $L$ um campo vetorial complexo não singular hipoelítico definido em $\Omega$. Vamos considerar $\Omega$ com um subespaço topológico do $\mathbb{R}^{2}$, com a topologia induzida de $\mathbb{R}^{2}$. Assim, $\Omega$ é um espaço de Hausdorff conexo e com base enumerável.

Agora, vamos aplicar os resultados anteriores do capítulo, em particular o teorema 5.3 .10 , para obter a partir de $L$ uma estrutura diferenciável complexa para $\Omega$, a qual chamaremos de estrutura diferenciável complexa induzida em $\Omega$ por $L$.

Seja $\mathcal{A}=\left\{\left(U_{\alpha}, Z_{\alpha}\right)\right\}_{\alpha \in J}$ a família dada teorema 5.2.1. Vamos mostrar que $\mathcal{A}$ é um atlas em $\Omega$. Da observação 5.2.13 temos que $Z_{\alpha}$ é uma aplicação aberta para todo $\alpha \in J$. Como $Z_{\alpha}$ é injetiva para todo $\alpha \in J$, temos que $Z_{\alpha}: U_{\alpha} \longrightarrow Z_{\alpha}\left(U_{\alpha}\right)$ é um homeomorfismo para todo $\alpha \in J$. Logo, resta mostrar a condição A.2 da definição 2.1.1 de estrutura diferenciável complexa.

Sejam $\alpha, \beta \in J$ tais que $U_{\alpha} \cap U_{\beta} \neq \emptyset$. Pelo teorema 5.3.10, existe uma função holomorfa $h_{\alpha \beta}: Z_{\beta}\left(U_{\alpha} \cap U_{\beta}\right) \longrightarrow \mathbb{C}$ tal que

$$
Z_{\beta}=h_{\alpha \beta} \circ Z_{\alpha} \text { em } U_{\alpha} \cap U_{\beta}
$$

Note que $h_{\alpha \beta} \doteq Z_{\alpha} \circ Z_{\beta}^{-1}$ em $Z_{\beta}\left(U_{\alpha} \cap U_{\beta}\right)$. Com o mesmo raciocínio, temos que $h_{\beta \alpha}=Z_{\beta} \circ Z_{\alpha}^{-1}$ em $Z_{\alpha}\left(U_{\alpha} \cap U_{\beta}\right)$, é holomorfa e é a inversa de $h_{\alpha \beta}$. Logo, $h_{\alpha \beta}$ é um biholomorfismo de $Z_{\beta}\left(U_{\alpha} \cap U_{\beta}\right)$ sobre $Z_{\alpha}\left(U_{\alpha} \cap U_{\beta}\right)$. Assim, A.2 está verificada e concluí$\operatorname{mos}$ que $\mathcal{A}=\left\{\left(U_{\alpha}, Z_{\alpha}\right)\right\}_{\alpha \in J}$ é um atlas em $\Omega$. 
Obs. 5.4.1. Note que mesmo sendo de mesma natureza que o teorema 5.3.10, o teorema 5.3.8 não é suficiente para mostrar a condição A.2 como foi feito acima usando o teorema 5.3 .10 .

Seja $\mathscr{A}=\left\{\left(U_{\alpha}, Z_{\alpha}\right)\right\}_{\alpha \in \mathcal{J}}$ a estrutura diferenciável complexa induzida pelo atlas $\mathcal{A}=$ $\left\{\left(U_{\alpha}, Z_{\alpha}\right)\right\}_{\alpha \in J}$.

Obs. 5.4.2. $S e\left(U_{\alpha}, Z_{\alpha}\right) \in \mathscr{A}$, então $L Z_{\alpha}=0$ e d $Z_{\alpha} \neq 0$ em $U_{\alpha}$.

Logo podemos denotar $\mathscr{A}$ por $\mathscr{A}_{L}$, i.e., dado um campo $L$ não singular hipoelítico em $\Omega$ podemos associar a $L$ uma estrutura diferenciável em $\Omega$, em outras palavras ficou provado o seguinte teorema:

Teorema 5.4.3. Seja L um campo vetorial complexo não singular hipoelítico definido no aberto conexo $\Omega$ de $\mathbb{R}^{2}$. Então existe uma única estrutura diferenciável complexa

$$
\mathscr{A}_{L}=\left\{\left(U_{\alpha}, Z_{\alpha}\right)\right\}_{\alpha \in \mathcal{J}}
$$

em $\Omega$ tal que

$$
L Z_{\alpha}=0 \text { em } U_{\alpha} \text { e } d Z_{\alpha} \neq 0 \text { em } U_{\alpha} \text { para todo } \alpha \in \mathcal{J} \text {. }
$$

A regularidade do campo está intimamente relacionada com a regularidade da estrutura diferenciável induzida por ele. Vamos falar um pouco sobre isso agora.

Seja $L$ um campo hipoelítico não singular definido em um aberto conexo de $\mathbb{R}^{2}$ e seja $\mathcal{A}=\left\{\left(U_{\alpha}, Z_{\alpha}\right)\right\}_{\alpha \in J}$ dado pelo teorama 5.2.1. Temos que $\mathcal{A}$ é um atlas em $\Omega$. Como foi visto na seção 2.3, o atlas $\mathcal{A}=\left\{\left(U_{\alpha}, Z_{\alpha}\right)\right\}_{\alpha \in J}$ induz um atlas real $\mathcal{A} \mathbb{R}=\left\{\left(U_{\alpha}, F_{\alpha}\right)\right\}_{\alpha \in J}$ em $\Omega$, onde $F_{\alpha}(x, t)=\left(\Re\left\{Z_{\alpha}\right\}(x, t), \Im\left\{Z_{\alpha}\right\}(x, t)\right),(x, t) \in U_{\alpha}$ para $\alpha \in J$. Seja $\mathscr{A} \mathbb{R}$ a estrutura diferenciável real induzida por $\mathcal{A} \mathbb{R}$. Assim, $\Omega$ munido da estrutura $\mathscr{A} \mathbb{R}$ é uma variedade diferenciável de dimensão 2.

Proposição 5.4.4. A identidade

$$
\begin{aligned}
I d:(\Omega, \mathscr{A} \mathbb{R}) & \longrightarrow(\Omega, i d) \\
p & \longmapsto p
\end{aligned}
$$

é diferenciável se, e somente se, L é elítico. 
Obs. 5.4.5. O par $(\Omega, i d)$ indica a variedade diferenciável real de dimenção 2 dada pelo aberto $\Omega$ com a estrutura diferenciável real induzida em $\Omega$ pelo atlas unitário $\{(\Omega, i d)\}$, onde $i d(x, t)=(x, t),(x, t) \in \Omega$.

\section{Demonstração:}

Suponha que $I d:(\Omega, \mathscr{A} \mathbb{R}) \longrightarrow(\Omega, i d)$ é diferenciável. Então, $F_{\alpha}^{-1}=i d \circ I d \circ F_{\alpha}^{-1}$ : $F_{\alpha}^{-1}\left(U_{\alpha}\right) \longrightarrow U_{\alpha}$ é um difeomorfismo, para todo $\alpha \in J$, logo, $F_{\alpha}: U_{\alpha} \longrightarrow F_{\alpha}^{-1}\left(U_{\alpha}\right)$ é um difeomorfismo, para todo $\alpha \in J$. Daí, segue que $\operatorname{grad}\left(\Re\left\{Z_{\alpha}\right\}\right), \operatorname{grad}\left(\Im\left\{Z_{\alpha}\right\}\right)$ são linearmente independentes em $U_{\alpha}$, para todo $\alpha \in J$. Pelo teorema 4.2.1, $L$ é um campo vetorial complexo elítico em $\Omega$.

Reciprocamente, se $L$ é elítico, pelo teorema 4.2.1, existe um atlas $\mathcal{B}=\left\{\left(V_{\alpha}, \zeta_{\alpha}\right)\right\}_{\alpha \in I}$ em $\Omega$, onde $\zeta_{\alpha}: V_{\alpha} \longrightarrow \mathbb{C}$ é uma função $C^{\infty}$ tal que $L \zeta_{\alpha}=0$ e $\operatorname{grad}\left(\Re\left\{\zeta_{\alpha}\right\}\right), \operatorname{grad}\left(\Im\left\{\zeta_{\alpha}\right\}\right)$ são linearmente independentes em $V_{\alpha}$ para todo $\alpha \in I$. Considere, o atlas real associado $\mathcal{B} \mathbb{R}=\left\{\left(V_{\alpha}, G_{\alpha}\right)\right\}_{\alpha \in I}$, onde $G_{\alpha}(x, t)=\left(\Re\left\{\zeta_{\alpha}\right\}(x, t), \Im\left\{\zeta_{\alpha}\right\}(x, t)\right),(x, t) \in V_{\alpha}$, para cada $\alpha \in I$. Agora, note que $\operatorname{grad}\left(\Re\left\{\zeta_{\alpha}\right\}\right), \operatorname{grad}\left(\Im\left\{\zeta_{\alpha}\right\}\right)$ são linearmente independentes em $V_{\alpha}$ para todo $\alpha \in I$, implica que $G_{\alpha}: V_{\alpha} \longrightarrow G_{\alpha}\left(V_{\alpha}\right) \subset \mathbb{R}^{2}$ é um difeomorfismo de abertos do $\mathbb{R}^{2}$ para todo $\alpha \in I$. Daí, segue que $I d:(\Omega, \mathscr{B} \mathbb{R}) \longrightarrow(\Omega, I d)$ é diferenciável, onde $\mathscr{B} \mathbb{R}$ é a estrutura diferenciável real induzida em $\Omega$ por $\mathcal{B R}$. 


\subsection{Solução do Problema}

Agora já temos feramentas suficientes para tratar do problema (*), i.e., já podemos provar o seguinte teorema:

Teorema 5.5.1. Seja L um campo vetorial complexo não singular hipoelítico definido em um aberto conexo $\Omega \subset \mathbb{R}^{2}$ onde $\pi_{1}(\Omega)=\{0\}$ ou $\pi_{1}(\Omega)=\mathbb{Z}$. Então o problema

$$
\text { (*) } \quad L Z=0, d Z \neq 0 \text { e } Z \text { injetiva em } \Omega
$$

tem solução global.

\section{Demonstração:}

Vamos usar os teoremas de uniformização de superfícies de Riemann discutidos no capítulo 2 para obter a solução do problema $(*)$. Para isso, seja $\mathscr{A}_{L}$ a estrutura diferenciável complexa definida em $\Omega$ por $L$. O conjunto $\Omega$ com a estrutura $\mathscr{A}_{L}$, que denotaremos por $\left(\Omega, \mathscr{A}_{L}\right)$, é uma superfície de Riemann.

Primeiro suponha que $\Omega$ é um aberto simplesmente conexo do $\mathbb{R}^{2}$. Assim $\left(\Omega, \mathscr{A}_{L}\right)$ é uma superfície de Riemann simplesmente conexa. Pelo Teorema 2.2.2, $\left(\Omega, \mathscr{A}_{L}\right)$ é biholomorfa a uma das seguintes superfícies de Riemann: $\overline{\mathbb{C}}, \mathbb{C}$ e $\mathbb{D}$. Como $\overline{\mathbb{C}}$ é compacta e $\Omega$ é um aberto de $\mathbb{R}^{2}$, temos que $\overline{\mathbb{C}}$ e $\left(\Omega, \mathscr{A}_{L}\right)$ não são biholomorfos. Assim $\left(\Omega, \mathscr{A}_{L}\right)$ é biholomorfa a $\mathbb{C}$ ou $\mathbb{D}$. Logo, existe uma função holomorfa e injetiva de $\left(\Omega, \mathscr{A}_{L}\right)$ em $\mathbb{C}$. Agora, suponha que $\pi_{1}(\Omega)=\mathbb{Z}$. Pelo teorema 2.2.3, $\left(\Omega, \mathscr{A}_{L}\right)$ é biholomorfa a uma das seguintes superfícies de Riemann: $\mathbb{C}^{*}, \mathbb{D}^{*}$ e $A_{r}$. Logo, existe uma função holomorfa e injetiva de $\left(\Omega, \mathscr{A}_{L}\right)$ em $\mathbb{C}$. Portanto, se $\pi_{1}(\Omega)=\{0\}$ ou $\pi_{1}(\Omega)=\mathbb{Z}$, temos que existe

$$
Z: \Omega \longrightarrow \mathbb{C}
$$

tal que

$$
h_{\alpha} \doteq Z \circ Z_{\alpha}^{-1}: Z_{\alpha}\left(U_{\alpha}\right) \longrightarrow \mathbb{C}
$$


é holomorfa injetora para toda $\alpha \in \mathcal{J}$. Mais que isso, $h_{\alpha} \doteq Z \circ Z_{\alpha}^{-1}: Z_{\alpha}\left(U_{\alpha}\right) \longrightarrow Z\left(U_{\alpha}\right)$ é um biholomorfismo. Logo, para cada $\alpha \in \mathcal{J}$, a restrição de $Z$ a $U_{\alpha}$ pode ser escrita da forma

$$
\left.Z\right|_{U_{\alpha}}=h_{\alpha} \circ Z_{\alpha} \text { em } U_{\alpha}
$$

Em particular, segue que $\left.Z\right|_{U_{\alpha}} \in C^{\infty}\left(U_{\alpha}\right)$ para todo $\alpha \in \mathcal{J}$. Como $\left\{U_{\alpha}\right\}_{\alpha \in \mathcal{J}}$ é uma cobertura aberta de $\Omega$, temos que $Z \in C^{\infty}(\Omega)$.

Dado $\alpha \in \mathcal{J}$, seja $F_{\alpha}(x, t)=\left(\Re\left\{Z_{\alpha}\right\}(x, t), \Im\left\{Z_{\alpha}\right\}(x, t)\right),(x, t) \in U_{\alpha}$ e $\tilde{h_{\alpha}}\left(x_{1}, x_{2}\right) \doteq$ $h_{\alpha}\left(x_{1}+i x_{2}\right),\left(x_{1}, x_{2}\right) \in F_{\alpha}\left(U_{\alpha}\right)=\left\{\left(x_{1}, x_{2}\right) \in \mathbb{R}^{2} ; x_{1}+i x_{2} \in Z_{\alpha}\left(U_{\alpha}\right)\right\}$. Como $h_{\alpha}$ é um biholomorfismo, temos que

$$
\frac{\partial \tilde{h_{\alpha}}}{\partial \bar{z}}\left(x_{1}, x_{2}\right)=0 \text { e } \frac{\partial \tilde{h}_{\alpha}}{\partial z}\left(x_{1}, x_{2}\right) \neq 0, \forall\left(x_{1}, x_{2}\right) \in F_{\alpha}\left(U_{\alpha}\right) .
$$

Daí, $d\left(\left.Z\right|_{U_{\alpha}}\right)=$

$$
\begin{aligned}
& =\left(\frac{\partial\left(\overline{Z_{\alpha}}\right)}{\partial x}\left(\frac{\partial \tilde{h_{\alpha}}}{\partial \bar{z}} \circ F_{\alpha}\right)+\frac{\partial\left(Z_{\alpha}\right)}{\partial x}\left(\frac{\partial \tilde{h_{\alpha}}}{\partial z} \circ F_{\alpha}\right)\right) d x+\left(\frac{\partial\left(\overline{Z_{\alpha}}\right)}{\partial t}\left(\frac{\partial \tilde{h_{\alpha}}}{\partial \bar{z}} \circ F_{\alpha}\right)+\frac{\partial\left(Z_{\alpha}\right)}{\partial t}\left(\frac{\partial \tilde{h_{\alpha}}}{\partial z} \circ F_{\alpha}\right)\right) d t \\
& =\frac{\partial\left(Z_{\alpha}\right)}{\partial x}\left(\frac{\partial \tilde{h_{\alpha}}}{\partial z} \circ F_{\alpha}\right) d x+\frac{\partial\left(Z_{\alpha}\right)}{\partial t}\left(\frac{\partial \tilde{h_{\alpha}}}{\partial z} \circ F_{\alpha}\right) d t \\
& =\left(\frac{\partial \tilde{h_{\alpha}}}{\partial z} \circ F_{\alpha}\right)\left(\frac{\partial\left(Z_{\alpha}\right)}{\partial x} d x+\frac{\partial\left(Z_{\alpha}\right)}{\partial t} d t\right) \\
& =\left(\frac{\partial \tilde{h_{\alpha}}}{\partial z} \circ F_{\alpha}\right) d Z_{\alpha} .
\end{aligned}
$$

Logo, $d\left(\left.Z\right|_{U_{\alpha}}\right)(x, t) \neq 0$, para todo $(x, t) \in U_{\alpha}$. Pela arbitrariedade de $\alpha \in \mathcal{J}$, temos $d Z \neq 0$ em $U_{\alpha}$ para todo $\alpha \in \mathcal{J}$. Como $\left\{U_{\alpha}\right\}_{\alpha \in \mathcal{J}}$ é uma cobertura aberta de $\Omega$, temos que $d Z \neq 0$ em $\Omega$.

Para cada $\alpha \in \mathcal{J}, L Z_{\alpha}=0$ em $U_{\alpha}, h_{\alpha}$ é holomorfa em $Z_{\alpha}\left(U_{\alpha}\right)$ e $Z=h_{\alpha} \circ Z_{\alpha}$ em $U_{\alpha}$. Logo,

$$
L Z(p)=L\left(h_{\alpha} \circ Z_{\alpha}\right)(p)=0, \text { para todo } p \in U_{\alpha} .
$$

Pela arbitrariedade de $\alpha \in \mathcal{J}$ e pelo fato de $\left\{U_{\alpha}\right\}_{\alpha \in \mathcal{J}}$ ser uma cobertura aberta de $\Omega$, 


$$
L Z=0 \text { em } \Omega .
$$

Portanto, $Z$ é a solução do problema.

Obs. 5.5.2. A solução $Z$ do problema (*) obtida acima é uma aplicação aberta.

Uma consequência da existência de solução do problema $(*)$ é um resultado sobre a estrutura local das soluções descrito pelo corolário abaixo.

Corolário 5.5.3. Seja L um campo vetorial complexo não singular e hipoelítico definido no aberto $\Omega$ de $\mathbb{R}^{2}$ tal que $\pi_{1}(\Omega)=\{0\}$ ou $\pi_{1}(\Omega)=\mathbb{Z}$. Então existe uma função $C^{\infty}$ injetiva $Z: \Omega \longrightarrow \mathbb{C}$ com $d Z \neq 0$ em $\Omega$, tal que $L Z=0$ em $\Omega$ e para toda $u \in \mathscr{D}^{\prime}(U)$, onde $U$ é um aberto de $\Omega$, com $L u=0$ em $U$, então

$$
u=h \circ Z \text { em } U \text {, }
$$

onde $h: Z(U) \longrightarrow \mathbb{C}$ é uma função holomorfa.

\section{Demonstração:}

Seja $\mathscr{A}_{L}$ a estrutura diferenciável complexa induzida em $\Omega$ por $L$. Seja $Z$ a solução do problema $(*)$ dada pelo teorema 5.5.1. Com uma simples verificação, temos que $Z \in \mathscr{A}_{L}$. Daí, o resultado segue aplicando o teorema 5.3.10.

Baouendi e Treves provaram um teorema de representação local para as soluções de $L u=$ 0. O que o corolário 5.5.3 diz a mais é que, no caso hipoelítico, basta usar uma única $Z$ global e toda solução $u \in C^{\infty}(U)$ (ou $\left.u \in \mathscr{D}^{\prime}(U)\right)$ de $L u=0$ será da forma $u=h \circ Z$ no próprio aberto $U$. 


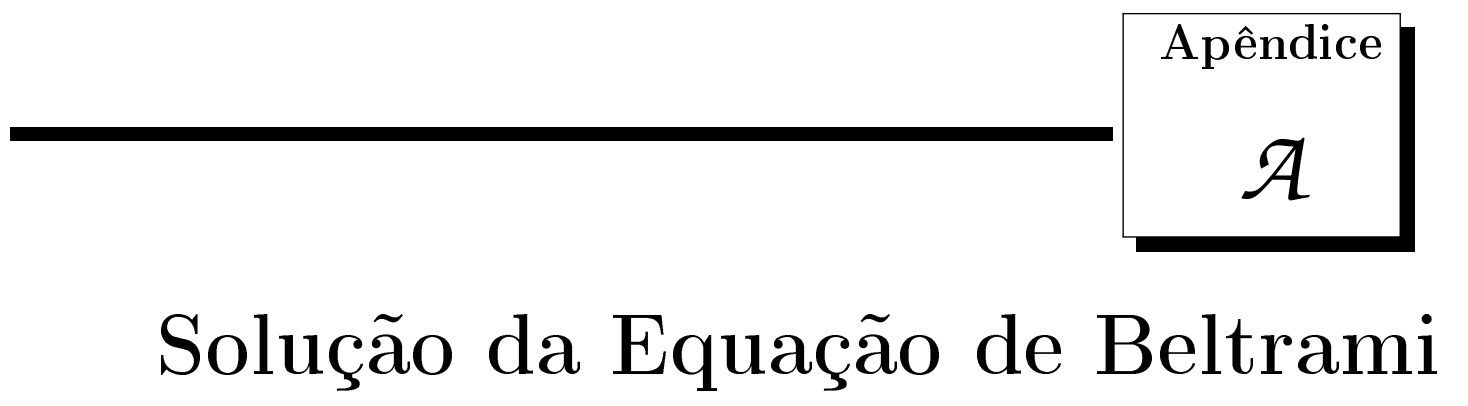

\section{A.1 Espaços de Hölder}

Definição A.1.1. Dizemos que uma função $f$ satisfaz a condição de Hölder em uma região $\Omega$, com expoente $\alpha, 0<\alpha<1$, se existe uma constante $K$ tal que

$$
|f(x)-f(y)| \leq K|x-y|^{\alpha}
$$

para todos $x, y \in \Omega$. Neste caso, $f$ também é chamada de Hölder contínua.

É claro que se $f$ é Hölder contínua em $\Omega$, então $f$ é uniformemente contínua; além disso, se $\Omega$ é limitado, então $f$ é limitada.

Denotamos por $C^{\alpha}(\Omega)$ o espaço vetorial das funções $f: \Omega \longrightarrow \mathbb{C}$ que satisfazem a condição de Hölder em $\Omega$ com expoente $\alpha$. Além disso, denotamos

$$
[f]_{(\Omega, \alpha)}=\sup _{p_{1}, p_{2} \in \Omega, p_{1} \neq p_{2}} \frac{\left|f\left(p_{1}\right)-f\left(p_{2}\right)\right|}{\left|p_{1}-p_{2}\right|^{\alpha}} .
$$

Note que $[f]_{(\Omega, \alpha)}=0$ se $f \in C^{\alpha}(\Omega)$ é constante.(Além disso, se $\Omega^{\prime} \subset \Omega$ então $[f]_{\left(\Omega^{\prime}, \alpha\right)} \leq$ $\left.[f]_{(\Omega, \alpha)}\right)$. Observe que $[\cdot]_{(\Omega, \alpha)}$ é uma seminorma em $C^{\alpha}(\Omega)$.

Segue que se $\Omega$ é limitado e $A, B>0$ constantes, então 


$$
\|f\| \doteq A \sup _{p \in \Omega}|f(p)|+B[f]_{(\Omega, \alpha)}
$$

é uma norma em $C^{\alpha}(\Omega)$. Mais ainda, nessas condições $\left(C^{\alpha}(\Omega),\|\cdot\|\right)$ é um espaço de Banach. Note que $C^{\alpha}(\Omega)$ é fechado em relação ao produto usual de funções, isto é, se $f$, $g \in C^{\alpha}(\Omega)$ então $f g \in C^{\alpha}(\Omega)$, além disso, vale

$$
\|f g\| \leq\|f\|\|g\| .
$$

\section{A.2 A Equação de Beltrami e o Método do Ponto Fixo}

Definição A.2.1. Seja $\Omega \subset \mathbb{R}^{2}$, e seja $\mu: \Omega \longrightarrow \mathbb{C}$. A equação

$$
\frac{\partial}{\partial \bar{z}}=\mu \frac{\partial}{\partial z}
$$

é chamada equação de Beltrami. A função $\mu$ é chamada coeficiente de Beltrami da equação.

Denotamos por $B_{r} \doteq\left\{p \in \mathbb{R}^{2} ;|p|<r\right\}, r>0$.

Vamos utilizar os dois lemas seguintes; a demonstração dos lemas pode ser vista em $[7]$.

Lema A.2.2. Seja $f \in C^{\alpha}\left(B_{r}\right)$. A função $h$ definida por

$$
h(z)=-\frac{1}{\pi} \iint_{B_{r}} \frac{f(\zeta)}{(\zeta-z)} d \xi d \eta, \quad \zeta=\xi+i \eta
$$

possui derivadas parciais contínuas em $B_{r}$. Além disso,

$$
\frac{\partial h}{\partial \bar{z}}(z)=f(z) \text { e } \frac{\partial h}{\partial z}(z)=-\frac{1}{\pi} \iint_{B_{r}} \frac{f(\zeta)-f(z)}{(\zeta-z)^{2}} d \xi d \eta .
$$

Lema A.2.3. Seja $f \in C^{\alpha}\left(B_{r}\right)$, e seja

$$
p(z)=-\frac{1}{\pi} \iint_{B_{r}} \frac{f(\zeta)-f(z)}{(\zeta-z)^{2}} d \xi d \eta, \quad \zeta=\xi+i \eta .
$$

então existe uma constante $C>0$ dependendo apenas de $\alpha$ tal que

$$
|p(z)| \leq C r^{\alpha}[f]_{\left(B_{r}, \alpha\right)}
$$


e p(z) satisfaz a condição de Hölder com expoente $\alpha$ e coeficiente $C[f]_{\left(B_{r}, \alpha\right)}$.

Teorema A.2.4. Se $\mu(z)$ satisfaz a condição de Hölder com expoente $\alpha$ em uma vizinhança de 0 e $|\mu(z)|<1$, então a equação de Beltrami possui solução $\omega(z)$ em uma vizinhança da origem tal que

$$
\frac{\partial \omega}{\partial z} \neq 0
$$

e as derivadas de $\omega(z)$ satisfazem a condição de Hölder com expoente $\alpha$.

Demonstração: Sem perda de generalidade podemos assumir que

$$
\mu(0)=0
$$

De fato, podemos reduzir o caso geral ao caso $\mu(0)=0$ com uma mudança de variáveis. Considere $\zeta=z+\mu(0) \bar{z}$, com $z=x+i y$. Afirmamos que $\zeta$ induz uma mudança de variáveis em $\mathbb{R}^{2}$. De fato, seja

$$
\begin{aligned}
F: & \mathbb{R}^{2} \\
(x, y) & \longmapsto(u, v)
\end{aligned}
$$

onde $u=\Re\{\zeta\}$ e $v=\Im\{\zeta\}$. Temos que $F$ é $C^{\infty}$ e com um simples cálculo vemos que $|\mu|<1$ implica $F$ bijeção e $\operatorname{det}(J(f)(x, y)) \neq 0$ para todo $(x, y) \in \mathbb{R}^{2}$. Portanto, $F$ é uma mudança global de coordenadas em $\mathbb{R}^{2}$ para $|\mu|<1$. Com as novas variáveis a equação de Beltrami torna-se

$$
\frac{\partial \omega}{\partial \bar{\zeta}}=\hat{\mu}(\zeta) \frac{\partial \omega}{\partial \zeta}
$$

onde

$$
\hat{\mu}(\zeta)=\frac{\mu(\zeta)-\mu(0)}{1-\overline{\mu(0)} \mu(\zeta)} .
$$

De fato, considere o operador $P=\frac{\partial}{\partial \bar{z}}-\mu \frac{\partial}{\partial z}$. Nas novas variáveis $P$ fica

$$
L=P \zeta \frac{\partial}{\partial \zeta}+P \bar{\zeta} \frac{\partial}{\partial \bar{\zeta}}=(\mu(0)-\mu) \frac{\partial}{\partial \zeta}+(1-\mu \overline{\mu(0)}) \frac{\partial}{\partial \bar{\zeta}}
$$

Se $\omega$ é solução da equação de Beltrami, então $\omega$ é solução de $P$, e assim 


$$
\begin{gathered}
0=(\mu(0)-\mu) \frac{\partial \omega}{\partial \zeta}+(1-\mu \overline{\mu(0)}) \frac{\partial \omega}{\partial \bar{\zeta}} \\
\Longrightarrow(\mu-\mu(0)) \frac{\partial \omega}{\partial \zeta}=(1-\mu \overline{\mu(0)}) \frac{\partial \omega}{\partial \bar{\zeta}} \\
\Longrightarrow \frac{\partial \omega}{\partial \bar{\zeta}}=\frac{(\mu-\mu(0))}{(1-\mu \overline{\mu(0)})} \frac{\partial \omega}{\partial \zeta}
\end{gathered}
$$

Note que

$$
\hat{\mu}(\zeta)=\left(\phi_{\mu(0)} \circ \mu\right)(\zeta)
$$

onde

$$
\phi_{\mu(0)}(z)=\frac{z-\mu(0)}{1-\overline{\mu(0)} z}
$$

é um biholomorfismo de $\overline{\mathbb{D}}=\{z \in \mathbb{C} ;|z| \leq 1\}$ e satisfaz $\phi_{\mu(0)}(\mu(0))=0$; assim, $\hat{\mu}(0)=0$ e como $|\mu(\zeta)|<1$ temos que $|\hat{\mu}(\zeta)|<1$. Além disso, segue que existe uma constante $K$, tal que $\left|\phi_{\mu(0)}\left(z_{1}\right)-\phi_{\mu(0)}\left(z_{2}\right)\right| \leq K\left|z_{1}-z_{2}\right|$, para todo $z_{1}, z_{2} \in \overline{\mathbb{D}}$. Daí segue que $\hat{\mu}$ satisfaz a condição de Hölder com expoente $\alpha$.

De agora adiante, vamos assumir que $\mu \in C^{\alpha}\left(B_{r}\right), r>0$, tal que $\mu(0)=0,|\mu|<1$.

A demonstração da existência da solução é uma aplicação do teorema do Ponto Fixo de Banach.

Definimos

$$
\|\omega\|_{r} \doteq \sup _{p \in B_{r}}|\omega(p)|+r^{\alpha}[\omega]_{\left(B_{r}, \alpha\right)} \text { para } \omega \in C^{\alpha}\left(B_{r}\right) .
$$

Como vimos anteriormente $\|\cdot\|_{r}$ é uma norma em $C^{\alpha}\left(B_{r}\right)$ e $\left(C^{\alpha}\left(B_{r}\right),\|\cdot\|_{r}\right)$ é um espaço de Banach.

Seja $T: C^{\alpha}\left(B_{r}\right) \longrightarrow C^{\alpha}\left(B_{r}\right)$ dado por

$$
\langle T, \omega\rangle(x, y)=-\frac{1}{\pi} \iint_{B_{r}} \frac{\mu(\zeta) \omega(\zeta)-\mu(z) \omega(z)}{(\zeta-z)^{2}} d \xi d \eta,
$$


onde $z=x+i y$ e $\zeta=\xi+i \eta$.

Pelo lema A.2.3, (fazendo $f=\mu \omega$ ) temos que $T$ está bem definido.

Note que $[\mu \omega]_{\left(B_{r}, \alpha\right)} r^{\alpha} \leq\|\mu \omega\|_{r}$. Pelo Lema A.2.3, existe uma constante $C$ que depende apenas de $\alpha$ tal que $|\langle T, \omega\rangle(p)| \leq C[\mu \omega]_{\left(B_{r}, \alpha\right)} r^{\alpha}$, para todo $p \in B_{r}$. Daí,

$$
\sup _{p \in B_{r}}|\langle T, \omega\rangle(p)| \leq C[\mu \omega]_{\left(B_{r}, \alpha\right)} r^{\alpha} \leq C\|\mu \omega\|_{r} .
$$

Novamente pelo Lema A.2.3, temos que $\langle T, \omega\rangle$ satisfaz a condição de Hölder com expoente $\alpha$, isto é, $\langle T, \omega\rangle \in C^{\alpha}\left(B_{r}\right)$, e daí

$$
r^{\alpha}[\langle T, \omega\rangle]_{\left(B_{r}, \alpha\right)} \leq C[\mu \omega]_{\left(B_{r}, \alpha\right)} r^{\alpha} \leq C\|\mu \omega\|_{r}
$$

De (A.1.1), (A.2.1) e (A.2.2) temos

$$
\|\langle T, \omega\rangle\|_{r} \leq 2 C\|\mu \omega\|_{r} \leq 2 C\|\mu\|_{r}\|\omega\|_{r}
$$

Observe que $\|\mu\|_{r}$ tende a zero quando $r \rightarrow 0$. De fato, seja $r^{\prime}<r$. Como $\mu(0)=0$ temos que $|\mu(p)| \leq[\mu]_{\left(B_{r}, \alpha\right)}|p|^{\alpha}$, para $p \in B_{r}$, logo

$$
\|\mu\|_{r^{\prime}} \leq[\mu]_{\left(B_{r}, \alpha\right)}{r^{\prime \alpha}}^{\alpha}[\mu]_{\left(B_{r^{\prime}}, \alpha\right)}{r^{\prime \alpha}}^{\prime \alpha} 2\left([\mu]_{\left(B_{r}, \alpha\right)}\right) r^{\prime \alpha}
$$

$\operatorname{logo},\|\mu\|_{r^{\prime}} \rightarrow 0$ quando $r^{\prime} \rightarrow 0$. Assim se necessário podemos reduzir $r$ de modo que

$$
2 C\|\mu\|_{r}<\frac{1}{2}
$$

De (A.2.3) e (A.2.4) temos

$$
\|\langle T, \omega\rangle\|_{r}<\frac{1}{2}\|\omega\|_{r}
$$

Agora, a partir de $T$ vamos construir uma nova aplicação em $C^{\alpha}\left(B_{r}\right)$. Definimos a aplicação $\hat{T}: C^{\alpha}\left(B_{r}\right) \longrightarrow C^{\alpha}\left(B_{r}\right)$ por

$$
\hat{T}(\omega)=\langle T, \omega\rangle+1
$$


Pelas boas propriedades de $T$ temos que $\hat{T}$ está bem definida. Além disso, $\hat{T}$ é uma contração. De fato, por (A.2.5) temos

$$
\|\hat{T}(\omega)\|_{r}=\|\langle T, \omega\rangle+1\|_{r} \leq\|\langle T, \omega\rangle\|_{r}+1<\frac{1}{2}\|\omega\|_{r}+1<2
$$

para $\omega \in C^{\alpha}\left(B_{r}\right)$ com $\|\omega\|_{r}<2$. Além disso

$$
\left\|\hat{T}\left(\omega_{1}\right)-\hat{T}\left(\omega_{2}\right)\right\|_{r}=\left\|\left\langle T, \omega_{1}-\omega_{2}\right\rangle\right\|_{r}<\frac{1}{2}\left\|\omega_{1}-\omega_{2}\right\|_{r},
$$

$\operatorname{para} \omega_{1}, \omega_{2} \in C^{\alpha}\left(B_{r}\right)$.

Pelo teorema de ponto fixo de Banach, existe um único $\omega_{0} \in C^{\alpha}\left(B_{r}\right)$ tal que $\hat{T}\left(\omega_{0}\right)=$ $\omega_{0}$. Mais precisamente, se escolhermos $\omega_{1}=0$ e pusermos $\omega_{n+1}=\hat{T}\left(\omega_{n}\right), n \in \mathbb{N}$, a sequência $\left(\omega_{n}\right)$ converge na norma $\|\cdot\|_{r}$ e $\omega_{0}=\lim \omega_{n}$.

Para completar a demonstração do teorema, vamos mostrar que a função

$$
w(z)=-\frac{1}{\pi} \iint_{B_{r}} \frac{\mu(\zeta) \omega_{0}(\zeta)}{(\zeta-z)} d \xi d \eta+z
$$

é a solução desejada.

Pelo lema (A.2.3), temos

$$
\frac{\partial w}{\partial \bar{z}}(z)=\mu(z) \omega_{0}(z)
$$

Por outro lado,

$\omega_{0}(z)=\hat{T}\left(\omega_{0}\right)(z)=\left\langle T, \omega_{0}\right\rangle(z)+1=-\frac{1}{\pi} \iint_{B_{r}} \frac{\mu(\zeta) \omega_{0}(\zeta)-\mu(z) \omega_{0}(z)}{(\zeta-z)^{2}} d \xi d \eta+1=\frac{\partial w}{\partial z}(z)$

Assim,

$$
\frac{\partial w}{\partial \bar{z}}=\mu \frac{\partial w}{\partial z}
$$

Resta mostrar que

$$
\frac{\partial w}{\partial z}(p) \neq 0 \text { para todo } p \in B_{r} \text {. }
$$


Basta verificar que $\omega_{0}(p) \neq 0$ para todo $p \in B_{r}$.

Por (A.2.5),

$$
\left|\omega_{0}(p)-1\right|=\left|\hat{T}\left(\omega_{0}\right)(p)-1\right|=\left|\left\langle T, \omega_{0}\right\rangle(p)\right| \leq\left\|T\left(\omega_{0}\right)\right\|_{r}<\frac{1}{2}\left\|\omega_{0}\right\|_{r},
$$

para todo $z \in B_{r}$. Logo basta mostrar que $\left\|\omega_{0}\right\|_{r}<2$. Observe que por (A.2.5)

$$
\|\hat{T}(\omega)\|_{r}=\|\langle T, \omega\rangle+1\|_{r} \leq\|\langle T, \omega\rangle\|_{r}+1<\frac{1}{2}\|\omega\|_{r}+1<2
$$

para $\omega \in C^{\alpha}\left(B_{r}\right)$ com $\|\omega\|_{r}<2$. Ou seja,

$$
\text { se }\|\omega\|_{r}<2 \text {, então }\|\hat{T}(\omega)\|_{r}<2 \text {. }
$$

Como $\left\|\omega_{1}\right\|<2$, por (A.2.6) temos que $\left\|\hat{T}\left(\omega_{n}\right)\right\|_{r}<2$ para todo $n \in \mathbb{N}$ e passando ao limite temos $\left\|\omega_{0}\right\| \leq 2$. Suponha que vale a igualdade por (A.2.5) temos

$$
2=\left\|\omega_{0}\right\|_{r}=\left\|\hat{T}\left(\omega_{0}\right)\right\|_{r} \leq\left\|\left\langle T, \omega_{0}\right\rangle\right\|_{r}+1<\frac{1}{2}\left\|\omega_{0}\right\|_{r}+1=2 .
$$

Absurdo. Portanto w é a solução desejada.

Obs. A.2.5. Considere o campo

$$
M=\frac{\partial}{\partial \bar{z}}-\mu \frac{\partial}{\partial z}
$$

em $\Omega \subset \mathbb{R}^{2}$ aberto, onde $\mu \in C^{\infty}(\Omega)$ é tal que $|\mu|<1$ em $\Omega$.

Afirmamos que $M$ é elítico em $\Omega$. De fato, temos

$$
M=\frac{1}{2}\left(\frac{\partial}{\partial x}+i \frac{\partial}{\partial t}\right)-\mu \frac{1}{2}\left(\frac{\partial}{\partial x}-i \frac{\partial}{\partial t}\right)=\frac{1}{2}\left[(1-\mu) \frac{\partial}{\partial x}+i(1+\mu) \frac{\partial}{\partial t}\right]
$$

em $\Omega$. Como $|\mu|<1$ em $\Omega$, temos que $1-\mu \neq 0$ em $\Omega$. Assim podemos escrever

$$
M=\frac{1}{2}(1-\mu)\left[\frac{\partial}{\partial x}+i\left(\frac{1+\mu}{1-\mu}\right) \frac{\partial}{\partial t}\right]
$$

em $\Omega$. Agora, observe que 


$$
\frac{1+\mu}{1-\mu}=\frac{(1+\mu)(1-\bar{\mu})}{(1-\mu)(1-\bar{\mu})}=\frac{(1+\mu)(1-\bar{\mu})}{|1-\mu|^{2}}=\frac{1-|\mu|^{2}+2 i \Im\{\mu\}}{|1-\mu|^{2}} .
$$

Daí,

$$
\Im\left\{i\left(\frac{1+\mu}{1-\mu}\right)\right\}=\frac{1-|\mu|^{2}}{|1-\mu|^{2}}>0 \text { em } \Omega .
$$

Daí, é fácil ver que $M$ é elítico em $\Omega$. Em particular, $M$ é hipoelítico. Assim, se $Z \in$ $\mathscr{D}^{\prime}(U), U \subset \Omega$ aberto, com

$$
\frac{\partial Z}{\partial \bar{z}}=\mu \frac{\partial Z}{\partial z}
$$

ent $\tilde{a} o \quad Z \in C^{\infty}(U)$.

Corolário A.2.6. Seja $\mu \in C^{\infty}(\Omega)$, onde $\Omega$ é um aberto de $\mathbb{R}^{2}$, com $|\mu|<1$ em $\Omega$. Então para cada $p_{0} \in \Omega$, existe uma função $C^{\infty} Z: U \longrightarrow \mathbb{C}$, onde $U$ é uma vizinhança aberta de $p_{0}$ em $\Omega$, tal que

$$
\frac{\partial Z}{\partial \bar{z}}=\mu \frac{\partial Z}{\partial z} \quad e \quad \frac{\partial Z}{\partial z} \neq 0 \text { em } U
$$

\section{Demonstração:}

Seja $p_{0} \in \Omega$. Considere

$$
T(p)=p-p_{0}, p \in \Omega
$$

Temos que $T$ é um difeomorfismo $C^{\infty}$ de $\Omega$ sobre $T(\Omega)$. Assim,

$$
\begin{aligned}
M_{T} & =\left(M\left(T_{1}\right) \circ T^{-1}\right) \frac{\partial}{\partial y_{1}}+\left(M\left(T_{2}\right) \circ T^{-1}\right) \frac{\partial}{\partial y_{2}} \\
& =(1-\mu) \circ T^{-1} \frac{\partial}{\partial y_{1}}+i(1+\mu) \circ T^{-1} \frac{\partial}{\partial y_{2}} \\
& =\frac{\partial}{\partial \bar{z}}-\left(\mu \circ T^{-1}\right) \frac{\partial}{\partial z} \\
& =\frac{\partial}{\partial \bar{z}}-\left(T^{-1}\right)^{*}(\mu) \frac{\partial}{\partial z} .
\end{aligned}
$$

Note que $\left(T^{-1}\right)^{*}(\mu) \in C^{\infty}(T(\Omega))$ e $\left|\left(T^{-1}\right)^{*}(\mu)\right|<1$ em $T(\Omega)$. Seja $r>0$ tal que $\bar{D}_{r}(0) \subset$ $T(\Omega)$, onde $D_{r}(0)=\left\{p \in \mathbb{R}^{2} ;|p|<r\right\}$. Segue do teorema do valor médio que existe uma constante $K>0$ tal que 


$$
\left|\left(T^{-1}\right)^{*}(\mu)(p)-\left(T^{-1}\right)^{*}(\mu)(q)\right| \leq K|p-q|
$$

para todo $p, q \in D_{r}(0)$. Sem perda, podemos supor que $r<1 / 2$. Logo, $|p-q|<1$ para todo $p, q \in D_{r}(0)$. Daí, se $0<\alpha<1$, então $|p-q|<|p-q|^{\alpha}$, para todo $p, q \in D_{r}(0)$. Assim,

$$
\left|\left(T^{-1}\right)^{*}(\mu)(p)-\left(T^{-1}\right)^{*}(\mu)(q)\right| \leq K|p-q|^{\alpha}
$$

para todo $p, q \in D_{r}(0)$. Portanto, $\left(T^{-1}\right)^{*}(\mu) \in C^{\alpha}\left(D_{r}(0)\right)$. Pelo teorema A.2.4 existe $w: V \longrightarrow \mathbb{C}$, onde $V$ é uma vizinhança aberta da origem, tal que $M_{T}(w)=0$ em $V$. Segue que $Z \doteq T^{*}(w) \in C^{\infty}\left(T^{-1}(V)\right)$, onde $U \doteq T^{-1}(V)$, satisfaz $M Z=0$ em $U$, e $\partial_{z} Z \neq 0$ em $U$. Note que $U$ é uma vizinhança aberta de $p_{0}$ em $\Omega$. Pela arbitrariedade de $p_{0} \in \Omega$, o corolário está demonstrado. 


\section{Referências Bibliográficas}

[1] Baouendi, M. S. e Treves, F., A local constancy principle for the solutions of certain overdetermined systems of first-order linear partial differential equations. Mathematical analysis applications, part A, Advances in mathematics supplementary studies, vol. 7A. Academic Press, 1981.

[2] Beals, R. e Fefferman, C., On local Solvability of linear partial differential equations. Ann. of Math. 97, 482-498, 1973.

[3] Bergamasco, A., Cordaro, P. e Hounie, J., Global Properties of a Class of Vector Fields in the Plane. Journal of differential equations 74, 179-199, 1988.

[4] Bergamasco, A., Resolubilidade local e hipoeliticidade de operadores diferenciais parciais lineares de primeira ordem. Dissertação de Mestrado, ICMC-USP, São Carlos, 1974 .

[5] Berhanu, S., Cordaro, P. D., Hounie, J., An Introduction to Involutive Structures. Cambridge University Press, New York, 2008.

[6] Conway, J. B., Functions of one complex variable. Springer-Verlag, New York, 1978.

[7] Courant, R. e Hilbert L., Methods of mathematical physics, VOL II Partial differential equations. Wiley classics library, Wiley-VCH Verlag GmbH \& Co. KGaA, 1962.

[8] Farkas, H. M. e Kra, I.,Riemann Surfaces. Springer-Verlag, New York, 1980. 
[9] Folland, G. B., Introduction to partial differential equations. Princeton University Press and University of Tokio Press. Princeton, New Jersey, 1976.

[10] Forster, O., Lectures on Riemann surfaces. Springer-Verlag, New York, 1981.

[11] Friedlander, F. G. e Joshi, M.,Introduction to the theory of distributions. Cambridge University Press, 1982.

[12] Hörmander, L., Propagation of singularities and semiglobal existence theorems for (pseudo-)differential operators of principal type. Ann. of Math. 108, 569 - 609, 1978.

[13] Hörmander, L., The analysis of linear partial differential operators. ( vol.1 e vol.4), Springer-Verlag, 1983.

[14] Hounie, J., Teoria elementer das distribuições. $12^{\circ}$ Colóquio brasileiro de matemática, Poços de Caldas, 1979.

[15] Kannai, Y., An unsolvable hypoelliptic differential operator. Israel J. Math. 9, 306$315,1970$.

[16] Lewy, H., An example of a smooth linear partial differential equation without solution. Ann. of Math. 66, 155-158, 1957.

[17] Lima, E. L., Análise Real, vol.1. Funções de uma variável real. Rio de Janeiro, IMPA, Coleção Matemática Universitária, 2008.

[18] Lima, E. L., Curso de análise vol. 2. Rio de Janeiro, IMPA, Projeto Euclides, 2008.

[19] Lima, E. L., Elementos de topologia geral. Rio de Janeiro, SBM, Textos Universitários, 2009.

[20] Lima, E. L., Espaços métricos. Rio de Janeiro, IMPA, Projeto Euclides, 2009.

[21] Lima, E. L., Variedades diferenciáveis. Rio de Janeiro, IMPA, Publicações Matemáticas, 2010.

[22] Lins Neto, A., Funções de uma variável complexa. Rio de Janeiro, IMPA, Projeto Euclides, 2008. 
[23] Munkres, James R., Topology. Prentice Hall. 2000.

[24] L. Nirenberg, Lectures on linear partial differential equations. Amer. Math. Sot. Region Conf. Series in Math. 17, 1973.

[25] Reed, M. e Simon, B., Methods of modern mathematical physics. Vol I: Functional analysis. Academic Press, New York and London, 1972.

[26] Da Silva, N., Unicidade no problema de cauchy para operadores de primeira ordem. Dissertação de Mestrado, ICMC-USP, São Carlos, 1982.

[27] Treves, F., Concatenations of second-order evolution equations applied to local solvability and hypoellipcity, Communications on Pure and Applied Mathematics 26, 201-250. 1973.

[28] Treves, F., Hypo-analytic structures. Local theory. Princeton University Press, 1992.

[29] Treves, F., Hypoelliptic partial differential equations of principal type. Sufficient conditions and necessary conditions*, Comm. Pure Appl. Math. 24 631-670, 1971.

[30] Treves, F., Lectures on linear partial differential equations with constant coefficient. Notas de Matemática IMPA. 27, 1961.

[31] Treves, F., Remarks about certain first-order linear PDE in two variables. Comm. PDE 5, 381-425, 1980.

[32] Treves, F., Topological vector spaces, distributions and kernels. Academic Press, New York, 1967. 